\title{
A comprehensive anatomical and phylogenetic evaluation of Dilophosaurus wetherilli (Dinosauria, Theropoda) with descriptions of new specimens from the Kayenta Formation of northern Arizona
}

\author{
Adam D. Marsh ${ }^{1,2}$ (1) and Timothy B. Rowe ${ }^{1}$ \\ ${ }^{1}$ Jackson School of Geosciences, the University of Texas at Austin, 2305 Speedway Stop C1160, Austin, Texas 78712, USA \\ <admarsh@utexas.edu><rowe@mail.utexas.edu> \\ ${ }^{2}$ Division of Resource Management, Petrified Forest National Park, 1 Park Road \#2217, Petrified Forest, Arizona 86028, USA
}

\begin{abstract}
Dilophosaurus wetherilli was the largest animal known to have lived on land in North America during the Early Jurassic. Despite its charismatic presence in pop culture and dinosaurian phylogenetic analyses, major aspects of the skeletal anatomy, taxonomy, ontogeny, and evolutionary relationships of this dinosaur remain unknown. Skeletons of this species were collected from the middle and lower part of the Kayenta Formation in the Navajo Nation in northern Arizona. Redescription of the holotype, referred, and previously undescribed specimens of Dilophosaurus wetherilli supports the existence of a single species of crested, large-bodied theropod in the Kayenta Formation. The parasagittal nasolacrimal crests are uniquely constructed by a small ridge on the nasal process of the premaxilla, dorsoventrally expanded nasal, and tall lacrimal that includes a posterior process behind the eye. The cervical vertebrae exhibit serial variation within the posterior centrodiapophyseal lamina, which bifurcates and reunites down the neck. Iterative specimen-based phylogenetic analyses result in each of the additional specimens recovered as the sister taxon to the holotype. When all five specimens are included in an analysis, they form a monophyletic clade that supports the monotypy of the genus. Dilophosaurus wetherilli is not recovered as a ceratosaur or coelophysoid, but is instead a non-averostran neotheropod in a grade with other stem-averostrans such as Cryolophosaurus ellioti and Zupaysaurus rougieri. We did not recover a monophyletic 'Dilophosauridae.' Instead of being apomorphic for a small clade of early theropods, it is more likely that elaboration of the nasals and lacrimals of stem-averostrans is plesiomorphically present in early ceratosaurs and tetanurans that share those features. Many characters of the axial skeleton of Dilophosaurus wetherilli are derived compared to Late Triassic theropods and may be associated with macropredation and an increase in body size in Theropoda across the Triassic-Jurassic boundary.
\end{abstract}

\section{Introduction}

The summer of 1942 began with Sam Welles assisting Charles Camp of the University of California Museum of Paleontology in his annual field season in the American Southwest. Camp was primarily interested in the Permian rocks near Monument Valley, and Welles spent most of the summer in the Moenkopi Formation near Cameron, Arizona. Camp returned to Berkeley, California and left Welles to meet with a trader named Richard Kerley at Tuba City, Arizona. Kerley introduced Welles to a Navajo man named Jesse Williams who had discovered the remains of a large dinosaur in 1940 near the now-famous dinosaur trackway site at Moenave, Arizona (Field Notes Summer of 1942 May 12-July 19 Arizona, The Samuel P. Welles Papers, Series 5: Field Notes, Box 10-11, University of California Museum of Paleontology Archives). With the help of Bill Rush and Ed Cott, Sam Welles opened the quarry of the holotype and paratype specimens of Dilophosaurus wetherilli (Welles, 1954, 1970) within the Kayenta Formation. Welles collected a larger individual in 1964 near the holotype locality (Field Notes 1964-1968, The Samuel P. Welles Papers, Series
5: Field Notes, Box 10-11, University of California Museum of Paleontology Archives; Welles, 1984) and later teams from the University of Texas at Austin collected two individuals near Gold Spring, AZ, 30 miles southeast from the type quarry (Rowe et al., 2011; Marsh and Rowe, 2018). Thanks to nearly 80 years of research, Dilophosaurus wetherilli has captured the interest of the public and paleontologists alike who are drawn to its silver screen stardom, its characteristic cranial anatomy, and its ecological role as the earliest large-bodied theropod dinosaur in North America.

At the time of collection of the holotype specimen of Dilophosaurus wetherilli, the Kayenta Formation was hypothesized to be Early Jurassic or Late Triassic in age, based predominantly on vertebrate biostratigraphy; 'carnosaurian' theropods and tritylodontid cynodonts had been recovered from the unit and were thought to originate in the earliest Jurassic Period and latest Triassic Period, respectively (Welles, 1954, 1984; Lewis et al., 1961; Kermack, 1982). Because it was collected a few meters above the base of the Kayenta Formation, the holotype specimen of Dilophosaurus wetherilli was considered an important biostratigraphic indicator. More recent discoveries 
of Dilophosaurus from the 'middle third' of the Kayenta Formation (Sues et al., 1994; Rowe et al., 2011) yielded radiometric dates that confirm an Early Jurassic age for some or all of the formation (see below; Marsh et al., 2014). This confirmation is significant because it means that Dilophosaurus wetherilli, Syntarsus kayentakatae Rowe, 1989, Sarahsaurus aurifontanalis Rowe et al., 2011, and Scutellosaurus lawleri Colbert, 1981 in the Kayenta Formation of western North America are separated in time by as many as $30 \mathrm{Myr}$ from non-dinosaur dinosauromorphs and small-bodied theropods, such as Coelophysis bauri Cope, 1887 (Colbert, 1989), Tawa hallae Nesbitt et al., 2009b, and Chindesaurus bryansmalli Long and Murry, 1995, which were living in the Late Triassic (Irmis et al., 2007).

Hypotheses of the evolutionary relationships of Dilophosaurus wetherilli have been in flux since Welles first attributed the taxon to Jurassic theropod groups like 'megalosaurs,' 'coelurids,' and 'carnosaurs' based on ratios of limb measurements (von Huene, 1932; Ostrom, 1978), and citing anatomical features of the taxon that are "well developed and represent a stage considerably more advanced than in the known late Triassic theropods" (Welles, 1954, p. 597; 1970, 1984). Dilophosaurus wetherilli has also been included within Coelophysidae (Paul, 1988), Dilophosauridae (Madsen and Welles, 2000; Yates, 2005), and stem-tetanuran theropods (Forster, 1999; Fig. 2.1). Several studies supported Dilophosaurus wetherilli as a member of Coelophysoidea (Holtz, 1994), which were at the time considered to be the earliest theropods within the larger group Ceratosauria (Fig. 2.2). This hypothesis was also supported in subsequent phylogenetic analyses (Rowe and Gauthier, 1990; Tykoski, 1998, 2005; Holtz, 2000; Tykoski and Rowe, 2004), or as a coelophysoid down-tree from Ceratosauria (Ezcurra and Novas, 2007; Carrano et al., 2012; Xing, 2012). The most recent consensus is that Dilophosaurus is not a member of Coelophysoidea (as defined by Nopsca, 1928; sensu Holtz, 1994) but is pulled crown-ward, so that Dilophosaurus wetherilli and coelophysoids are non-averostran neotheropods (Averostra includes the Ceratosauria and Tetanurae lineages; Carrano et al., 2002; Paul, 2002; Rauhut, 2003; Yates, 2005; Ezcurra and Cuny, 2007; Smith et al., 2007; Hendrickx and Mateus, 2014; Langer et al., 2014; You et al., 2014; Nesbitt and Ezcurra, 2015; Novas et al., 2015; Martill et al., 2016; Ezcurra, 2017; Langer et al., 2017; Zahner and Brinkmann, 2019; Fig. 2.3) along with taxa such as Zupaysaurus rougieri Arcucci and Coria, 2003 (Ezcura and Novas, 2007), Dracovenator regenti Yates, 2005, Cryolophosaurus ellioti Hammer and Hickerson, 1994 (Smith et al., 2007), Tachiraptor admirabilis Langer et al., 2014, and, depending on the phylogeny, Sinosaurus triassicus Young, 1940 (Xing, 2012) and Berberosaurus liassicus Allain et al., 2007 (Xu et al., 2009).

In addition to phylogenetic ambiguity, there remains uncertainty in the alpha taxonomy of Dilophosaurus wetherilli. While most studies treat the genus as monotypic (or at least only score the hypodigm in analyses), Welles expressed hesitation that the large specimen he collected in 1964 was even the same genus as $D$. wetherilli, and it was for that reason that he refrained from describing the specimen, stating "differences in the skull proportions, vertebrae, and especially the femur have forced the conclusion that there are indeed two distinct genera" (Welles, 1984, p. 89). More recently, variation in the anatomy of the interdental plates has been cited to support multiple taxa of crested theropods in the Kayenta Formation (Hendrickx and Mateus, 2014). Of course, many of these differences could be owing to intraspecific variation (Carrano et al., 2012), ontogenetic variation (Tykoski, 2005; Griffin, 2018), sexual dimorphism (Gay, 2005), or diagenetic alteration; and taxonomic hypotheses must be tested in a phylogenetic framework. Further, a Dilophosaurus-like animal has been hypothesized to be the trackmaker of the ichnotaxa Dilophosauripus williamsi Welles, 1971, Kayentapus hopii Welles, 1971, and Eubrontes giganteus Hitchcock, 1845 in the Glen Canyon Group of the American Southwest (Lucas et al., 2006a, 2006b; Milner et al., 2006, 2009; Buckley et al., 2015), not to mention similar trace fossils found in Triassic- and Jurassic-aged rocks in the eastern United States, Europe, Africa, and China (Hitchcock, 1845; Lull, 1904; Haubold, 1986; Olsen et al., 1997; Lockley et al., 2011; Xing et al., 2016, 2017; Sciscio et al., 2017). The biostratigraphic significance of these ichnotaxa is more difficult to assess without phylogenetic or taxonomic control based on the skeletal anatomy of Dilophosaurus wetherilli.

Given its phylogenetic position either as the largest coelophysoid or stem-averostran neotheropod, Dilophosaurus is important in reconstructing the early history of Theropoda and in establishing the suite of character states found in Averostra and later in Aves. Here, we present a comprehensive description and fully illustrate all of the referred specimens of Dilophosaurus wetherilli, including Welles' large 1964 specimen and those at the University of Texas at Austin, in order to elucidate shared and derived character states. We score all of these individuals as terminal taxa in an updated phylogenetic analysis to assess the alpha taxonomy of Dilophosaurus, to hypothesize the evolutionary relationships of early theropod dinosaurs, and to better understand the anatomical changes that occurred in the Early Jurassic that helped theropods become the most disparate and diverse group of vertebrates on land.

\section{Methods}

Collection, preparation, and photography.-Preparation of the hypodigm of Dilophosaurus wetherilli was completed by Wann Langston, Jr., then a graduate student under Charles Camp's supervision at the University of California, Berkeley although most of the cranial material and limbs of the holotype and paratype had already been prepared by Martin Calkin by the time Langston arrived in Berkeley in 1948 (Wann Langston undated, Wann Langston Papers, Series III: Museum Notes 1968-2005, Box VPA001/26, Texas Vertebrate Paleontology Archives). Langston supervised a student, Philip Goerl, in completing the preparation of the rest of the hypodigm, including the holotype trunk, manus, and pedes. According to Langston, the in-situ opisthotonic position of the skeleton was verbally communicated by Welles (Wann Langston undated, Wann Langston Papers, Series III: Museum Notes 1968-2005, Box VPA001/26, Texas Vertebrate Paleontology Archives). Owing to the fragmentary nature of the skeleton, Langston used plaster to reconstruct missing anatomy based on Allosaurus and Streptospondylus (Wann Langston undated, Wann Langston Papers, Series III: Museum Notes 1968-2005, Box VPA001/26, Texas Vertebrate Paleontology Archives). Langston also supervised the completion of the panel mount of the holotype 


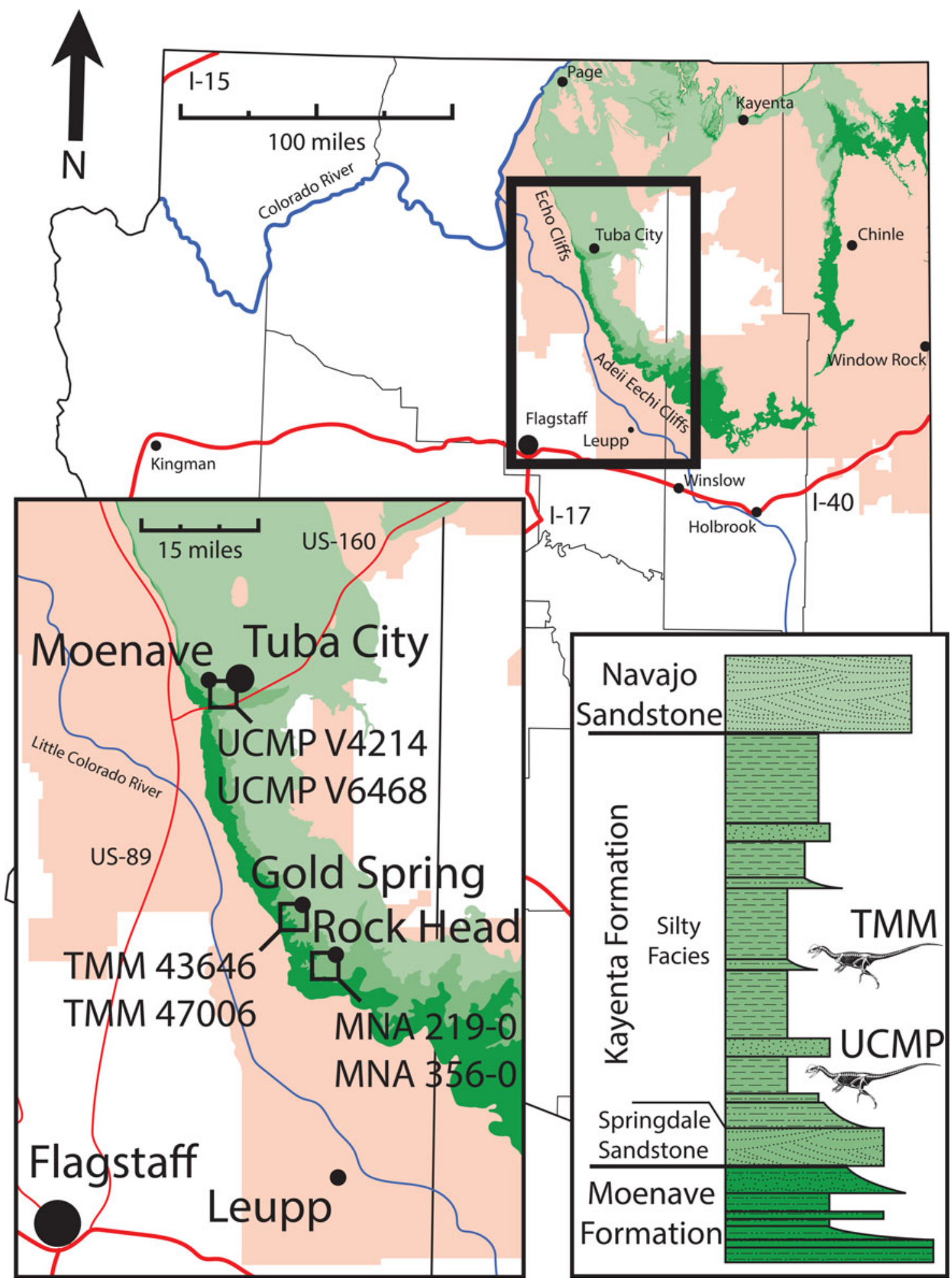

Figure 1. Localities from which Dilophosaurus wetherilli (Welles, 1954) has been collected in northern Arizona. The shaded region in the northeastern corner of the state represents the Navajo Nation. The inset stratigraphic column idealizes the section near Tuba City and Gold Spring, AZ. The dark green unit underlying the Kayenta Formation represents the Moenave Formation and the Wingate Sandstone in the western and eastern half of the Navajo Nation, respectively. Outcrop area modified from Cooley et al. (1969).

bones that is figured in the original description of the taxon (Wann Langston undated, Wann Langston Papers, Series III: Museum Notes 1968-2005, Box VPA001/26, Texas Vertebrate Paleontology Archives; Welles, 1954, fig. 1; Bell et al., 2013).
The holotype and paratype specimens were collected using plaster and burlap field jackets and paper packaging (Field Notes Summer of 1942 May 12-July 19 Arizona, The Samuel P. Welles Papers, Series 5: Field Notes, Box10-11, University 


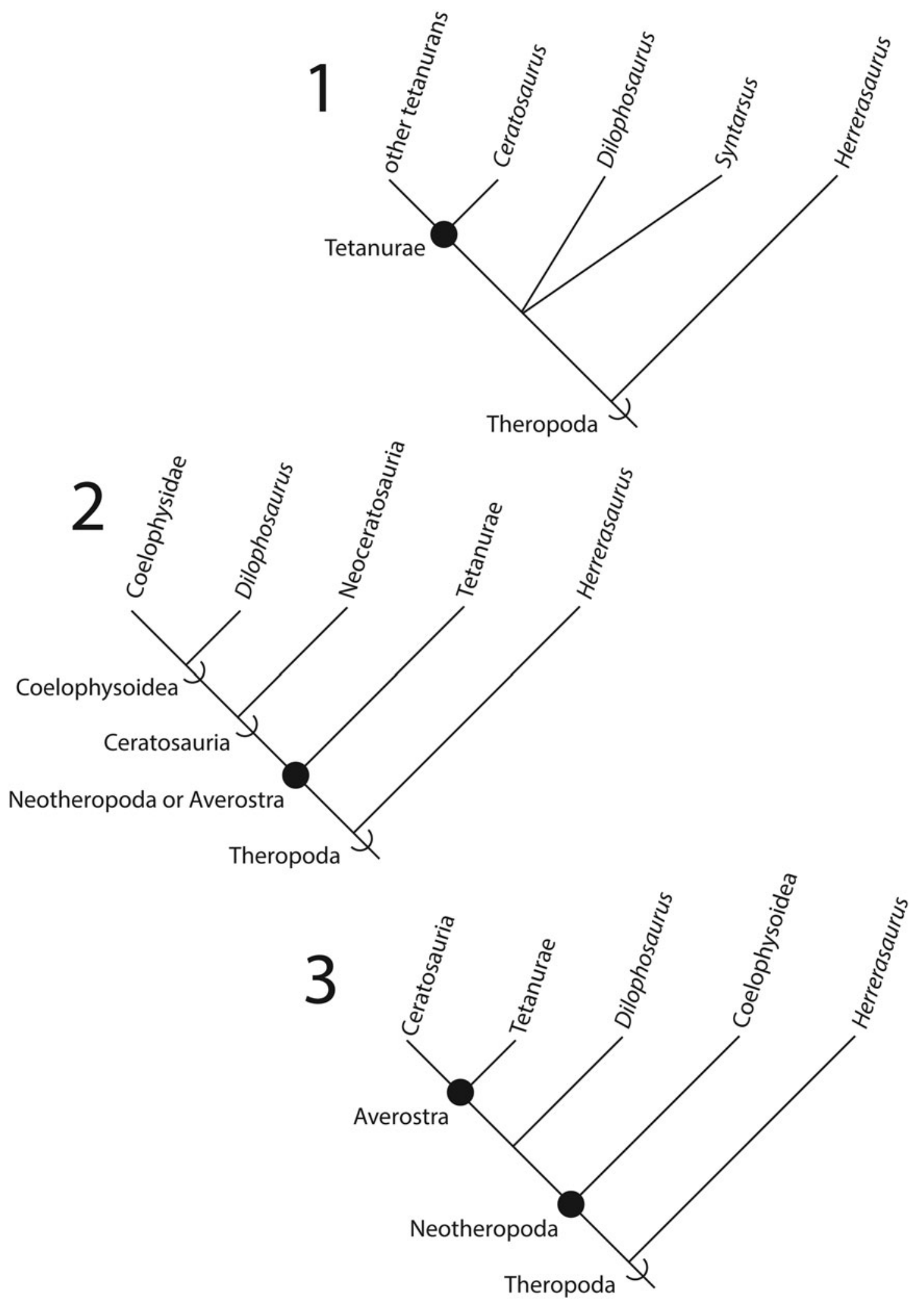

Figure 2. The phylogentic position of Dilophosaurus wetherilli in previous hypotheses using parsimony methods: (1) as a non-tetanuran theropod (Forster, 1999), (2) as a coelophysoid ceratosaur (e.g., Rowe and Gauthier, 1990; Holtz, 1994, 2000; Tykoski, 1998, 2005; Tykoski and Rowe, 2004; Ezcurra, 2006; Ezcurra and Cuny, 2007; Ezcurra and Novas, 2007; Carrano et al., 2012; Xing, 2012; Novas et al., 2015), and (3) as a stem-averostran neotheropod (e.g., Carrano et al., 2002; Rauhut, 2003; Yates, 2005; Smith et al., 2007; Hendrickx and Mateus, 2014; Langer et al., 2014; You et al., 2014; Nesbitt and Ezcurra, 2015; Martill et al., 2016; Ezcurra, 2017; Langer et al., 2017; Zahner and Brinkmann, 2019). The highlighted node in (2) represents either the most recent common ancestor of all neotheropods or averostrans, depending on the analysis. 
of California Museum of Paleontology Archives; Wann Langston undated, Wann Langston Papers, Series III: Museum Notes 1968-2005, Box VPA001/26, Texas Vertebrate Paleontology Archives). Most of the hypodigm was prepared using a 'needle' (most likely a steel phonograph needle in a pin vise), but the consolidants and adhesives used are unknown. Several iterations of restoration were performed on the vertebral column following initial preparation, construction of a wall mount, and later changes made after the discovery of UCMP 77270. A yellowed plaster represents what is assumed to be Wann Langston's original restoration. Unpolished white plaster was added later by Welles. The frame of the panel mount was built from $2 \times 4$ lumber with a backing of hardware cloth and layers of plaster and burlap (Wann Langston undated, Wann Langston Papers, Series III: Museum Notes 1968-2005, Box VPA001/26, Texas Vertebrate Paleontology Archives). The bones of UCMP 37302 were arranged within the frame and bolted to additional $2 \times 4 \mathrm{~s}$ when necessary before the frame was filled with plaster and occasional burlap strips for added strength. The outer layer of plaster was then sculpted with 'vibrotools' to texture the smooth surface (Wann Langston undated, Wann Langston Papers, Series III: Museum Notes 1968-2005, Box VPA001/26, Texas Vertebrate Paleontology Archives).

Langston had left the UCMP by the time Welles found UCMP 77270 in 1964, so Welles and Robert Long removed the holotype bones from the plaster wall mount in order to reconstruct it based on the new specimen (Letter to Wann Langston July 1 1977, The Samuel P. Welles Papers, Series 1: Correspondence 1936-1996, Box 4, University of California Museum of Paleontology Archives; The Tuba City Dinosaur, The Samuel P. Welles Papers, Series 2: Dinosaur Files, University of California Museum of Paleontology Archives). The skull of UCMP 77270 is very fragile and is housed in two clamshell plaster cradles. A plastic support rod connects the jugal process of the maxilla and the postorbital process of the lacrimal. Flat plastic strips have been added to the inside of the antorbital fenestra for additional support and to prevent researchers from picking the specimen up in that region. The dorsal region of the skull was soaked in epoxy, presumably to increase the stability of the crest, but in places the thick layer of epoxy obscures anatomy.

Partially sculpted mounts and casts of the UCMP material of Dilophosaurus wetherilli can be found at the American Museum of Natural History (on exhibit) in New York City, NY, the Navajo Nation Museum (in the director's office) in Window Rock, AZ, the Museum of Northern Arizona (on exhibit and in the preparation lab) in Flagstaff, AZ, the University of California Museum of Paleontology (in collections and storage) in Berkeley, CA, the Community College of San Francisco (on exhibit, formerly at the California Academy of Sciences) in San Francisco, CA, the Science Museum of Minnesota (on exhibit) in St. Paul, MN, the Royal Ontario Museum (temporarily off exhibit) in Toronto, Ontario, the Royal Tyrrell Museum (on exhibit) in Drumheller, Alberta, and the Australian Museum (on exhibit) in Sydney, Australia. Fleshed-out sculptures of Dilophosaurus wetherilli are on exhibit at the Dinosaur Discovery Center at Johnson Farm in St. George, UT, the Arizona Museum of Natural History in Mesa, AZ, and at Dinosaur State Park in Rocky Hill, CT.
The preparation of TMM 43646-1 and TMM 47006-1 was accomplished using carbide needles, pneumatic air scribes, and Paraloid B-72 consolidant. TMM 43646-1 was found in the same quarry as the two individuals that form the hypodigm of Sarahsaurus aurifontanalis (Rowe et al., 2011), and individual elements were numbered prior to each animal receiving a unique number. The bones of Dilophosaurus wetherilli from that quarry are numbered TMM 43646-1.x (where $\mathrm{x}$ is the original element number assigned during preparation), and those of the holotype and paratype of Sarahsaurus aurifontanalis are numbered TMM 43646-2.x and TMM 43646-3.x, respectively.

Photographs were taken using a Nikon D70S (UCMP specimens), Sony NEX-5R (TMM specimens), and Canon PowerShot ELPH 130 IS (MNA specimens) tethered to a MacBook Pro running Sofortbild (version 1.5), when possible. Photo stacking was accomplished using HeliconFocus Lite (version 6). Linear measurements $200 \mathrm{~mm}$ and less were measured using Pittsburgh electronic digital calipers and those longer than $200 \mathrm{~mm}$ were measured using a paper tape measure. All linear measurements and digital photographs for the UCMP and TMM specimens can be found in the Supplemental Data.

Computed tomography. - The braincase of TMM 47006-1 was scanned at the University of Texas High-Resolution X-ray CT Facility using a North Star Imaging scanner that comprises a Fein Focus High Power $200 \mathrm{kV}$ source $(0.24 \mathrm{~mA})$, aluminum filter, and Perkin Elmer detector. The voxel size is $50.5 \mu$, resulting in 1733 total slices. A post-reconstruction ring correction was applied to slices 1420-1733. Segmentation, image processing, and surface-file editing were accomplished using VGStudio Max (version 2.0.1) and Meshmixer (version 3.3.15). 3D prints of the specimen were made by a Formlabs Form 1+ to help visualize the inner ears and cranial nerve pathways. Videos of 3D volumes can be accessed in the Supplemental Data.

Phylogenetic analyses. - The phylogenetic analyses were conducted using a character-taxon matrix constructed by Nesbitt et al. (2009b) and modified by Ezcurra and Brusatte (2011), Nesbitt and Ezcurra (2015), and Marsh et al. (2019) who added to the states of three characters (numbers 336, 342, and 343 of this study), rescored taxa for those characters, and added seven characters (characters 346-352). In the current study, we add seven additional characters (353-359) and states to four characters $(32,38,102,131)$. We did not include certain theropods such as Dracoraptor hanigani Martill et al., 2016, Dracovenator regenti (Yates, 2005), Tachiraptor admirabilis (Langer et al., 2014), Sinosaurus triassicus (Young, 1940; Hu, 1993), and Shuangbaisaurus anlongbaoensis Wang et al., 2017 because they have not been scored in this matrix and we have not seen those specimens in person. Character descriptions and the data matrix are located in the Supplemental Data. In order to avoid the chimeric scoring of Dilophosaurus wetherilli used in previous studies and to test the monophyly of the taxon, we scored five specimens as operational taxonomic units (UCMP 37302, UCMP 37303, UCMP 77270, TMM 43646-1, TMM 47006-1) and ran a total of six equal-weight parsimony analyses in TNT (Goloboff et al., 2008) using the matrix constructed in Mesquite (Maddison and Maddison, 2015) of 
359 characters and heuristic searches, estimating Wagner trees using 1,000 repetitions and randomly taxon addition sequences before tree bisection and reconnection branch swapping. Characters 17, 30, 67, 128, 174, 184, 213, 219, 231, 236, 248, $253,254,273,329$, and 343 were ordered, and all characters were weighted equally. Ten trees were held for each replicate and zero-length branches were collapsed (Coddington and Scharff, 1994). The first analysis included only the holotype of Dilophosaurus wetherilli (UCMP 37302), the second included the holotype and paratype (UCMP 37303), the third included the holotype and large UCMP specimen (UCMP 77270), the fourth included the holotype and the large TMM specimen (TMM 43646-1), the fifth included the holotype and the small TMM specimen (TMM 47006-1), and the sixth included all five specimens. Character descriptions, TNT files, and apomorphy lists for each analysis are found in the Supplemental Data.

We prefer to retain the generic name 'Syntarsus' for the South African Syntarsus rhodesiensis Raath, 1969 and North American Syntarsus kayentakatae instead of 'Megapnosaurus' (Ivie et al., 2001) or 'Coelophysis' (Bristowe and Raath, 2004) because the systematic relationships of these animals within Coelophysoidea is in flux. Congenericity or the need of an additional genus name (e.g., Megapnosaurus) is not supported if Coelophysis bauri, Syntarsus rhodesiensis, or Syntarsus kayentakatae do not form respective clades. We refrain from nomenclatural acts when interpreting the phylogenetic results below. Discussion on the phylogenetic context of genera is provided elsewhere (de Queiroz and Gauthier, 1992; Gill et al., 2005; Stuessy, 2009; Parker, 2018).

Vertebral nomenclature.-We refer to the structural bony laminae and the associated fossae found on the vertebral neural arches using terminology that was originally erected for the description of sauropod vertebrae (Osborn, 1899; Osborn and Mook, 1921; Janensch, 1929; Bonaparte, 1999; Wilson, 1999; Harris, 2006; Wilson et al., 2011, 2012). This nomenclature was adopted for other archosaurs under the assumption that a given structure is homologous among archosaur groups (Wilson, 1999, 2012; Parker, 2008; Nesbitt, 2011; Wilson et al., 2011, 2016). The vertebral laminae are named for the two landmarks that they connect; for example, the prezygadiapophyseal laminae connects the prezygapophysis and the diapophysis (Wilson, 1999). The vertebral fossae are named for the two or three landmarks that demarcate a given fossa; for example, the prezygapophyseal centrodiapophyseal fossa is found between the prezygapophysis and diapophysis and is bounded by the prezygadiapophyseal lamina and anterior centrodiapophyseal lamina (Wilson et al., 2011). Nine of Welles' (1984) 'chonoses' correspond to some of these vertebral fossae; we use the more recent terminology (e.g., Wilson, 1999; see Wilson et al., 2011, table 1 comparing some of these names) because it relies on a landmark-based system that allows for the description of more concavities and direct comparisons among other archosaurs. We number the vertebrae anterior to posterior and do not restart the numbering after the presacral series. We prefer to identify the cervical vertebrae as those that retain the parapophysis on the centrum rather than the neural arch, and have a ventral keel, which is a standard definition of that region for early dinosaurs. The parapophysis shifts from the anterodorsal corner of the centrum to the anteroventral margin of the neural arch after the fourteenth presacral vertebra (e.g., UCMP 37320 and TMM 47006-1). This results in the unusual condition where Dilophosaurus wetherilli has 14 cervical vertebrae, not the ten present plesiomorphically. This does not necessarily mean that all 14 vertebrae were found in the neck, but that cervical vertebrae 11 , 12,13 , and 14 resemble the anterior presacral vertebrae more than the posterior presacral vertebrae. Given the unique anatomy of the first 14 vertebrae of Dilophosaurus wetherilli, we refrain from calling presacral vertebrae 10-13 "pectoral vertebrae" (Welles, 1984, p. 109) because the parapophysis is never simultaneously on the centrum and the neural arch.

Repositories and institutional abbreviations.- $\mathrm{ARCH}$, Arches National Park, UT; MNA, Museum of Northern Arizona, Flagstaff, AZ; PEFO, Petrified Forest National Park, AZ; TMM, Texas Vertebrate Paleontology Collections, The University of Texas at Austin, Austin, TX; UCMP, University of California Museum of Paleontology, Berkeley, CA.

\section{Systematic paleontology}

Archosauria Cope, 1869 sensu Sereno, 2005

Dinosauria Owen, 1842 sensu Sereno, 2005

Saurischia Seeley, 1888 sensu Sereno, 2005

Theropoda Marsh, 1881 sensu Sereno, 2005

Genus Dilophosaurus Welles, 1970

Type species.—Dilophosaurus wetherilli (Welles, 1954) by monotypy.

Formerly included species._-Dilophosaurus sinensis' $\mathrm{Hu}$ 1993 was assigned to the genus Dilophosaurus owing to it having a pair of parasagittal cranial crests. This taxon is now assigned to Sinosaurus triassicus Young, 1940 (Carrano et al., 2012), and probably represents a basal tetanuran (Hendrickx and Mateus, 2014) and should not be referred to the genus Dilophosaurus.

Diagnosis.-As for type species by monotypy.

Occurrence.-Silty Facies of the Kayenta Formation, Early Jurassic, Sinemurian-Pliensbachian (Marsh et al., 2014), Navajo Nation, Coconino County, Arizona. The UCMP material is from fairly low in section $(\sim 10 \mathrm{~m}$ above the contact with the Moenave Formation) and potentially older than the TMM material, which is considered from the 'middle third' of the Silty Facies at Gold Spring, AZ. That chronological relationship has been corroborated by U-Pb detrital zircon geochronology (Marsh, 2014). See below for further locality information. Detailed locality information and historical field notes are available at the UCMP, TMM, and MNA to qualified researchers.

\section{Dilophosaurus wetherilli (Welles, 1954)} Figures 3-64, 66

1954 Megalosaurus wetherilli Welles, p. 591. 
1955 Megalosaurus sp. Swinton, p. 133.

1965 Megalosaurus wetherilli; Charig, Attridge, and Crompton, p. 216.

1970 Megalosaurus wetherilli; Steel, p. 35.

1970 Dilophosaurus wetherilli Welles, p. 989.

Holotype.-UCMP 37302 (Welles, 1954). Partial skull and postcranial skeleton (see below).

Diagnosis.—See revised diagnosis in Discussion below.

Occurrence.-UCMP V4214, Moa Ave 1 near Tuba City, AZ. Lower part of the Kayenta Formation, Silty Facies (see below).

Description and materials.-The UCMP and TMM specimens are assigned to the type and only species of Dilophosaurus, Dilophosaurus wetherilli, based on the autapomorphies in the revised diagnosis of this study, and the MNA and $\mathrm{ARCH}$ specimens are referred to as cf. Dilophosaurus wetherilli.

University of California Museum of Paleontology, Berkeley, CA.-The UCMP houses the three individuals collected by Sam Welles in 1942 and 1964, including the holotype and paratype specimens (Welles, 1954, 1970). The third larger specimen was collected 20 years later than the holotype and paratype skeletons, but all three purportedly come from the same stratigraphic horizon (Welles, 1984). We were able to verify the location of the holotype quarry (UCMP V4214), but not that of UCMP 77270 (V6468). According to maps at the UCMP, the two sites are not more than a few miles apart.

UCMP 37302 (holotype specimen).-Locality UCMP V4214, Moa Ave 1, Coconino County, Navajo Nation, Arizona, near the famous Tuba City dinosaur tracks south of the town of Moenave, AZ, low in section, nearly $10 \mathrm{~m}$ above the contact with the Dinosaur Canyon Member of the Moenave Formation (Marsh, unpublished data, 2015; Fig. 1). The skeleton was articulated in an opisthotonic position in the quarry, and Langston's panel mount reconstructed this with some fidelity; the neck was straightened and the right leg was positioned up above the pelvis in order to better display it (Welles, 1954). Most of the preserved cranial material is contained within a single block of bones that remain firmly adhered to one another (Fig. 3). The right side of the skull is articulated and contains the posterior half of the maxilla, the complete jugal and quadratojugal, the quadrate, the squamosal, the postorbital, and the lacrimal. The bones on the other side of this larger block are crushed and difficult to identify. The posteroventral portion of the left maxilla is found just below the level of the right maxilla, and thin bones above that region may belong to the palate. Isolated cranial elements from the left side include the incomplete lacrimal, postorbital, squamosal, quadratojugal, and quadrate. The left nasal and lacrimal are preserved in isolation and include the ventral portion of the crest. An element identified with a written tag by Welles as the left ectopterygoid is also preserved. Braincase elements are preserved in one piece, but are crushed from the left side, making it difficult to identify individual bones or regions (Fig. 4). The basioccipital and parabasisphenoid are mostly complete, and the otoccipitals are not coossified to the basioccipital. The paroccipital processes are broken distally, and only the right side of the supraoccipital remains. The lower jaws are represented in two pieces (Fig. 5). The left surangular is missing its anterior third portion, but the bone is articulated with the prearticular, articular, and angular. Right mandibular elements comprise the partial prearticular and surangular, which are stuck to the medial side of the preserved middle portion of left dentary.

Most of the individual elements of the vertebral column are represented by either the centrum or neural arch anterior to the trunk, however, the serial position of each element is confirmed by the articulated nature of the skeleton. The atlantal pleurocentrum (odontoid) and intercentrum are separate elements and are articulated to the front of the axis (Fig. 6). The proatlas and left atlantal neural arch are complete, but the right arch is missing. The axis intercentrum is separate, but articulated to the pleurocentrum. The cervical neural arches are not coossified to their centra. Welles (1984) mentioned that the third cervical is crushed into the back of the left mandible, but it no longer is attached there and must have been removed. A small piece of neural arch labeled " 3 " is in the drawer with the rest of the cervical vertebrae, but it is too fragmentary to describe. Most of the neural arch and left side of the centrum are preserved in cervical vertebrae 4 and 6 (Figs. 7-9). Presacral vertebrae 5 and 7 comprise only the top of the neural arch and the posterodorsal part of the centrum. The eighth presacral vertebra is only a neural arch, but the left prezygapophysis and postzygapophyses were not included in the restoration and are isolated elements. The same is true for the right transverse process of presacral vertebra 9 , which otherwise only exists as the posterior half of the centrum.

The trunk vertebrae of the holotype are better preserved than the cervical vertebrae and most of them comprise both the neural arch and centrum (Fig. 10). The two elements have been plastered together throughout the trunk series, because they are not coossified. The neural spines are missing on presacral vertebrae $20,21,23$, and 24 . The same vertebrae are also missing most of their neural arches. Presacral vertebra 24 is especially fragmentary and only preserves the front of the centrum and the left posterolateral side of the neural arch. Vertebra 24 is most likely the last presacral vertebra, but may have been incorporated in the anterior region of the sacrum. Welles (1984) described it as the last trunk vertebra, but Tykoski (2005) identified it as the anterior of two dorsosacral vertebrae. At the time, Dilophosaurus wetherelli and coelophysoids were hypothesized to be ceratosaurians and thus may have incorporated more than one trunk vertebra into the sacrum. We prefer to maintain a more conservative identification by assuming the incorporation of one dorsosacral anterior to primordial sacral 1, the rib of which is consistent in shape with that of other saurischian dinosaurs. Thus, we follow Welles (1984) in calling vertebra 24 the last presacral vertebra, which is supported by counting vertebrae in the articulated panel mounts (Welles, 1954).

Traditionally, the number of vertebrae in the sacrum has been determined by back-calculating from the number of presacral vertebrae, matching the medial scars of the ilium to sacral ribs or transverse processes (Tykoski, 2005), or identifying 'primordial sacrals' (Nesbitt, 2011). The presacral vertebral column is seemingly complete and was restored that way for Langston's original wall mount, but there are elements that comprise only centra and others that comprise only the neural arch. The 


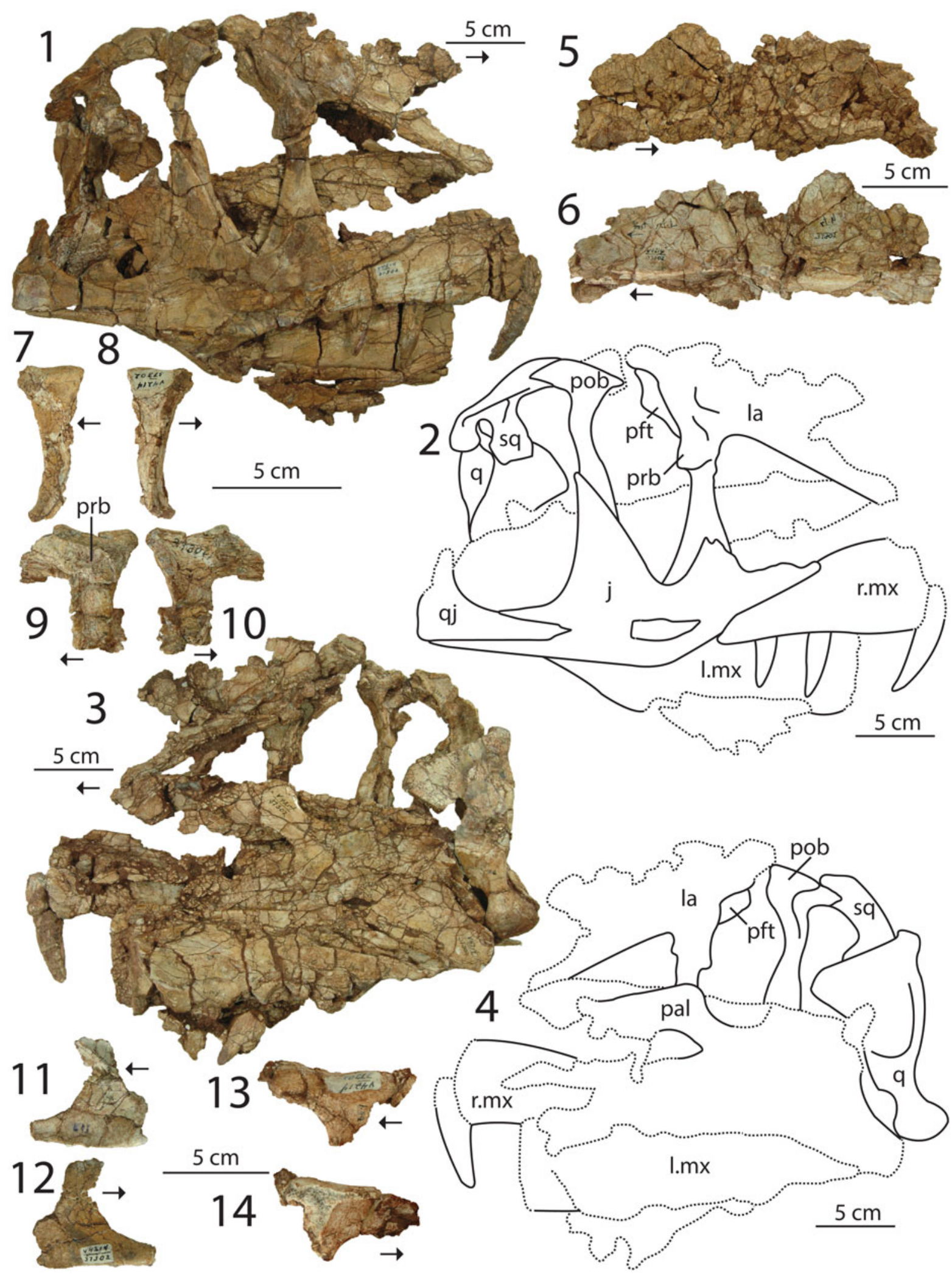

Figure 3. Dilophosaurus wetherilli holotype specimen (UCMP 37302): (1-4) articulated right side of the skull, $(\mathbf{5}, \mathbf{6})$ nasolacrimal crest, $(\mathbf{7}, \mathbf{8})$ left postorbital, $(\mathbf{9}$, 10) left lacrimal, $(\mathbf{1 1}, \mathbf{1 2})$ left quadratojugal, and $(\mathbf{1 3}, \mathbf{1 4})$ left squamosal in $(\mathbf{1}, \mathbf{2 , 5 , 7 , 9 , 1 1 , 1 3 )}$ lateral and $(\mathbf{3 , 4 , 6 , 8 , 1 0 , 1 2 , 1 4 )}$ medial view. Arrows point in anterior direction. $\mathrm{j}=$ jugal; la = lacrimal; $1 \mathrm{mx}=$ left maxilla; $\mathrm{q}=$ quadrate; $\mathrm{qj}=$ quadratojugal; pal = palatine; $\mathrm{pft}=$ prefrontal; pob = postorbital; prb = preorbital boss; $\mathrm{r} . \mathrm{mx}=$ right maxilla; sq = squamosal 

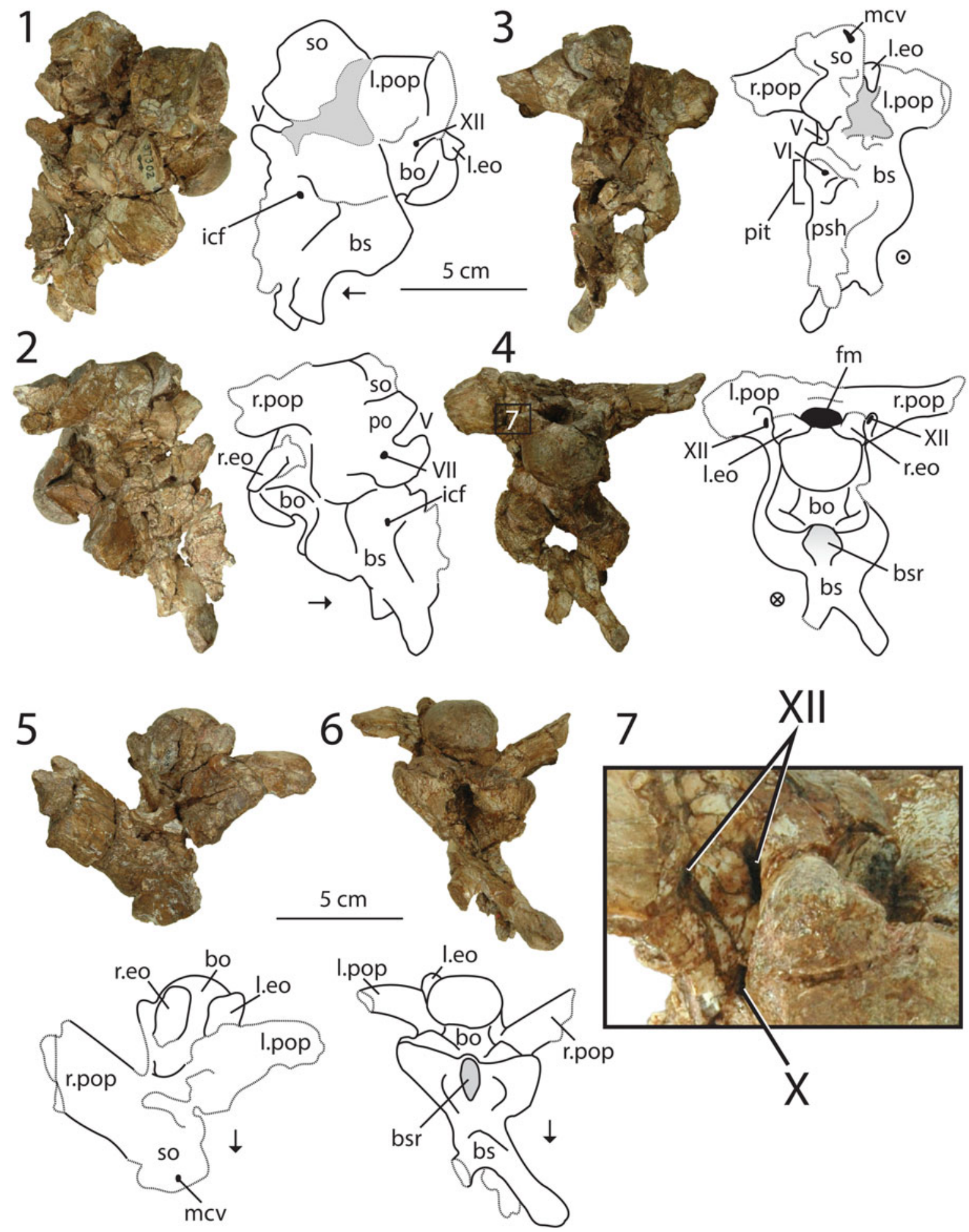

Figure 4. Dilophosaurus wetherilli holotype specimen (UCMP 37302): (1-7) braincase with interpretive drawings in (1) left lateral, (2) right lateral, (3) anterior, $(\mathbf{4}, \mathbf{7})$ posterior, (5) dorsal, and (6) ventral view. Arrows point in anterior direction. Inset box on (4) shows location of $(\mathbf{7})$. bo = basioccipital; bs = basisphenoid; $\mathrm{bsr}=$ basisphenoid recess; $\mathrm{fm}=$ foramen magnum; icf $=$ interal carotid foramen; $1 . e o=$ left exoccipital; 1 pop = left paroccipital process of the opisthotic; $\mathrm{mcv}=$ foramen for the middle cerebral vein; pit = pituitary fossa; $p o=$ prootic; $p s h=$ parasphenoid; r.eo=right exoccipital; r.pop=right paroccipital process of the opisthotic; so = supraoccipital; $\mathrm{V}=$ opening for the trigeminal nerve; $\mathrm{VI}=$ foramen or canal for the abducens nerve; $\mathrm{VII}=$ foramen or canal for the facial nerve; $\mathrm{X}=$ foramen or canal for the vagus nerve; XII = foramen or canal for the hypoglossal nerve.

supposed last presacral (vertebra 24) is highly fragmentary, but Tykoski (2005) thought it represents the anterior of two trunk vertebrae incorporated into the sacrum (see above). Four vertebrae articulate with one another behind it and we will describe them with their number as assigned by Welles (1984). Vertebrae 25 and 26 are centra and crushed anterior 

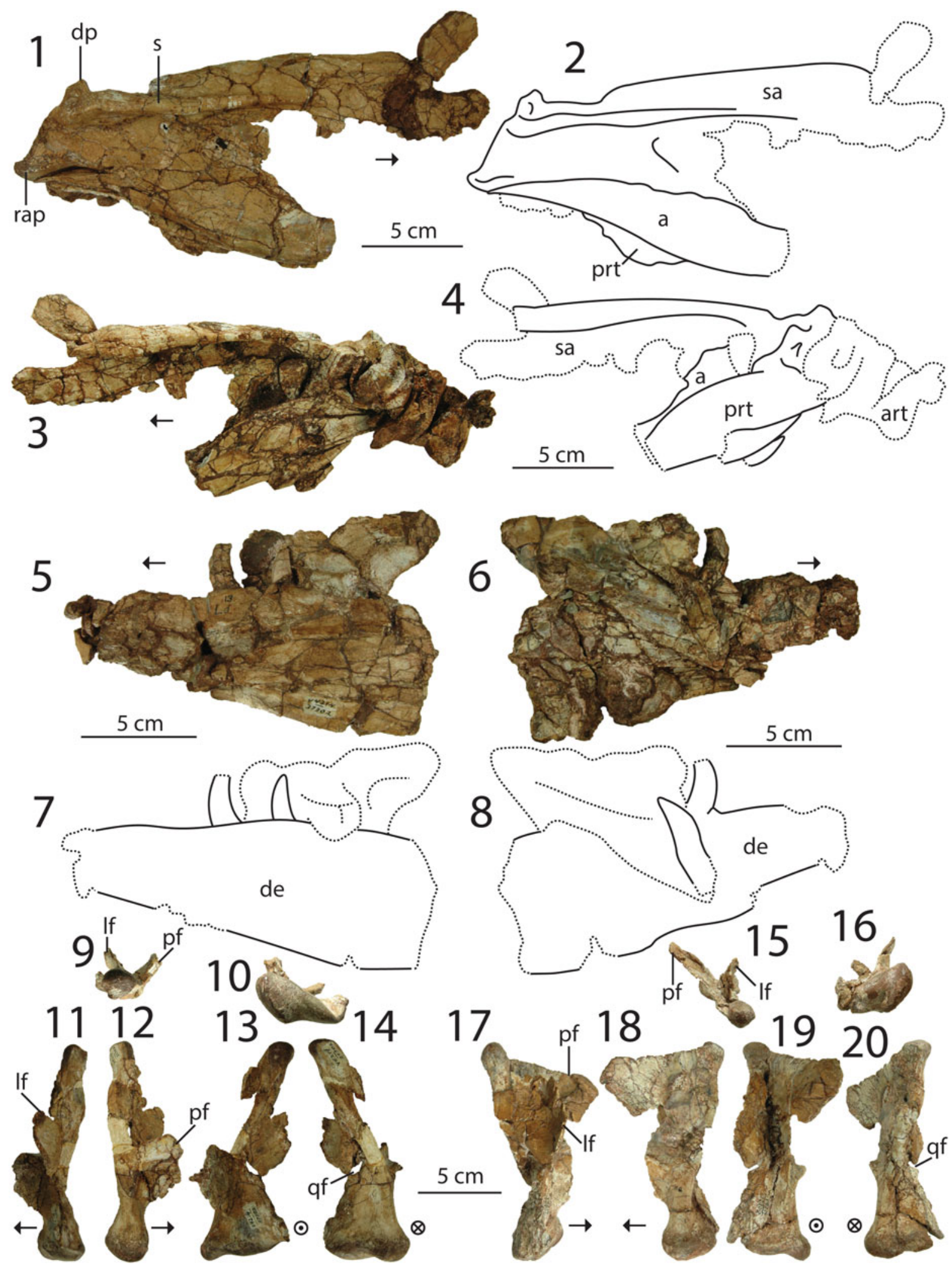

Figure 5. Dilophosaurus wetherilli holotype specimen (UCMP 37302): (1-4) articulated partial right mandible, (5-8) partial left dentary, (9-14) left quadrate, and $(\mathbf{1 5}-\mathbf{2 0})$ right quadrate in $(\mathbf{1}, \mathbf{2}, \mathbf{5}, \mathbf{7}, \mathbf{1 1}, \mathbf{1 7})$ lateral, $(\mathbf{3}, \mathbf{4}, \mathbf{6}, \mathbf{8}, \mathbf{1 2}, \mathbf{1 8})$ medial, $(\mathbf{9}, \mathbf{1 5})$ dorsal, $(\mathbf{1 0}, \mathbf{1 6})$ ventral, $(\mathbf{1 3}, \mathbf{1 9})$ anterior, and $(\mathbf{1 4}, \mathbf{2 0})$ posterior view. Arrows point in anterior direction. $\mathrm{a}=$ angular; art $=$ articular; $\mathrm{de}=$ dentary; $\mathrm{dp}=$ dorsal process; $\mathrm{lf}=$ lateral flange; $\mathrm{pf}=$ pterygoid flange; $\mathrm{prt}=$ prearticular; $\mathrm{qf}=\mathrm{quadrate}$ foramen; rap = retroarticular process; $\mathrm{s}=$ shelf; $\mathrm{sa}=$ surangular. 

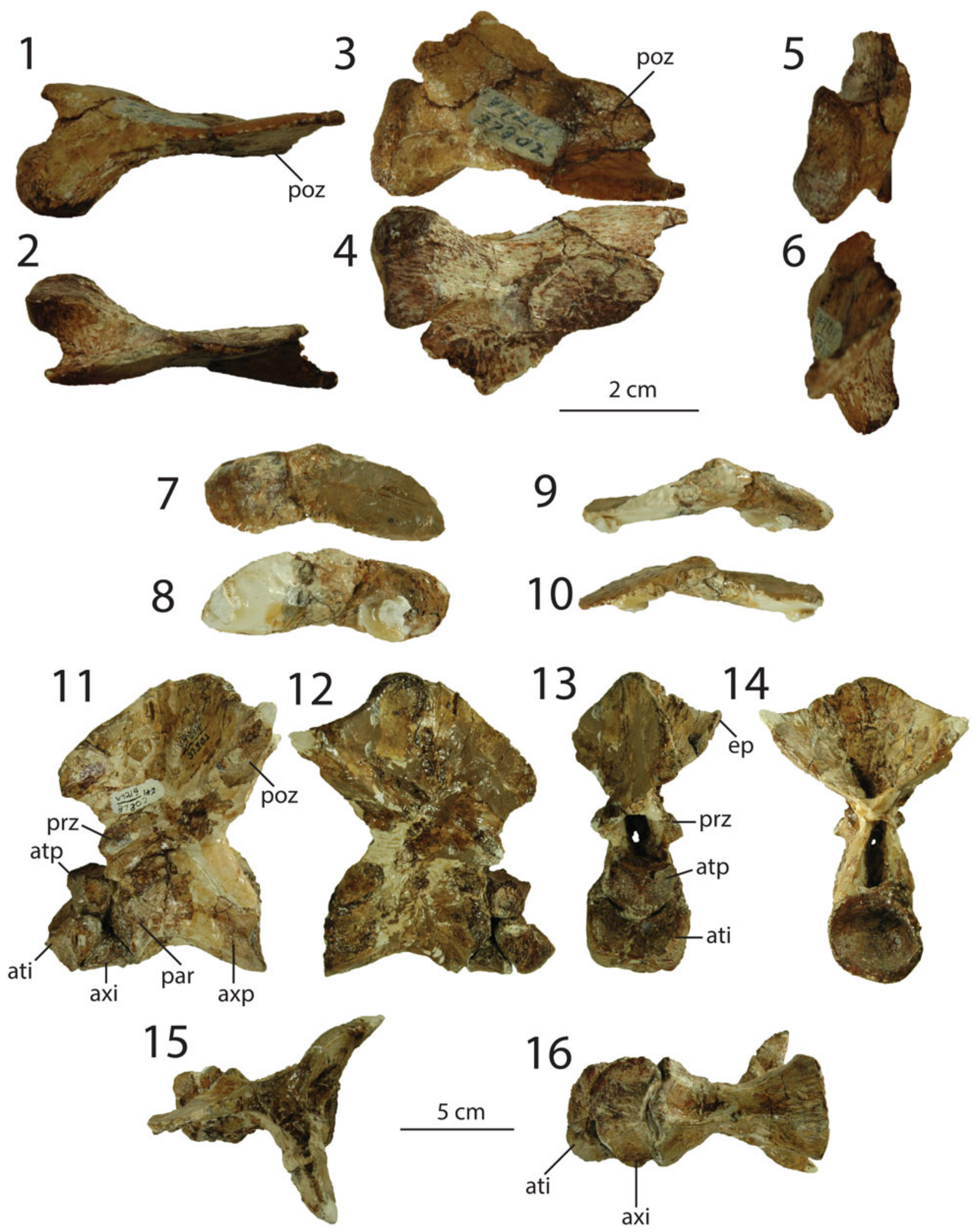

Figure 6. Dilophosaurus wetherilli holotype specimen (UCMP 37302): (1-6) left neurapophysis, (7-10) proatlas, and (11-16) atlas-axis in (1, 7, 15) dorsal, $(\mathbf{2 , ~ 8}$, 16) ventral, $(3,11,12)$ lateral, $(4)$ medial, $(5,9,13)$ anterior, and $(6,10,14)$ posterior view. ati $=$ atlantal intercentrum; atp = atlantal pluerocentrum; axi $=$ axial intercentrum; $\operatorname{axp}=$ axial pluerocentrum; $\mathrm{ep}=$ epipophysis; $\mathrm{par}=$ parapophysis; $\mathrm{poz}=$ postzygapophysis; prz = prezygapophysis.

neural arches (Fig. 11). The neural arch of vertebra 27 is better preserved, but broken dorsally. Vertebra 28 is complete and preserves the transverse processes and neural spine. Judging by the estimated length of the ilia and the articulation of 

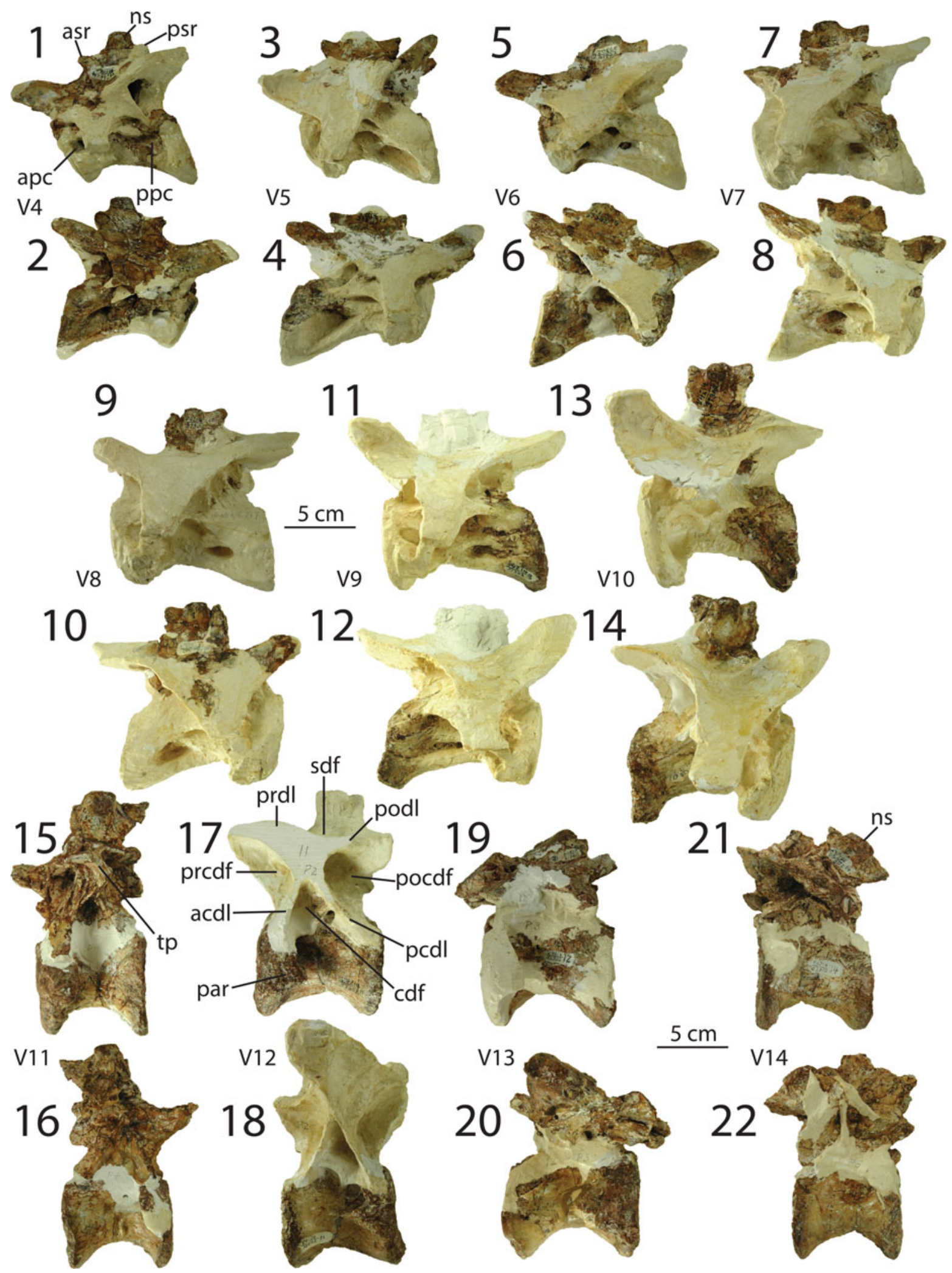

Figure 7. Dilophosaurus wetherilli holotype specimen (UCMP 37302): (1-22) cervical vertebrae (fourth through $\left.14^{\text {th }} ; \mathrm{V} 4-\mathrm{V} 14\right)$ in $(\mathbf{1}, \mathbf{3}, \mathbf{5 , 7}, \mathbf{9}, \mathbf{1 1}, \mathbf{1 3}, \mathbf{1 5}, \mathbf{1 7}, \mathbf{1 9}$, 21 $)$ left lateral and $(\mathbf{2}, 4,6,8,10,12,14,16,18,20,22)$ right lateral view. acdl $=$ anterior centrodiapophyseal lamina; apc $=$ anterior pluerocoel; asr $=$ anterior shoulder; $\mathrm{cdf}=$ centrodiapophyseal fossa; $\mathrm{ns}=$ neural spine; par = parapophysis; pcdl = posterior centrodiapophyseal lamina; pocdf = postzygapophyseal centrodiapophyseal fossa; podl = postzygadiapophyseal lamina; ppc = posterior pluerocoel; predf = prezygapophyseal centrodiapophyseal fossa; prdl = prezygadiapophyseal lamina; $\mathrm{psr}=$ posterior shoulder; $\mathrm{sdf}=$ spinodiapophyseal fossa; $\mathrm{tp}=$ transverse process; $\mathrm{v}=$ vertebra . 

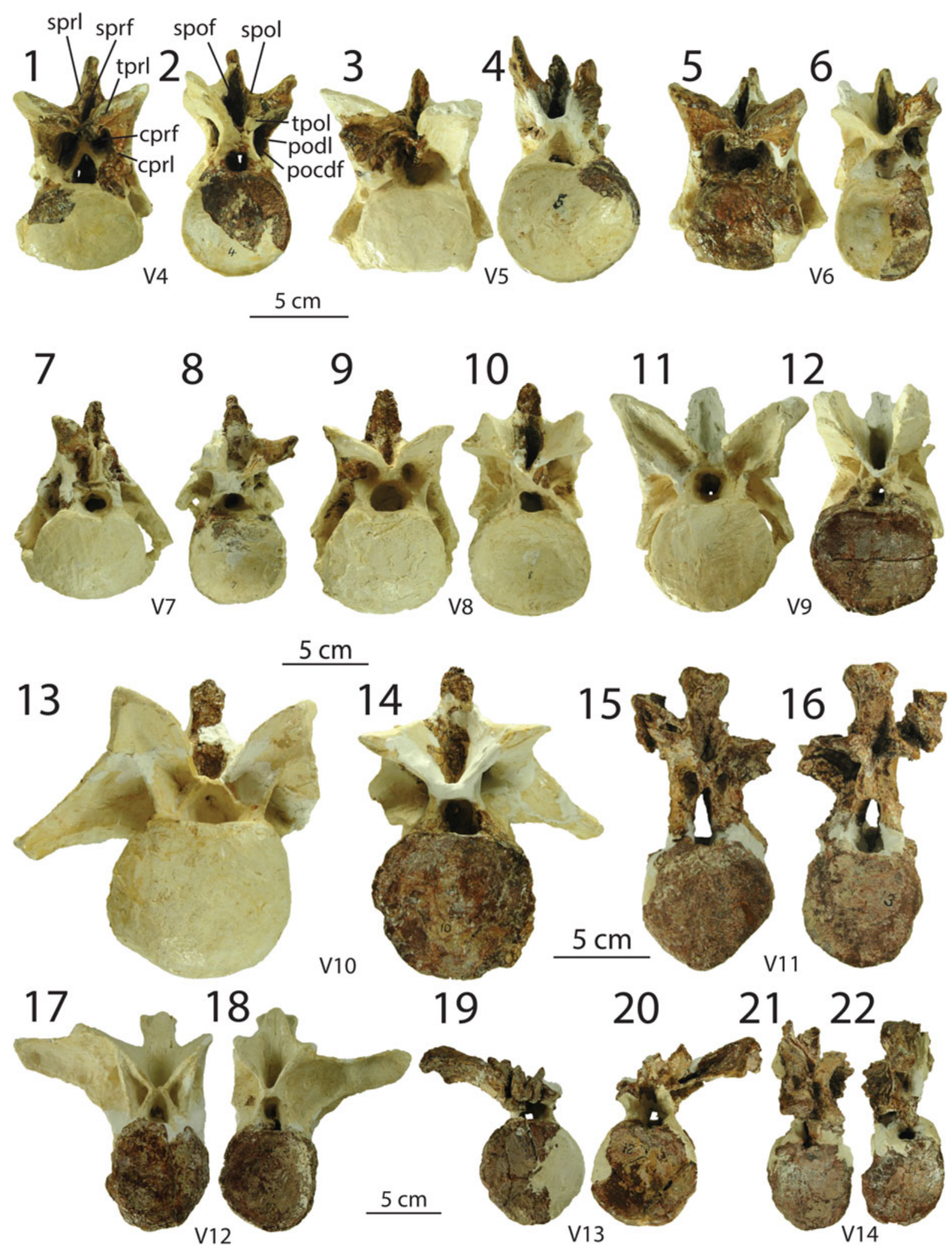

19

Figure 8. Dilophosaurus wetherilli holotype specimen (UCMP 37302): (1-22) cervical vertebrae (fourth through $\left.14^{\text {th }} ; \mathrm{V} 4-\mathrm{V} 14\right)$ in $(\mathbf{1 , 3}, \mathbf{5}, \mathbf{7 , 9}, \mathbf{1 1}, \mathbf{1 3}, \mathbf{1 5}, \mathbf{1 7}, \mathbf{1 9}$ 21) anterior and $(2,4,6,8,10,12,14,16,18,20,22)$ posterior view. cprf = centroprezygapophyseal fossa; cprl = centroprezygapophyseal lamina; pocdf = postzygapophyseal centrodiapophyseal fossa; podl = postzygadiapophyseal lamina; spof = spinopostzygapophyseal fossa; spol = spinopostzygapophyseal lamina; sprf = spinoprezygapophyseal fossa; $\mathrm{sprl}=$ spinoprezygapophyseal lamina; tpol = infrapostzygapophyseal lamina; tprl = infraprezygapophyseal lamina; $\mathrm{v}=\mathrm{vertebra}$. 


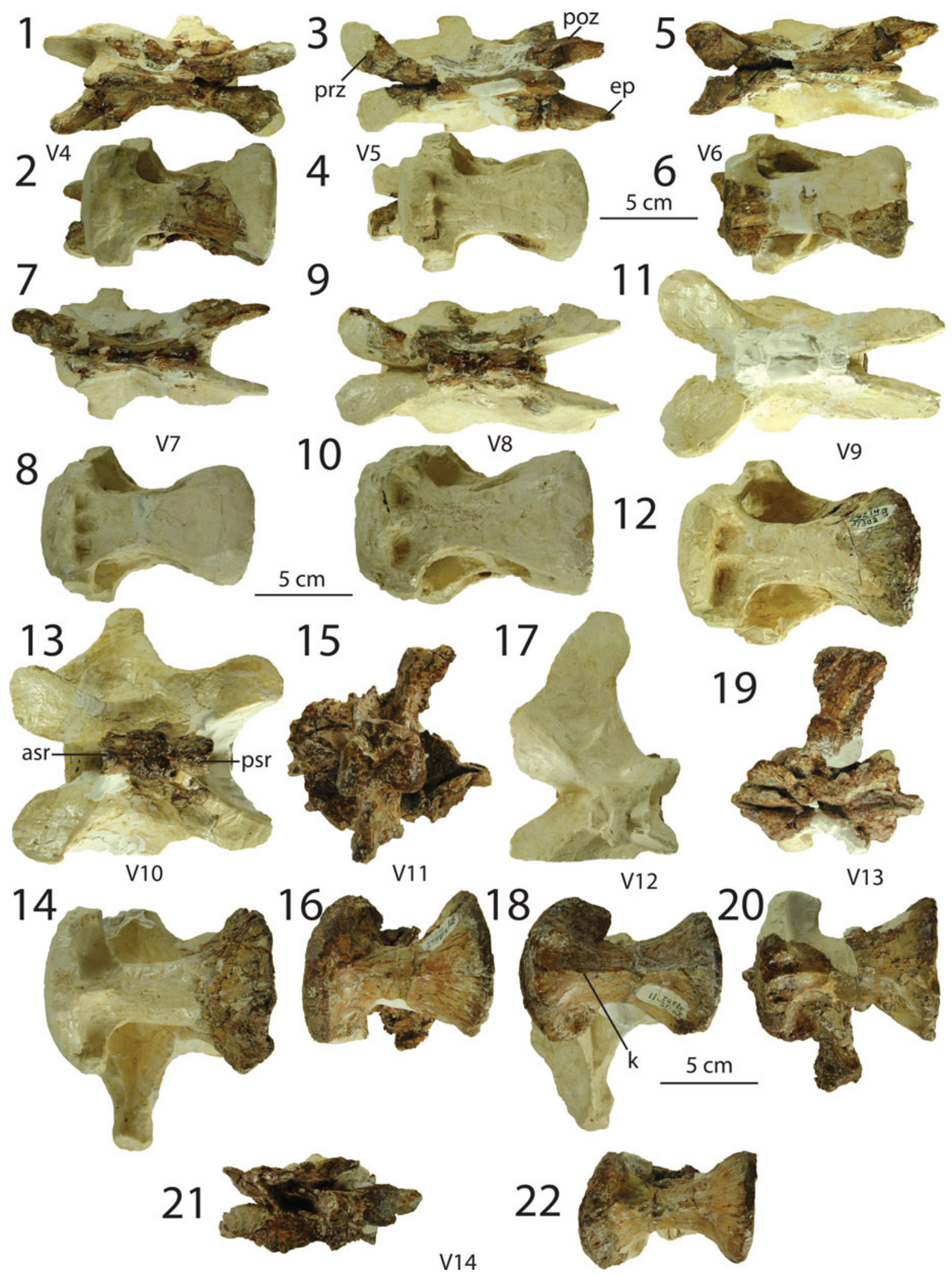

Figure 9. Dilophosaurus wetherilli holotype specimen (UCMP 37302): (1-22) cervical vertebrae (fourth through $14^{\text {th }} ;$ V4-V14) in $(\mathbf{1}, \mathbf{3}, \mathbf{5 , 7}, \mathbf{9}, \mathbf{1 1}, \mathbf{1 3}, \mathbf{1 5}, \mathbf{1 7}, \mathbf{1 9}$, 21) dorsal and $(\mathbf{2}, \mathbf{4}, \mathbf{6}, \mathbf{8}, \mathbf{1 0}, \mathbf{1 2}, \mathbf{1 4}, \mathbf{1 6}, \mathbf{1 8}, \mathbf{2 0}, \mathbf{2 2})$ ventral view. Anterior is to the left. asr = anterior shoulder; ep = epipophysis; $\mathrm{k}=\mathrm{keel}$; poz = postzygapophysis; prz = prezygapophysis; $\mathrm{psr}=$ posterior shoulder; $\mathrm{v}=$ vertebra.

vertebrae 25-28, we agree with Welles (1984) in preliminarily attributing only four vertebrae to the sacral series. Using the identification of the two primordial sacrals based on the shapes of their ribs (Nesbitt, 2011), vertebra 26 is primordial sacral 1 


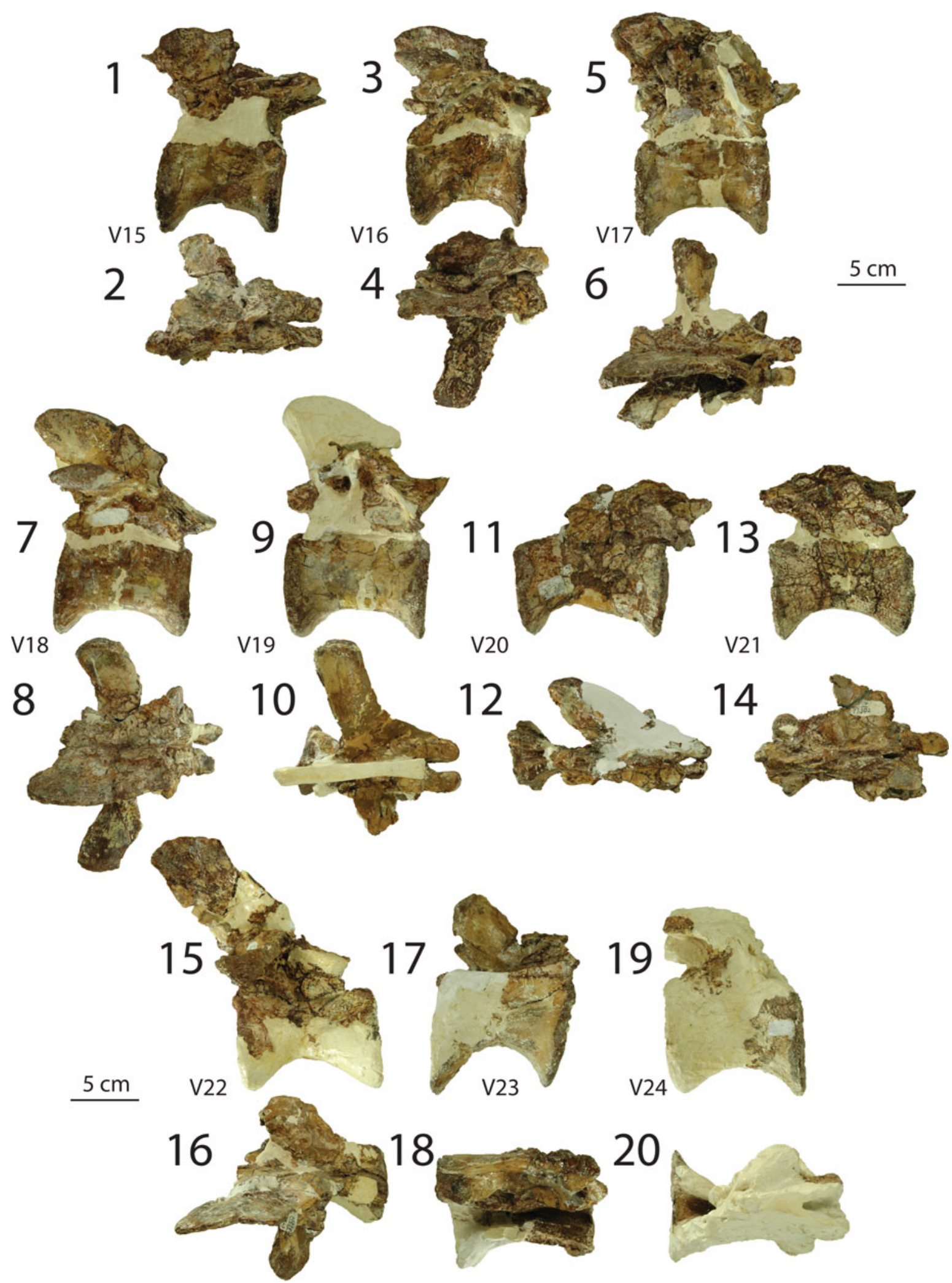

Figure 10. Dilophosaurus wetherilli holotype specimen (UCMP 37302): (1-20) trunk vertebrae (vertebrae 15-24; V15-V24) in $(\mathbf{1}, \mathbf{3}, \mathbf{5}, \mathbf{7}, \mathbf{9}, \mathbf{1 1}, \mathbf{1 3}, \mathbf{1 5}, \mathbf{1 7}, \mathbf{1 9})$ right lateral and $(\mathbf{2}, \mathbf{4}, \mathbf{6}, \mathbf{8}, \mathbf{1 0}, \mathbf{1 2}, \mathbf{1 4}, \mathbf{1 6}, \mathbf{1 8}, \mathbf{2 0})$ dorsal view. Anterior is to the right. v = vertebra

and vertebra 27 is primordial sacral 2. This means that any vertebrae incorporated into the sacrum on either side of these (i.e., vertebrae 25 and 28) are modified trunk or tail vertebrae.
The caudosacral vertebra (vertebra 28) exhibits an open neural arch-centrum suture, but this area is coossified near the base of the transverse process. 


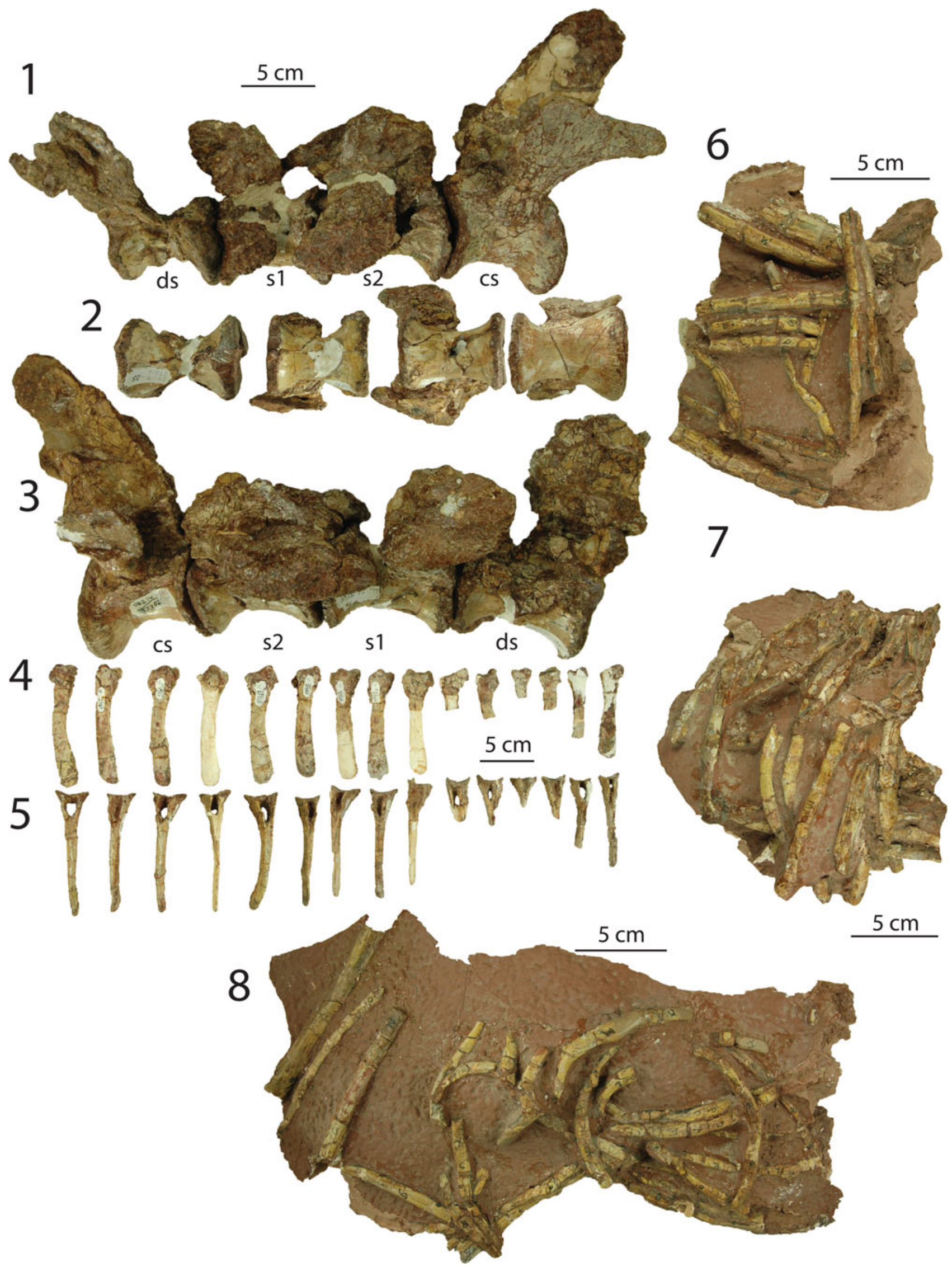

Figure 11. Dilophosaurus wetherilli holotype specimen (UCMP 37302): (1-3) sacral vertebrae (vertebrae 24-28), (4, 5) haemal arches, and (6-8) three blocks of gastralia in $(\mathbf{1}, \mathbf{4})$ left lateral, $(\mathbf{2})$ ventral, $(\mathbf{3})$ right lateral, and (5) anterior view. cs = caudosacral vertebra; ds = dorsosacral vertebra; $\mathrm{s}=$ sacral vertebra. 


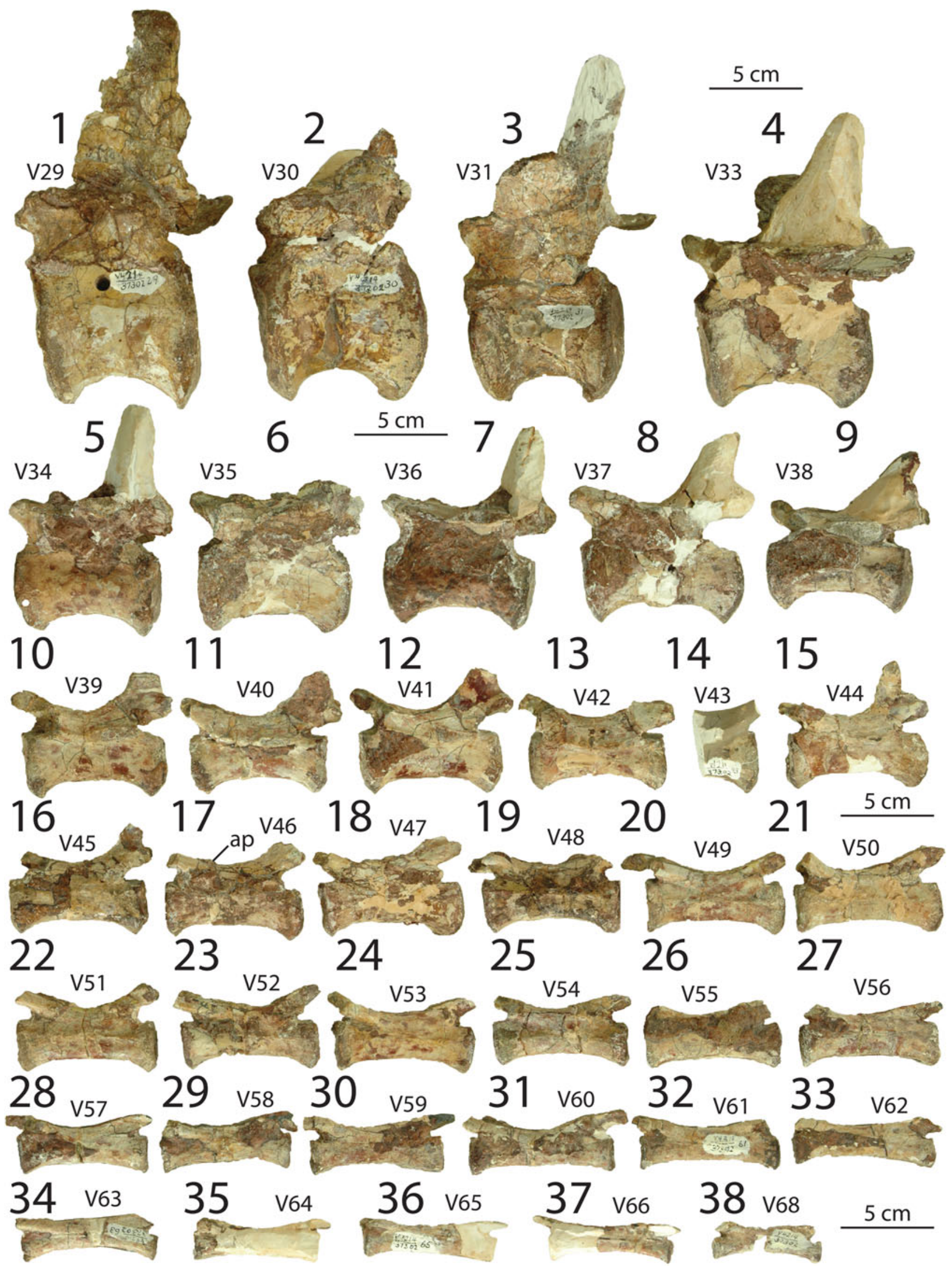

Figure 12. Dilophosaurus wetherilli holotype specimen (UCMP 37302): (1-38) caudal vertrebrae (vertebrae 29-68; V29-V68) in (1-38) left lateral view. Caudal vertebrae 32 and 67 were entirely reconstructed in plaster and are not figured. Anterior is to the left. ap = anterior shoulder; $v=$ vertebra. 

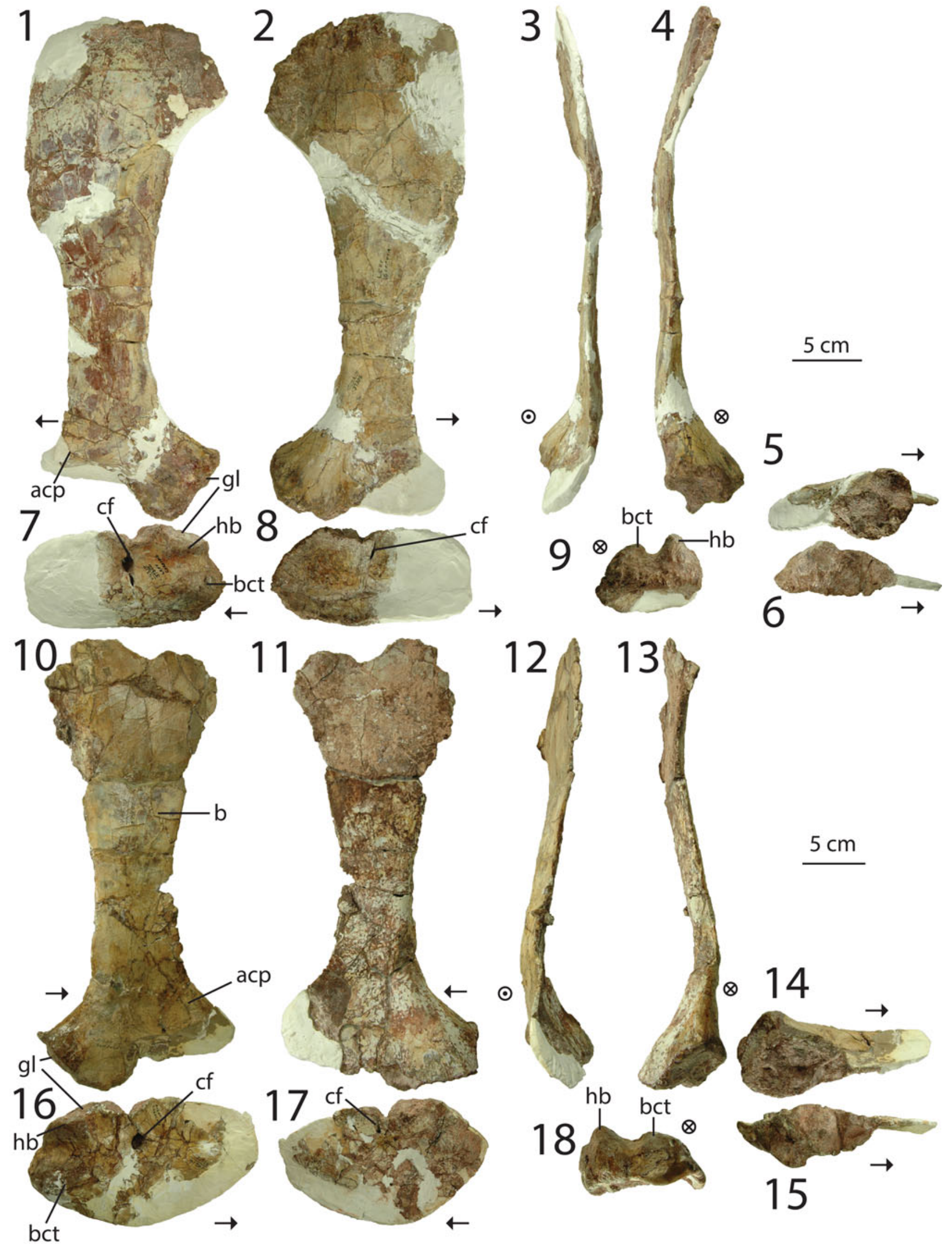
Our count of the number of caudal vertebrae present is inconsistent with what Welles (1984) reported. Instead of 44 vertebrae, only 38 are present. The first caudal vertebra (that is, the first that is not incorporated into the sacrum) is vertebra 29 (Fig. 12). To minimize confusion, we will retain the numbered system that is written on each element so that the preserved tail of the holotype spans vertebrae 29 through 68 , where vertebrae 32 and 67 are entirely reconstructed in plaster and paint and are not described or figured here. The caudal series almost certainly included more than these 38 vertebrae, but the total length and number of elements is impossible to determine. In order to accommodate the bas-relief of the original wall mount figured in Welles (1954), the left side of many of the caudal vertebrae were altered; most of the left transverse processes were broken off and many of the left prezygapophyses are also missing. The neural spines of vertebrae 30-38 are reconstructed and vertebra 43 comprises only the back end of the centrum. The sutures between the neural arches and centra of the anterior caudal vertebra are visible until vertebra 38 .

As Welles (1984) noted, the holotype specimen does not preserve any cervical ribs. Many fragmentary thoracic ribs are present, but most of the proximal ends are missing. Three blocks include associated gastralia, but their arrangement is unknown (Fig. 11). The first 12 or so haemal arches are present and mostly complete.

The holotype specimen has an incomplete pectoral girdle (Fig. 13); the scapulae and coracoids are not coossified with one another, and the sternal plates and furcula were not preserved (although the presence of sternal plates in early theropods has not been documented except for those of Tawa hallae; Bradley et al., 2020). The scapulae and coracoids are missing their anterior margins near their glenoids. The humeri, radii, and ulnae are complete, although somewhat crushed. The distal end of the right humerus is rotated laterally, the distal left ulna is rotated medially, and both ends of the right radius are worn (Figs. 14, 15). The holotype carpus only includes a single element on the right side that is referable to distal carpal 1 of other saurischians (Fig. 16). Welles (1984) mentioned the presence of three carpals, but the dimensions given do not match that of the element from the right side. The left manus is mostly complete and preserves four digits (Fig. 17). The right manus includes metacarpals I-III and a few proximal phalanges, and the ungual of digit II, but most of the manus is reconstructed. The pathological nature of the forelimbs of this individual is documented elsewhere (Senter and Juengst, 2016).

The pelvic girdle is incomplete, but preserves parts of all three elements from both sides. The left ilium is missing its anterior margin from the middle of the acetabulum forwards (Fig. 18.1-18.7). The right ilium consists only of the pubic peduncle. Both ilia have been restored, first after Allosaurus fragilis Marsh, 1877 and then modified following the discovery of UCMP 77270 (Wann Langston undated, Wann Langston Papers, Series III: Museum Notes 1968-2005, Box VPA001/ 26, Texas Vertebrate Paleontology Archives; The Tuba City Dinosaur, The Samuel P. Welles Papers, Series 2: Dinosaur Files, University of California Museum of Paleontology Archives). The pubis comprises only the distal two-thirds of each bone (Fig. 19). The proximal ends of the ischia are present and coossified anteriorly to one another.

The left femur is complete, but the right femur only preserves the distal half, and its lateral condyle is broken
(Fig. 20). The tibiae are mostly complete, but both are missing their distal medial surfaces where they articulate with the astragalus (Fig. 21). The right fibula is present and the left fibula is incomplete distally (Fig. 22.1-22.7). The left astragalus and calcaneum are complete (Fig. 23). Those elements are separate but adhered to one another with plaster. The right astragalus is broken medially and most of the ascending process is missing. The right calcaneum is separate and complete. Distal tarsal 4 is preserved from both sides, and distal tarsal 3 is present from one side (Fig. 24). The left metatarsus is complete, as are metatarsals I, III, IV, and V on the right side. The distal end of right metatarsal I is preserved. Both pedes are complete except for the phalanges of digit I (Fig. 25).

UCMP 37303 (paratype specimen).-Locality UCMP V4214, Moa Ave 1, Coconino County, Navajo Nation, Arizona. See locality information for UCMP 37302 (Fig. 1). Welles (1984) described both premaxillae, and historical photographs confirm the existence of the right element, but it has not been with the rest of the specimen since at least 2010 (R. Tykoski, personal communication, 2014). The left premaxilla is complete and articulates with the left maxilla (Fig. 26). Both maxillae are present, but missing their posterior ends, and their dorsal margins along the antorbital fenestra and articulation with the nasals are incomplete. The anterior tip of the left maxilla is broken. The left palatine is crushed against the left maxilla, but an incomplete right palatine is preserved in isolation. A partial left ectopterygoid was found with a handwritten note and assigned to UCMP 37302, but Welles (1984) described this as the paratype specimen and, indeed, the fossil has " 37303 " written on it. Regardless, this element does not resemble a theropod ectopterygoid and is not included in this description. Two fragments of the nasolacrimal crest are present, but isolated, and the dorsal body of the right lacrimal is preserved. The dentaries are both present; the left dentary is mostly complete, but the right is missing its posterior margin (Fig. 27). The left splenial lies in articulation with the left dentary and the right splenial is isolated. The anterior third of the right splenial is missing. The basioccipital is present in isolation.

Vertebrae belonging to the paratype specimen are few and highly fragmentary (Fig. 28). Welles (1984) identified the posterior and anterior halves of cervical vertebrae 5 and 6 , respectively, presumably based upon those vertebrae from the holotype. We have repaired these vertebrae and they articulate with one another. The front portion of the centrum is present in presacral vertebrae 7 and 8 . Two trunk vertebrae are present, but are missing parts of each neural arch. The shape of the ventral half of the neural arch and position of the parapophyses are comparable to presacral vertebrae 19 and 20 in the holotype. The neurocentral sutures are open anteriorly and posteriorly, but the suture is obliterated in the middle of each vertebra. Fragments of trunk ribs are present. Vertebrae that compare favorably to the dorsosacral vertebra (vertebra 25) and caudosacral vertebra (vertebra 28) of the holotype are present in the paratype, but their transverse processes are broken off at their bases, the zygapophyses are missing, and the neural spines are broken. The first two caudal vertebrae are represented by their centra and neural arches, but every process is missing. The neural arch-centrum suture is completely closed and only visible as a slightly rugose patch of bone on the centrum of vertebra 30. Fragments of three more distal caudal vertebrae are present and their neural 

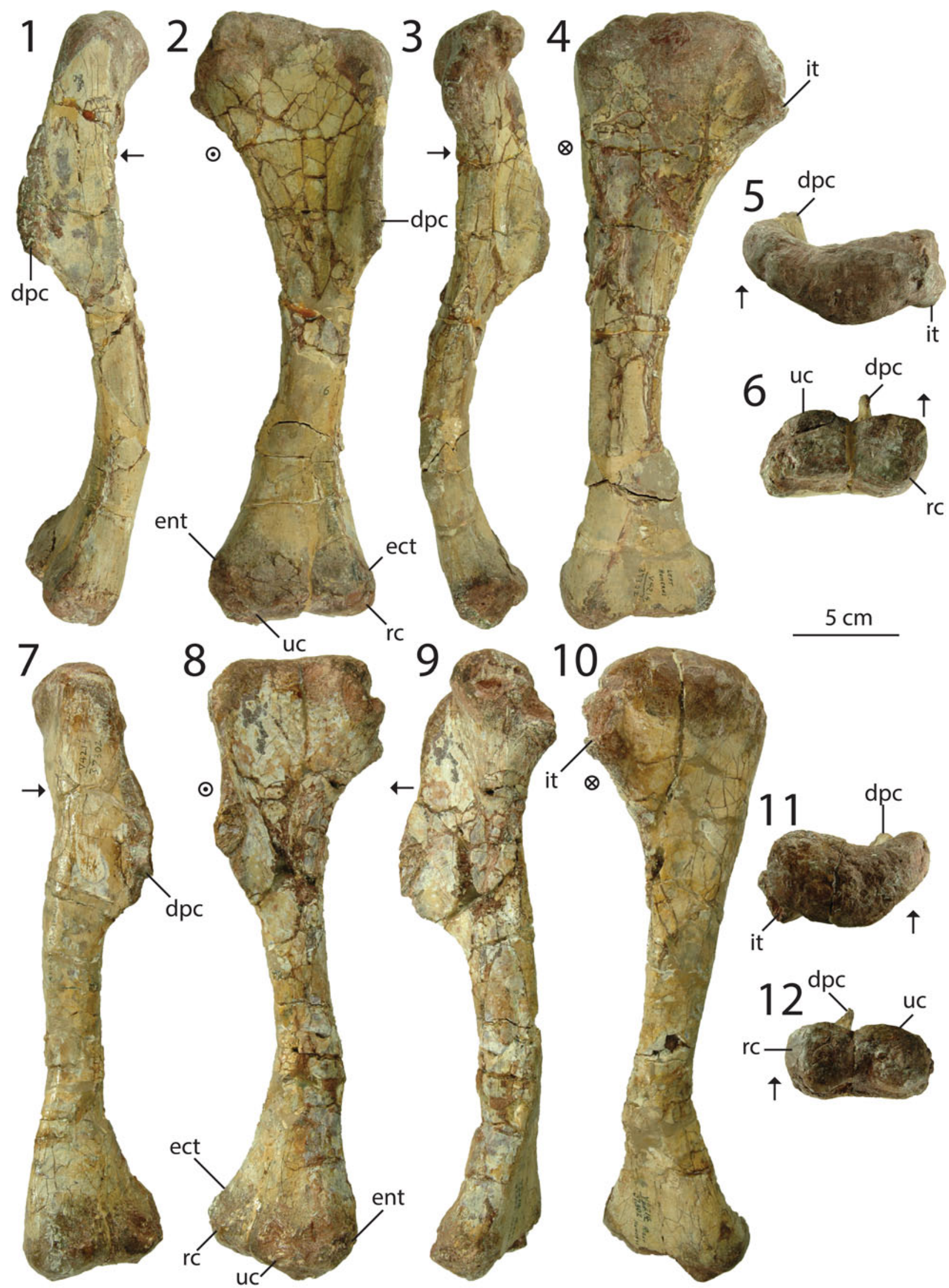

Figure 14. Dilophosaurus wetherilli holotype specimen (UCMP 37302): (1-6) right humerus and (7-12) left humerus in $(\mathbf{1}, \mathbf{7})$ lateral, $(\mathbf{2}, \mathbf{8})$ anterior, $(\mathbf{3}, \mathbf{9})$ medial, $(4,10)$ posterior, $(\mathbf{5}, 11)$ proximal, and $(\mathbf{6}, \mathbf{1 2})$ distal view. Arrows point in anterior direction. $\mathrm{dpc}=$ deltopectoral crest; ect $=$ ectepicondyle; ent $=$ entepicondyle; it $=$ internal tuberosity; $\mathrm{rc}=$ radial condyle; $\mathrm{uc}=$ ulnar condyle. 

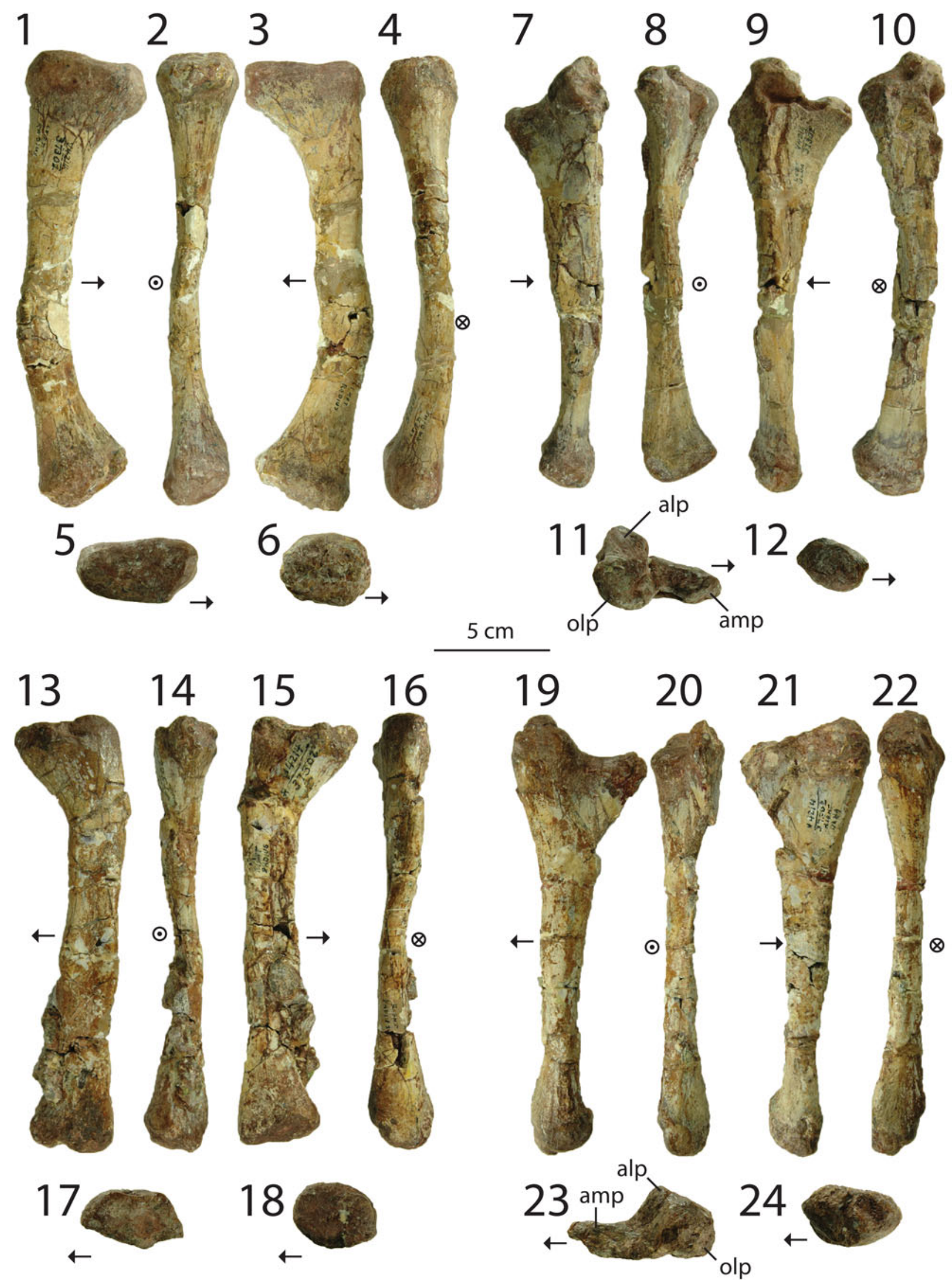

Figure 15. Dilophosaurus wetherilli holotype specimen (UCMP 37302): (1-6) left radius, (7-12) left ulna, (13-18) right radius, and (19-24) right ulna in (1, 7, 13, 19) lateral, $(\mathbf{2}, \mathbf{8}, \mathbf{1 4}, \mathbf{2 0})$ anterior, $(3,9,15,21)$ medial, $(4,10,16,22)$ posterior, $(\mathbf{5}, \mathbf{1 1}, \mathbf{1 7}, \mathbf{2 3})$ proximal, and $(\mathbf{6}, \mathbf{1 2}, \mathbf{1 8}, \mathbf{2 4})$ distal view. Arrows point in anterior direction. alp $=$ anterolateral process; amp $=$ anteromedial process; olp $=$ olecranon process. 
arches and centra are completely coossified to one another. The right scapula is badly broken and only preserves the glenoid region of the ventral end and part of the more dorsal scapular blade (Fig. 29.1, 29.2).

The glenoid region of the coracoid is also present and coossified to the ventral margin of the scapula. The distal end of the left humerus is present (Fig. 30.1-30.5), as are the worn proximal ends of both ulnae. The right ulna preserves more of the shaft. The left metacarpus is articulated; the first two metacarpals are complete, but metacarpals III and IV are missing their distal ends (Fig. 31.1-31.4). A small carpal is attached to the proximal end of the metacarpus and phalanx I- 1 is incomplete but articulated with metacarpal I. Metacarpals I and III are preserved from the left manus, but the first metacarpal is badly weathered. The distal ends of two non-terminal manual phalanges are present, as is an ungual from the first manual digit that is missing its distal tip.

The pelvic girdle is virtually absent from this individual; worn fragments may represent a proximal ischium and shaft. The distal end of the right ischium is present, but worn (Fig. 32.1-32.7). The distal ends of the left tibia and fibula, astragalus, and calcaneum are articulated but not coossified to one another (Fig. 33.1, 33.2). The tibia and astragalus are broken anteromedially (Fig. 34.1-34.4). The proximal end of metatarsal IV, distal end of right metatarsal III, and proximal end of the left metatarsal III are all that represent the pes (Fig. 35.1).

UCMP 77270.-Locality UCMP V6468, Moa Ave 2, Coconino County, Navajo Nation, Arizona, near the famous Tuba City dinosaur tracks south of the town of Moenave, AZ (Fig. 1), reportedly from the same stratigraphic horizon as the hypodigm (Welles, 1970, 1984). The right side of the skull of this specimen was preserved facing down and, as such, the left side has almost completely weathered away along the midline of most of the skull (Fig. 36). The 1964 specimen is larger and many of the bones in the skull are coossified to one another, and in some cases the sutures between them have been obliterated. The nasal and lacrimal are coossified into a mostly complete but warped nasolacrimal dorsal crest, and the maxilla has also coossified with the anterior nasal, but the suture is visible medially. The maxilla is complete and is coossified with the anterior portion of the jugal, which is all that remains of that bone. The right prefrontal is articulated with the lacrimal and frontal.

Both frontals and parietals are present, and the dorsal process of the left laterosphenoid is articulated with the bottom of the skull roof. The right squamosal is incomplete, but articulated with the right parietal and right paroccipital process of the braincase. A right quadrate is also present, but incomplete. The braincase is shattered in some places and was removed from the back of the skull during excavation or preparation (Fig. 37). The bones in the braincase are completely coossified and lack visible sutures with the exception of where the otooccipitals form part of the occipital condyle. The right palatine is stuck to the medial side of the jugal process of the lacrimal and the right pterygoid is incomplete except where it contacts the quadrate.

Both mandibles are well represented (Fig. 38). The articulars are complete and coossified to their corresponding surangulars and prearticulars. With the exception of the articulars, all of the bones in the posterior region of the mandibles are incomplete anteriorly, making articulation with the dentaries impossible to examine. Both dentaries are preserved, but are broken posteriorly. The right splenial is articulated and broken posteriorly and the left splenial is incomplete and isolated.

Consistent with the rest of the specimen, some of the vertebral column is beautifully preserved while other parts of it are glued-together, weathered piles of bone debris. The first four cervical vertebrae are preserved in articulation, but their right sides are weathered away (Fig. 39). The atlantal pleurocentrum and axial intercentrum are coossified to the front of the axial pleurocentrum, but the atlantal intercentrum is missing and was presumably not coossified to this complex. The rest of the bones of the neck are fairly well preserved. However, every vertebra is missing a zygapophysis, transverse process, neural spine, or some combination of these (Fig. 39). The neural arches are coossified to their centra and the sutures no longer are visible. The last cervical vertebra represented is probably the last vertebra in the neck (presacral vertebra 14). Welles estimated vertebral numbers, which he recorded on their respective collection cards as well as on the bones themselves. But after presacral vertebra ten, they almost never match and some bones have multiple numbers written on them. Field notes do not indicate any articulation in this region (Field Notes 1964-1968, The Samuel P. Welles Papers, Series 5: Field Notes, Box 10-11, University of California Museum of Paleontology Archives). Because the numbers have not been used in a publication, we disregard those numbers and have identified vertebral number by comparison to the holotype. The same problem is found throughout the trunk vertebrae, but the number and shape of each vertebra is consistent with the holotype. The historic numbering used by Welles for the trunk vertebrae during the curation of the specimen starts at thirteen, but the first trunk vertebra is more consistent with that of the holotype and is actually vertebra 15 (Fig. 40). Adding two to each historic number allows for 24 presacral vertebrae (consistent with the holotype) and confirms that vertebra 24 was not included as a dorsosacral into the pelvis. The dorsal and right lateral sides are missing from presacral vertebra 15 and 16 , and vertebra $17,18,19$, and 21 are badly weathered neural arches. Vertebra 20 comprises the neural arch and worn centrum. The centra of vertebrae 22 and 23 are coossified to one another; the neural arch is fragmentary on the former but complete on the latter. The transverse processes and neural spine are missing from vertebra 24 . The neural arches seem to all be coossified to their centra, but unlike the cervical vertebrae, this suture is visible throughout the trunk region.

The sacral series is not complete. Owing to the missing vertebrae around the base of the neck, the number of presacral vertebrae cannot be determined with certainty. Two centra and half of another are preserved in articulation with each other and the fragmentary right ilium; the two complete centra correspond to the caudosacral and first primordial sacral vertebra of the holotype (Fig. 40). The left sides of each neural arch are broken off, but articulated with and coossified to the left ilium. Comparisons to the holotype justify the presence of four vertebrae in the sacral series. The tail is highly fragmentary in this specimen; it consists of the back end of one anterior caudal vertebra and an anterior caudal centrum along with fragmentary mid-caudal vertebrae. The caudal neural arches and centra all seem to be coossified, and the isolated centra and arches present are probably broken rather than disarticulated. 
Incomplete cervical ribs are found articulated with the axis and third presacral vertebra. The tubercula of most of the cervical ribs are coossified to the diapophyses, but the capitula and parapophyses are generally unfused. Cervical ribs from presacral vertebrae 6-9 are present; the tubercula are broken off and usually remain on the vertebrae. Except for the ribs on the right side of vertebrae 6 and 8 , the shafts are broken distally. A single rib head is present from posterior cervical vertebrae. Broken shafts and fragmentary heads represent the thoracic vertebrae. Thin gastralia are present, but isolated and broken. One anterior haemal arch is present, as well.

The forelimb and pectoral girdle are poorly represented except for a coossified left scapulocoracoid (Fig. 29.6-29.9) and right radius and ulna (Fig. 41). The scapulocoracoid is missing the anterior margin of its dorsal blade. The distal end of a humerus is present, but poorly preserved. The distal end of the ulna is missing its lateral side.

Both ilia are present, but incomplete and crushed (Fig. 18.8-18.11). The three sacral vertebrae preserved have transverse processes that are coossified to the medial side of the iliac blades (Fig. 40.1-40.6). The ilia are missing their postacetabular processes, but parts of the preacetabular processes are present, especially on the left side. Although the suture is visible medially, the left proximal end of the left pubis is coossified to the left pubic peduncle of the ilium but does not preserve the obturator foramen or the distal end. The proximal end of the right ischium is broken along its contact with the ilium, but includes the antitrochanter (Fig. 32.8-32.12). The distal ends of both ischia are articulated, but the left is crushed. The left and right femora are present; the right is crushed proximally and is missing the crista tibiofibularis and the left is missing most of its proximal and distal ends (Fig. 42). The right tibia and fibula are complete (Figs. 22.8, 22.9, 33.3-33.8). The pes is represented by complete right metatarsals I-III, left metatarsal $\mathrm{V}$, distal left metatarsal III, and a proximal pedal phalanx either from digit II or III (Fig. 35.2-35.6).

Texas Vertebrate Paleontology Collections, The University of Texas at Austin, Austin, TX.-The three specimens housed in trust from the Navajo Nation in the Texas Vertebrate Paleontology Collections were collected from 1997 to 2000 by Timothy Rowe and field crews from the University of Texas at Austin. This material comes from the Adeii Eechi Cliffs on Ward Terrace, Arizona.

TMM 43646-1.-Locality TMM 43646, Dilophosaurus Quarry, Coconino County, Navajo Nation, Arizona in the 'middle third' of the Silty Facies of the Kayenta Formation near Gold Spring, AZ, type locality of Sarahsaurus aurifontanalis Rowe et al. (2011; Fig. 1). Referred to as the Type Sarahsaurus Quarry (Marsh, 2014), Rowe and a small group of graduate students found and excavated this quarry between 1997 and 2000. A partially articulated Dilophosaurus wetherilli was collected initially and the holotype and paratype specimens of Sarahsaurus aurifontanalis were found directly underneath. Preliminary laser ablation ICP-MS detrital zircon U-Pb results indicate a date of $183.7 \pm 2.7 \mathrm{Ma}$ (Early Jurassic, Pliensbachian; Marsh et al., 2014).

The skull and mandibles of this individual are represented by a variety of disarticulated but associated elements, including the isolated basioccipital, supraoccipital, parabasisphenoid, laterosphenoid, right prootic, left squamosal, left frontal, right quadratojugal, left jugal, left maxilla, right lacrimal, left articular, left splenial, left surangular and both laterosphenoids, postorbitals, pterygoids, quadrates, and dentaries (Figs. 43-45).

One atlantal neurapophysis and the pleurocentrum of the axis are preserved (Fig. 46). Most of the vertebrae preserved in this specimen are represented by isolated neural arches and centra, so we refrain from referring to their serial numbers. The cervical series includes two centra and three neural arches (Fig. 46). One fragmentary cervical rib is preserved. The trunk series includes isolated centra, two and four articulated centra, and isolated neural arches (Fig. 47). Various disarticulated and fragmentary trunk ribs are preserved. The sacral series is represented only by a sacral rib that corresponds best to the anteroposteriorly flared rib of the caudosacral in the holotype (Fig. 47). The tail is fairly complete, but it is impossible to identify the serial number of the disarticulated elements (Fig. 47). The caudal neural arches are disarticulated from their respective centra until around the last quarter of the tail. Three pieces of articulated caudal vertebrae are preserved from the very distal portion of the caudal series. No haemal arches were recovered.

The pectoral girdle and forelimb of this specimen comprise the left scapula, both coracoids (Fig. 29.10-29.18), partial left and right humeri (Fig. 30.6-30.11), metacarpal I, II, and III, and various manual phalanges, including one ungual (Fig. 48).

Both ilia are complete (Fig. 49.1-49.7). The right pubis only lacks its distal extremity, and the pubic apron and the ischia are represented by a fragment of the proximal end of the right element and coossified distal ends (Fig. 50). The right hindlimb was found in articulation and is complete (Figs. 22.12-22.15, 51-53), including a patella-like ossification preserved at the knee joint. The left hindlimb is more fragmentary and disarticulated (Figs. 22.12-22.15, 52, 53); the femur lacks most of the proximal and distal ends, the distal end is missing from the tibia, and the fibula, astragalus, calcaneum, and pes are complete (Figs. 34.5-34.10, 54).

TMM 47006-1.-Locality TMM 47006, Jon's Theropod, Coconino County, Navajo Nation, Arizona in the 'middle third' of the Silty Facies of the Kayenta Formation near Gold Spring, AZ (Fig. 1). This specimen preserves a complete braincase, including the parabasisphenoid, basioccipital, prootics, otooccipitals, supraoccipital, and laterosphenoids (Figs. 55-58), the left parietal, and the entire presacral vertebral column (except the atlas), some of which are in articulation (Figs. 59, 60).

TMM 43691-1 (cf. Dilophosaurus wetherilli).—Locality TMM 43691, Paiute North 1, Coconino County, Navajo Nation, Arizona, in the 'candy-stripe layer' of the Silty Facies of the Kayenta Formation near Paiute Canyon, AZ (Fig. 1). The specimen is one complete right ilium (Fig. 49.7, 49.8).

Museum of Northern Arizona, Flagstaff, AZ.-Several specimens at MNA have been referred to Dilophosaurus (Gay, 2001). However, in the absence of diagnostic apomorphies (Bell et al., 2004, 2010; Nesbitt and Stocker 2008), we refer some of them to "cf. Dilophosaurus wetherilli." They are too large to presently refer to Syntarsus kayentakatae and are within the size range of Dilophosaurus. MNA archives record that most of the bones from MNA 219-0 probably represent 


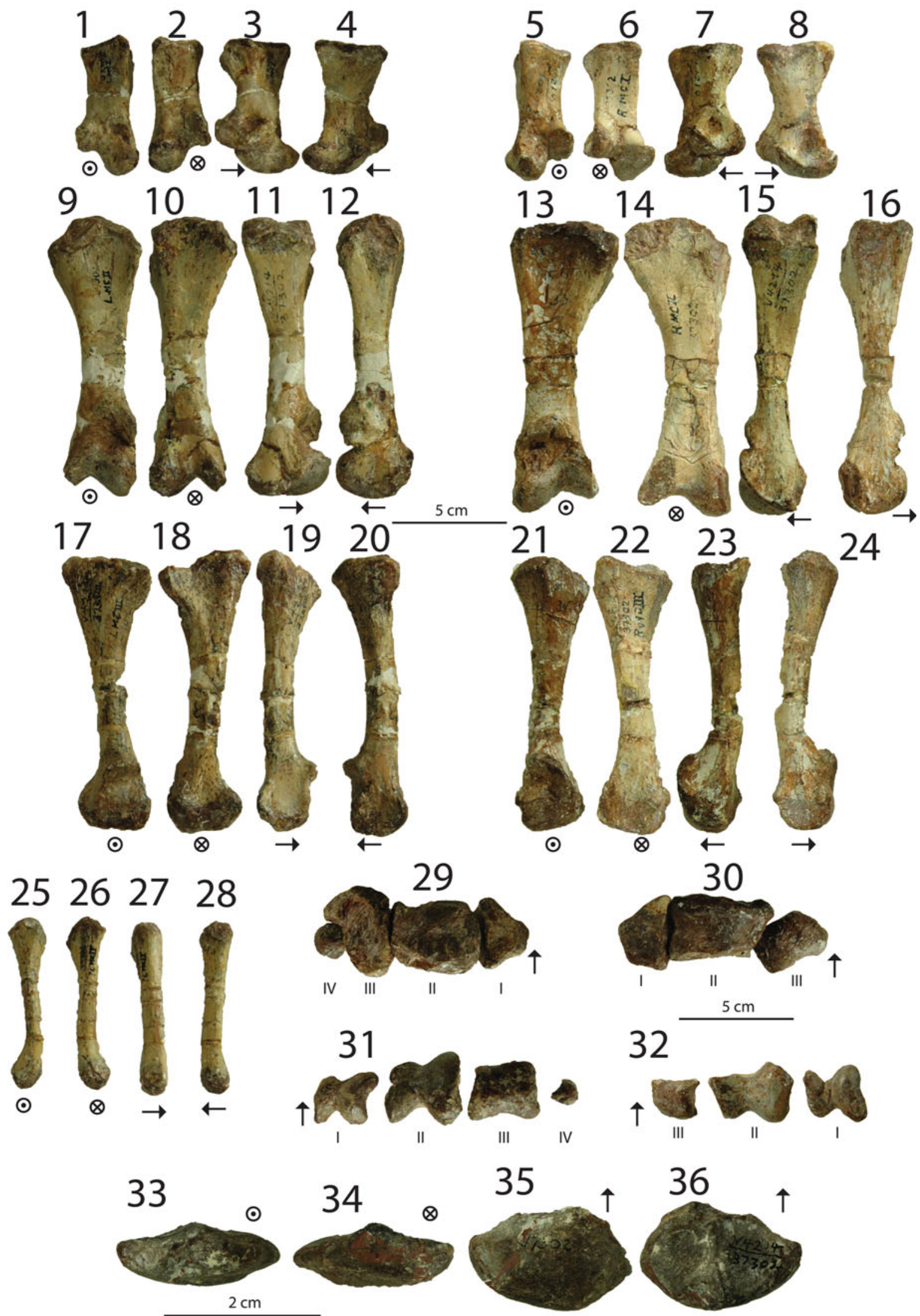

Figure 16. Dilophosaurus wetherilli holotype specimen (UCMP 37302): (1-4, 29, 31) left metacarpal I, (9-12, 29, 31) left metacarpal II, (17-20, 29, 31) left metacarpal III, (25-29, 31) left metacarpal IV, (5-8, 30, 32) right metacarpal I, (13-16, 30, 32) right metacarpal II, $(\mathbf{2 1 - 2 4}, \mathbf{3 0}, \mathbf{3 2})$ right metacarpal III, and (33-36) isolated carpal in $(1,5,9,13,17,21,25,33)$ dorsal, $(2,6,10,14,18,22,26,34)$ ventral, $(3,7,11,15,19,23,27)$ lateral, and $(4,8,12,16,20,24,28)$ medial view, $(\mathbf{2 9}, \mathbf{3 1}, \mathbf{3 5})$ proximal view, and $(\mathbf{3 1}, \mathbf{3 2}, \mathbf{3 6})$ distal view. Arrows point in dorsal direction. 
the fragmentary remains of a single individual, with the exception of MNA V3181 (the distal end of a coelophysoid pubis), MNA V109 (a complete ornithischian femur), and MNA V111, MNA V138, MNA V247, MNA V102, and MNA V122 (elements incongruent in size with the Dilophosaurus remains and attributable to Archosauria). The MNA specimens were collected by a joint crew from the Museum of Comparative Zoology at Harvard University and Timothy Rowe while working for MNA in 1978.

MNA V97, MNA V101, MNA V131, MNA V135, MNA V154, MNA V160/V161, MNA V176, MNA V177, MNA V248, MNA V530, MNA V539 (cf. Dilophosaurus wetherilli).-Locality MNA 219-0, Rock Head (Bowl Area), Coconino County, Arizona, Silty Facies of the Kayenta Formation near Rock Head, AZ (Fig. 1). Tooth, proximal end of right tibia, pedal phalanx, partial cervical vertebra, proximal end of left pubis, right femur, trunk centrum, caudal vertebra, proximal end of left tibia, proximal end of left fibula, proximal end of right fibula, respectively (Fig. 61.1-61.18).

MNA V3145 (cf. Dilophosaurus wetherilli).-Locality MNA 356-0, Gold Spring E (formerly Blue Valley), Coconino County, Arizona, Silty Facies of the Kayenta Formation near Gold Spring, AZ (Fig. 1). Distal end of right femur (Fig. 61.19-61.21).

Arches National Park, UT.-A highly fragmentary specimen (ARCH 4012) of a large dinosaur was collected from the Kayenta Formation in 2011 during a paleontological resource inventory at Arches National Park (DeBlieux et al., 2012; Madsen et al., 2012, fig. 11). The locality ARCH 71v occurs in the sandy Typical Facies of the Kayenta Formation near the Garden of Eden in the park and represents some of the only body fossils from that unit in Utah. The specimen includes fragments of vertebrae, limbs, and metapodials, and was thought to represent a theropod that "may be attributable" (Madsen et al., 2012, p. 17) to Dilophosarurus, but after reviewing the specimen, we cannot assign it to that genus using apomorphies, although its size is comparable to that of Dilophosaurus wetherilli.

\section{Description}

We describe each element under the assumption that all the specimens mentioned above belong to the type and only species of the genus, Dilophosaurus wetherilli. This assumption is tested in phylogenetic analyses below using each specimen as an operational taxonomic unit. Differences among these specimens are noted where relevant.

\section{Skull}

Premaxilla.-The premaxilla comprises a bulbous anterior body, long nasal process, and short maxillary process that forms most of the anterior and dorsal margin of the external naris, which circumscribes a subhorizontal ellipse (Figs. 26.1-26.4, 36). The narial fossa is not well developed in Dilophosaurus wetherilli, in contrast to sauropodomorphs (Sues et al., 2004; Prieto-Márquez and Norell, 2011; Sereno et al., 2012), Herrerasaurus ischigualastensis Reig, 1963 (Sereno and Novas, 1994), coelophysoids (Raath, 1977; Colbert, 1989; Rowe,
1989), and early averostrans (Madsen, 1976; Madsen and Welles, 2000). The lateral surface of the premaxilla anterior to the base of the nasal process is penetrated by eight foramina; the largest of those that are visible laterally is in the middle of the bone and opens anteroventrally. In lateral view, the dorsal margin of the bone is gently convex except for a subtle concavity where the nasal process meets the larger body of the bone (Fig. 26.1). The bottom half of the medial surface of the nasal process articulates with the nasal process from the other side at the midline. Above this articular surface, a dorsolateral convex ridge rises from the bone, and its smooth medial side indicates that it did not articulate along the midline. Instead, the thickened dorsolateral ridge on the nasal process of the premaxilla is continuous with the front margin of the nasolacrimal crest along the top of the skull. The nasal articulates with the nasal process of the premaxilla where a subelliptical depression and more posterior triangular groove open posteromedially on the distal tip of the nasal process.

The last two of the four alveoli still house teeth in UCMP 37303 , and the third tooth is much larger than the fourth. The premaxillary teeth are labiolingually compressed and have mesial and distal carinae. Serrations extend two-thirds up the distal carinae and the serrations on the mesial carina are faint, but extend halfway up the tooth. The medial surface of the premaxilla of UCMP 77270 is shattered and reveals replacement teeth about to erupt into the second and third of the four premaxillary alveoli. The first tooth is present in its socket and is serrated mesially, but not distally. The interdental plates are not coossified medially.

The maxillary process extends posteriorly from the alveloar process of the premaxilla, immediately behind the fourth alveolus (Fig. 36.2). Its lower lateral ridge is inset but continuous with the more prominent labial margin above the alveoli. A fossa indents into the ventral surface of the lateral maxillary process. It faces ventrolaterally, contributing to half of the subnarial gap between the premaxilla and maxilla. Medially, the premaxilla is mostly flat where it articulates with its counterpart. The lateral surface above the teeth has three large foramina. In medial view, a fourth foramen opens posteriorly in the anterior corner of the external naris. From it passes a groove that extends down the anteroventral rim of the naris into the posterior half of the maxillary process. The maxillary process of the premaxilla is concave medially, and its ventral margin is sharp and continuous with the labial margin above the tooth row. The medial concavity of the maxillary process receives the lateral side of the anteromedial (premaxillary) process of the maxilla (Figs. 26.2, 36.2). In lateral view, the maxillary process of the premaxilla covers the anteromedial process of the maxilla forming a roof over the subnarial gap. A foramen exits posteriorly on this surface. Another deep foramen opens in front of the anterior corner of the external naris. A groove emanates from it and extends parallel to the bottom of the narial opening.

The subnarial gap was originally conceptualized as a ligamentous articulation in Dilophosaurus wetherilli (Welles, 1984). This is one of the characters used to unite Dilophosaurus with coelophysoids (Welles, 1984; Rowe and Gauthier, 1990; Tykoski and Rowe, 2004). Owing to the close articulation of the premaxillae to one another and the robust, interlocking 

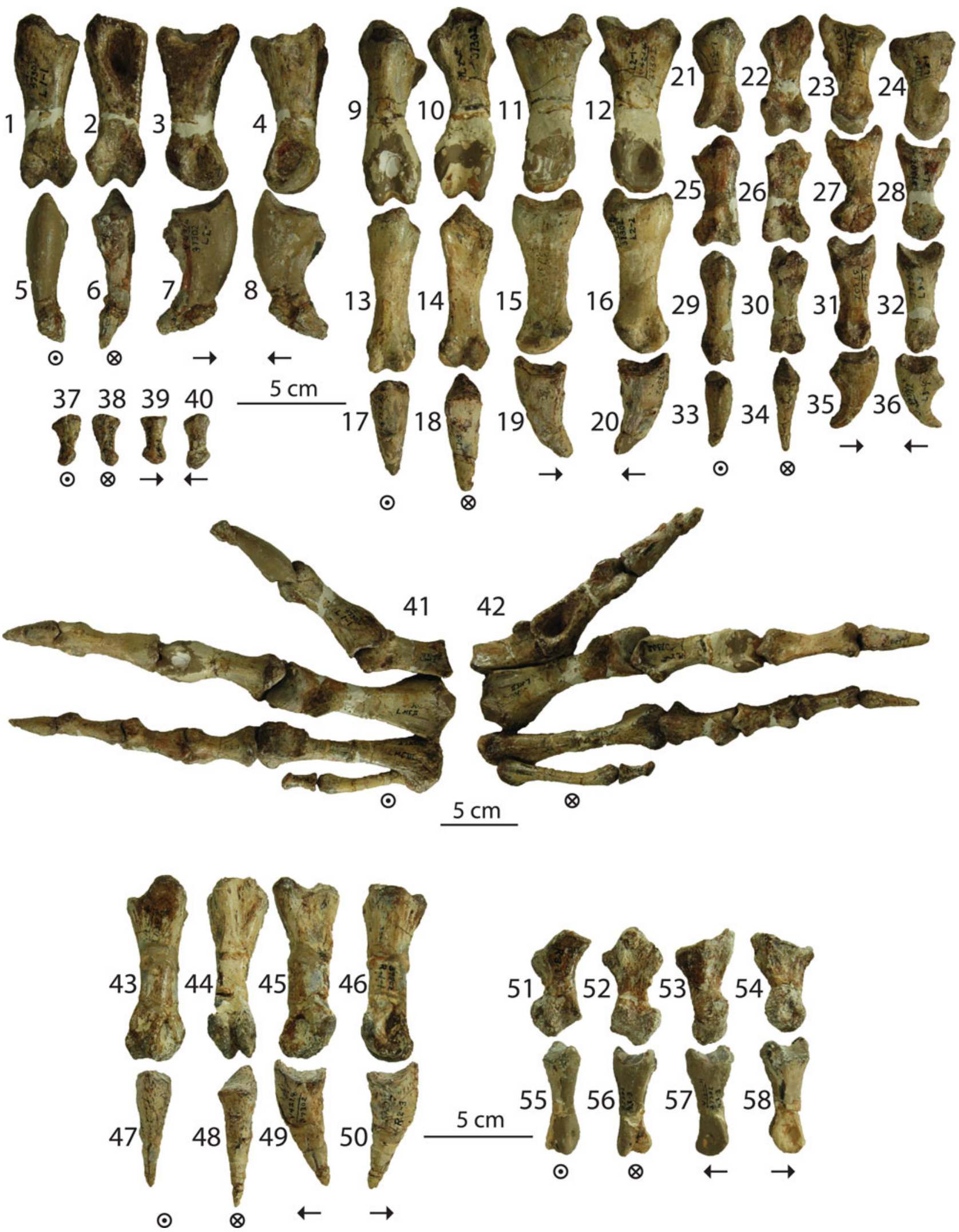

Figure 17. Dilophosaurus wetherilli holotype specimen (UCMP 37302): (1-4) left manual phalanx I-1, (5-8) left manual phalanx I-2, (9-12) left manual phalanx II-1, (13-16) left manual phalanx II-2, (17-20) left manual phalanx II-3, (21-24) left manual phalanx III-1, (25-28) left manual phalanx III-2, (29-32) left manual phalanx III-3, (33-36) left manual phalanx III-4, (37-40) left manual phalanx IV-1, (41, 42) articulated left manus, (43-46) right manual phalanx II-2, (47-50) right manual phalanx II-3, (51-54) right manual phalanx III-2, (55-58) right manual phalanx III-3 in $(\mathbf{1}, \mathbf{5}, \mathbf{9}, \mathbf{1 3}, \mathbf{1 7}, \mathbf{2 1}, \mathbf{2 5}, \mathbf{2 9}, \mathbf{3 3}, \mathbf{3 7}, \mathbf{4 1}, \mathbf{4 3}, \mathbf{4 7}, \mathbf{5 1}, \mathbf{5 5})$ dorsal, $(\mathbf{2}, \mathbf{6}, \mathbf{1 0}$, $14,18,22,26,30,34,38,42,44,48,52,56)$ ventral, $(3,7,11,15,19,23,27,31,35,39,45,49,53,57)$ lateral, and $(4,8,12,16,20,24,28,32,36,40,46,50,54,58)$ medial view. Arrows point in dorsal direction. 
articulation of the anteromedial process of the maxilla to the maxillary process of the premaxilla, this joint was strong and immobile. We note that the subnarial gap is now known to be present in other dinosaurs, including coelophysoids (Raath, 1977; Colbert, 1989; Rowe, 1989), Tawa hallae (Nesbitt et al., 2009b), Eoraptor lunensis Sereno et al., 1993 (Sereno et al., 2012), and some heterodontosaurids (Sereno, 2012). In the latter taxon, the gap accommodates a large lower caniniform tooth and may not be homologous to the subnarial gap of saurischians. In either case, the subnarial gap is now considered apomorphic at the level of Theropoda or possibly a more inclusive taxon.

Maxilla.-Only UCMP 77270 is complete enough to preserve the entire maxillary tooth row (Fig. 36.1-36.4). The right maxilla houses fourteen alveoli, but only the ninth still contains a tooth, albiet shattered. The labiolingually widest and mesiodistally longest alveoli are the third and fourth. The anteriormost four teeth project anteroventrally. The last maxillary tooth lines up beneath the jugal process of the lacrimal. This is unlike the condition found in the sauropodomorphs Plateosaurus engelhardti von Meyer, 1837 and Massospondylus carinatus Owen, 1854 and in coelophysoids, where the tooth row continues behind the ventral extent of the lacrimal (Rowe, 1989; Prieto-Márquez and Norell, 2011).

The holotype right maxilla includes the posteriormost six alveoli (Fig. 3.1-3.4). Complete teeth are found in the last, third-to-last, and anterior-most alveolus. Lacking the complete maxilla, the tooth count in the holotype specimen is estimated to be 13-14. Anteriorly, the teeth increase in length and all have serrated mesial and distal carinae.

The left and right maxillae are preserved in UCMP 37303 and include 12 and 10 alveoli, respectively (Fig. 26.1-26.5). Both are incomplete posteriorly, where they articulated with the jugals. The first alveolus of both maxillae is empty, but its shape indicates that the first tooth projected anteroroventrally. The anterior margin of the maxilla is convex. Behind the gap, the alveolar margin of the maxilla becomes horizontal. The second, third, and fourth alveoli gradually reorient from anteroventrally to ventrally directed. The longest teeth in the left maxilla occupy the third and fourth alveoli, but these may have slipped out of their sockets. The maxillary teeth are strongly curved, especially towards the front of the mouth, and serrated mesial and distal carinae are present on all preserved maxillary teeth. A row of foramina penetrates the alveolar margin of the maxilla just above the base of the teeth, beginning just above maxillary tooth three. Behind the ninth tooth, these foramina coalesce into longer grooves.

Interdental plate anatomy of UCMP 37302 is unknown owing to the aggregation of bones in that area. In UCMP 37303 , the interdental plates are coossified lingually and between the alveoli except for a gap medial to the second tooth on the right maxilla and the ninth tooth on the left maxilla (Fig. 26.5). The interdental plates of UCMP 77270 are not coossified anteriorly lateral to the nutrient groove, but those between teeth six and eight are fused into a plate (contra Hendrickx and Mateus, 2014). Only a few interdental plates are preserved in TMM 43646-1, but they are distinct from one another (Fig. 43.2).

The maxilla and lacrimal of UCMP 77270 are coossified at their suture to form continuous surfaces medially and laterally
(Fig. 36.1, 36.2). The antorbital fossa is deepest anterolaterally behind a vertical lamina extending up the ascending process. A smaller subelliptical fossa is present in the anteroventral corner of the antorbital fossa. The promaxillary foramen is well developed, lies just above the smaller depression, and is mostly hidden by the anterior lamina bounding the front of the antorbital fossa. Blind pockets, like those found in Syntarsus kayentakatae and Ceratosaurus nasicornis Marsh, 1884 (we refer all Morrison Formation specimens of this genus to the type species sensu Rauhut, 2003; Carrano and Sampson, 2008; Malafaia et al., 2014), are found in the anteroventral corner of the antorbital fossa (Rowe, 1989; Madsen and Welles, 2000). The ventral margin of the promaxillary foramen is incised into the anterior surface of the remaining portion of the ascending process. The dorsal margin of the antorbital fenestra is smooth behind the ascending process and tapers posteriorly. The lateral side of the maxilla is smooth in Dilophosaurus wetherilli (Figs. 3.1, 26.1, 26.3, 36.1, 43.1), Daemonosaurus chauliodus Sues et al., 2011, and early averostrans (Nesbitt, 2011). The lateral surface of the maxilla lacks the longitudinal alveolar ridge that delimits the ventral margin of the antorbital fossa of Eodromaeus murphi Martínez et al., 2011, Liliensternus liliensterni (von Huene, 1934), coelophysoids (Raath, 1977; Colbert, 1989; Rowe, 1989), Zupaysaurus rougieri (Ezcurra, 2006), and Cryolophosaurus ellioti (Smith et al., 2007). In the articulated skull of UCMP 77270, the bottom of the antorbital fenestra is demarcated by a lamina of bone that is continuous with the rest of the lateral side of the maxilla, but is inset relative to the tapering jugal process above the tooth row.

A groove for the articulation of the nasal extends up the dorsal surface of the maxilla, starting between the anteromedial process and main body of the maxilla and terminating at the ascending process. The anterodorsal groove houses one to two larger foramina (depending on the specimen) near the ascending process, which itself contains the promaxillary sinus that communicates externally through the promaxillary foramen (Witmer, 1997). The base of the ascending process is anteroposteriorly long and extends between the second and sixth teeth (Figs. 26.1, 36.2). Its dorsal edge articulates and is confluent with the nasal, and the long posterodorsal process is thin above the antorbital fenestra. In dorsal view, the anterior end of the maxilla is convex laterally and the posterior end is convex medially; the inflection point occurs between the seventh and eighth maxillary teeth. Like that of coelophysoids (Raath, 1977; Colbert, 1989; Rowe, 1989), the angle formed by the horizontal body of the maxilla and the ascending process is $<35^{\circ}$. This is unlike the condition in Herrerasaurus ischigualastensis (Sereno and Novas, 1994), Tawa hallae (Nesbitt et al., 2009b), Zupaysaurus rougieri (Ezcurra, 2006), and early averostrans (Madsen, 1976; Bonaparte, 1986; Madsen and Welles, 2000), as well as the maxilla tentatively referred to Lepidus praecisio Nesbitt and Ezcurra, 2015, in which the angle is between $35^{\circ}$ and $50^{\circ}$.

The anteromedial process (Figs. 26.2, 26.4, 36.2, 43.2) is a prominent pointed process that is much longer than that of early averostrans including Allosaurus fragilis (Madsen, 1976) and Piatnitzkysaurus floresi Bonaparte, 1979 (Bonaparte, 1986) and is similar to that of Sinosaurus triassicus (Xing, 2012). It has a concave lateral surface that is obscured by the medial 

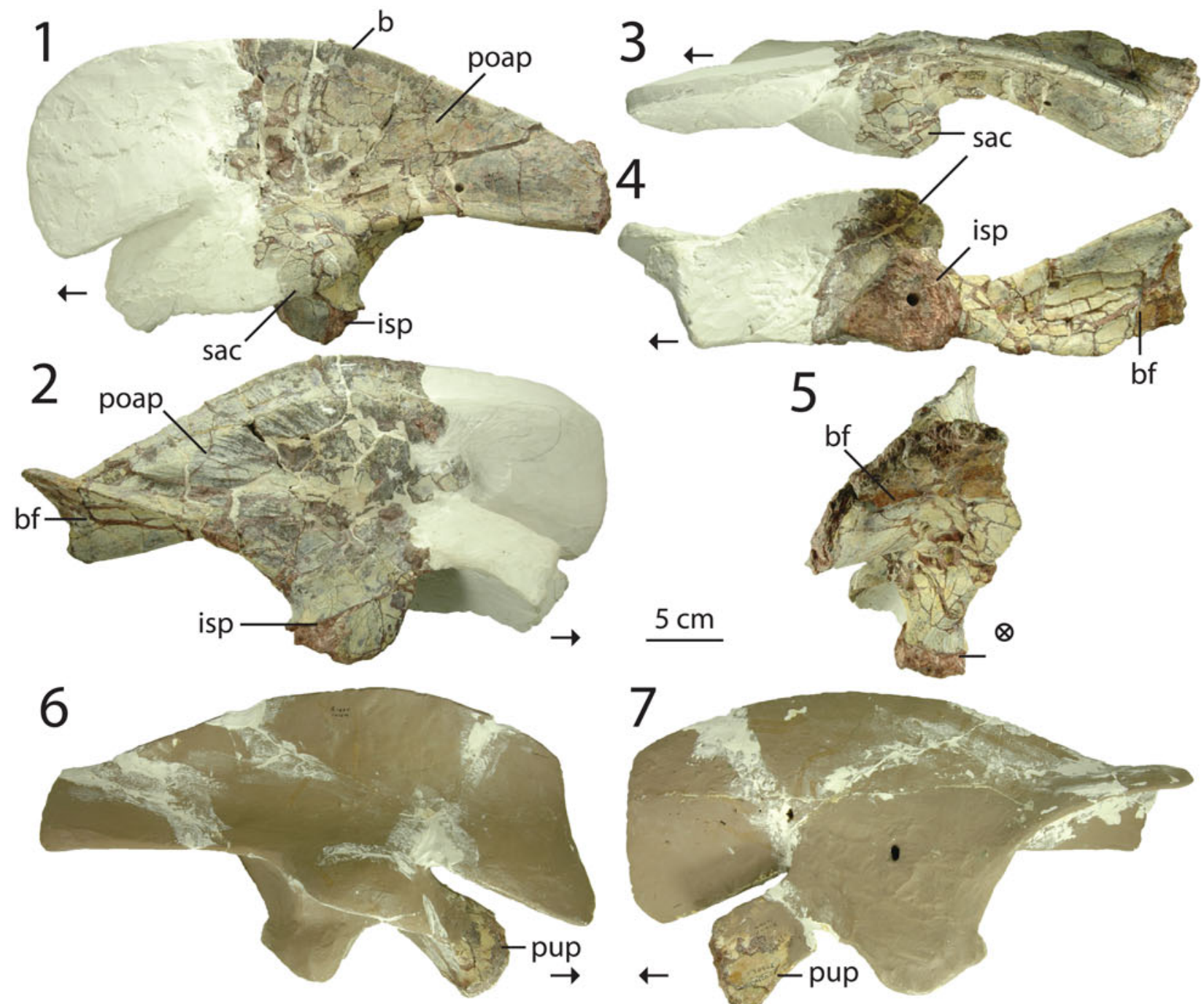

$\otimes$

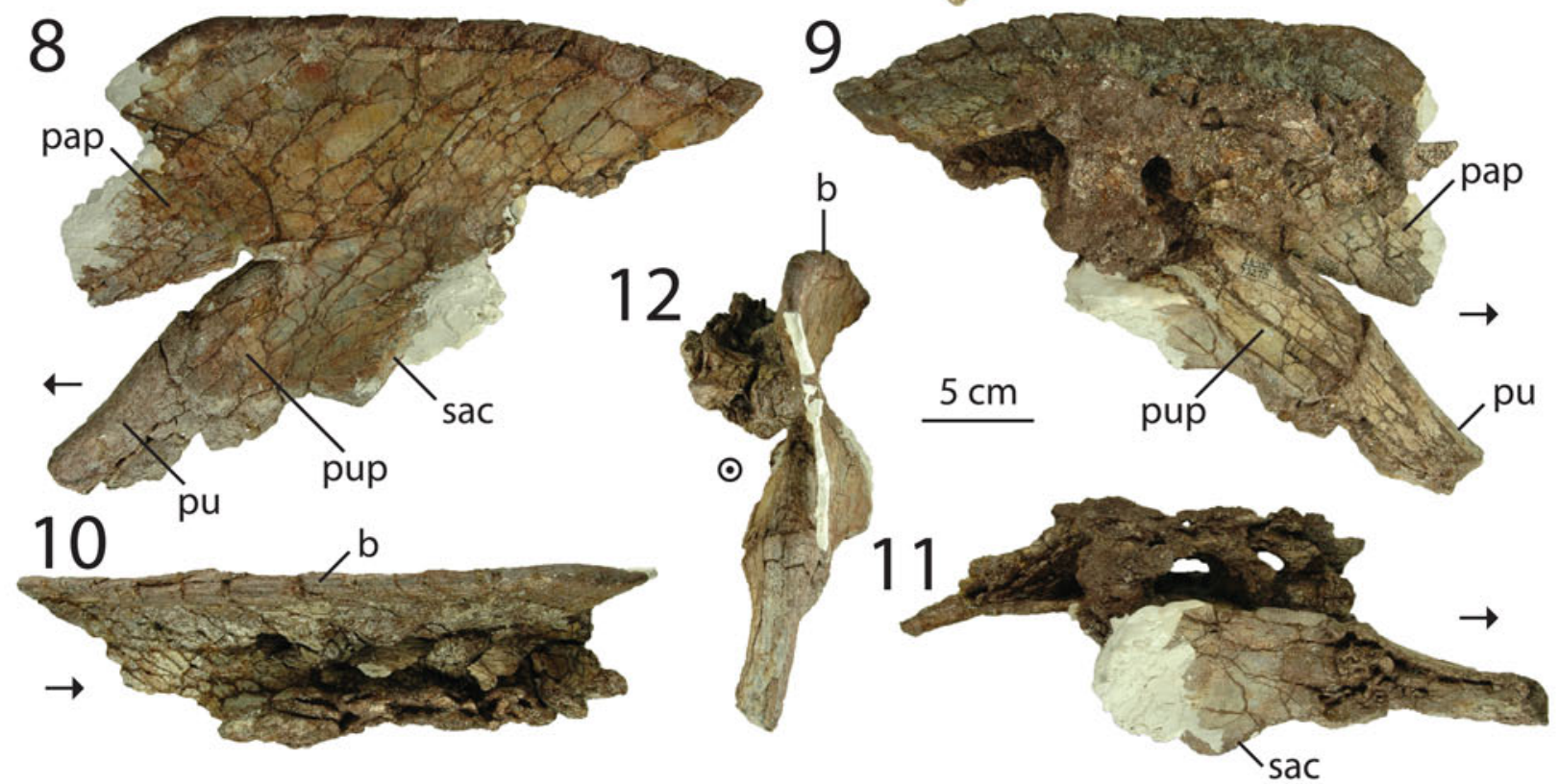

Figure 18. Dilophosaurus wetherilli holotype specimen (UCMP 37302): (1-5) left ilium and (6,7) right ilium; Dilophosaurus wetherilli referred specimen (UCMP 77270): (8-12) left ilium and pubis. $(\mathbf{1}, \mathbf{6}, \mathbf{8})$ Lateral, $(\mathbf{2}, \mathbf{7}, \mathbf{9})$ medial, $(\mathbf{3}, \mathbf{1 0})$ dorsal, $(\mathbf{4 , 1 1})$ ventral, $(\mathbf{5})$ posterior, and $(\mathbf{1 2})$ anterior view. Arrows point in anterior direction. $\mathrm{b}=$ blade; $\mathrm{bf}=$ brevis fossa; isp = ischial peduncle; $\mathrm{pap}=$ preacetabular process; poap = postacetabular process; pu = pubis; pup = pubic peduncle; sac = supraacetabular crest. 
surface of the maxillary process of the premaxilla at the subnarial gap. Two horizontal grooves pass along the medial surface of the anteromedial process and follow its curvature; the dorsal groove is longer and deeper than the ventral groove. The anteromedial processes of the left and right maxilla of UCMP 37303 articulate medially along their longitudinal grooves. A long nutrient groove extending along the lingual surface of the medial side of both maxillae has two foramina deeply incised dorsal to the second and third teeth of the right maxilla and the first and second teeth of the left side. The medial side of the jugal process of the maxilla is too obscured or broken to see any articular surface for the palatine.

Nasal and lacrimal.- The nasals and lacrimals form the characteristic pair of cranial crests in Dilophosaurus (Figs. 3.1-3.6, 3.9, 3.10, 26.6-26.10, 36, 43.9, 43.10, 43.15, 43.16). The nasal and lacrimal are coossified and the suture between them is impossible to determine. Isolated fragments of the crest are preserved in UCMP 37302, UCMP 37303, and TMM 43646-1. The dorsal margin of the antorbital fossa is continuous with the surface of the nasolacrimal crest in articulated specimens (UCMP 37302 and UCMP 77270; Figs. 3.1, 36.1). UCMP 77270 is the only specimen to preserve the anterior part of the nasal. The element is bifurcated anteriorly where it forms the dorsal and posterior margins of the external naris, but the bone is too broken here to estimate the entire shape of the naris. A thin sheet of bone wraps down laterally to articulate with the front of the ascending process of the maxilla, and the lateral surface is continuous with the lateral surface of the nasolacrimal crest. A horizontal shelf extends medially towards the midline to contact the other nasal. The crests of Dilophosaurus wetherilli were almost certainly covered with keratin or keratinized skin, and a recent study on the cranial ontogeny of the extant guinea fowl Numida meleagris (Linnaeus, 1758) (Angst et al., 2019) suggests that the keratin on the crests of $D$. wetherilli could have been much more prominent than what the bony tissue indicates.

The lacrimal articulates with the medial side of the jugal in a shallow triangular fossa (Fig. 43.9) that narrows into a short anteriorly directed groove at the midpoint of the posterior margin of the antorbital fenestra. This is the same location where the lacrimal is at its thinnest anteroposterior width. In crosssection, the shaft of the lacrimal is elliptical. The lacrimal expands greatly into a preorbital boss and the nasolacrimal crest. The thick, rugose preorbital boss forms an arc outlining the anterodorsal margin of the orbit (Figs. 3.1, 3.2, 3.10, 26.6, $36.1,43.10)$. It is thickest anteriorly, projects slightly posteriorly into the orbit, and supports the bottom of the back of the crest. Preorbital bosses are found in Zupaysaurus rougieri (Ezcurra, 2006), Cryolophosaurus ellioti (Smith et al., 2007), early tetanurans, including Allosaurus fragilis (Madsen, 1976) and Ceratosaurus nasicornis (Madsen and Welles, 2000), and in the abelisaurs Carnotaurus sastrei Bonaparte, 1985 (Bonaparte et al., 1990) and Majungasaurus crenatissimus Depéret, 1896 (Sampson and Witmer, 2007). A triangular slot forms the articulation for the prefrontal in Dilophosaurus wetherilli. The prefrontal lies behind and beneath the rugose area of the lacrimal.

Anteriorly, the lacrimal thins considerably to form the dorsal margin of the antorbital fenestra and crest (Figs. 3.1, 26.10, 26.11, 36.1, 36.2). In posterior view, the lacrimal foramen penetrates the region where the ventral shaft of the lacrimal meets the postorbital process. A pair of thin ridges extends laterally to the lacrimal foramen and passes it ventrally. The thickened ridge of the lacrimal that forms the orbital rim continues posteriorly in UCMP 77270, where it is braced by the prefrontal, and forms a small process on the back end of the nasolacrimal crest. This process is subtriangular in posterior outline and the surface of unbroken bone on its dorsal edge is short, indicating that the rest of the crest rose dorsolaterally from the skull over a distance of $\sim 12 \mathrm{~mm}$. This posterior process of the crest curves slightly outward. The medial side of the orbital region of the lacrimal forms a pocket with the prefrontal and frontal behind the posterodorsal corner of the antorbital fenestra.

Isolated pieces of the nasolacrimal crest in UCMP 37303 preserve the thin dorsal body of the crest as well as a longitudinal medial shelf that reached the midline to touch the nasolacrimal on the other side (Fig. 26.8-26.10). With the medial shelf held horizontally in anteroposterior view, the crests rise from the skull roof at an angle of $\sim 80^{\circ}$. The crest of UCMP 77270 projects dorsolaterally from the skull roof, but its poor preservation makes it difficult to measure that angle or interpret the shape or texture of the medial and lateral surfaces. Where it is preserved, the nasolacrimal crest is tallest above the position of the eighth maxillary tooth, or approximately at the midpoint of the length of the antorbital fenestra. The skull roof is too crushed in UCMP 77270 to determine the nature of the contact between the frontal and nasal.

The nasolacrimal crest begins as a low ridge on the premaxilla of Dilophosaurus wetherilli (Fig. 36.1, 32.2) in contrast to the taller contribution found in Sinosaurus triassicus (Xing et al., 2015) and the bifurcated slotted articulation of Monolophosaurus jiangi Zhao and Currie, 1993 (Brusatte et al., 2010). The construction of the parasagittal crests of Shuangbaisaurus anlongbaoensis is difficult to assess, but seems to incorporate dorsal expansions of the frontals, which is unique among non-avian theropods (Wang et al., 2017). If a crest was indeed present on the nasals of Dravovenator regenti (Yates, 2005), it did not incorporate the nasal process of the premaxilla as does Dilophosaurus wetherilli. It is not clear whether the incipient crests reported in Dracovenator regenti (Yates, 2005) and Syntarsus kayentakatae (Rowe, 1989) are crushed nasal or lacrimal bones, such as the reported crest of Zupaysaurus rougieri was determined to be (Arcucci and Coria, 2003; Ezcurra, 2006; Ezcurra and Novas, 2007). Cryolophosaurus ellioti (Smith et al., 2007), Monolophosaurus jiangi (Brusatte et al., 2010), and oviraptorosaurs (Clark et al., 2002; Lamanna et al., 2014) are all theropods with a single midline crest. The crest of $C$. ellioti comprises only the nasal and lacrimal and is mediolaterally expanded. The crest of $M$. jiangi also includes the premaxilla, is highly pneumatic, and is anteroposteriorly expanded. The crest of oviraptorosaurs comprises primarily a dorsal expansion of the premaxillae with some contribution of the nasals. Other non-avian averostrans, including Ceratosaurus nasicornis (Madsen and Welles, 2000), Allosaurus fragilis (Madsen, 1976), some tyrannosaurids (Carr, 1999; Brusatte et al., 2012), and Caihong juji (Hu et al., 2018), exhibit lacrimal elaborations or 'horns.' Ceratosaurus nasicornus and Spinosaurus aegiptiacus Stromer, 1915 also have a midline crest that emanates from the nasals (Madsen and Welles, 2000; dal 

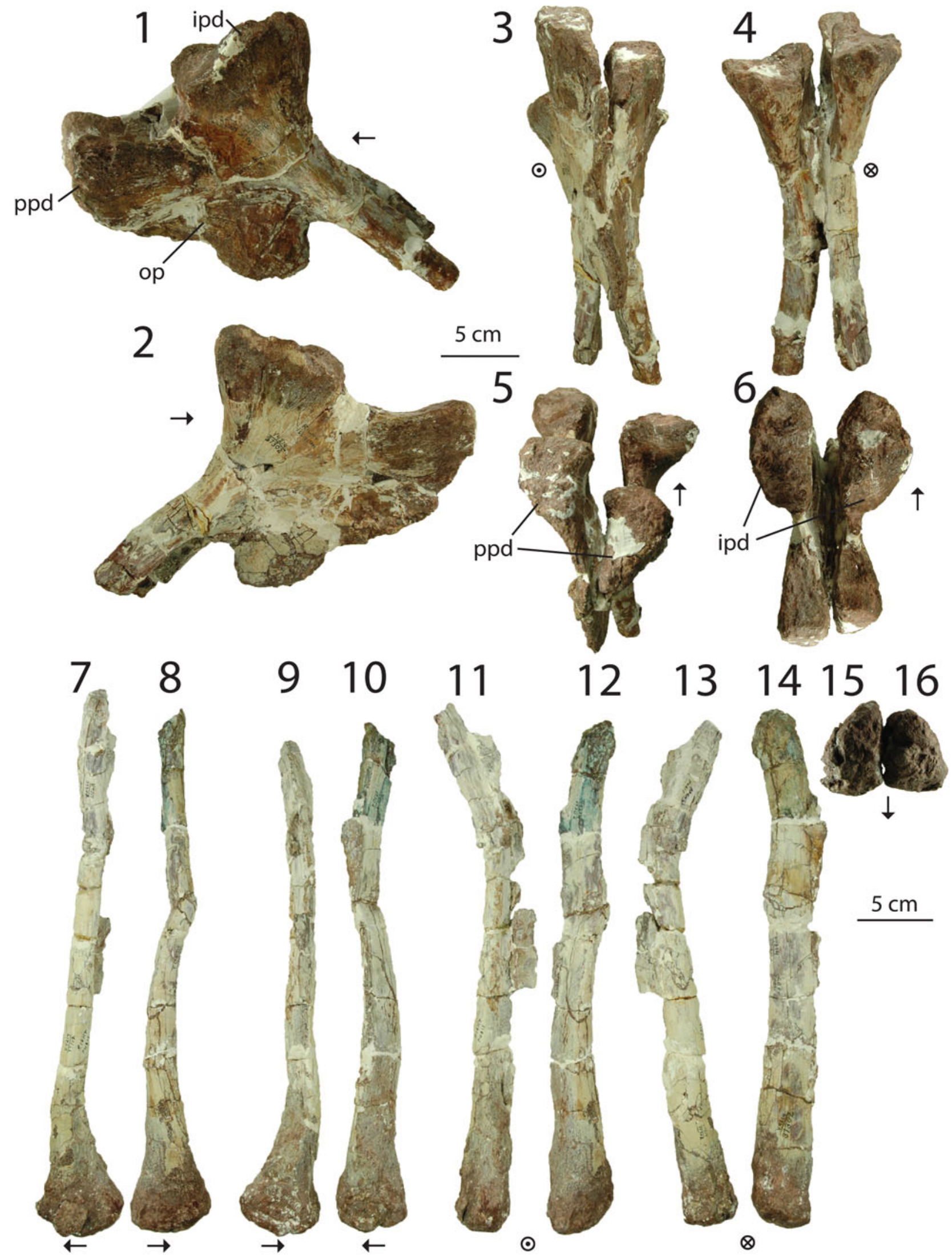

Figure 19. Dilophosaurus wetherilli holotype specimen (UCMP 37302): (1-6) left and right ischia, $(\mathbf{7 , 9}, \mathbf{1 1}, \mathbf{1 3}, \mathbf{1 5})$ left pubis, and $(\mathbf{8 , 1 0 , 1 2 , 1 4 , 1 6 )}$ right pubis in $(\mathbf{1}, \mathbf{7})$ left lateral, $(\mathbf{2}, \mathbf{8})$ right lateral, $(\mathbf{9})$ left medial, $(\mathbf{1 0})$ right medial, $(\mathbf{3}, \mathbf{1 1}, \mathbf{1 2})$ anterior, and $(\mathbf{4 , 1 3 , 1 4})$ posterior view. Other views are the $(\mathbf{5})$ proximal ends of the pubic pedicles of the ischia, $(\mathbf{6})$ proximal ends of the iliac pedicles of the ischia, and the $(\mathbf{1 5}, \mathbf{1 6})$ distal ends of the pubes. Arrows point in anterior direction. ipd $=$ iliac pedicle; op = obturator process; ppd = pubic pedicle. 
Sasso et al., 2005). Supraorbital horns of Carnotaurus sastrei are dorsolateral extensions of the frontals (Bonaparte et al., 1990).

Prefrontal.- The right prefrontal is best preserved in the holotype specimen and is closely articulated with the back of the lacrimal (Fig. 3.1-3.4). The prefrontal shares a thickened rugose texture with the lacrimal and forms the posterior end of the preorbital boss. Medially, the articulation between the prefrontal and lacrimal forms an open pocket that faces forward into the antorbital fenestra. The right prefrontal is also preserved in UCMP 77270 (Fig. 36). Ventrally, the surface of this bone forms a smooth anterodorsal corner of the orbit. The posterior process is visible ventrally where it slots into its corresponding groove on the ventrolateral surface of the frontal. The prefrontal does not contribute to the nasolacrimal crest.

Jugal.-The jugal is best preserved in UCMP 37302 (Fig. 3.1-3.4), and it is broken in TMM 43646-1 (Fig. 43.3, 43.4). It is anteroposteriorly long and bifurcates posteriorly where it meets the anterior process of the quadratojugal, as in other dinosaurs. The quadratojugal process of the jugal extends below the infratemporal fenestra and terminates near the back of the quadratojugal. The dorsal ramus of the jugal is only $\sim 40 \%$ of the length of the quadratojugal ramus. The jugal-quadratojugal articulation of Dilophosaurus wetherilli is similar to that in Tawa hallae (Nesbitt et al., 2009b), Liliensternus liliensterni (von Huene, 1934), Coelophysis bauri (Colbert, 1989), and Zupaysaurus rougieri (Ezcurra, 2006), where it lies below the posterior edge of the maxillary process of the jugal. This articulation is found well behind the maxillary process of the jugal in Cryolophosaurus ellioti (Smith et al., 2007), Sinosaurus triassicus (Xing, 2012), and averostrans such as Allosaurus fragilis (Madsen, 1976).

The maxillary process of the jugal rises slightly and overlaps the maxilla, where it contributes to the posteroventral corner of the antorbital fenestra at nearly a right angle (Figs. 3.1, 36.1). Two processes extend dorsally from the jugal; the shorter lacrimal process and long postorbital process form an elliptical margin to the bottom half of the orbit. The postorbital process is oriented posterodorsally towards the infratemporal fenestra, as in Eodromaeus murphi (Martínez et al., 2011) and coelophysoids (Raath, 1977; Colbert, 1989; Rowe, 1989). Ventrally, the jugal is gently convex with a slight concavity beneath the lacrimal process, where the maxilla is slotted into this bone. The lateral surface of the jugal is flat (Figs. 3.1, 43.3) and lacks a longitudinal ridge that occurs in Heterodontosaurus tucki Crompton and Charig, 1962 (Sereno, 2012), Eoraptor lunensis (Sereno et al., 2012), Herrerasaurus ischigualastensis (Sereno and Novas, 1994), Eodromaeus murphi (Martínez et al., 2011), Liliensternus liliensterni (von Huene, 1934), and coelophysoids (Raath, 1977; Colbert, 1989; Rowe, 1989). The right jugal of UCMP 77270 is coossified to the jugal process of the maxilla (Fig. 36.1, 36.2). The suture is almost obliterated. Photographs taken of UCMP 77270 between 1971 and 1972 show that the lacrimal process completed the posteroventral corner of the antorbital fenestra at a right angle. Unfortunately, it is now broken and cannot be repaired without removing the support struts built into the specimen.

Postorbital.-The ventral end of the postorbital expands into a flattened, triangular articular surface for the jugal (Figs.
3.1-3.4, 3.7, 3.8, 36, 43.1-43.14). A groove twists up the length of postorbital and forms a pocket in the back of the medial side of the bone, facing the top of the infratemporal fenestra. The postorbital shaft is triangular in cross-section. Laterally, the top of the postorbital forms another rugose, thickened area in the posterodorsal corner of the orbit. The anterior surface of the dorsal end of the postorbital is mostly flat, but a sharp edge is formed laterally with this surface and the lateral rugosity. Both postorbitals of the holotype have very shallow depressions behind their lateral rugose surfaces (Fig. 3.1, 3.7). The posterior process of the postorbital is sharply pointed and curves ventrally to slot into the front of the squamosal at the top of the infratemporal fenestra. A subcircular pit is found on the posterodorsal corner of the bone in medial view that receives a process from the dorsal process of the laterosphenoid (best seen in TMM 43646-1 and UCMP 77270).

Frontal.- The shape of the frontal of UCMP 77270 is difficult to determine because as many as five bones articulate with it (Figs. 36, 37.1). The contact with the nasal is not preserved in any specimen. A ventrolateral triangular groove for the prefrontal is present on the front half of the bone. A semicircular fossa on the ventral surface demarks the position of the olfactory bulbs (Fig. 43.7, 43.8). The frontal contributes to the dorsal margin of the orbit where it is bounded by the anterior articular facet for the prefrontal and the posterior postorbital process. In UCMP 77270, the broken left laterosphenoid covers the area where the frontal and parietal meet and there is no visible suture there. A midline suture is present between the frontals. The anterior margin of the supratemporal fossa is depressed into the rear dorsal surface of the frontal (Fig. 43.7). The anterior end of the frontal tapers anteriorly like that of Cryolophosaurus ellioti (Smith et al., 2007), some early ornithischians (Sereno, 1991, 2012), and most pseudosuchian archosaurs (Nesbitt, 2011).

Parietal.- The parietals are coossified to one another and to the frontals in UCMP 77270 (Figs. 36, 37.1, 37.2), which is the most skeletally mature specimen. A single, disarticulated parietal is present in the small specimen TMM 47006-1 (Fig. 58.13-58.18), another indication of its relative immaturity. The top of the skull roof of UCMP 77270 appears to be perforated by a fontanelle, but this is probably a result of breakage. A deep midline groove begins between the postorbital processes of the frontals and extends back to open into the top of the endocranial cavity. The parietals, supraoccipital, and portions of the braincase of UCMP 77270 are coossified, shattered, or covered with sufficient epoxy to obscure anatomical details. The lateral wall of the parietal borders the top of the supratemporal fenestra, where they are thin, and a posterior contribution by the parietal to the supratemporal fossa cannot be determined. By rearticulating the isolated parietal and braincase of TMM 47006-1, it can be determined that the parietals do not overlap the anterodorsal surface of the supraoccipital as they do in Cryolophosaurus ellioti (Smith et al., 2007). The posterior portions of the parietals meet along the midline in front of the supraoccipital forming a short, low ridge.

Squamosal.- The right squamosal of UCMP 37302 is broken ventrally and medially (Fig. 3.1-3.4). It articulates with the postorbital anteriorly where the postorbital process is indented by a groove into which the postorbital articulates. A short horizontal ridge extends from the posterodorsal corner of the 


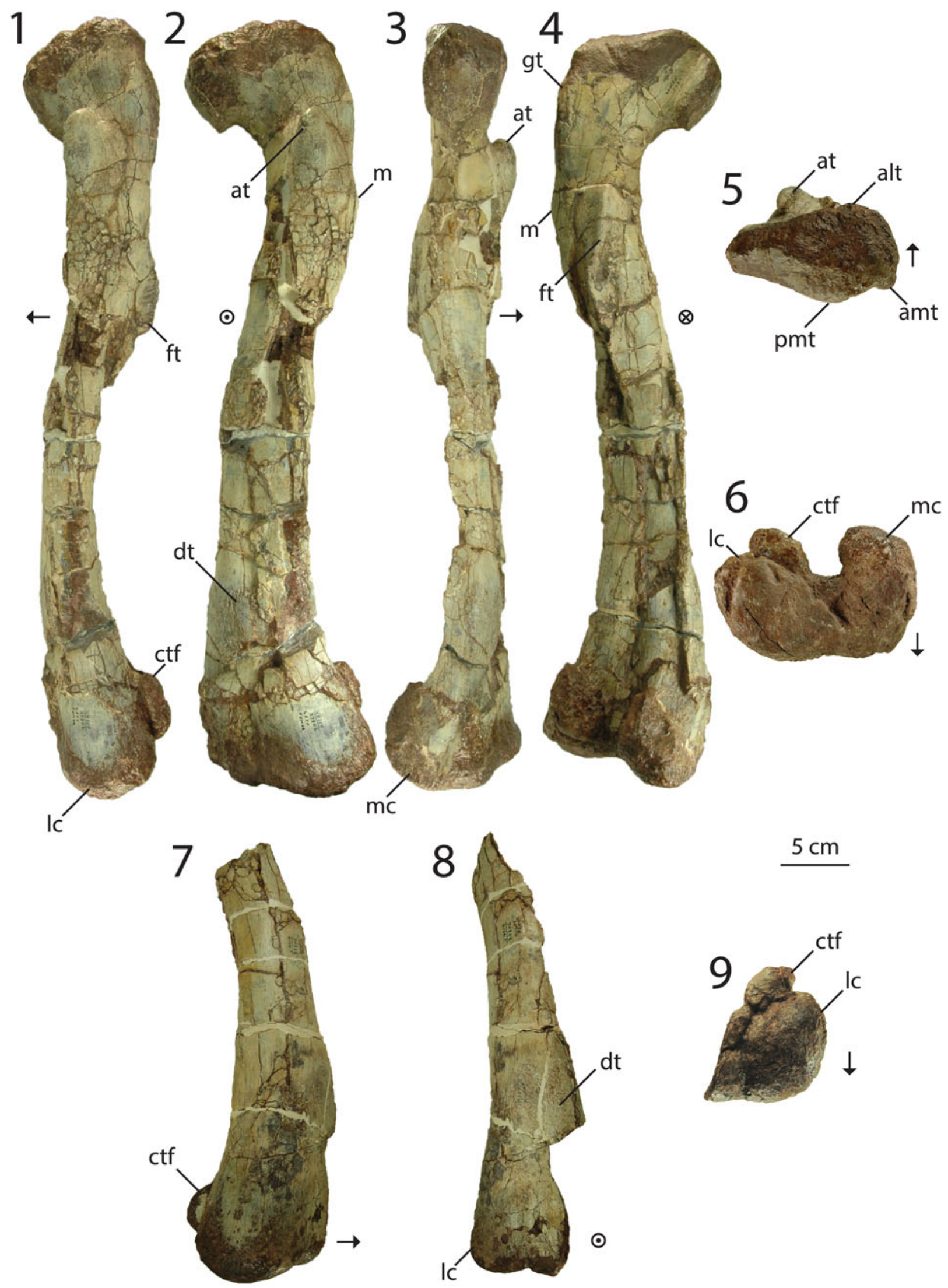

Figure 20. Dilophosaurus wetherilli holotype specimen (UCMP 37302): (1-6) left femur and (7-9) distal end of right femur in $(\mathbf{1 , 7})$ lateral, $(\mathbf{2 , 8})$ anterior, $(\mathbf{3})$ medial, $(\mathbf{4})$ posterior, $(\mathbf{5})$ proximal, and $(\mathbf{6}, \mathbf{9})$ distal view. Arrows point in anterior direction. alt $=$ anterolateral tuber; amt $=$ anteromedial tuber; at $=$ anterior trochanter; $\mathrm{ctf}=$ crista tibiofibularis; $\mathrm{dt}=$ distal tuberosity; $\mathrm{ft}=$ fourth trochanter; $\mathrm{gt}=$ greater trochanter; $\mathrm{lc}=$ lateral condyle; $\mathrm{m}=$ mound; $\mathrm{mc}=$ medial condyle; $\mathrm{pmt}=$ posteromedial tuber. 
infratemporal fenestra to the base of a paddle-like posterior process that lies lateral to the quadrate cotylus. Under that ridge is a fossa on the lateral side of the quadrate process that is deepest dorsally. In posterior view, the squamosal has a flattened top that is continuous all the way back to the tip of the posterior process. This process is inflected laterally and forms the ceiling of the quadrate cotylus. The quadrate process is very thin and tapers ventrally to barely contact the quadratojugal as in coelophysoids (Raath, 1977; Colbert, 1989; Rowe, 1989; Tykoski, 1998; Figs. 3.1, 3.2, 43.17). Medially, the squamosal has a deep recess at the intersection of the postorbital, quadrate, and posterior processes. That recess lies opposite to another on the medial surface of the postorbital that creates a larger infratemporal fossa on the medial surface of the skull. The articulation with the paroccipital process of the braincase is not preserved.

The right squamosal of UCMP 77270 is coossified with the posterior process of the parietal along its anteromedial margin (Fig. 36). The distal postorbital and quadrate processes are broken, but the posterior process is complete and in articulation with the paroccipital process of the braincase. A ridge extends from the back of the posterior process to the postorbital process along the top of the squamosal. In posterior view, the posterior process is subtriangular in outline and its dorsomedial face braces the flattened surface of the paroccipital process. The quadrate cotylus is broad and forms a smooth depression that receives the head of the quadrate (Fig. 43.18). In lateral view, the posterior end of the squamosal extends behind the head of the quadrate in a condition unlike that in Cryolophosaurus ellioti (Smith et al., 2007) and Zupaysaurus rougieri (Ezcurra, 2006) in which the posterior end of the squamosal does not extend posterior to the head of the quadrate.

Quadrate.-The body of the quadrate is tall (Figs. 5.11$5.20,36,43.20-43.31$ ). In posterior view, the body of the quadrate begins just above the lateral condyle and twists along its length up the back of the skull. It terminates at the posteromedial surface of the quadrate head. The posterior margin of the quadrate is sigmoid in medial view, where the head is offset posteriorly relative to the condyles. The strut is slightly thickened and raised near its dorsal terminus. The head is rounded with a small groove on the posterior margin where it articulates into the quadrate cotylus of the squamosal. It has a short anterolateral projection immediately below where the lateral flange begins. The pterygoid flange is triangular in medial view, and its dorsal margin is straight and inclined $\sim 15^{\circ}$ down from the horizontal. A fossa is located on the medial side of the pterygoid flange and is deepest just below the midpoint of the bone (Figs. 5.12, 5.18, 36.2, 43.21, 43.27). Below this point, the front margin of the pterygoid flange becomes continuous with the condylar region of the quadrate via an arching ridge that forms a small semicircular, ventrally concave fossa. The lateral flange is continuous farther down the quadrate and terminates at the same level as the lateral condyle, where it articulates as a roughened patch of bone with the quadratojugal. The pterygoid flange originates from the main body of the quadrate closer to the head than does the lateral flange, but the lateral flange is more continuous with the ventral condylar surface than the pterygoid flange. Most of the quadrate foramen is formed where the lateral flange is pinched in next to the medial side of the quadratojugal (Figs. $5.14,5.20,43.23)$. This configuration is similar to other early theropods; the quadrate foramen is lost in ceratosaurians, and it is a large opening between the quadrate and quadratojugal in avetheropodan tetanurans (Tykoski, 2005; Smith et al., 2007). Another fossa is found on the lateral side of the lateral flange dorsal to its contribution to the quadrate foramen. The anterior face of the lateral flange is visible ventrally. The condylar region of the quadrate contains medial and lateral condyles that are separated by a groove that extends anteromedially in ventral view. The medial condyle is more pronounced and has a small elliptical recess on its medial side.

Quadratojugal.-Most of the quadratojugal is flat (Figs. 3.1-3.4, 3.11, 3.12, 43.5, 43.6) except for the quadrate process, which twists dorsally along a sharp ridge behind the posteroventral corner of the infratemporal fenestra. The posterior surface of quadratojugal behind that ridge is continuous with the lateral flange of the quadrate when the two bones are articulated. A shallow groove extends down the ventral margin of the quadratojugal that articulates with the ventral arm of the quadratojugal process of the jugal. The corresponding jugal process of the quadratojugal extends forward to the anterior margin of the infratemporal fenestra.

Palatine and pterygoid.-The left palatine of the holotype is crushed up against the medial side of the right maxilla, but it is too crushed to identify anything more than the expanded dorsal process and a laterally facing longitudinal concavity behind the dorsal process (Fig. 3.2). The isolated right palatine of UCMP 37303 is incomplete and preserves the maxillary, jugal, and pterygoid processes, but these are broken distally (Fig. 27.8-27.11). The top margin of the dorsal process is broken where the palatines meet at the midline. The pterygoid process is visible in lateral view. Lateral and medial laminae stretch from the posterior edge of the dorsal process posteriorly and form a pocket between the dorsal process and jugal and pterygoid processes. Another smaller fossa is found posteriorly between the jugal and pterygoid processes. These fossae are separated from each other by an oblique lamina. The pterygoids of TMM 43646-1 (Fig. 45.13-45.16) are very similar in shape to those of Ceratosaurus nasicornis (Madsen and Welles, 2000).

\section{Braincase}

Basioccipital.-The sutures between the basioccipital and the otooccipital are visible and easily distinguishable where the preservation allows in all available specimens (Figs. 4, 26.11-26.13, 37, 44.13-44.18, 55, 58.49-58.54). The basiocciptal and parabasisphenoid can be distinguished from one another in all specimens except UCMP 77270, in which the suture between these elements is coossified and not visible externally. The occipital condyle is wider than it is tall. Sharp semicircular ridges extend along the lateral side of the basioccipital and are concave posteriorly. Each ridge points posteriorly along the ventral margin of the bone where it articulates with the parabasisphenoid at the basal tubera. Those ridges are where the posteroventral margin of the parabasisphenoid articulates with the basioccipital below the hypoglossal foramina. In posteroventral view, the basioccipital has two struts of bone that extend from the bottom of the occipital condyle to the basal tubera, similar to those found in Syntarsus rhodesiensis (Raath, 1977) and Murusraptor barrosaensis Coria and Currie, 2016 (Paulina-Carabajal and Currie, 2017) (Fig. 58.52). 


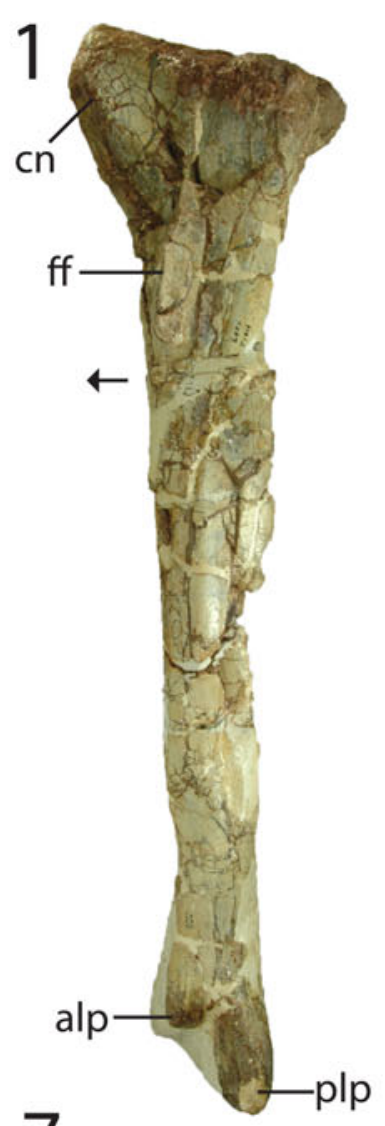

7

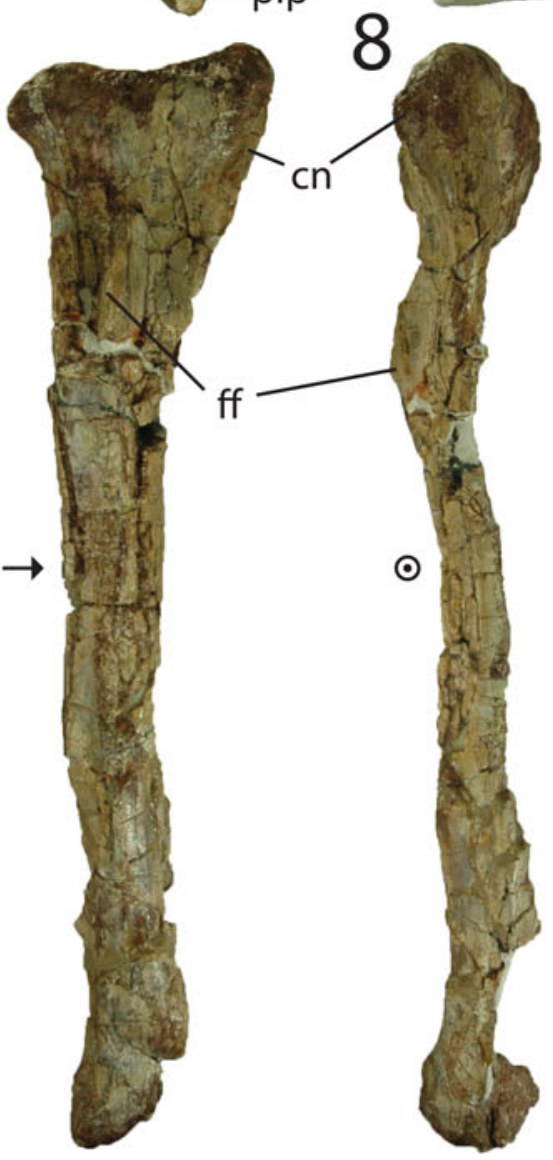

3

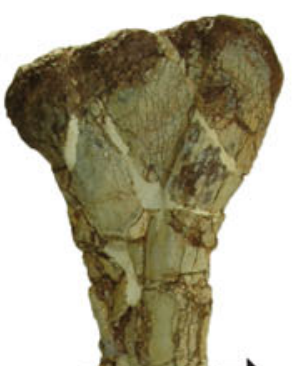

$\odot$

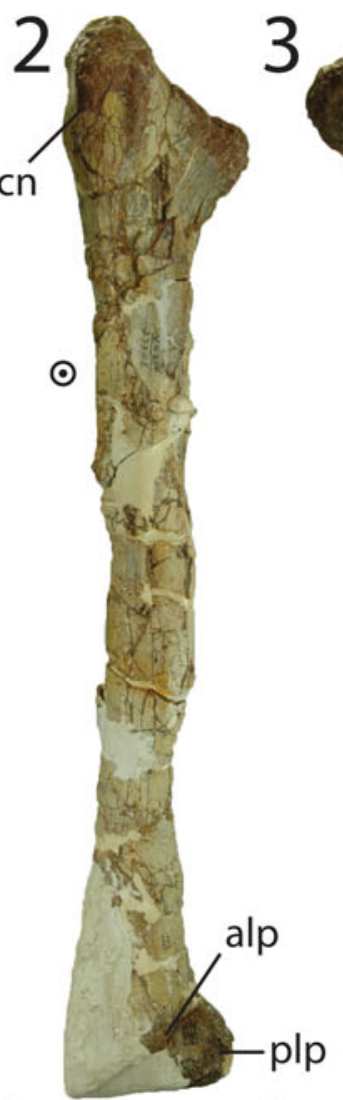

9

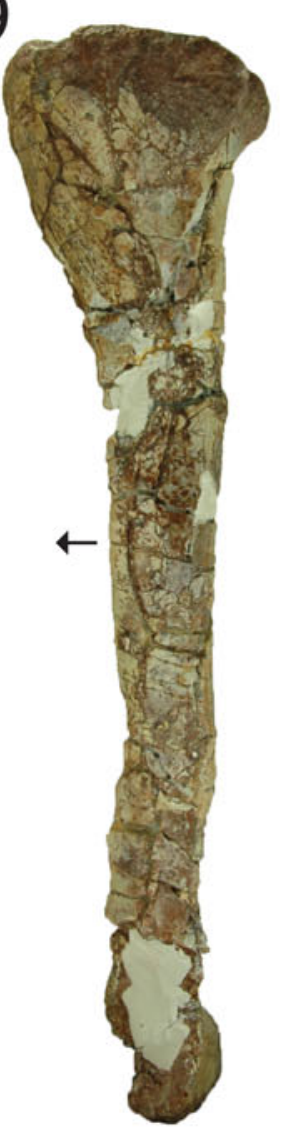

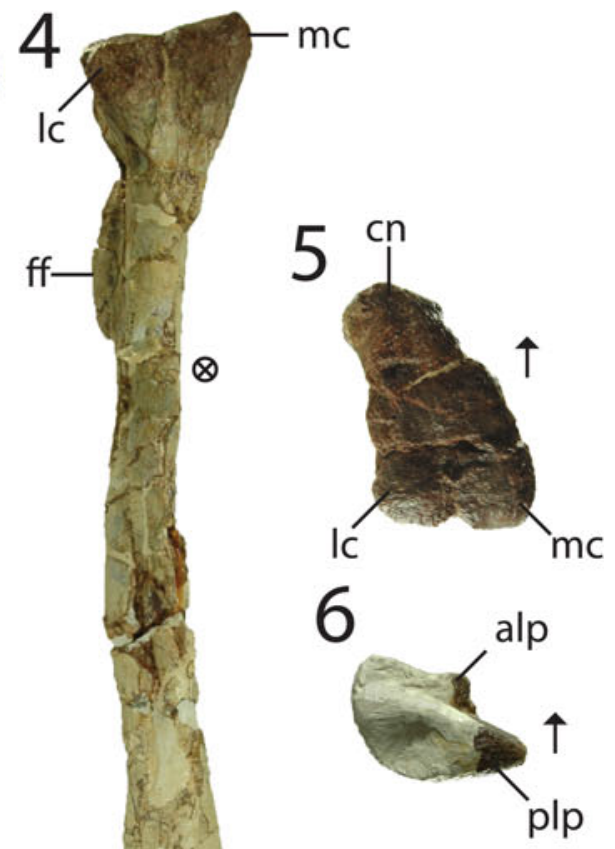

$5 \mathrm{~cm}$

Figure 21. Dilophosaurus wetherilli holotype specimen (UCMP 37302): (1-6) left tibia and (7-12) right tibia in (1, 7) lateral, $(\mathbf{2}, \mathbf{8})$ anterior, $(\mathbf{3 , 9})$ medial, $(\mathbf{4 ,}, \mathbf{1 0})$ posterior, $(\mathbf{5}, \mathbf{1 1})$ proximal, and $(\mathbf{6}, \mathbf{1 2})$ distal view. Arrows point in anterior direction. alp $=$ anterolateral process; $\mathrm{cn}=\mathrm{cnemial} \mathrm{crest} ; \mathrm{ff}=\mathrm{fibular}$ flange; lc $=$ lateral condyle; $\mathrm{mc}=$ medial condyle; $\mathrm{plp}=$ posterolateral process. 
A groove, the subcondylar recess, is bounded by those two struts. The basioccipital contribution to the basal tubera is subtriangular. A groove on the dorsal surface of the basiocciptal represents the most proximal portion of one of the hypoglossal foramina (CN XII) that exits the side of the otooccipital. The anteroventral portion of the basioccipital makes up much of the basisphenoidal recess, which lies between the basioccipital contribution to the basal tubera and the parabasisphenoid; TMM 47006-1 is the only specimen complete enough to preserve this feature (Figs. 56.4, 58.54). Two very deep pockets are excavated into the basisphenoidal recess, dividing it into bilateral halves, as in Syntarsus kayentakatae (Tykoski, 1998).

Parabasisphenoid.-As in many archosaurs, the basisphenoid and parasphenoid are coossified to one another to form the parabasisphenoid (Figs. 4, 37, 44.19-44.22, 55, 58.5558.60). This bone articulates with the basioccipital dorsally and has two medially directed platforms that support the basal tubera. Lateral to those platforms are thickened 'wings' of the posterodorsal margin of the parabasisphenoid, which are slightly rounded in lateral view. The ventral-most margin of the basisphenoidal recess is formed by the parabasisphenoid. The basipterygoid processes of the basisphenoid extend from the ventral part of the bone and diverge posteriorly. A transverse web of bone spans the proximal portion of each basipterygoid process under the ventral level of the parabasisphenoid 'wings' mentioned above. In TMM 47006-1, a small median process extends posteroventrally from this transverse web of bone (Fig. 56.1). A similar median process is present in Sinosaurus triassicus (Xing et al., 2014), but it houses a deep, wide pocket posteriorly. The basipterygoid processes expand distally and enclose an elliptical subsellar recess between them. The internal carotids enter the parabasisphenoid bilaterally beneath the articular surface for the posterior portion of the ventral process of the prootic. The tall, triangular pituitary (=hypophyseal) fossa is open anteriorly below the dorsum sellae. A pair of small foramina that allowed passage of the internal carotid arteries penetrate the posteroventral corner of the fossa. The cultriform process of the parasphenoid is not well preserved in any specimens except TMM 47006-1. Its cultriform process has a pair of bilateral basipterygoid recesses near its base, a midline subcultriform recess, and a dorsal cultriform groove.

Each lateral side of the basisphenoid component of the parabasisphenoid has three major pneumatic features. The sides of the parabasisphenoid have a caudal tympanic recess (preserved best in UCMP 77270 and TMM 47006-1) below the ascending process of the parasphenoid and anterolateral to the basal tubera (Witmer, 1997) (Figs. 37.3, 55.7). A sharp ridge inside this feature divides it into anterior and posterior halves. The subotic component of the rostral tympanic recess is found below the ventral process of the prootic, just lateral to the pituitary fossa (best preserved in TMM 43646-1 and TMM 47006-1) (Figs. 37.3, 55.7). Part of the prootic component of the rostral tympanic recess is found on the parabasisphenoid, but most of it is tucked behind the ventral process of the prootic (Figs. 37.3, 55.7). Coelophysoids have a single rostral tympanic recess on each side of the braincase located between the prootic and parabasisphenoid (Raath, 1977; Colbert, 1989; Rowe, 1989; Witmer, 1997). However, this recess divides such that some coelurosaurs have a prominent prootic component of the rostral tympanic recess, and other tetanurans instead exhibit a prominent subotic component of the rostral tympanic recess (Witmer, 1997). Dilophosaurus wetherilli reflects a condition where both of these components are present and are themselves subdivided. The lateral side of the braincase of Dilophosaurus wetherilli is far more pneumatic than Sinosaurus triassicus (Xing, 2012; Xing et al., 2014).

Prootic.-The sutures between the prootic and other braincase elements are difficult to determine in UCMP 37302 and UCMP 77270, but the right prootic is disarticulated in TMM 43646-1 and both elements are complete in TMM 47006-1 (Figs. 4, 37, 44.1-44.6, 56, 58.25-58.36). The prootic has dorsal and ventral processes; the dorsal process is taller than the ventral process. The trigeminal foramen $(\mathrm{CN} \mathrm{V})$ is partially enclosed between the anterior margins of the dorsal and ventral processes. The foramen is closed anteriorly by the articulation of the laterosphenoid. The ventral process of the prootic forms a broad anteroventrally oriented pendant with small anterior and posterior processes. The anterior portions of the ventral processes of the prootics meet at the midline above the dorsum sella. A pair of relatively large foramina for the right and left abducens nerves (CN VI) penetrate the dorsum sella at the top of the pituitary fossa (Fig. 57.3). The channel through which the facial nerve (CN VII) passes is present behind the trigeminal foramen just anterior to the crista prootica (Fig. 57.5). The lateral outline of the foramen for CN VII is oblong rather than circular. The anterior margin of the foramen ovale is formed by the prootic just posterior to a strut of bone that separates it from the prootic component of the rostral tympanic recess. A foramen for passage of the vesibulocochlear nerve (VIII) lies internal to the foramen ovale (visible on the left side of the articulated braincase of UCMP 77270 and well preserved on both sides of TMM 47006-1). The bony wall through which this nerve communicates forms the medial wall of the inner ear. This recess lies on the ventral process of the prootic and is divided into dorsal and ventral halves by a sharp ridge of bone (Figs. 37.3, 55.7). The dorsomedial edge of the prootic articulates with the supraoccipital and together they enclose much of the bony labyrinth.

Otooccipital.-No distinction can be made between the exoccipitals and opisthotics where they form the paroccipital process in the holotype (Fig. 4). The paroccipital processes of UCMP 37302 project posterolaterally where they are anteroposteriorly broad, dorsoventrally flattened, and broken distally. The paroccipital process of the otooccipital articulates with the medial surface of the paroccipital process of the squamosal (best preserved in UCMP 77270; Fig. 37). The descending process of the otooccipital forms the crista interfenestralis (Figs. 37.3, 55.1, $55.8,56,58.37-58.48)$. It demarcates the posterior margin of the foramen ovale and, together with the crista tuberalis of the otooccipital, forms the fenestra pseudorotunda. CT imagery of TMM 47006-1 shows that Dilophosaurus wetherilli is similar to averostrans in that the vagus nerve $(\mathrm{X})$ has a separate canal that penetrates the otooccipital below two foramina for the hypoglossal nerve (XII; Fig. 57.3). The braincase of UCMP 77270 also preserves this feature (Fig. 37.6). This is similar to the vagus foramen found in Notatesseraeraptor frickensis Zahner and Brinkmann, 2019 and Cryolophosaurus ellioti, but is in 

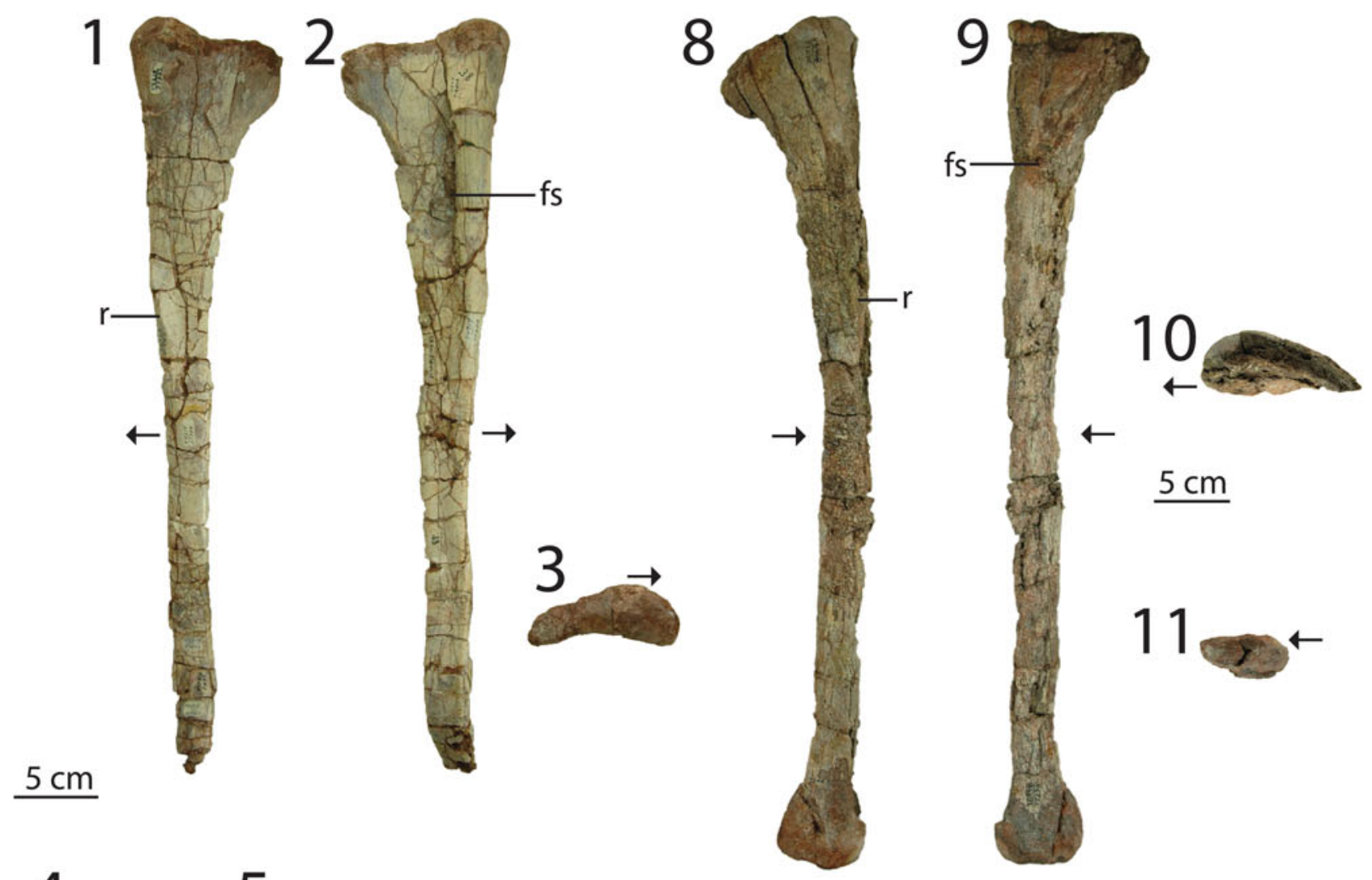

$\underline{\mathrm{cm}}$

4
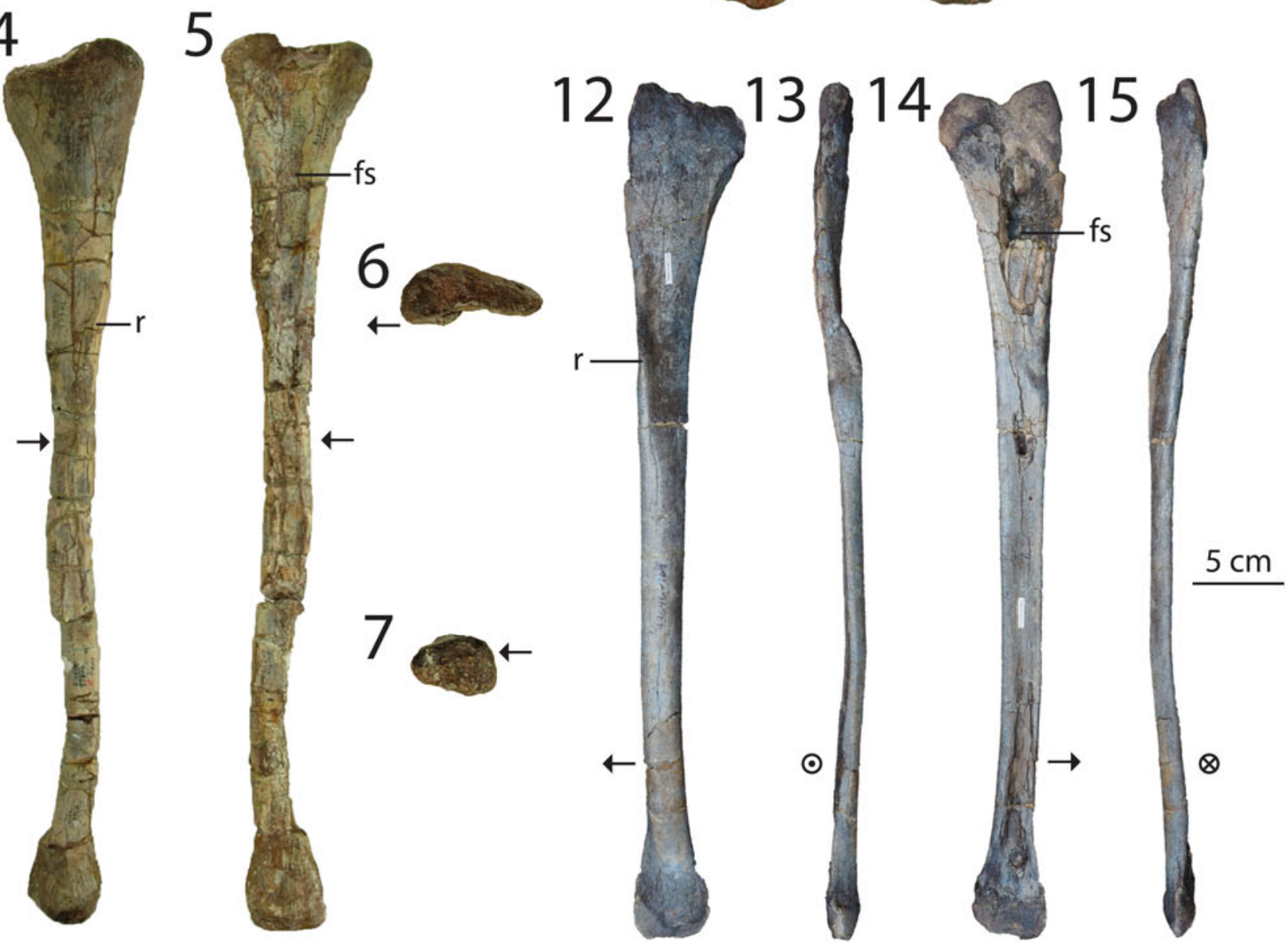

Figure 22. Dilophosaurus wetherilli holotype specimen (UCMP 37302): (1-3) left fibula and (4-7) right fibula; Dilophosaurus wetherilli referred specimen (UCMP 77270): (8-11) right fibula; Dilophosaurus wetherilli referred specimen (TMM 43646-1): (12-15) left fibula. $(\mathbf{1}, \mathbf{4}, \mathbf{8}, \mathbf{1 2})$ Lateral, (2, 5, 9, 14) medial, $(\mathbf{1 3})$ anterior, $(\mathbf{1 5})$ posterior, $(3, \mathbf{6}, \mathbf{1 0})$ proximal, and $(\mathbf{7}, \mathbf{1 1})$ distal view. Arrows point in anterior direction. $f \mathrm{~s}=$ fossa; $r=$ ridge. 
contrast to the plesiomorphic condition in which the glossopha-

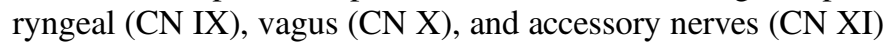
all pass through the metotic fissure (Gower and Weber, 1998; Sampson and Witmer, 2007; Knoll et al., 2012; Lautenschlager and Butler, 2016; Sobral et al., 2016; Lessner and Stocker, 2017; Chapelle and Choiniere, 2018; Paulina-Carabajal et al., 2019; Piechowski et al., 2019). The glossopharyngeal nerve (IX) penetrates the braincase near the top of the fenestra pseudorotunda, slightly anterior to the position in some tetanurans (PaulinaCarabajal and Currie, 2017). It is not clear whether cranial nerve XI or the jugular vein traversed the fenestra pseudorotunda. However, its large size and shape are more similar to the undivided metotic fissure of non-averostran theropods than to the small fenestra pseudorotunda of ceratosaurs and tetanurans. When viewed as one structure, the foramen ovale and fenestra pseudorotunda form a tall triangle with its base tucked under the crista prootica. This shape resembles more closely that of coelophysoids (Raath, 1977; Tykoski, 1998) than Sinosaurus triassicus (Xing et al., 2014), ceratosaurs (Sanders and Smith, 2005; Sampson and Witmer, 2007), and tetanurans (Madsen, 1976; Hopson, 1979; Bever et al., 2013), in which the fenestra pseudorotunda is a small, round hole. A bilateral pair of hypoglossal foramina perforate the otooccipital posterior to the crista tuberalis in a pocket formed by the paroccipital process and the exoccipital component of the occipital condyle. These foramina share that pocket with the external foramen for cranial nerve X (Fig. 55.8).

Supraoccipital.-This bone is thick and tall, and in posterodorsal view forms a sagittal tuberosity on the top of the braincase (Figs. 4, 37, 44.7-44.12, 56, 58.19-58.24). A midline ridge extends up the posterior surface of the bone. The supraoccipital flares out laterally below a sagittal eminence where it articulates with the prootics, otooccipitals, and parietals. The dorsal cerebral vein perforates the supraoccipital through a symmetrical pair of foramina just behind the deep auricular recess. In UCMP 77270, the supraoccipital is coossified tightly to the back of the parietals and the paroccipital processes of the otooccipitals. The top of the supraoccipital is subelliptical and tapers slightly anteriorly. The supraoccipital is tall, but does not extend dorsally farther than the parietals. In posterior view, the lateral walls of the supraoccipital and the posterior surface of the parietals form a pair of fossae. The deepest recess in each pocket houses the exit for the middle cerebral vein (Figs. 44.11, 56.6, 58.22). The supraoccipital in Dilophosaurus contributes to the dorsal-most margin of the foramen magnum, unlike the condition in Cryolophosaurus ellioti (Smith et al., 2007) and Allosaurus fragilis (Madsen, 1976).

Laterosphenoid.- The laterosphenoids are broken but articulated in UCMP 77270, and the left is the most complete (Fig. 37.1). The articulated braincase of TMM 47006-1 shows that the posterior edge of the prootic process encloses the front of the trigeminal foramen by articulating to the prootic in two places; it touches the front of the ventral process of the prootic to form the crista antotica, and articulates with the dorsal prootic process in front of the paroccipital process (Fig. 56). The postorbital (=capitate) process of the laterosphenoid expands into lateral and medial articular surfaces. Laterally, the laterosphenoid fits into an excised pit on the posteromedial corner of the postorbital, in the anterodorsal corner of the infratemporal fenestra. Medial to this articulation, the laterosphenoid touches the ventral surface of the frontal and almost meets the other laterosphenoid on the midline. The lateral surface of the prootic process of the laterosphenoid has a subhorizontal ridge, or the crista antotica (see TMM 43646-1, Fig. 44.23-44.40 and TMM 47006-1, Fig. 58.1-58.12).

Epipterygoid and Orbitosphenoid.-These elements are not preserved in any specimen.

Osseous labyrinth.-Cross-sections of the semicircular canals are visible in some of the broken braincases, and both inner ears are preserved perfectly in TMM 47006-1 (Fig. 57). The semicircular canals are narrow tubes that pass through the supraoccipital, otooccipital, and prootic. The anterior semicircular canal is the largest of the three; it circumscribes a tall arc that forms a right angle with the posterior semicircular canal where the two join to form the common crus (Fig. 57.7, 57.12). The posterior semicircular canal is slightly concave dorsally. The lateral semicircular canal is short; it fails to circumscribe much of an arc and is nearly straight in dorsal view. In dorsal view, the anterior semicircular canal and posterior semicircular canal meet at nearly a right angle, as in Murusraptor barrosaensis (Paulina-Carabajal and Currie, 2017) and Ceratosaurus nasicornis (Madsen and Welles, 2000). This angle is much smaller in Allosaurus fragilis (Rogers, 1999). When the inner ear is viewed with the lateral semicircular canal positioned horizontally, the common crus is inclined posterodorsally. The vestibule is subtriangular; it opens laterally through the fenestra ovale and its medial surface is formed by a thin wall of the prootic through which cranial nerve VIII passes (Figs. 57.5, 58.26, 58.32). The basilar papilla of the vestibule is relatively long. Elongation of the basilar papilla is associated with a wider range of frequency discrimination and has been observed in theropods, including therizinosaurs and tyrannosaurids (Walsh et al., 2009; Lautenschlager et al., 2012; Paulina-Carabajal and Currie, 2017). A more extreme anterior elongation has been observed in Archaeopteryx Meyer, 1861 and other birds (Alonso et al., 2004).

\section{Mandible}

Dentary.-Only the middle third of the holotype left dentary is preserved, and it contains five alveoli (Fig. 5.5-5.8). Two teeth remain in place and have serrated carinae along only their distal edges. Each dentary of UCMP 37303 has 17 alveoli (Fig. 27.1-27.5), and those of UCMP 77270 preserve 16 (Fig. 38); the anterior teeth are too broken to see serrations, but a preserved posterior tooth is serrated on its mesial and distal edges, and the incomplete right dentary of TMM 43646-1 preserves 13 or 14 alveoli (Fig. 45.1-45.6). An alveolar row of foramina follows the labial margin of the tooth row and these are all connected via a shallow curving groove extending behind dentary tooth five. A pair of small foramina is found on the anteroventral margin of the dentary. The alveoli of the paratype, UCMP 77270, and TMM 43646-1 are separated by transverse walls of bone. Lingual interdental plates are present, but they are not coossified to one another. A nutrient groove incises the dentary lingual to the interdental plates, and from it occasional foramina penetrate between individual plates.

The anterior tip of the dentary is swollen both laterally and dorsally, forming what Welles (1984) called a 'chin' (Figs. 27.1, 

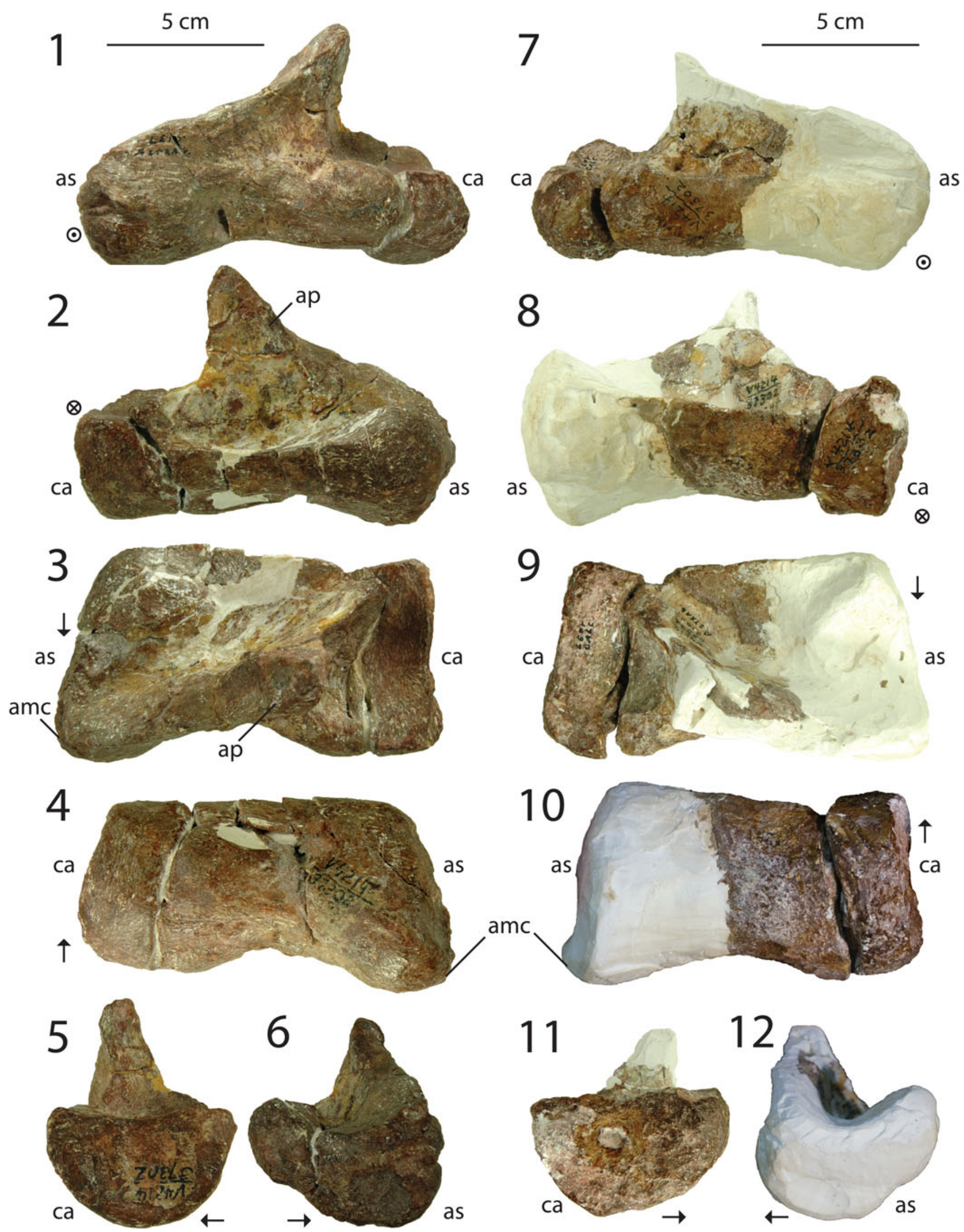

Figure 23. Dilophosaurus wetherilli holotype specimen (UCMP 37302): (1-6) left astragalocalcaneum and (7-12) right astragalocalcaneum in (1, 7) anterior, (2, 8) posterior, $(\mathbf{3}, \mathbf{9})$ proximal, $(\mathbf{4}, \mathbf{1 0})$ distal, $(\mathbf{5 , 1 1})$ lateral, and $(\mathbf{6 , 1 2})$ medial view. Arrows point in anterior direction. amc $=$ anteromedial corner; ap $=$ ascending process; as $=$ astragalus; $\mathrm{ca}=$ calcaneum. 
$27.3,38.1,38.8)$. This is in contrast to the tip of the dentary of Sinosaurus triassicus (Xing, 2012) and coelophysoids (Colbert, 1989; Rowe, 1989), which is not greatly expanded. The dentary is pinched behind the 'chin' at the sixth dentary tooth. The anterior end of the dentary is flattened medially to form a ligamentous symphysis that extends the length of the first three teeth. The dentary flares dorsally above the eighth tooth and then continues posteriorly as a more-or-less straight, horizontal edge. The posterior end of the dentary is bifurcated by the anterior-most margin of the mandibular fenestra (best preserved in TMM 43646-1).

The Meckelian groove begins at an elongate foramen under tooth four and widens posteriorly to the back of the dentary. The groove widens most dramatically beneath dentary tooth 11 . Between dentary teeth 12 and 13 on the right dentary of UCMP 37303, two large circular foramina open on the medial surface into the Meckelian groove; the dorsal foramen is deeper and slightly anterior to the ventral foramen. The foramina do not perforate the lateral surface of the dentary. A short horizontal ridge separates the two foramina within the Meckelian groove. This feature represents bilateral asymmetry in UCMP 37303 (Fig. 27.2, 27.4); other preserved dentaries only exhibit one foramen (Figs. 38.3, 45.5).

Splenial and coronoid.-When in articulation, the right splenial of UCMP 37303 covers most of the Meckelian groove, including the pair of foramina in the Meckelian groove of the dentary (Fig. 27.4, 27.6, 27.7). The splenial has its own foramen near the position of dentary tooth 12 . This foramen is also present in UCMP 77270 where it pierces the anterior corner of the splenial medial to dentary tooth 11 when the two elements are articulated (Fig. 38.3, 38.9). The splenial forms an isosceles triangle whose obtuse corner points dorsally to the posterior terminus of the mandibular tooth row. It is thickened ventrally where it articulates with the bottom of the dentary, and it thins dorsally. The entire bone is convex laterally. The coronoid is not preserved in any specimen.

Surangular.-The posterolateral end of the surangular has a thick horizontal ridge of bone that extends from a point above the mandibular fenestra posteriorly to form a pyramidal dorsal process where it meets the articular (Figs. 5.1-5.4, 38, 45.5, 45.6). The horizontal ridge forms a shelf that curves downwards to enclose a ventral groove. The pyramidal dorsal process structure found on the posterior end of the shelf has a flat surface that points dorsomedially, towards the quadrate-articular joint in an orientation similar to that of Dracovenator regenti (Yates, 2005). In UCMP 77270, a prominent trapezoidal dorsal process lies in front of the articular anterior to the pyramidal process next to the jaw joint on the left surangular, but this trapezoidal process is not present on the right surangular (Fig. 38.2, 38.5, $38.8,38.11)$. Owing to the abnormal preservation of cartilaginous structures between the sacral vertebrae of that specimen, this structure may be a calcified remnant of the jaw adductor musculature. The trapezoidal process bends slightly medially and encloses a smooth groove against the posterodorsal edge of the surangular. The surangular contributes to the retroarticular process of the mandible, which is a compound element also formed by the articular. The retroarticular process of the surangular curves down from the pyramidal process where it widens and coosifies with the back of the articular (Fig. 5.1, 5.2). The surangular is thickened dorsally and wraps up and over to form the dorsomedial surface of the mandible behind the tooth row. The contribution of the surangular to the craniomandibular articulation can be seen in dorsal view as a transverse depression that articulates with the medial condyle of the quadrate. The contribution of the surangular to the mandibular fenestra is small and not well preserved in any specimen.

Angular.- The angular tapers posteriorly and curves ventrally where it contributes to the retroarticular process (Figs. 5.1-5.4, 38). The ventral edge of the angular is thickened and has a longitudinal groove for the reception of the prearticular, as the latter extends under from the medial side. The anterodorsal margin of the angular contributes to the bottom of the mandibular fenestra. The mandibular fenestra is elongate and relatively small, as is the case in Syntarsus kayentakatae (Rowe, 1989; Tykoski, 1998) and Zupaysaurus rougieri (Ezcurra, 2006). The angular extends backwards far enough to exclude the surangular from the ventral margin of the mandible.

Articular and prearticular.-The articular is difficult to distinguish from the retroarticular process of the surangular with which it is coossified in UCMP 37302 (Fig. 5.3, 5.4). A transverse elliptical articular facet on the dorsal surface articulates with the medial condyle of the quadrate. Both mandibles of UCMP 77270 are crushed in this region, but it seems that the articular and prearticular are coossified medially (Fig. 38). The prearticular is concave-up in medial view (Fig. 38). The posteroventral edge of the prearticular is fairly straight in front of the retroarticular process. It thins anteriorly until it simultaneously expands dorsoventrally and curves upward. In Dilophosaurus, the quadrate-articular joint lies behind the dorsal head of the quadrate, whereas the joint lies directly beneath the dorsal head of the quadrate in coelophysoids and Zupaysaurus rougieri (Raath, 1977; Colbert, 1989; Rowe, 1989; Ezcurra, 2006).

\section{Vertebral column}

Atlas-axis.-The proatlas is an elongate flat bone that is elliptical in outline and arches over the anterior atlas-axis complex (Fig. 6.7-6.9). This bone has a short dorsal midline bump. The proatlas abuts the occiput just above the foramen magnum and would loosely articulate with the neurapophyses of the atlas.

The atlantal intercentrum is broad anteriorly (Figs. 6.11$6.16,39)$. The anterodorsal surface is strongly depressed and terminates anteriorly at a lipped edge. The ventral surface is excavated along the midline to form a lateral tuber on each ventrolateral corner. The dorsal articulation with the atlantal pleurocentrum is gently concave. In lateral view, the atlantal intercentrum is subrectangular. The lateral surface of the bone has a curved vertical groove and a small dorsal notch. A semicircular fossa is formed between the atlantal intercentrum and pleurocentrum that receives the occipital condyle (Fig. 6.13). The floor of the endocranial cavity is continuous with a concave surface on the atlantal pluerocentrum (odontoid process), where the spinal cord passes to the neural canal. The atlantal plurocentrum is shorter than the atlantal intercentrum and is subcubic in shape (Fig. 6.11-6.16). The atlantal pleurocentrum comes to a point along the dorsal midline and from this point the anterior surface slopes downward away from this feature. Circular fossae occupy most of the lateral surfaces of the atlantal pleurocentrum. The atlantal pleurocentrum and axial intercentrum are coossified 


\section{1}
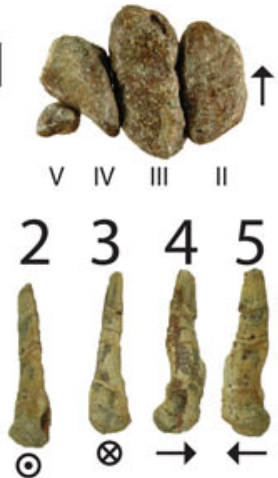

$14 \quad 15,16$
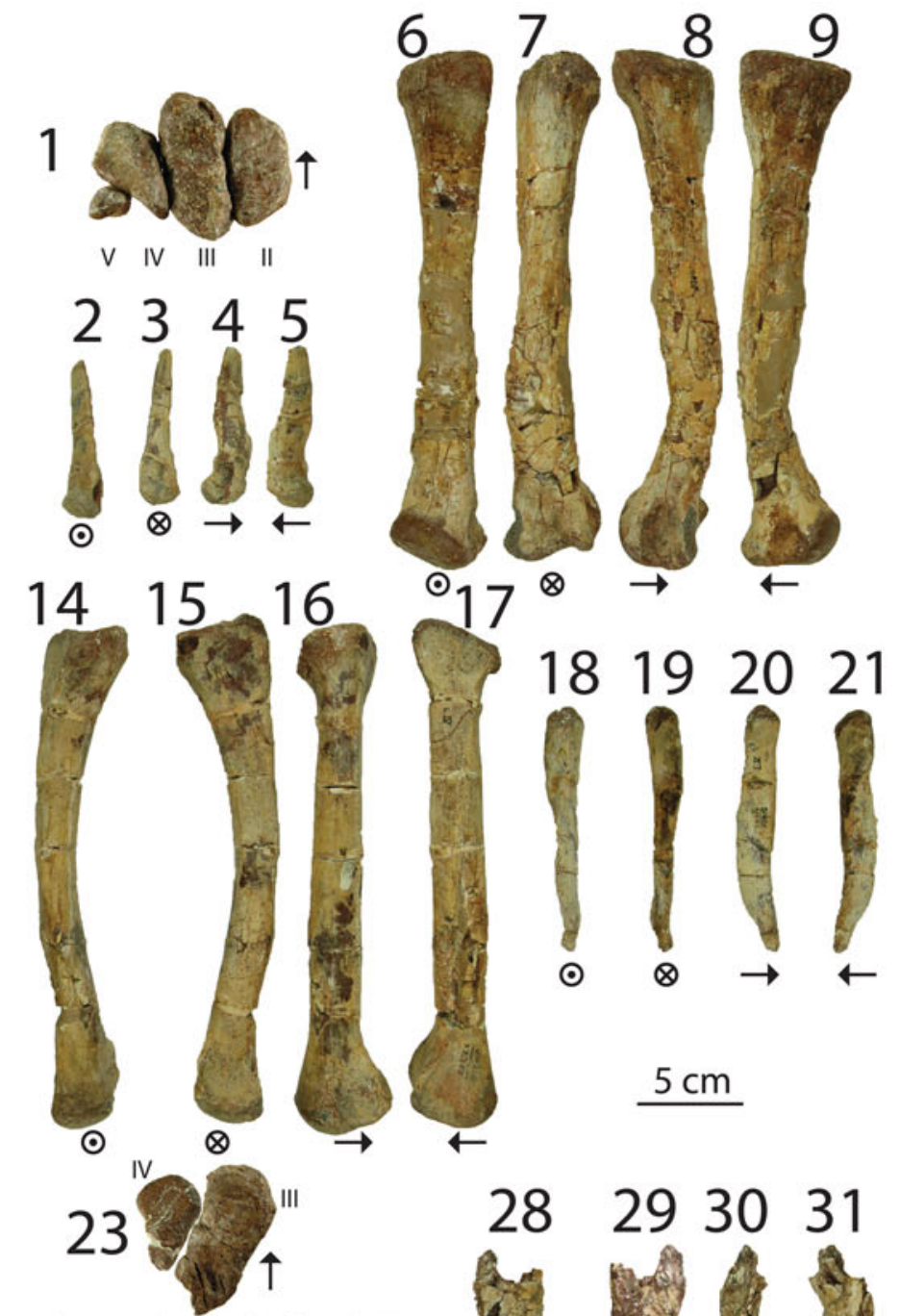

24252627
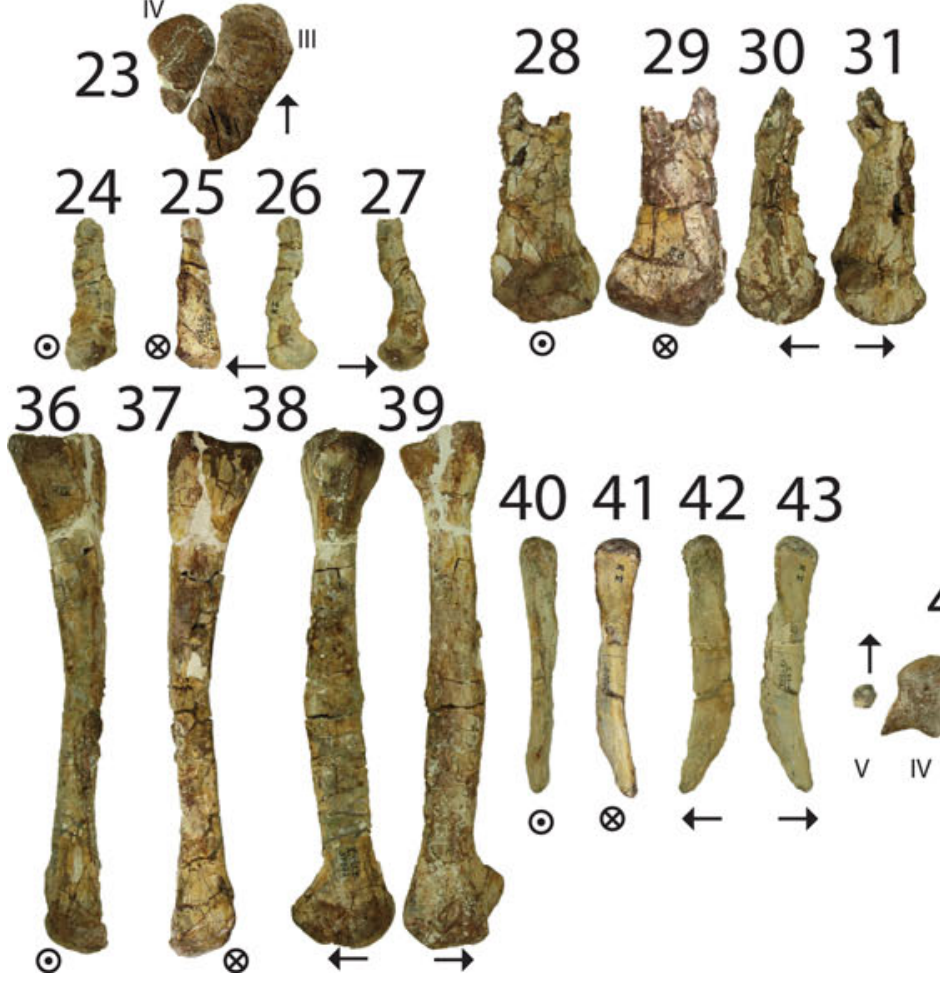

$\odot$
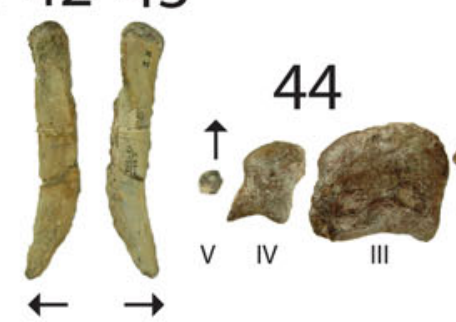

○

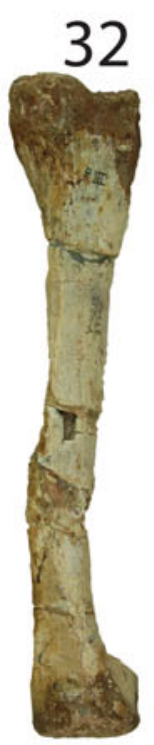

$\otimes$
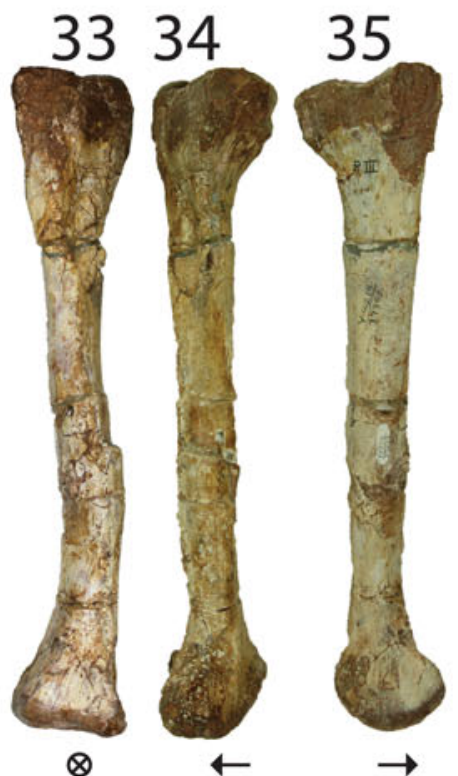

Figure 24. Dilophosaurus wetherilli holotype specimen (UCMP 37302): (2-5, 22) left metatarsal I, (1, 6-9, 22) left metatarsal II, (1, 10-13, 22) left metatarsal III, $(\mathbf{1}, \mathbf{1 4}-\mathbf{1 7}, \mathbf{2 2})$ left metatarsal IV, $(\mathbf{1}, \mathbf{1 8}-\mathbf{2 2})$ left metatarsal V, $(\mathbf{2 4 - 2 7}, \mathbf{4 4})$ right metatarsal I, $(\mathbf{2 8}-\mathbf{3 1}, \mathbf{4 4})$ right metatarsal II, $(\mathbf{2 3}, \mathbf{3 2}-\mathbf{3 5}, \mathbf{4 4})$ right metatarsal III, $(\mathbf{2 3}$, 36-39, 44) right metatarsal IV, (40-44) right metatarsal V, $(\mathbf{4 5}, \mathbf{4 6})$ left distal tarsal IV, $(\mathbf{4 7}, \mathbf{4 8})$ right distal tarsal IV, and $(\mathbf{4 9}, \mathbf{5 0})$ right distal tarsal III in $(\mathbf{2 ,}, \mathbf{6}, \mathbf{1 0}, \mathbf{1 4}$, $18,24,28,32,36,40)$ dorsal, $(3,7,11,15,19,25,29,33,37,41)$ ventral, $(4,8,12,16,20,26,30,34,38,42)$ lateral, $(5,9,13,17,21,27,31,35,39,43)$ medial, $(1,23$, $\mathbf{4 6}, \mathbf{4 8}, \mathbf{5 0})$ proximal view, and $(\mathbf{2 2}, \mathbf{4 4}, \mathbf{4 5}, \mathbf{4 7}, \mathbf{4 9})$ distal view. Arrows point in dorsal direction. 
in UCMP 77270 and form a large odontoid process on the end of the axial pleurocentrum (Fig. 39).

The neural arch of the atlas comprises two separate neurapophyses that fuse on the dorsal midline (Figs. 6.1-6.6, 46). Each arch is made up of a curved flange of bone that is concave medially where it surrounds the spinal cord. A short dorsomedial stalk projects anteriorly and expands into a reniform shape that articulates with the sides of the occipital condyle. There is no articular facet for the proatlas. The atlantal postzygapophysis is elliptical, flat, and faces ventrally. A strong epipophysis projects dorsolaterally from the back of the postzygapophysis.

The axial intercentrum is a triangular wedge located between the atlantal intercentrum and axial pleurocentrum (Fig. 6.11, 6.12, 6.16). In ventral view, the anterior and posterior margins of the axial intercentrum curve backwards, away from the midline. The axial pleurocentrum makes up the main body of the axis (Figs. 6.11-6.16, 39, 46, 59.2). Anteriorly, lateral depressions receive the curved axial intercentrum and the anterodorsal margin is slightly excavated to receive the atlantal pleurocentrum (Fig. 6.11). The ventral surface of the axial centrum is strongly keeled. The ventral keel is most prominent anteriorly, but it becomes confluent with the body of the centrum approximately halfway down its length. Small anteroventral tubera are found on the rim of the centrum beneath where the axial intercentrum articulates. Posteriorly, the face of the centrum is circular and strongly concave (i.e., opisthocoelous). The articulation of the neural arch with the centrum is convex dorsally. The pedicles of the neural arch slope down the back of the centrum. The neural canal is circular anteriorly and forms a tall triangle in posterior view. The prezygapophyses are short and face dorsolaterally. A low lamina of bone connects the prezygapophysis and postzygapophyses laterally. Pointed epipophyses project backwards well beyond the postzygapophyses.

The neural spine is very tall and broad, unlike the more diminuitive axial spine of averostrans (Nesbitt, 2011). Anteriorly it rises from the prezygapophyses and curves forward to a point (Figs. 6.11, 39). The spine continues backwards as it rises further, and, at its tallest point, it splits into the divergent spinopostzygapophyseal laminae (Figs. 6.14, 59.2). Each lamina descends to the epipophysis. The infrapostzygapophyseal lamina is well developed posteriorly and demarcates the floor of the spinopostzygapophyseal fossa.

Post-axial cervical vertebrae.-In Dilophosaurus wetherilli, the cervical vertebrae are relatively short anteroposteriorly compared to the those of Tawa hallae (Nesbitt et al., 2009b), Eodromaeus murphi (Martínez et al., 2011), coelophysoids (Raath, 1977; Colbert, 1989; Rowe, 1989; You et al., 2014), Liliensternus liliensterni (von Huene, 1934), and Zupaysaurus rougieri (Ezcurra and Novas, 2007), but are similar in anteroposterior length to those of Cryolophosaurus ellioti (Smith et al., 2007). There are four major lateral laminae and fossae on the neural arch of vertebra 3 (Figs. 39, 59.3). The centrum is opisthocoelous and has a ventral keel on its anterior end. The neural spine is incomplete in all specimens.

Cervical vertebra 4 preserves both the anterior and posterior pleurocoels on the ventral half of the lateral sides of the centrum (Figs. 7, 39, 46, 59). The posterior pleurocoel is dorsoventrally wide, but not very deep, whereas the anterior pleurocoel excises deeply into the front of the centrum. The fourth vertebra has a very short midline ventral keel (Fig. 9). Additionally, anterolateral tubera project below the parapophysis on either side of the keel, and each has a short ridge behind it. The centrum is opisthocoelous. The prezygapophysis is prominent and mediolaterally wide, forming a broad paddle that faces dorsomedially. The postzygapophysis is slightly higher than the prezygapophysis and is topped by a prominent, pointed epipophysis (Figs. 7-9). On the lateral side of the neural arch, the transverse process is low and extends down to nearly reach the parapophysis.

Prezygadiapophyseal, postzygadiapophyseal, and anterior centrodiapophyseal laminae are present on the neural arch. They connect to the downwards-sloping diapophysis (Fig. 7). On the fourth vertebra, the posterior centrodiapophyseal lamina bifurcates anteriorly to form two lower laminae between which lies an additional fossa (Fig. 62.1-62.4). By the fifth vertebra, each of the lower laminae are themselves divided posteriorly into two more sublaminae (Fig. 62.5). This splits the postzygadiapophyseal fossa into two smaller fossae. This condition is similar on the sixth vertebra (Fig. 62.6), but on the seventh and eighth vertebra the two sublaminae are undivided and the condition resembles that of the fourth vertebra (Fig. 62.7, 62.8). On the ninth vertebra, the two lower laminae unite to reform the posterior centrodiapophyseal lamina and postzygadiapophyseal fossa (Fig. 62.9). This serial change of the posterior centrodiapophyseal lamina is not well developed in TMM 47006-1 (although it is split on cervical vertebra 4; Fig. 59.3), suggesting that it indicates an ontogenetic change in relation to an increase in body size or perhaps some other source of intraspecific variation.

The neural spine of the fourth vertebra is subrectangular in lateral view, but its anterodorsal and posterodorsal corners project out from the spine (=anterior shoulder and posterior shoulder; Welles, 1984; Figs. 7, 63.1). The spine is topped by a squared process that thickens dorsally (=the 'cap' of Welles, 1984; Figs. 7, 9, 46.6, 46.7, 61.1, 63). This structural arrangement is also present in the sauropodomorph Sarahsaurus aurifontanalis (Rowe et al., 2011). Spinoprezygapophyseal and spinopostzygapophyseal fossae are present and extend well beneath the neural spine (Fig. 8). They also extend up the anterior and posterior edges of the neural spine to the 'shoulders' and form the spinoprezygapophyseal and spinopostzygapophyseal laminae, respectively. The prezygapophyseal centrodiapophyseal fossa, centrodiapophyseal fossa, and postzygapophyseal centrodiapophyseal fossa are deeply excavated into the side of the neural arch, but the spinodiapophyseal fossa is only a shallow concavity on the anterolateral surface of the transverse process (Figs. 7, 8). The centroprezygapophyseal fossa is housed between the prezygapophysis, the infraprezygapophyseal lamina, and unnamed laminae bordering the neural canal. The prezygadiapophyseal, postzygadiapophyseal, and anterior centrodiapophyseal laminae are present, as are the prezygapophyseal centrodiapophyseal, centrodiapophyseal, postzygapophyseal centrodiapophyseal, and spinodiapophyseal fossae.

The anterior pleurocoels do not connect with one another medially in a centrocoel (Welles, 1984; Figs. 7, 39, 59, 64). The middle of the centrum is hollow, it is just not confluent with the fossa that incise the lateral wall of the centrum as do the pneumatic excavations of averostrans like Allosaurus fragilis 


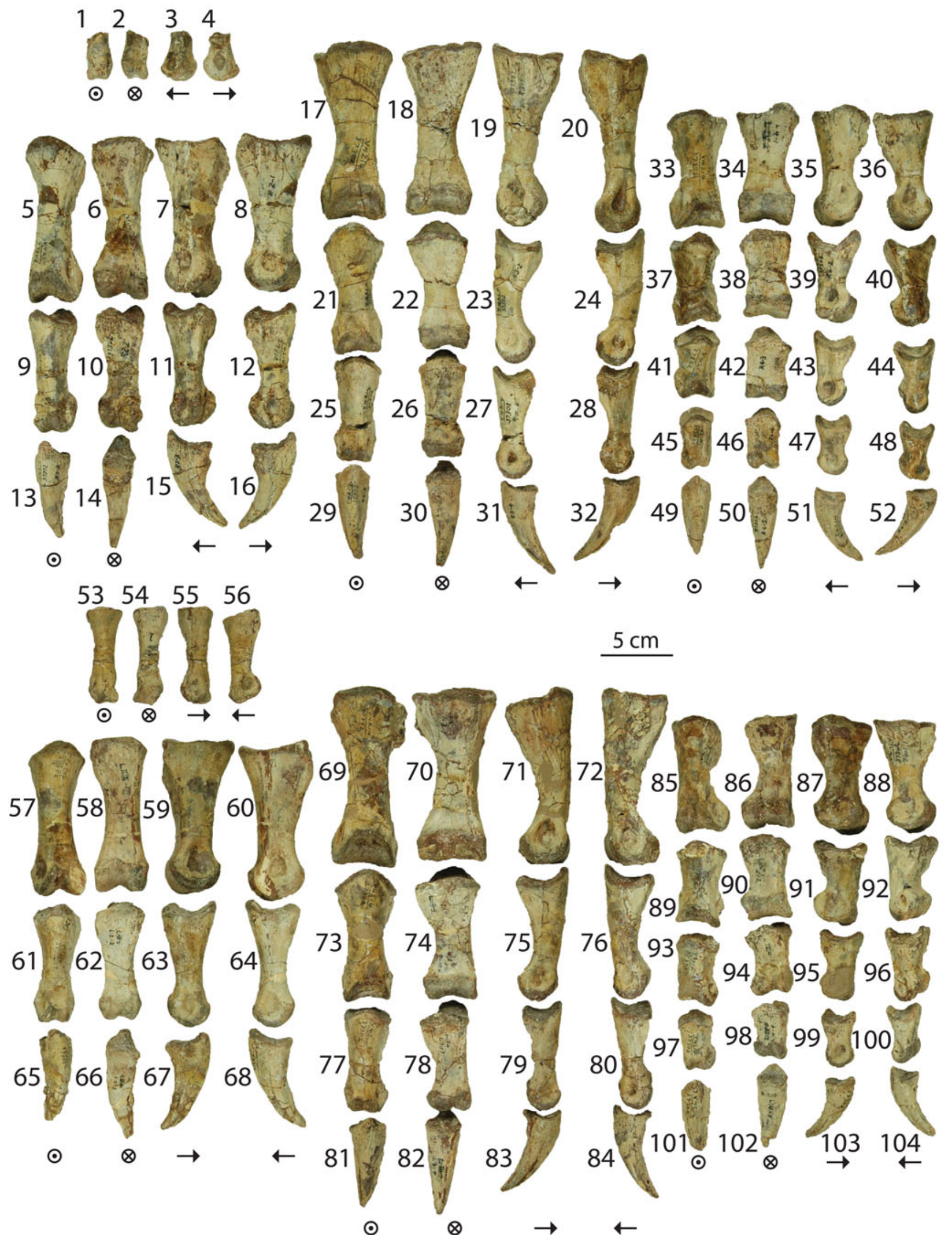


Figure 25. Dilophosaurus wetherilli holotype specimen (UCMP 37302): (1-4) left pedal phalanx I-1, (5-8) left pedal phalanx II-1, (9-12) left pedal phalanx II-2, (13-16) left pedal phalanx II-3, (17-20) left pedal phalanx III-1, (21-24) left pedal phalanx III-2, (25-28) left pedal phalanx III-3, (29-32) left pedal phalanx III-4, (33-36) left pedal phalanx IV-1, (37-40) left pedal phalanx IV-2, (41-44) left pedal phalanx IV-3, (45-48) left pedal phalanx IV-4, (49-52) left pedal phalanx IV-5, (53-56) right pedal phalanx I-1, (57-60) right pedal phalanx II-1, (61-64) right pedal phalanx II-2, (65-68) right pedal phalanx II-3, (69-72) right pedal phalanx III-1, (73-76) right pedal phalanx III-2, (77-80) right pedal phalanx III-3, (81-84) right pedal phalanx III-4, (85-88) right pedal phalanx IV-1, (89-92) right pedal phalanx IV-2, (93-96) right pedal phalanx IV-3, (97-100) right pedal phalanx IV-4, (101-104) right pedal phalanx IV-5 in $(\mathbf{1}, \mathbf{5}, \mathbf{9}, \mathbf{1 3}, \mathbf{1 7}, \mathbf{2 1}, \mathbf{2 5}, \mathbf{2 9}, \mathbf{3 3}, \mathbf{3 7}, \mathbf{4 1}, \mathbf{4 5}, \mathbf{4 9}, \mathbf{5 3}$, $57,61,65,69,73,77,81,85,89,93,97,101)$ dorsal, $(2,6,10,14,18,22,26,30,34,38,42,46,50,54,58,62,66,70,74,78,82,86,90,94,98,102)$ ventral, $(3,7,11$,

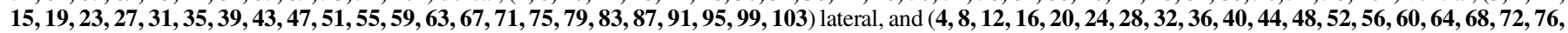
$\mathbf{8 0}, \mathbf{8 4}, \mathbf{8 8}, 92,96,100,104)$ medial view. Arrows point in dorsal direction.

(Madsen, 1976), Piatnitzkysaurus floresi (Bonaparte, 1986), Ceratosaurus nasicornis (Madsen and Welles, 1976), and Majungasaurus crenatissimus (Sampson and Witmer, 2007; Fig. 64). The fifth vertebra has a circular midline fossa on the front of the ventral surface instead of a keel (Fig. 64.2). Two ridges brace the anteroventral tubera on either side of this fossa and form secondary ventrolateral fossae between ridges and the parapophysis. The same structures are found on the bottom of vertebrae 6 and 7, but the ridges become closer to one another towards the midline (Fig. 64). By the eighth vertebra, the anteroventral ridges combine to form a low anterior keel. This keel becomes more prominent and extends posteriorly on vertebra 9 and by the tenth vertebra it almost reaches the back of the centrum (Fig. 64.3). The fifth vertebra has a neural spine topped with a 'cap' that has two posterolateral grooves behind it (Figs. 7, 9, 63).

The sixth vertebra is opisthocoelous and the anterior surface of the centrum is flat (Figs. 7, 8, 39, 59.9-59.14). The parapophysis is situated on the ventrolateral margin of the front face of the centrum in front of the deeply excavated anterior pleurocoel. The ventral surface of the centrum is concave behind its front rim, but then descends ventrally. An elliptical posterior pleurocoel is excised into the back of the lateral centrum walls (Fig. 64). In anterior view, an unnamed oblique lamina extends from the medial base of the pedicle between the neural canal and the centroprezygapophyseal lamina, and projects dorsomedially to touch the base of the infraprezygapophyseal lamina (Fig. 59.11). Parasagittal triangular centroprezygapophyseal fossae (=prechonos of Welles, 1984) are outlined by these unnamed laminae, the centroprezygapophyseal laminae, and the medial wall of the prezygapophyses. The same lamina and fossa are found on the lateral sides of the neural arch as those on cervical four, including the divided posterior centrodiapophyseal lamina (Figs. 39, 62). The shape and relative size of the prezygapophysis, postzygapophysis, epipophysis, neural spine, spinoprezygapophyseal fossa, and spinopostzygapophyseal fossa are similar to those on the fourth vertebra, but they are slightly larger on cervical six.

The epipophysis of the seventh vertebra is pointed and projects far behind the postzygapophysis (Figs. 8, 39, 60.5). The major differences between this vertebra and those that precede it are located on the neural spine. The spinoprezygapophyseal and spinopostzygapophyseal fossae excise into the base of the neural spine of the sixth vertebra (Fig. 63), but on the seventh cervical vertebra they widen and cause the anterior and posterior edges of the spine to flare laterally at the 'shoulder.' Further, the dorsal 'cap' of the neural spine widens mediolaterally and bifurcates anteriorly to create two short processes (Fig. 63).
The spinoprezygapophyseal and spinopostzygapophyseal laminae expand further anterolaterally on the neural spine of the eighth cervical vertebra (Fig. 63), and the 'cap' becomes blockier and its posterior end also becomes divided medially. Two sharp, low ridges form a groove that extends from the back of the 'cap' down the posterolateral side of the neural spine.

The posterior face of the ninth centrum is convex (Figs. 8, 39). The transverse process of this vertebra is narrow, projects laterally, and is subtriangular in distal outline. The prezygapophysis and postzygapophysis are located more laterally than in more anterior vertebrae. An accessory pneumatic fossa is found within the prezygapophyseal centrodiapophyseal fossa under the transverse process near its distal end (Fig. 39). This fossa is subelliptical and is much deeper in the ninth vertebra than the tenth.

The tenth cervical vertebra does not have posterior pleurocoels (Figs. 7, 39, 64). The neural spine is strongly cruciform in dorsal view (Figs. 9, 63.3). The spinoprezygapophyseal and spinopostzygapophyseal laminae are lower anteriorly and form a shallow pocket. The 'cap' is divided anteriorly and posteriorly, forming two symmetrical triangles (Fig. 63). The posterior margin of the rear triangle extends down the lateral side of the spine towards the postzygapophysis and overhangs a lateral fossa posteroventral to the 'cap.'

The anterior pleurocoel of the eleventh vertebra is situated more dorsally than those of more anterior vertebrae (Figs. 39, 64). The parapophysis is found on the upper third of the anterior margin of the centrum and is braced from behind by a broad lamina under the anterior pleurocoel. A low tuber projects posterolaterally below the parapophysis on the anterolateral rim of the centrum. An undivided, low ventral keel is present along the entire length of the centrum (Fig. 64). The anterior face of the centrum is flat and projects farther ventrally than the posterior face, which is slightly concave.

The anterior and posterior faces of the twelfth vertebra are equal in height and are not offset dorsoventrally from one another in lateral view (Figs. 7, 8). A midline ventral keel is present and anterolateral tubers are found on the ventral surface of the centrum adjacent to the keel anteriorly (Fig. 64). The transverse process projects dorsolaterally. The neural arch comprises four laminae: the anterior and posterior centrodiapophyseal laminae, the prezygadiapophyseal lamina, and the postzygadiapophyseal lamina (Fig. 62).

The centrum of the thirteenth vertebra is much like the two before it except it has lost the anterolateral tuber on the front of the centrum and the ventral keel is only present anteriorly (Fig. 64). A hyposphene juts out posteroventrally above the neural canal beneath the postzygapophyses (Fig. 8). The 

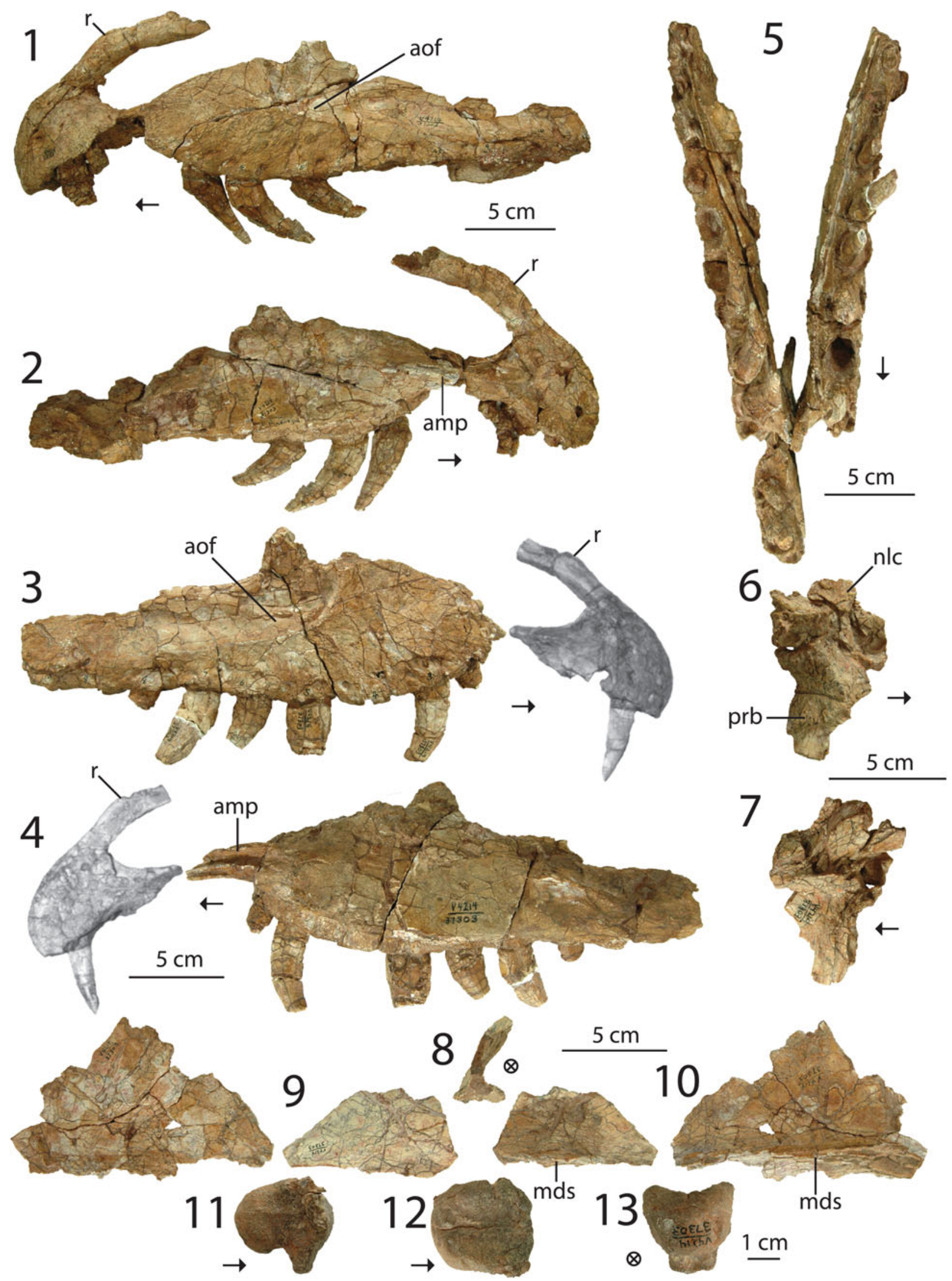

Figure 26. Dilophosaurus wetherilli paratype specimen (UCMP 37303): (1, 2, 5) articulated left maxilla and premaxilla, (3-5) right maxilla and premaxilla, (6, 7) right lacrimal, (8-10) two pieces of the nasolacrimal crests, and (11-13) basioccipital in $(\mathbf{1}, \mathbf{3 , 6}, \mathbf{9 , 1 1})$ lateral, $(\mathbf{2}, \mathbf{4 , 7}, \mathbf{1 0})$ medial, $(\mathbf{1 2})$ dorsal, $(\mathbf{5})$ ventral, and $(\mathbf{8 , 1 3})$ posterior view. Arrows point in anterior direction. $\mathrm{amp}=$ anteromedial process; aof = antorbital fossa; $\mathrm{mds}=$ midline suture; nlc = nasolacrimal crest; prb = preorbital boss; $r=$ ridge. 
zygapophysis is closer to the midline of the vertebra and is not as prominent as that of previous vertebrae.

The fourteenth vertebra is the last cervical vertebra; the parapophysis remains on the centrum in all of the specimens that preserve this vertebra (e.g., UCMP 37302; Fig. 7) with the exception of TMM 47006-1. On that specimen, the right parapophysis is on the centrum and the left parapophysis is on the neural arch (Fig. 60.7). The ventral keel on the centrum of the fourteenth vertebra is only present on the most anterior margin of the centrum (Fig. 64). The anterior pleurocoel is very shallow behind the parapophysis (Fig. 64). The hyposphene is prominent and flares ventrally in posterior view. The neural spine is dorsoventrally longer, but the 'cap' is still robust mediolaterally (Fig. 63). The lateral triangles that are formed by the 'cap' anteriorly in the neck are combined in the fourteenth vertebra into a single unit. The posterolateral fossa is not present behind this structure on the fourteenth vertebra.

Trunk vertebrae.-The anterior and posterior faces of the trunk centra are approximately equal in size and shape. The trunk centra are amphiplatyan, if not slightly amphicoelous (Figs. 10, 40.3-40.6, 47.1, 60.7-60.11). The posterior trunk centra are longer relative to the height of their anterior articular surfaces than those of Cryolophosaurus ellioti and early averostrans (Smith et al., 2007; Nesbitt and Ezcurra, 2015), but not as long as those of some coelophysoids (Raath, 1977; Colbert, 1989). Low midline ventral keels are present on the front half of the anterior two trunk vertebrae (the fifteenth and sixteenth; Fig. 64). Extensive lateral excavations are absent on the trunk centra. Longitudinal striations parallel to the long axis of the centrum are present adjacent to the rims of the centra on both ends of each vertebra except the anterior end of the fifteenth vertebra (Fig. 64).

The parapophysis lies on the anteroventral corner of the neural arch pedicle on the fifteenth vertebra (Figs. 10, 40, 60.7). The parapophysis is taller than it is wide in the anterior trunk vertebrae, but it becomes circular in the mid-trunk region where it projects laterally on short stalks. Similar 'stalks' are present in Cryolophosaurus ellioti (Smith et al., 2007) and Ceratosaurus nasicornis (Madsen and Welles, 2000). The distal end of the diapophysis is subtriangular and dorsoventrally shortened. Throughout the dorsal series, the prezygapophysis is shorter than that of the cervical vertebrae, but still hangs over the front of the centrum (Fig. 10). In dorsal view, the prezygapophysis is rounded anteriorly and its medial edge is close to the midline, bracketing the lateral wall of the hypantrum. The postzygapophysis is anteroposteriorly short. Hyposphenehypantrum articulations are variably preserved in the holotype owing to preservation. Because the parapophysis is situated closer to the diapophysis in more posterior trunk vertebrae, the transverse process becomes anteroposteriorly wider distally. In dorsal view, the transverse process is tapered laterally, flat dorsally, and swept back posterolaterally. The transverse processes points laterally rather than posterolaterally in posterior trunk vertebrae.

The neural spine is present on some of the holotype vertebrae and exhibits transitional shapes from anterior to posterior vertebrae (Figs. 10, 63). Anteriorly, the neural spine comprises the anterior and posterior 'shoulders' as well as the thickened dorsal 'cap' (Welles, 1984). The top of the spine of the fifteenth vertebra is wider than that of the posterior cervical neural spines, and the 'shoulders' are more prominent and pointed in lateral view. The dorsal 'cap' is wider than the rest of the neural spine posteriorly and tapers anteriorly. The anterior shoulder of the sixteenth neural spine is confluent with the top of the dorsal 'cap' and forms a convex dorsal margin. The 'cap' is thick posteriorly and a small posterior 'shoulder' extends behind it. This 'shoulder' is not present on the following neural spine (the seventeenth vertebra). Instead, the neural spine itself is a long blade-like structure with a pointed anterior process. The anterior process becomes smaller and the neural spine becomes higher in the posterior trunk vertebrae. By the $22^{\text {nd }}$ vertebra, the neural spine is a tall, long process with a slight dorsal convexity and pointed posterior projection. The rounded dorsal margin of the posterior trunk neural spines is shared with Herrerasaurus ischigualastensis (Novas, 1994) and Eoraptor lunensis (Sereno et al., 2012).

Paired spinoprezygapophyseal and spinopostzygapophyseal laminae (and their associated midline fossa) are present on the trunk vertebrae, but the intraprezygapophyseal and intrapostzygapophyseal laminae do not occur because of the development of the hyposphene-hypantrum articulations in the posterior cervical and trunk vertebrae. The most obvious change to the lateral vertebral laminae appears as the parapophysis is situated further up on the neural arch (Figs. 10, 60.7). In doing so, it tracks the path of the anterior centrodiapophyseal laminae, splitting that lamina into two laminae where the parapophysis occurs. Instead of the anterior centrodiapophyseal lamina, the fifteenth vertebra only has the paradiapophyseal lamina. The sixteenth through twentieth vertebrae have a ventral anterior centroparapophyseal lamina and dorsal paradiapophyseal laminae. The centroprezygapophyseal lamina merges with the parapophysis and becomes the prezygaparapophyseal lamina where the parapophysis is close to the front of the pedicle (fifteenth through seventeenth vertebrae). The parapophysis merges with the prezygadiapophyseal lamina to form the prezygaparapophyseal lamina. The posterocentrodiapophyseal lamina is present throughout the trunk vertebrae. The prezygapophyseal centrodiapophyseal fossa becomes reduced in size in posterior trunk vertebrae (and is actually the prezygapophyseal paradiapophyseal fossa in the fifteenth through twentieth vertebrae), but the postzygapophyseal centrodiapophyseal fossa extends farther laterally behind the transverse processes. A pneumatic fossa excavates the underside of the base of the transverse processes of the sixteenth through twentieth vertebrae within the centrodiapophyseal fossa, forming two accessory laminae (Fig. 62). One lamina projects posteroventrally from the back of the parapophysis and the other extends anteroventrally from where the posterior centrodiapophyseal lamina turns laterally to travel down the transverse process. Depending on the specimen and the side of the vertebra, either of these laminae is more pronounced than the other. These are preserved in the sixteenth through nineteenth vertebrae of UCMP 37302 and the nineteenth and twentieth vertebrae of UCMP 37303 and UCMP 77270 .

Sacral vertebrae.-All of the sacral centra are amphiplatyan. The striations present adjacent to the rims of the dorsal centra are found on the posterior end of the $27^{\text {th }}$ vertebra and both ends of the $28^{\text {th }}$ vertebra. The first sacral vertebra $\left(25^{\text {th }}\right.$ 

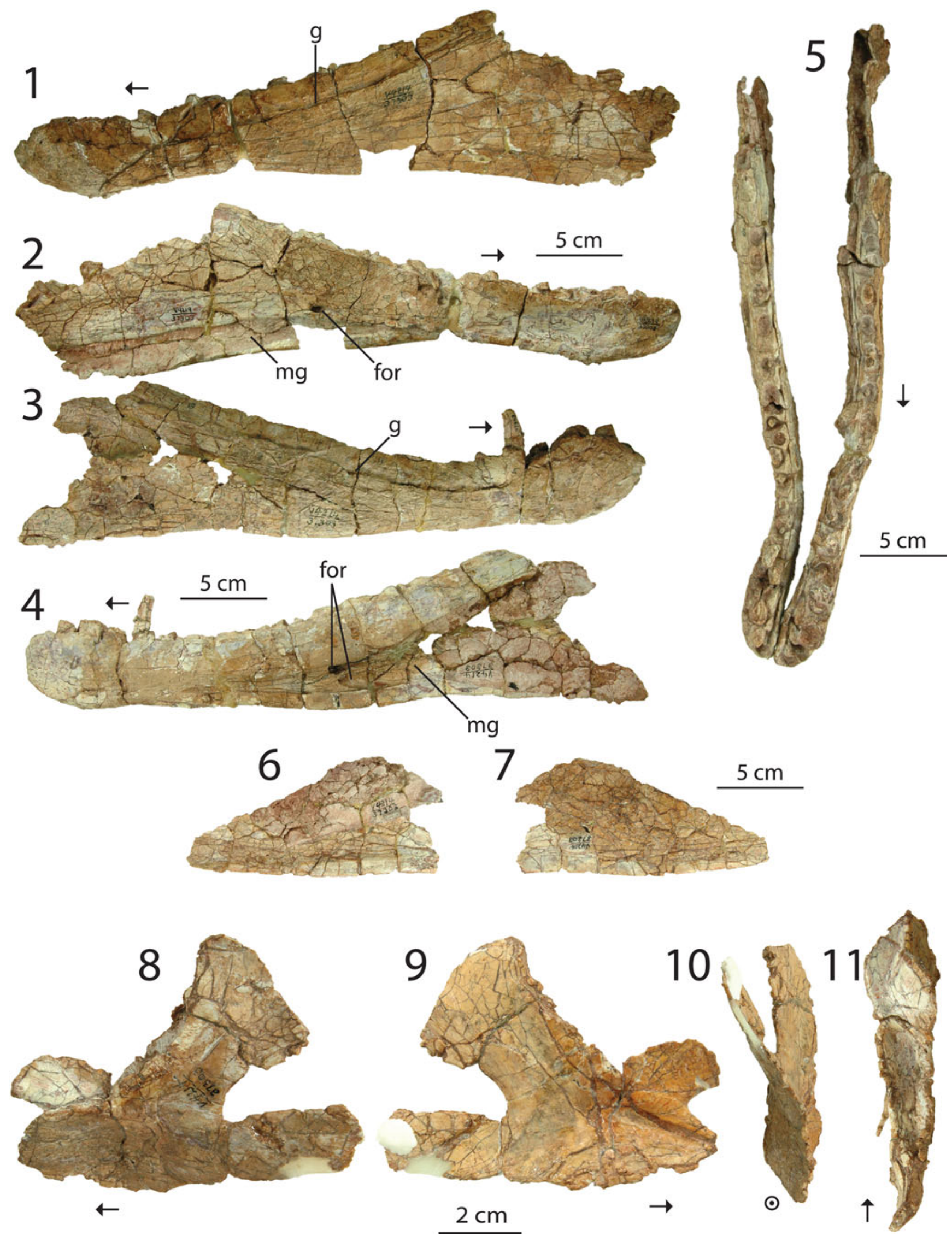

Figure 27. Dilophosaurus wetherilli paratype specimen (UCMP 37303): $(\mathbf{1}, \mathbf{2}, \mathbf{5})$ left dentary, $(\mathbf{3}-\mathbf{5})$ right dentary, $(\mathbf{6}, \mathbf{7})$ right splenial, and $(\mathbf{8}-\mathbf{1 1})$ palatine in $(\mathbf{1}, \mathbf{3}$, $\mathbf{6 , 8})$ lateral, $(\mathbf{2}, \mathbf{4}, \mathbf{7}, \mathbf{9})$ medial, $(\mathbf{5})$ dorsal, $(\mathbf{1 0})$ anterior, and (11) ventral view. Arrows point in anterior direction. for = foramen; $\mathrm{g}=\mathrm{groove} ; \mathrm{mg}=\mathrm{Meckelian}$ groove. 

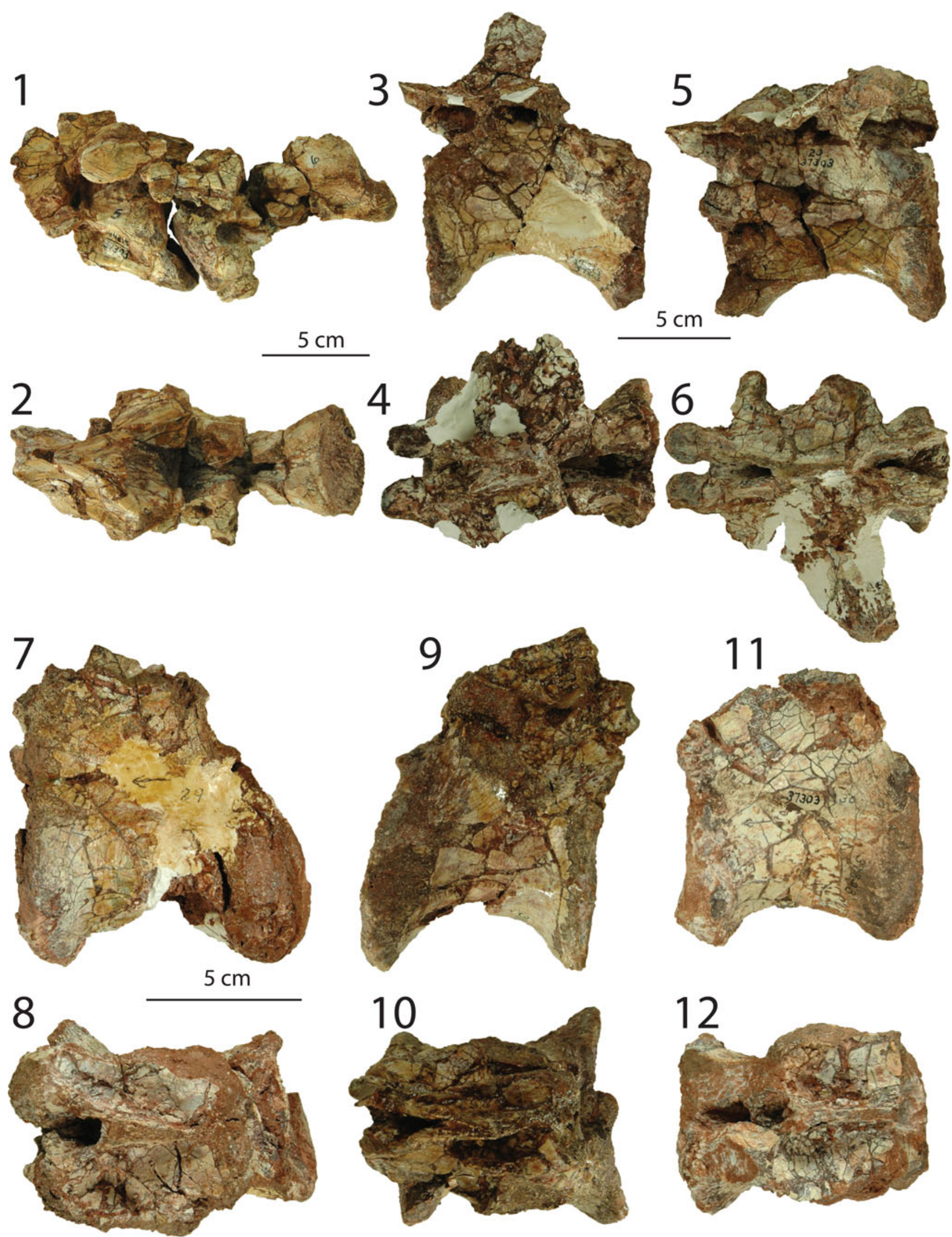

Figure 28. Dilophosaurus wetherilli paratype specimen (UCMP 37303): (1, 2) cervical vertebrae (vertebrae five and six), (3, 4) dorsal vertebra 19, $(\mathbf{5}, \mathbf{6})$ dorsal vertebra $20,(\mathbf{7}, \mathbf{8})$ dorsosacral vertebra, $(\mathbf{9}, \mathbf{1 0})$ caudosacral vertebra, and $(\mathbf{1 1}, \mathbf{1 2})$ anterior caudal vertebra in $(\mathbf{1}, \mathbf{3}, \mathbf{5}, \mathbf{7}, \mathbf{9}, \mathbf{1 1})$ left lateral and $(\mathbf{2}, \mathbf{4}, \mathbf{6}, \mathbf{8}, \mathbf{1 0}, \mathbf{1 2})$ dorsal view. Anterior is to the left. 
vertebra; = dorsosacral of Welles, 1984; Figs. 11.1-11.3, 28.7, $28.8,40.1-40.4$ ) has a tall neural arch that is poorly preserved on the anterior end of the vertebra of the holotype specimen. If the dorsosacral has a rib that contacted the ilium, it was situated anteriorly just below the base of the neural spine.

The $26^{\text {th }}$ vertebra (= the first primordial sacral vertebra; Figs. 11.1-11.3, 40.1-40.4) has a prominent sacral rib. The first sacral rib is not coossified to its articulation surface on the dorsolateral corner of the front of the centrum. The rib is present on the right side in UCMP 37302 and is rounded and subelliptical in lateral view. The rib extends anteriorly and overlaps the back end of the $25^{\text {th }}$ vertebra. The neural spine of the first primordial sacral vertebra leans slightly backwards and is squared distally.

The sacral rib of the $27^{\text {th }}$ vertebra (= the second primordial sacral vertebra; Fig. 11.1-11.3) is much larger than that of the first primordial sacral rib 1, but remains on the anterior end of its centrum and extends anteriorly. In lateral view, the rib of the second primordial sacral vertebra is anteroposteriorly elongate and round, and reaches forward to contact the back of the first primordial sacral rib. The second primordial sacral rib may be coossified to its centrum, but the vertebra is crushed and difficult to interpret in the holotype. The neural arches of primordial sacral vertebrae are missing in the holotype specimen, but the scars on the medial surface of the left ilium suggest that each had a transverse process that touched the ilium above the sacral ribs.

The $28^{\text {th }}$ vertebra (= caudosacral of Welles, 1984; Figs. $11.1-11.3,28.9,28.10)$ is robust and, unlike the prior three centra, the posterior end of the centrum is wider than the anterior end. The centrum is flattened posteroventrally, but lacks the groove found in other caudal vertebrae. The caudosacral does not have a sacral rib, but its transverse process points dorsolaterally and is anteroposteriorly expanded where it contacted the postacetabular process of the ilium. In lateral view, the transverse process is pinched near its base, which is found just behind the longitudinal midpoint of the centrum. The anterior end is shorter than the posterior end, which tapers and extends posteriorly past the back end of the centrum. The neural spine is tall and terminates above the dorsal extend of the inclined transverse processes.

The sacral vertebrae of the referred specimen UCMP 77270 are articulated, but are poorly preserved (Fig. 40.1-40.5). The most anterior vertebra incorporated into the pelvis is consistent with the dorsosacral of the holotype. It does not have a sacral rib and only touches the very front of the ilium along its transverse process beneath the neural spine. The neural arch of the $25^{\text {th }}$ vertebra (the dorsosacral) is found on the anterior end of the vertebra and does not extend posteriorly. The first primordial sacral vertebra (the $26^{\text {th }}$ vertebra) mostly articulates with the front end of the centrum, but also braces the back end of the dorsosacral. The first primordial sacral rib and transverse process nearly touch laterally where they articulate with the ilium. The second primordial sacral vertebra (the $27^{\text {th }}$ vertebra) is missing most of its centrum, but preserves the sacral primordial rib 2 anteriorly. Only the left transverse process remains of the $28^{\text {th }}$ vertebra (the caudosacral), which is very long anteroposteriorly. The transverse process of the caudosacral is tightly coossified to the postacetabular process of the ilium. All four sacral vertebrae are coossified to the ilia wherever they touch, and are closely articulated to one another. A pair of ossified discs are present between the $25^{\text {th }}$ and $26^{\text {th }}$ vertebrae and the $26^{\text {th }}$ and $27^{\text {th }}$ vertebrae in UCMP 77270 (Fig. 40.1, 40.4). These may represent epiphyseal plates or ossified intervertebral discs. The sacral vertebrae are not coossified in any of the specimens, unlike those of coelophysoids (Raath, 1977; Colbert, 1989; Rowe, 1989) and averostrans (Nesbitt and Ezcurra, 2015).

Caudal vertebrae.-The ventral surfaces of the individual caudal centra are marked by a longitudinal midline groove that extends from the anterior end to the posterior end of the centrum, a feature that is present plesiomorphically in dinosaurs. The groove is bounded laterally on both sides by parallel ridges, which are taller posteriorly. The caudal centra are amphicoelous; the articular faces are taller than they are wide in anterior caudal vertebrae, but become circular behind the $40^{\text {th }}$ vertebra $\left(12^{\text {th }}\right.$ caudal; Fig. 12). The ventral margins of the articular faces of each centrum are modified into facets for the haemal arches (=chevrons). The anterior chevron facet is subtriangular and comes to a point ventrally on the midline. The posterior chevron facet is wider and more rounded. In lateral view, the anterior and posterior chevron facets are visible as pronounced sharp-edged corners. Distinct chevron facets are absent by the $50^{\text {th }}$ vertebra ( $22^{\text {nd }}$ caudal).

The caudal neural arches are short, excluding the neural spines. Short hyposphene-hypantrum articulations are present on the $29^{\text {th }}-35^{\text {th }}$ vertebrae. The prezygapophysis is short and robust on the anterior caudal vertebrae, but that of the posterior caudal vertebrae is relatively long and thin, projecting over the centrum. The prezygapophysis articulates with the postzygapophysis of the preceding vertebrae in a tongue-in-groove articulation in caudal vertebrae behind the $45^{\text {th }}$ vertebra, in which the neural spine is absent; the prezygapophysis is located laterally and wraps around and under the postzygapophyses, which are united on a single posteriorly projecting process (Fig. 47.3). By the $38^{\text {th }}$ vertebra, the articular surface of the prezygapophysis points more medially and the postzygapophyseal articular surface points more laterally. The transverse process of more anterior caudal vertebrae is broad and swept backwards past the posterior extent of the postzygapophysis. The transverse process is subhorizontal in posterior view. The transverse processes become relatively less broad and shorter in posterior caudal vertebrae. The transverse process also assumes a more distal position on progressively posterior vertebrae (Fig. 12). As the transverse processes become smaller, a lateral ridge is retained along the length of the neural arch until the transverse processes disappear behind the $45^{\text {th }}$ vertebra $\left(17^{\text {th }}\right.$ caudal $)$.

The neural spines of the first few caudal vertebrae of the holotype specimen are tall, taper upwards, and have subrounded dorsal edges (Figs. 12, 40). The base of the neural spine extends along the entire length of the neural arch. The base of the neural spine of the $34^{\text {th }}$ vertebra $\left(6^{\text {th }}\right.$ caudal $)$ is bifurcated into an anterior process (=anterior spur; Rauhut, 2003) and a more promiment posterior process (Fig. 12). The small anterior process is found on the midline just behind the prezygapophysis and is similar to that found in averostrans (Nesbitt, 2011; Nesbitt and Ezcurra, 2015). Both the anterior process and main neural spine decrease in height progressively down the length of the tail; the anterior spine is absent by the $38^{\text {th }}$ vertebra $\left(10^{\text {th }}\right.$ 


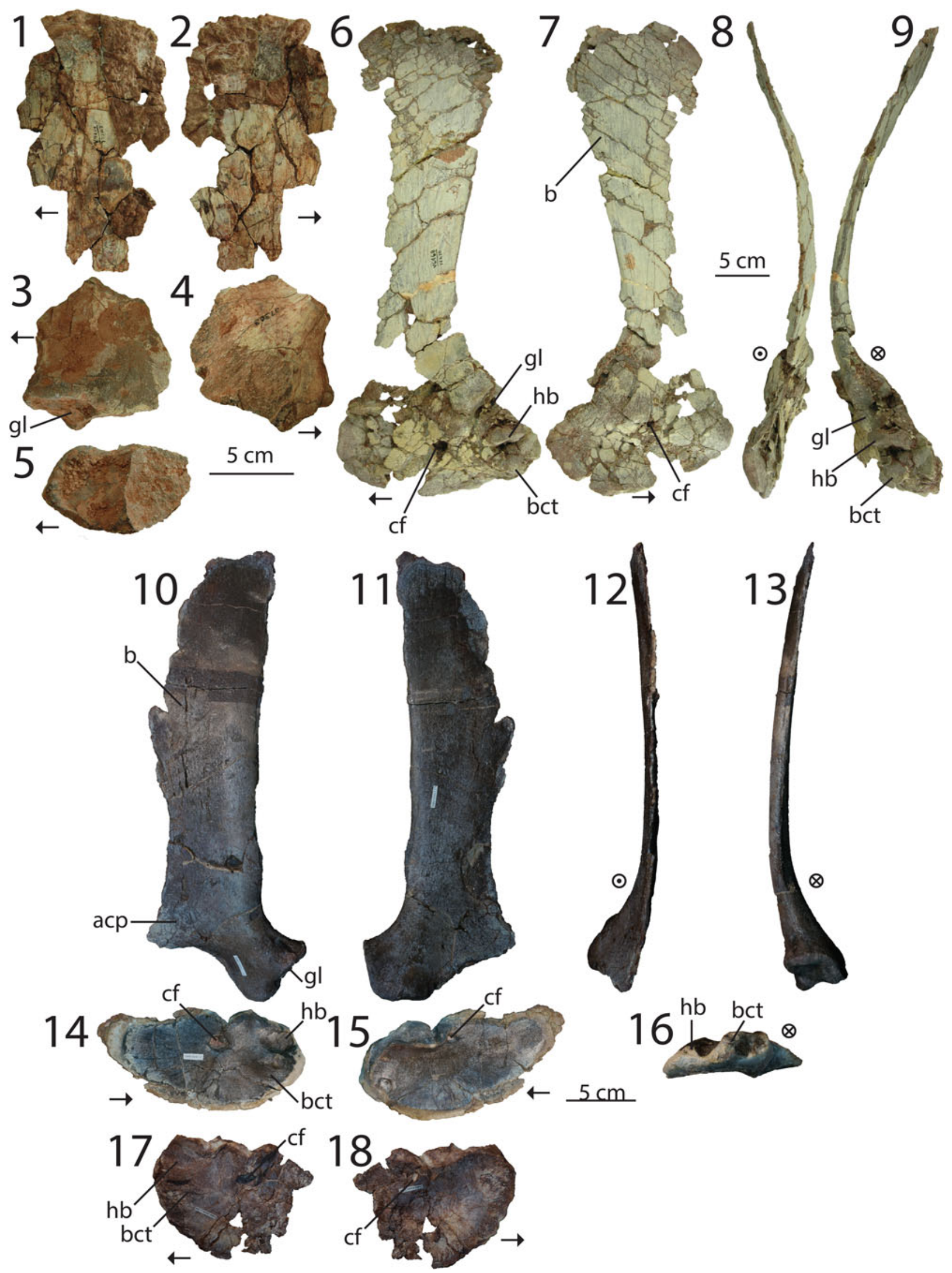

Figure 29. Dilophosaurus wetherilli paratype specimen (UCMP 37303): (1-5) right scapula; Dilophosaurus wetherilli referred specimen (UCMP 77270): (6-9) right scapula; Dilophosaurus wetherilli referred specimen (TMM 43646-1): (10-13) left scapula, (14-16) left coracoid, and $(\mathbf{1 7}, \mathbf{1 8})$ right coracoid. $(\mathbf{1 , 3}, \mathbf{6}, \mathbf{1 0}, \mathbf{1 4}$ 15) Lateral, $(\mathbf{2}, \mathbf{4}, \mathbf{7}, \mathbf{1 1}, \mathbf{1 7}, \mathbf{1 8})$ medial, $(\mathbf{8}, \mathbf{1 2})$ anterior, $(\mathbf{9 , 1 3})$ posterior, $(\mathbf{5})$ proximal, and $(\mathbf{1 6})$ distal view. Arrows point in anterior direction. acp $=$ acromion process; $\mathrm{b}=$ blade; $\mathrm{bct}=$ biceps tubercle; $\mathrm{cf}=$ coracoid foramen; $\mathrm{gl}=$ glenoid; $\mathrm{hb}=$ horizontal buttress. 
caudal), and the larger posterior spine is situated more posteriorly and becomes progressively smaller onto the top of the midline postzygapophyseal process after the $54^{\text {th }}$ vertebra $\left(26^{\text {th }}\right.$ caudal).

The anterior and posterior centrodiapophyseal laminae are present as broad, raised ridges beneath the transverse process of the anterior caudal vertebrae (Fig. 12). The centrodiapophyseal fossa is distinct between these low laminae. The laminae become flush with the lateral side of the neural arch in distal caudal vertebrae; the anterior centrodiapophyseal lamina is not found after vertebra 33 and the posterior centrodiapophyseal lamina is gone after the $36^{\text {th }}$ vertebra (the serial position of these vertebrae is difficult to discern in this area of the holotype specimen because of the missing or reconstructed elements). The spinoprezygapophyseal and spinopostzygapophyseal laminae are well developed and border the spinoprezygapophyseal and spinopostzygapophyseal fossae, respectively. These laminae and fossae diminish and disappear as the neural spine and zygapophyses change in shape down the tail.

Ribs and haemal arches.-The most anterior cervical ribs are poorly preserved (only present in UCMP 77270; Fig. 39), but form simple rod-like structures. Cervical ribs present between the sixth and eighth vertebrae are tetraradiate; the capitulum and tuberculum are situated near one another anteriorly, and the rib extends anteriorly and posterior via separate processes; the longer posterior process makes up the primary shaft of the rib. It tapers distally and extends slightly beyond the posterior margin of its respective vertebra. This is unlike the cervical ribs of coelophysoids (Colbert, 1989; Rowe, 1989), in which the rib shaft extends over the lengths of multiple vertebrae.

The anterior process on the cervical rib of Dilophosaurus wetherilli is bifurcated in posterior cervical vertebrae, forming dorsal and ventral rami (Figs. 40, 46). The ventral ramus is generally more robust and rounded and the dorsal ramus is narrow and pointed. This bifurcation is more pronounced anteriorly. The rib head from a posterior cervical vertebra preserved in UCMP 77270 preserves a single short anterior process that extends over a proximal pneumatic fossa. The capitulum and tuberculum of each cervical rib are connected to one another by a tall lamina that is bordered anteriorly and posteriorly by deep pneumatic fossae. The left ninth cervical rib of UCMP 77270 is pathological; the distal end of the shaft was broken and replaced by a bulbous bony structure (similar to some of the dorsal rib pathologies sometimes found in Tyrannosaurus rex Osborn, 1905; Brochu, 2003).

The trunk ribs are not pneumatized proximally like those of tetanurans (Madsen, 1976; Madsen and Welles, 2000; Brochu, 2003; Sampson and Witmer, 2007). A longitudinal groove extends down the posterior surface of the trunk ribs, but these are present plesiomorphically. The gastral elements are represented by numerous long, thin bones, but their arrangement and association with the rest of the skeleton is unknown (Fig. 11.6-11.8).

The haemal arches (=chevrons) are typical of early saurischians (Fig. 11.4, 11.5). Proximally, each has a transversely divided articular surface that articulates with corresponding surfaces on the bottom of the caudal centra between adjacent vertebrae. The anterior surface of the chevron is subvertical in lateral view and the posterior surface faces posterodorsally. This inclines each chevron posteroventrally when articulated with the caudal vertebrae. The haemal canal is bounded anteriorly by short parasagittal processes on the chevron that extend from the proximal end to a position distal to the haemal canal. The exact length of these processes relative to the chevron is hard to discern because they are mostly broken in the holotype. The midline ventral shaft of each centrum is mediolaterally compressed and especially flattened distally. The distal end is only slightly anteroposteriorly expanded relative to its midshaft, and the entire element is bowed slightly backwards. Contrary to Welles (1984), we found no evidence that the shafts become hatchet-shaped like those of Allosaurus fragilis (Madsen, 1976). Instead, the distal ends of the chevrons widen only slightly anteroposteriorly and retain the plesiomorphic shape for dinosaurs. The shortest chevrons are still fairly straight and relatively thin.

\section{Pectoral girdle}

Scapula.-The scapulae and coracoids are disarticulated in UCMP 37302 (Fig. 13) and TMM 43646-1 (Fig. 29.10-29.16) and are coossified in UCMP 77270 (Fig. 29.6-29.9). The scapula is robust; it is anteroposteriorly expanded at both ends. The left scapula of UCMP 37302 is more complete dorsally, but its anterodorsal edge is crushed, giving it a distinctive kink as described by Welles (1984; Fig. 13). An unusual longitudinal rugose surface on its medial side is probably pathological. The right scapula of UCMP 37302 is complete and uncrushed along the anterodorsal margin, showing that the anterior edge of the scapular blade was smoothly concave.

The dorsal expansion of the scapular blade is asymmetrical; the posterior edge forms a deeper concavity than the anterior edge (Figs. 13.1, 13.10, 29.6, 29.10). The anterior margin of the scapula is weakly concave, unlike the strongly concave anterior margin of the scapula in ceratosaurs and tetanurans (Nesbitt and Ezcurra, 2015). The dorsal margin of the scapula is convex, but its anterior and posterior corners are poorly preserved; the scapula is not 'strap-like' as in early ceratosaurians such as Saltriovenator zanetllai Dal Sasso, Maganuco, and Cau, 2018. The blade is anteroposteriorly thinnest just above the glenoid-acromion region, and it expands anteriorly and posteriorly up the bone. The scapular blade widens ventrally to form the anterior acromion process and the posterior glenoid region. The anteroposteriorly thinnest point along the scapula is just below the midpoint of its dorsoventral length. There is a low, triangular tubercle on the posterior edge of the blade just above the glenoid, possibly where the anterior or posterior arm of the M. scapulohumeralis originates (Burch, 2014; Figs. 13.1, 13.10, 29.6, 29.10).

The acromion process is mediolaterally thin. Its lateral surface is depressed by a semicircular pre-glenoid fossa that spans the distance between the glenoid and the anterior end of the acromion process. The posteroventral corner of the scapula is greatly expanded mediolaterally and anteroposteriorly into the scapular contribution to the glenoid. In ventral view, the glenoid region is divided into three distinct surfaces (Figs. 13.5, 13.14, 29.5). The anterior-most surface is small, triangular, and continuous with the thin acromion process that points forward. The actual glenoid surface of the scapula is the most posterior of the three surfaces. It is subrectangular and faces posterolaterally. The anterior and posterior surfaces are separated by a rhomboidal concave 

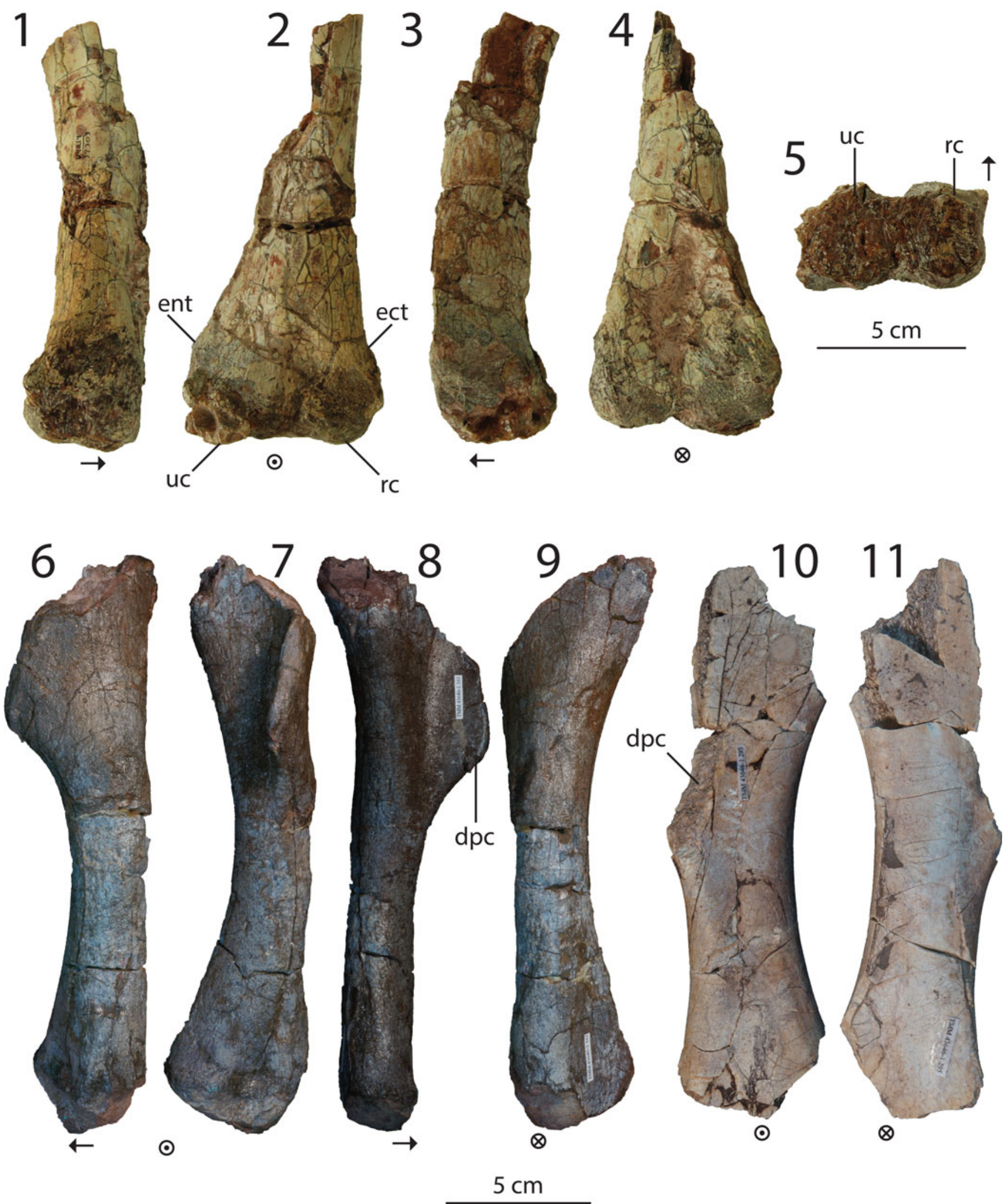

Figure 30. Dilophosaurus wetherilli paratype specimen (UCMP 37303): (1-5) distal end of left humerus; Dilophosaurus wetherilli referred specimen (TMM 43646-1): (6-9) left humerus and $(\mathbf{1 0}, \mathbf{1 1})$ right humerus. $(\mathbf{1}, \mathbf{6})$ Lateral, $(\mathbf{2}, \mathbf{7}, \mathbf{1 0})$ anterior, $(\mathbf{3}, \mathbf{8})$ medial, $(\mathbf{4}, \mathbf{9}, \mathbf{1 1})$ posterior, and $(\mathbf{5})$ distal view. Arrows point in anterior direction. $\mathrm{dpc}=$ deltopectoral $\mathrm{crest}$; ect $=$ ectepicondyle; ent = entepicondyle; $\mathrm{rc}=$ radial condyle; $\mathrm{uc}=$ ulnar condyle. 

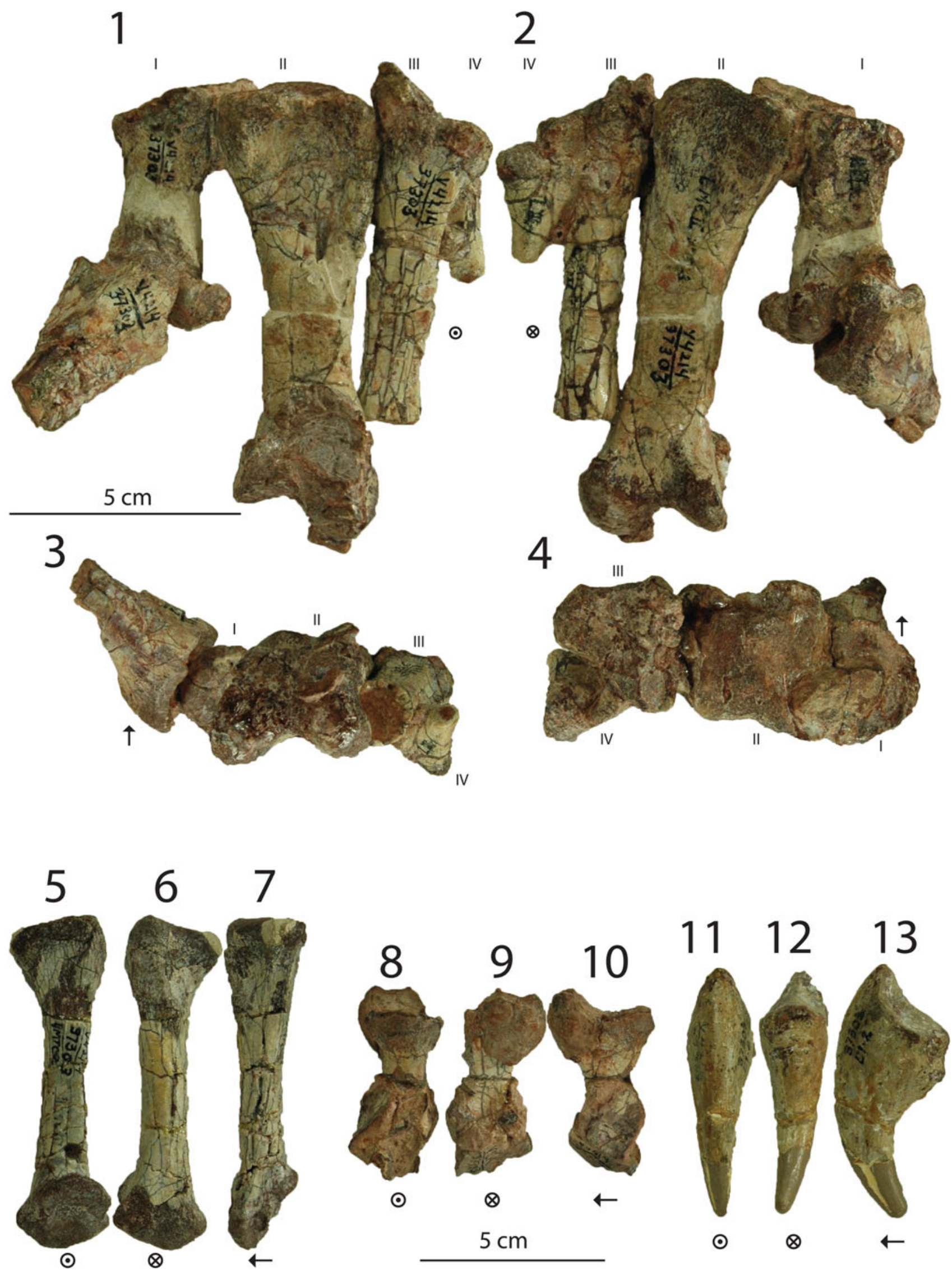

Figure 31. Dilophosaurus wetherilli paratype specimen (UCMP 37303): (1-4) articulated left metacarpus, (5-7) right metacarpal, (8-10) right manual phalanx, (11-13) right manual ungual. $(\mathbf{1}, \mathbf{5}, \mathbf{8}, \mathbf{1 1})$ Dorsal, $(\mathbf{2}, \mathbf{6}, \mathbf{9}, \mathbf{1 2})$ ventral, $(\mathbf{3})$ proximal, $(\mathbf{4})$ distal, and $(\mathbf{7}, \mathbf{1 0}, \mathbf{1 3})$ lateral view. Arrows point in dorsal direction. 

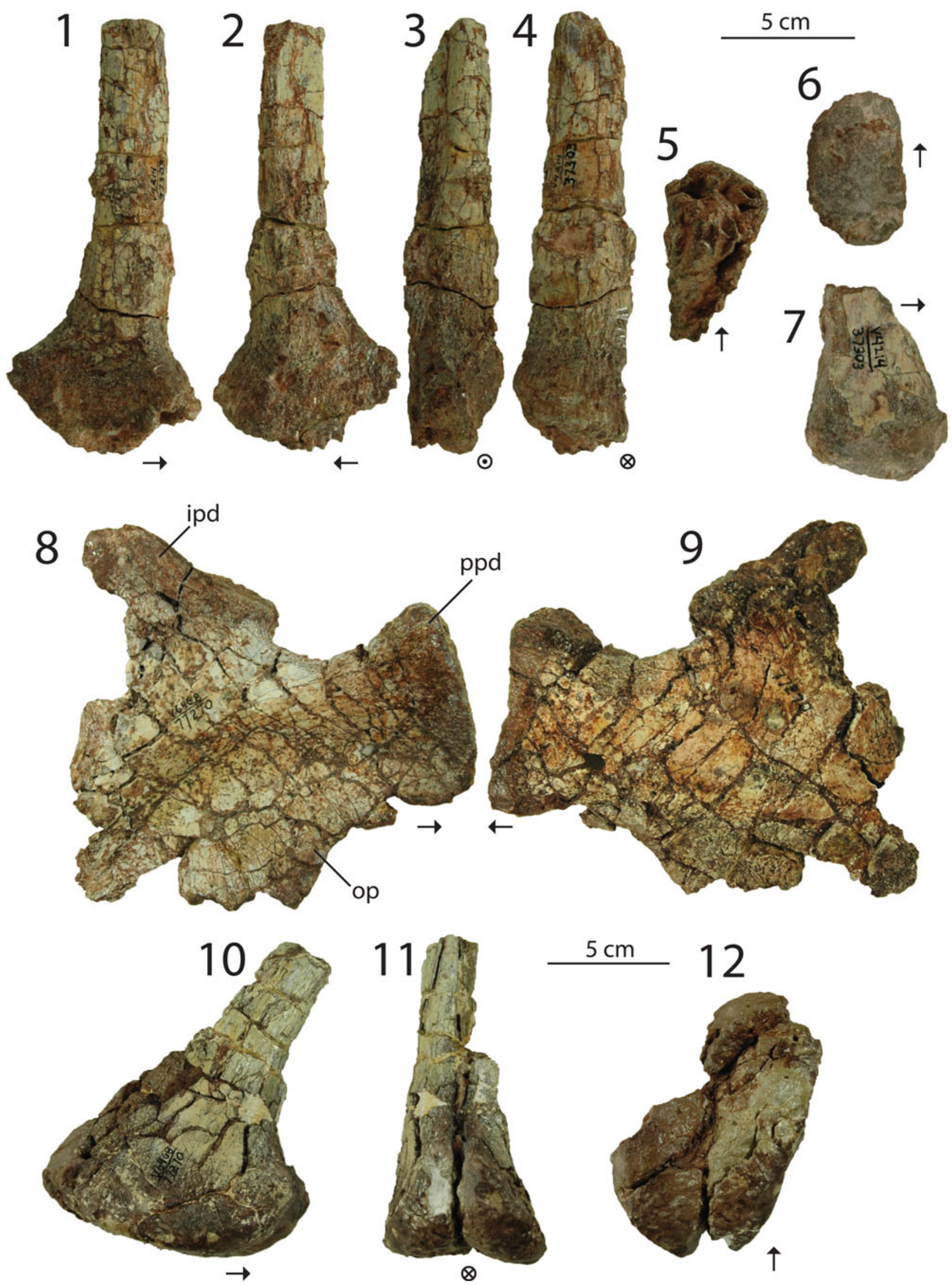

Figure 32. Dilophosaurus wetherilli paratype specimen (UCMP 37303): (1-5) distal end of right ischium and (6, 7) distal end of right pubis; Dilophosaurus wetherilli referred specimen (UCMP 77270): (8,9) proximal end of right ischium and (10-12) distal ends of right and left ischia. $(\mathbf{1 , 7 , 8 , 1 0})$ Lateral, $(\mathbf{2 , 9})$ medial, $(3)$ anterior, $(4, \mathbf{1 1})$ posterior, and $(5,6,12)$ distal view. Arrows point in anterior direction. ipd = iliac pedicle; op = obturator process; ppd = pubic pedicle. 

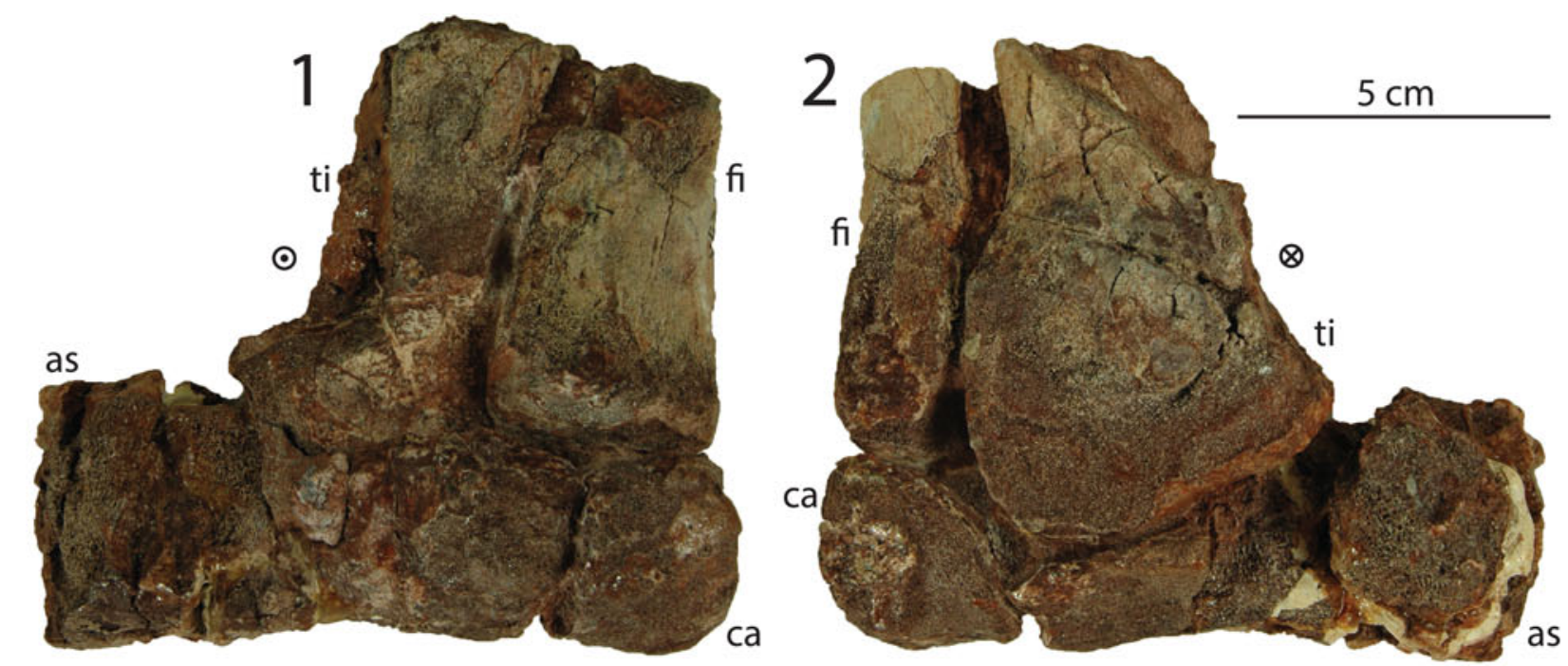

3
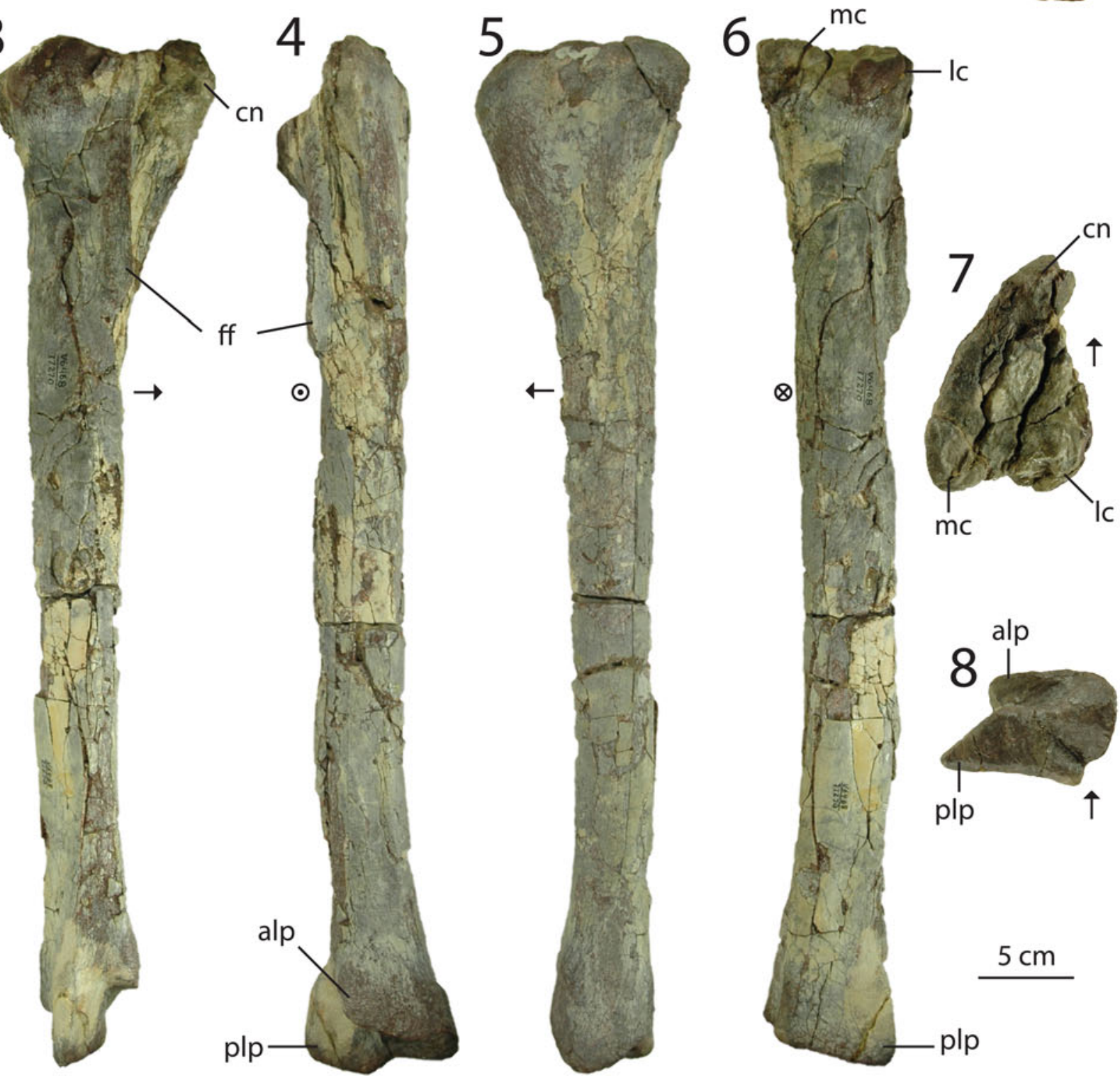
surface that faces ventrally and forms the thickest articular surface for the coracoid.

A lip of bone extends ventrally along the lateral side of the middle ventral surface and contacts a corresponding lip on the coracoid across their articulation to brace the glenoid from the front. The glenoid facet itself is subtrapezoidal; its ventral margin where it contacts the coracoid glenoid facet is straight, but the dorsal corners are round. Welles (1984) mentioned that thick cartilage must have filled the suture between the scapula and coracoid of the holotype specimen, but the two bones actually articulate closely with one another.

Coracoid.-Both coracoids of the holotype of Dilophosaurus wetherilli are missing the thin anterior edge, but seem to be anteroposteriorly longer than they are dorsoventrally high (Fig. 13). The coracoid is subellipitcal in lateral view (Figs. 13, 29.6, 29.14) and lacks the post-glenoid process found in Liliensternus liliensterni (von Huene, 1934), coelophysoids (Raath, 1977; Colbert, 1989; Rowe, 1989), Ceratosaurus nasicornis (Madsen and Welles, 2000), Allosaurus fragilis (Madsen, 1976), and Piatnitzkysaurus floresi (Bonaparte, 1986). The coracoid is curved so that the medial surface is cupped posteriorly but flat anteriorly. The posterodorsal margin of the coracoid has three surfaces that correspond to the three articular surfaces on the posteroventral margin of the scapula. An anterior triangular surface grades into the acromion region.

The coracoid foramen perforates the dorsal half of the bone (Figs. 13, 29.6, 26.7, 29.14, 29.15, 29.17, 29.18). This foramen is circular in lateral view. It passes through the coracoid posterodorsally and exits the medial side as a smaller grooved opening. The middle dorsal surface of the coracoid is thick, rhomboidal, and is convex in lateral view where it articulates with the corresponding concavity on the scapula. The dorsal rim of the glenoid is raised into a small process (subglenoid buttress). The ventral margin of the glenoid facet is not 'lipped' on the coracoid above the coracoid tubercle like it is in sauropodomorphs (Cooper, 1981), Coelophysis bauri (Colbert, 1989), and Syntarsus kayentakatae (Rowe, 1989). The glenoid surface is subtrapezoidal in outline owing to a strong lateral tuberosity that Welles (1984) called the horizontal buttress (Figs. 13.6, 13.8, 29.6, 29.9, 29.14, 29.17). A U-shaped groove (=horizontal groove; Welles, 1984) separates the horizontal buttress from the subparallel coracoid (=biceps) tubercle.

Clavicles (furculum) and sternal plates.-These elements are not preserved in any specimen, but a furculum should be present plesiomorphically in Neotheropoda (Tykoski et al., 2002).

\section{Forelimb}

Humerus.-Correcting for the pathology found in the holotype specimen (Senter and Juengst, 2016; Senter and Sullivan, 2019), the humerus is columnar in anterior view, but is sigmoidal if viewed laterally (unlike the straight lateral profile of Saltriovenator zanellai Dal Sasso, Maganuco, and Cau, 2018; Dal Sasso et al., 2018); the dorsal half bows out anteriorly and the ventral half bows out posteriorly (Figs. 14, 30). The middle of the humerus is subcircular in cross section and is most narrow just below the bottom of the deltopectoral crest. The proximal end is mediolaterally wider than the distal end.

The head of the humerus is robust and convex posteriorly in proximal view (Fig. 14.5, 14.11). A short triangular internal tuberosity (=entotuberosity; Welles, 1984; Burch, 2014) projects posteromedially from the top of the medial side of the head of the humerus. The lateral margin of the head of the humerus is continuous with the deltopectoral crest like that of Piatnitzkysaurus floresi (Bonaparte, 1986) and Saturnalia tupiniquim Langer et al., 1999 (Langer et al., 2007); the deltopectoral crest extends gradually from the proximal end, is slightly rugose at its tallest height, and merges with the shaft near the midlength of the bone. The deltopectoral crest points anterolaterally and bounds the lateral side of a large subtriangular fossa on the anterior surface of the proximal end of the humerus where the M. coracobrachialis inserts (Burch, 2014; Figs. $14.2,14.8,30.7)$. The lateral surface of the deltopectoral crest is flat.

The ectepicondyle is small and is only formed by the rounded lateral corner of the radial condyle (Figs. 14.2, 14.6, $30.2,30.5)$. The entepicondyle is much more prominent and forms a flat oblique surface medial to the ulnar condyle. The olecranon fossa is reduced in size; the radial and ulnar condyles are separated posteriorly by a span of bone that is slightly concave in distal view. A short longitudinal groove separates the distal condyles in anterior view.

Radius.-The radius is expanded at both ends where it articulates with the humerus and carpus (Figs. 15.1-15.18, 41.1-41.5). The proximal and distal outlines are elliptical and circular, respectively. The curved proximomedial surface articulates with the ulna. The distal end has a flattened area medially where it contacts the distal end of the ulna. The radius is curved along its entire length; the anterior margin is convex and the posterior margin is straighter. The radius is thickened two-thirds down the length of the bone. Two low tubercles are found on the posterior surface of the bone; one is near the midshaft and the other is just proximal to that point.

Ulna.-Three structures make up the proximal end of the ulna (e.g., Bonnan, 2003; Figs. 15.11, 15.23, 41.10). A rounded olecranon process is the most proximal component of the ulna. The anteromedial process is long and tapers away from the olecranon, curving slightly laterally. The anterolateral process is very small and forms a rounded knob on the lateral surface of the proximal end of the ulna. The proximal end of the radius fits into the concavity formed between the anteromedial and anterolateral processes. The medial surface of the proximal end of the ulna is flat and subtriangular in shape. A ridge extends from the extremity of the anteromedial process down the shaft and almost to the distal end of the ulna, which is expanded in all directions (Figs. 15.7-15.11, 15.19-15.23, 41.6-41.10). A small, flat area on the anteromedial side of the distal end of the ulna articulates with the distal end of the radius.

Carpus.-The only carpal element preserved in the holotype is consistent with distal carpal 1 in sauropodomorphs and early theropods (Fig. 16.33-16.36). The bone is subelliptical in outline, but is more tapered laterally. The proximal surface is gently convex along its length, and the medial surface is divided into two articular surfaces by a transverse ridge found on the middle of the bone. Two smaller concavities are found along posterolateral edge of the carpal. The proximal rounded surface articulates with the radius or radiale, if present. The two distal surfaces articulate laterally with metacarpal II and distal carpal 2 (distal carpal 2 is a separate element), and articulates 

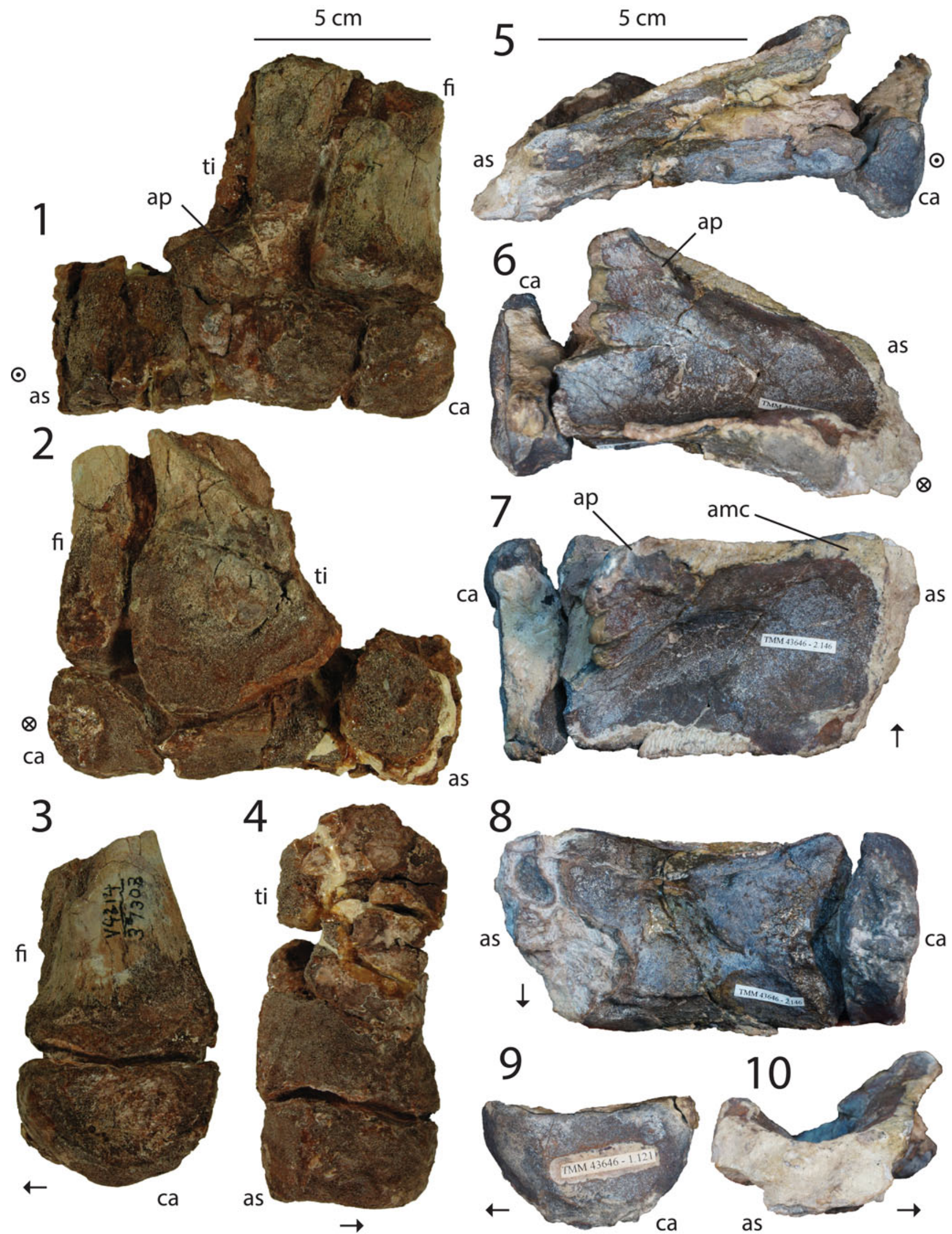

Figure 34. Dilophosaurus wetherilli paratype specimen (UCMP 37303): (1-4) left tibiotarsus; Dilophosaurus wetherilli referred specimen (TMM 43646-1): (510) left astragalocalcaneum. $(\mathbf{1 , 5})$ Anterior, $(\mathbf{2 , 6})$ posterior, $(\mathbf{7})$ proximal, $(\mathbf{4 , 8})$ distal, $(\mathbf{3 , 9})$ lateral, and $(\mathbf{1 0})$ medial view. Arrows point in anterior direction. amc $=$ anteromedial corner; $\mathrm{ap}=$ ascending process; as $=$ astragalus; $\mathrm{ca}=$ calcaneum; $\mathrm{fi}=$ fibula; $\mathrm{ti}=$ tibia . 
medially with the first metacarpal. Although the second and third distal carpals are not present in UCMP 37302, metacarpal I does seem to articulate with the medial side of distal carpal 1 .

Metacarpus.-Four digits make up the manus (Fig. 17.41, 17.42). Digit IV is highly reduced. Metacarpal III is only slightly longer than metacarpal II, and metacarpal IV is longer than metacarpal I. The phalangeal count is 2-3-4-1, and digits I-III terminate in sharp, curved unguals. The metacarpals are arranged linearly in proximal view, except for metacarpal IV, which is tucked underneath metacarpal III.

Metacarpal I is short and stout (Figs. 16.1, 16.5, 31.1, 31.2, 48.1). Its proximal surface is flat and resembles a scalene trapezoid in proximal outline (Figs. 16.29-16.30, 31.4, 48.5). The longer base of the trapezoid contacts metacarpal II. The shaft of metacarpal I narrows slightly before expansion of the asymmetrical distal condyles. Not only does the medial condyle project further distally, it is also more bulbous compared to the thin, disc-like lateral condyle. The large medial distal condyle and small lateral distal condyle create the offset first digit found among saurischian dinosaurs (Figs. 16.31, 16.32, 48.1). These condyles are separated by a vertical groove on the distal surface. A small extensor pit is found behind the lateral distal condyle on the dorsal surface of the bone. Shallow ligament fossae are present on the lateral sides of both distal condyles.

Metacarpal II is subrectangular in proximal view, and the proximal surface is convex (Figs. 16.29, 16.30, 31.4, 48.11). A shallow fossa is present on the proximal end of the dorsal surface of the bone and is bound laterally by a raised edge. The medial surface is flat where the first metacarpal articulates. A slight depression is found laterally where metacarpal III articulates. The shaft of metacarpal II thins distally until it reaches the distal condyles (Figs. 16.9-16.16, 31.1, 31.2, 48.7-48.10). The asymmetry of the distal condyles of metacarpal II is far less pronounced than in metacarpal I, but the medial distal condyle is slightly larger than the lateral distal condyle (Figs. 16.31, $16.32,31.3,31.4,48.12)$. Both condyles expand laterally. A deep, wide extensor pit is found on the dorsal surface of the bone behind the distal condyles. The extensor pit is deepest laterally, and it tapers medially. A tall, curved ridge delineates the proximal margin of the pit, and the pit is continuous with the groove that divides the distal condyles distally. A ligament fossa is only found on the side of the lateral distal condyle.

Metacarpal III is narrow and more gracile than metacarpal II (Figs. 16.17-16.24, 31.1, 31.2, 31.5-31.7, 48.13-48.16). The bone is subtriangular in proximal view (Figs. 16.29, 16.30, $31.4,48.17)$ and the ventrolateral surface is strongly concave where it contacts the fourth metacarpal. The distal condyles of metacarpal III are subsymmetrical in size and shape (Figs. $16.31,16.32,48.18$ ) and an extensor pit is found behind them on the dorsal surface of the bone. This pit faces dorsolaterally and has a curved lip around its proximomedial margin. The distal condyles lack a vertical groove and are only separated ventrally by a shallow depression visible in distal view. The lateral distal condyle has a shallow ligament fossa, but the medial distal condyle does not.

Metacarpal IV is subcylindrical and the distal end curves slightly ventrally (Fig. 16.25-16.28). Both ends are expanded into rounded knobs; the proximal end is wider than the distal end and it has a short ridge on its lateral side (Fig. 16.29,
16.31). The medial surface of metacarpal IV is flat distally. The fourth metacarpal is absent in tetanurans (Nesbitt and Ezcurra, 2015).

Manual phalanges.-Digit I: The first phalanx of digit I (I-1) is the second-longest phalanx in the manus after phalanx II-1 (Figs. 17.1-17.4, 48.19-48.22). The proximal articular surface is subtrapezoidal and is not divided into lateral and medial halves, but instead has an overhanging dorsomedial process that traverses the groove between the distal condyles of metacarpal I. Most of the articular surface of phalanx I-1 articulates with the much larger lateral distal condyle on the first metacarpal. The deep ventral concavity mentioned by Welles (1984) is a result of the thin-walled hollow bone being crushed inwards and prepared into a smooth surface. The distal condyles of I-1 are symmetrical and pointed in dorsal view. They are divided by a strong vertical distal groove. Both distal condlyes house prominent ligament fossae, and the medial fossa excavates most of the inner surface of the medial distal condyle. The first ungual (I-2) is the largest claw in the manus (Fig. 17.517.8). A vertical ridge separates the proximal articular surface into equal halves. The ridge terminates dorsally in a pointed extensor tubercle. The rounded flexor tubercle offset distally from the proximal margin and marks the beginning of the grooves extending down the lateral and medial sides of the bone. The dorsal curvature approximates one-fourth of the circumference of a circle.

Digit II: Digit II is the longest digit in the manus (Fig. 17.41). As Welles (1984) noted, the proximal end of II-1 is divided into two articular surfaces, and the lateral surface is larger and deeper to accommodate the asymmetry in the distal condyles of metacarpal II. This creates a slight lateral offset to the digit, opposite of the medially directed first digit. The extensor tubercle of II-1 is not as pointed as that of I-1. A short process projects ventrally from the ventromedial corner of the proximal articular surface. The distal end of II-1 is reconstructed with painted plaster. Phalanx II-2 is the third-longest phalanx in the manus (Fig. 17.13-17.16). The proximal surface is asymmetrical such that the medial articular surface is larger than the lateral surface. This proximal asymmetry is opposite to that of II-1. The shaft of phalanx II-2 tapers distally, but remains dorsoventrally tall. The distal end has medial and lateral condyles, each with a ligament fossa that is divided by a vertical groove. The ungual of digit II is not as strongly curved as I-2 (Fig. 17.1717.20). The proximal articular surface is weakly divided into subuqual halves. A small flexor tubercle is found on the ventral surface near the proximal end and the extensor tubercle is pointed above the proximal articular surface. A longitudinal groove is present on the medial side of the right second ungual of the holotype specimen.

Digit III: The first phalanx of digit III curves medially in dorsal view (Fig. 17.21-17.24). Most of the proximal surface is a large, shallow lateral concavity, but the dorsomedial surface is convex. A prominent process projects distally from the ventromedial corner of the proximal surface. A smaller process projects ventrally from the ventrolateral corner. The extensor tubercle of phalanx III-1 is a rugose knob. The phalanx terminates in distal condyles, which are slightly twisted relative to the proximal end. A vertical groove separates the two condyles, and each has a shallow ligament fossa. The proximal end of III-2 

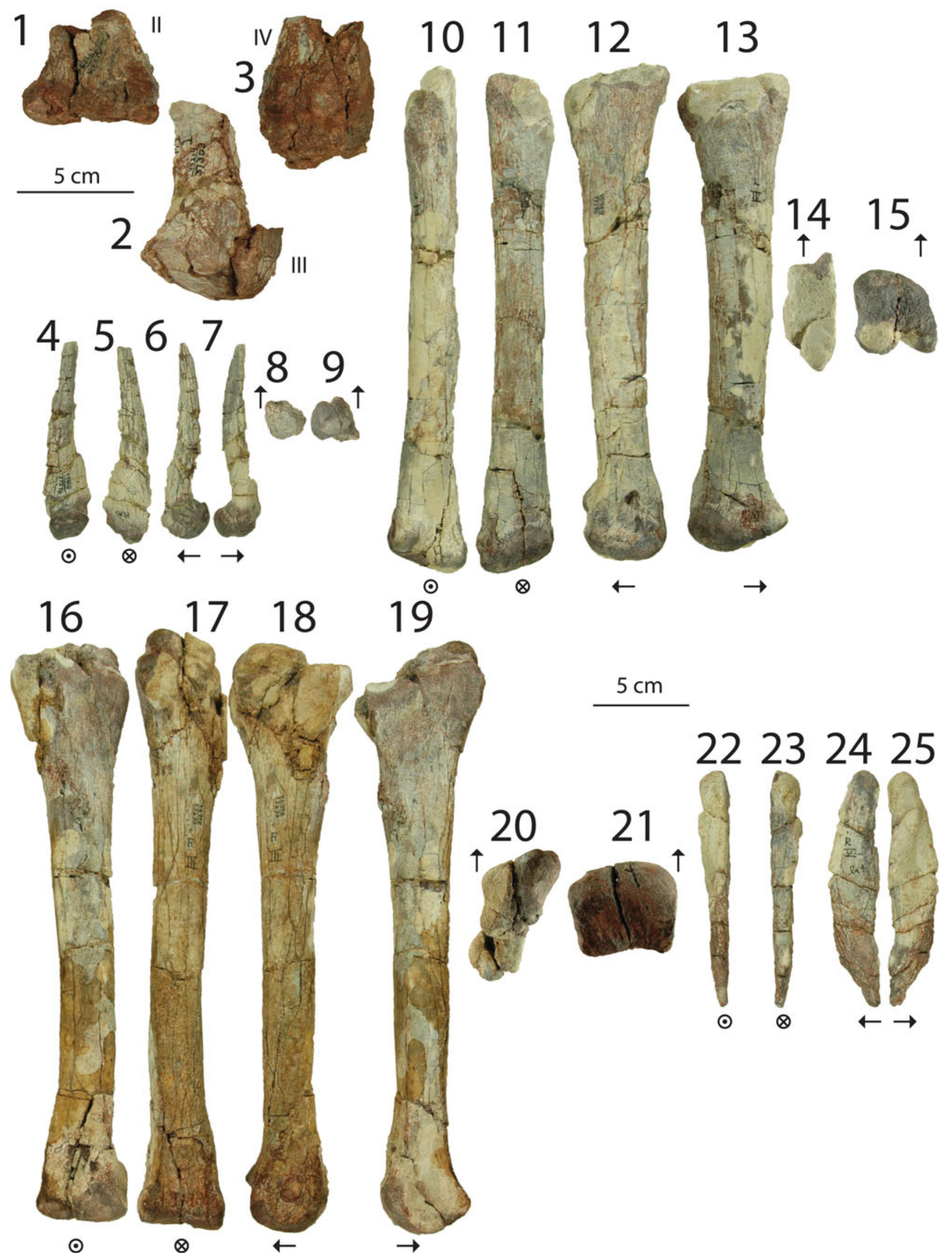

$\odot$

$\otimes$

Figure 35. Dilophosaurus wetherilli paratype specimen (UCMP 37303): (1) metatarsal II, (2) metatarsal III, (3) metatarsal IV; Dilophosaurus wetherilli referred specimen (UCMP 77270): (4-9) right metatarsal I, (10-15) right metatarsal II, (16-21) right metatarsal III, and (22-25) left metatarsal V. (4, 10, 16, 22) Dorsal, (5, 11, 17, 23) ventral, $(6,12,18,24)$ lateral, $(\mathbf{7}, \mathbf{1 3}, 19,25)$ medial, $(\mathbf{8}, \mathbf{1 4}, \mathbf{2 0})$ proximal, and $(\mathbf{9}, 15,21)$ distal view. Arrows point in dorsal direction. 
is crushed (Fig. 17.25-17.28). A strong flexor tubercle projects proximally from the ventral margin of the proximal surface, and the extensor tubercle is equally prominent. The distal end of III-2 resembles that of II-1, but is not twisted relative to the proximal end. Phalanx III-3 is not as robust as the previous two phalanges of the third digit (Fig. 17.29-17.32). The proximal surface is divided into a larger, deeper lateral surface and a smaller, medial surface by a vertical ridge that extends between the low flexor tubercle and pointed extensor tubercle. The distal end does not differ from that of the previous phalanx. The ungual on digit III is curved (Fig. 17.33-17.36). The proximal end has two articular surfaces on either side of a low ridge. The extensor tubercle is pointed and rounded, and resembles that of I-2. Collateral grooves terminate at the tip of the ungual.

Digit IV: The single phalanx is a small peg-like non-ungual phalanx (Fig. 17.17-17.40). Both ends are expanded; the proximal end is wider and slightly concave where it articulates with metacarpal IV. The distal margin of IV-1 is convex.

\section{Pelvic girdle}

Ilium.-The preacetabular process and anterodorsal margin of the iliac blade are unknown in the holotype (Fig. 18.1-18.7). The dorsal-most portion of the blade of UCMP 37302 is anteroposteriorly straight and horizontal. The dorsal surface of the blade is kinked directly above the ischial peduncle, forming a $150^{\circ}$ angle with the anterior portion of the blade. This feature is similar that found in megalosaurids (Benson, 2010; Carrano et al., 2012). A long fossa is present along most of the lateral surface of the blade that accommodated extensive thigh and tail musculature (Hutchinson and Gatesy, 2000; Hutchinson, 2001; Persons IV and Currie, 2011; Schachner et al., 2011).

The preacetabular process of the ilium is squared anteriorly in the complete ilia of TMM 43646-1 (Fig. 49.1, 49.2, 49.5, 49.6); its anteroventral corner forms a right angle and its anterodorsal corner is rounded. The preacetabular process is shorter than the postacetabular process. The bottom of the preacetabular process is straight in front of the pubic peduncle. The preacetabular process is flat laterally.

The postacetabular process of the ilium is relatively long compared to the preacetabular process, but it is not as long and tapered as that of Coelophysis bauri (Colbert, 1989; Figs. 18.1-18.4, 49.1, 49.2, 49.5-49.8). The posterior margin of the postacetabular process is straight and lacks the notch found in coleophysoids (Raath, 1977; Colbert, 1989; Rowe, 1989). The postacetabular process expands mediolaterally in posterior view to form a broad brevis fossa between it and the posteromedial surface of the ischial peduncle (Fig. 18.5), similar to that of the coelophysoids Coelophysis bauri and Syntarsus rhodesiensis (Raath, 1977; Colbert, 1989). The brevis fossa is confined laterally and medially by ridges forming the ventral margin of the postacetabular process that diverge posteriorly, causing the brevis fossa to expand and open backwards. The brevis fossa is walled laterally by a longitudinal ridge of bone that Welles called the 'spine' (1984, p. 133). That ridge extends anteroventrally towards the acetabulum in lateral view and meets two other ridges coming from the ischial peduncle and supraacetabular crest in the posterodorsal corner of the acetabulum. The medial edge of the brevis fossa is confined by a ridge that forms the ventromedial surface of the postacetabular processes that ultimately extends down the back of the ischial peduncle.

The supraacetabular crest projects from the lateral side of the pubic peduncle (Figs. 18.1, 18.8, 49.1, 49.5, 49.7). In ventral view, the supraacetabular crest projects posterolaterally and its lateral rim curves back in towards the midline before joining the 'spine' of the postacetabular process and a short vertical ridge on the lateral surface of the ischial peduncle (Figs. 18.4, 49.4). The anterior half of the supraacetabular crest extends forwards from near the distal end of the pubic penduncle, projecting back ventrolaterally over the dorsal margin of the acetabulum to form a smooth ventral concavity. This is in contrast to the supraacetabular crest of Liliensternus liliensterni (von Huene, 1934) and coelophysoids (Raath, 1977; Colbert, 1989; Rowe, 1989), which projects ventrally.

Both the pubic and ischial peduncles project downwards and outwards away from the supraacetabular crest, forming an open margin at the top of the acetabulum. Both peduncles have flat surfaces that point laterally and slightly inwards to form the inner surface of the acetabulum. The flat anterolateral surface of the ischial peduncle forms the dorsal half of the antitrochanter at the back of the acetabulum. The pubic peduncle is slightly anteroposteriorly expanded at its distal end and it is subtriangular in distal outline. The ischial peduncle is subtrapezoidal in distal view (Figs. 18.4, 49.4). The posterior margin of the ischial peduncle is concave distally in lateral view (=posterior notch; Welles, 1984).

The medial surface of the ilium is flat (Figs. 18.2, 49.2, 49.6, 49.8). The ilia are not coossified to the transverse processes and ribs of the sacral vertebrae in specimens other than UCMP 77270 (Fig. 18.9-18.11). The medial surface of the blade is scarred by radiating ridges, showing that the most-posterior vertebra to contact the pelvis was located at a point on the side of the blade just behind the back of the ischial peduncle (best seen in UCMP 37302, Fig. 18.2 and TMM 43646-1, Fig. 49.2, 49.6). The medial margin of the blade above the articular facets for the sacral vertebrae is slightly concave along its length. A pocket is formed in medial view between the back end of the blade and the posteromedial edge of the postacetabular process above the brevis fossa.

Pubis.-The proximal end of the pubis is coossified to the pubic peduncle of the left ilium in UCMP 77270 (Fig. 18.8, $18.9,18.11)$, but the pubis is isolated in TMM 43646-1 (Fig. 50.8-50.11). The suture between the pubis and the ilium of UCMP 77270 is obliterated laterally, but is visible medially. The anterodorsal margin of the pubis is slightly convex (Figs. 50.8, 50.9, 61.3). The thin obturator plate extends posteroventrally from the pedicles that articulate with the ilium and ischium. An oblong, ovoid obturator foramen perforates the obturator plate and it is surrounded laterally by a fossa that is continuous with the posterior surface of the pubic apron (Fig. 50.8, 50.9). Dilophosaurus wetherilli lacks the second 'pubic fenestra' found in the coelophysoids Syntarsus rhodesiensis (Raath, 1977), Segisaurus halli Camp, 1936 (Carrano et al., 2005), and Gojirasaurus quayi Carpenter, 1997.

The shaft of the pubis is straight (Figs. 19.7-19.14, 50.850.11). The distal end of the pubis is slightly expanded anteroposteriorly and forms a triangle in distal view (Fig. 19.15, 19.16). The triangle formed at the distal end of the pubis tapers 


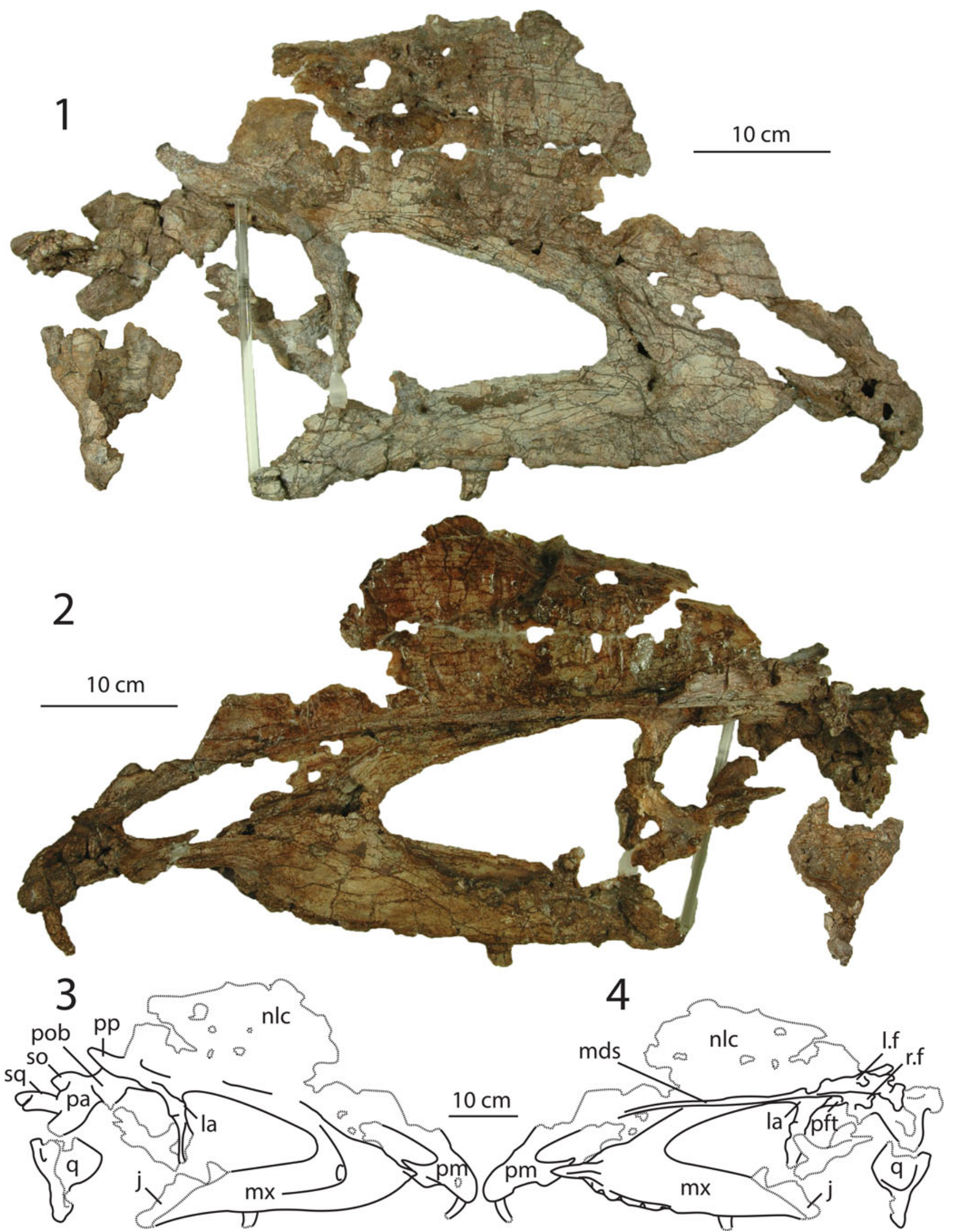

Figure 36. Dilophosaurus wetherilli referred specimen (UCMP 77270): (1-4) articulated skull (mostly right side) in $(\mathbf{1}, \mathbf{3})$ lateral and $(\mathbf{2}, \mathbf{4})$ medial view. $\mathrm{j}=$ jugal; l.f = left frontal; la = lacrimal; $\mathrm{mx}=$ maxilla; $\mathrm{nlc}=$ nasolacrimal crest; $\mathrm{mds}=$ midline suture; $\mathrm{q}=$ quadrate; $\mathrm{pa}=$ parietal; $\mathrm{pft}=$ prefrontal; $\mathrm{pm}=$ premaxilla; pob = postorbital; $\mathrm{pp}=$ posterior process; r.f $=$ right frontal; so = supraoccipital; $\mathrm{sq}=$ squamosal. 

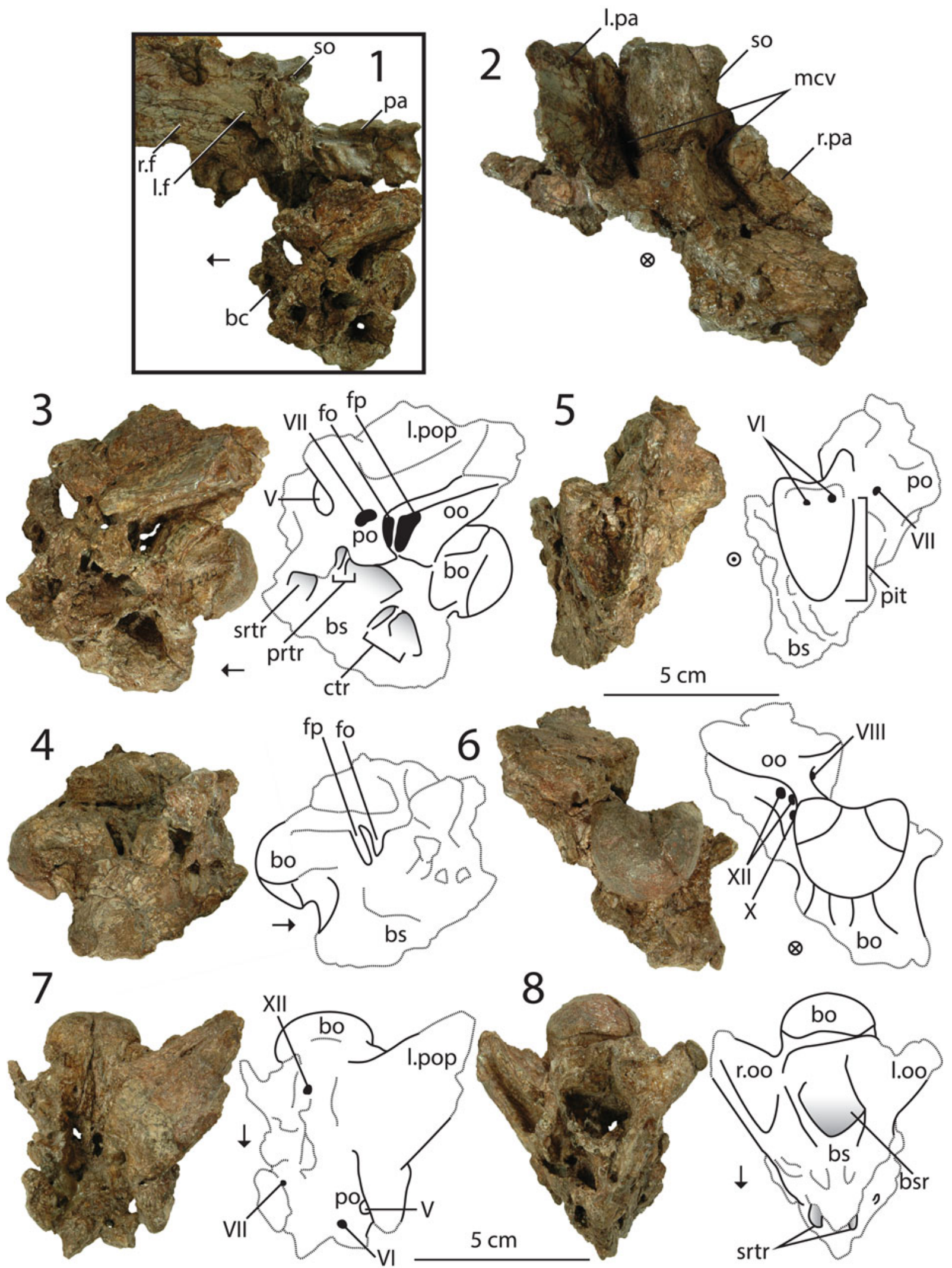

Figure 37. Dilophosaurus wetherilli referred specimen (UCMP 77270): $(\mathbf{1}, \mathbf{2})$ braincase and skull roof and $(\mathbf{3 - 8})$ isolated braincase in $(\mathbf{1}, \mathbf{3})$ left lateral, $(\mathbf{4})$ right lateral, (5) anterior, $\mathbf{( 2 , 6 )}$ posterior, (7) dorsal, and (8) ventral view $(\mathrm{H})$. Arrows point in anterior direction. bc = braincase; bo = basioccipital; bs = basisphenoid; bsr = basisphenoid recess; $\mathrm{ctr}=$ caudal tympanic recess; fo = foramen ovale; $\mathrm{fp}=$ fenestra pseudorotunda; $1 . \mathrm{f}=$ left frontal; $1 . \mathrm{pa}=$ left parietal; $1.00=$ left otooccipital; 1. pop $=$ left paroccipital process of the opisthotic $; \mathrm{mcv}=$ foramen for the middle cerebral vein; oo = otooccipital; $\mathrm{pa}=$ parietal; pit $=$ pituitary fossa; po = prootic; prtr $=$ prootic division of the rostral tympanic recess; $r . f=$ right frontal; $r$.pa $=$ right parietal; r.oo $=$ right otooccipital; so $=$ supraoccipital; srtr $=$ subotic division of the rostral tympanic recess; $\mathrm{V}=$ opening for the trigeminal nerve; $\mathrm{VI}=$ foramen or canal for the abducens nerve; $\mathrm{VII}=$ foramen or canal for the facial nerve; VIII = foramen or canal for the vestibochochlear nerve; $\mathrm{X}=$ foramen or canal for the vagus nerve; $\mathrm{XII}=$ foramen or canal for the hypoglossal nerve. 
posteriorly. The pubes contact one another distally along a flat surface on the midline, but are not coossified to one another in any specimens of Dilophosaurus. The pubic aprons are broken on both bones, but would have projected posteromedially from each side to contact one another along the midline.

Ischium.-The proximal margin of the ischium comprises the ilial, pubic pedicles, and the obturator process (Figs. 19.1$19.6,32.8,32.9,50.7)$. The obturator processes of the ischia are coossified to one another along the entire anterior margin of ischium from beneath the pubic pedicles to the proximal part of the shafts, which are not coossified to one another proximally (best seen in UCMP 37302; Fig. 19.1-19.3). The ilial pedicle is anteroposteriorly longer than the pubic pedicle, and it is subelliptical in proximal outline. Proximally, the ilial pedicle is divided into posterior and anterior surfaces by an anteromedial ridge (Fig. 19.6). The posterior surface articulates with the ilium and the anterior surface forms the ventral half of the antitrochanter behind the acetabulum. A notch extends between the pubic and ilial pedicles on the proximal end of the ischium. The pubic pedicle is subtriangular in proximal view and tapers anteroventrally into the obturator process (Fig. 19.5). A U-shaped notch divides the lobed ventral end of the obturator process from the ischial shafts (Figs. 19.1, 19.2, 50.7), similar to that found in Tachiraptor admirabilis (Langer et al., 2014) and Dracoraptor hanigani (Martill et al., 2016). The anterior edge of the obturator process is divided into two convex edges by a wide notch, the proximal of which is longer than the distal. The posterior margin of the ischium is formed by a ridge that extends from the ilial pedicle down the shaft of the ischium.

In distal outline, the broken shafts of the holotype ischia are subtriangular. The ischiac apron is incomplete medially. The apron is straight where it emanates from the shaft of the ischium. The distal extremity of the ischium is rounded and triangular in outline; the widest portion of the triangle faces anteriorly (Figs. 32.1-32.5, 50.1-50.6). The posterodorsal margin of the distal end of the ischium is straight, but the anteroventral margin is concave. The distal expansion is subtriangular in lateral outline. The lateral surface is convex in distal view and the medial side is flat where the ischia articulate. The distal ends of the ischia are widest posteriorly and taper forwardly, forming a subtriangular distal outline like that of Herrerasaurus ischigualastensis and sauropodomorphs (Novas, 1994; Langer, 2003; Moser, 2004), but unlike that of other theropods and Eoraptor lunensis, in which the cross-section is subelliptical (Carrano et al., 2005; Nebitt, 2011; Sereno, 2012; Nesbitt and Ezcurra, 2015).

\section{Hindlimb}

Femur.-The femur is sigmoidal in anterior and lateral views; the proximal half is concave medially and the distal half is convex laterally in anterior view (Figs. 20.1-20.4, 42.4, 42.8, $51,52.1-52.8)$. The long axes of the proximal and distal ends are twisted $50^{\circ}$ with respect to one another. The three distinct tubera found plesiomorphically in theropods are present on the head of the femur in proximal view: the anterolateral tuber, the anteromedial tuber, and the posteromedial tuber (Nesbitt, 2011; Figs. 20.5, 52.9, 61.8). The anterolateral tuber is a broad surface that forms the anterior curvature of the femoral head. The anteromedial tuber forms the most medial surface of the femoral head that inserts into the acetabulum. This tuber is expanded into a thickened proximodistal ridge in posterior view and is expanded distally to form a 'hook.' A smooth, longitudinal U-shaped sulcus separates the hooked anteromedial tuber from the posteromedial tuber. The posteromedial tuber is best seen in proximal view where its convex curve makes up much of the posterior margin of the femoral head. A straight, transverse groove is partially visible along the proximal surface of the femoral head between the anterolateral and posteromedial tubera (Fig. 52.9); it is curved in Tawa hallae (Nesbitt et al., 2009b), Cryolophosaurus ellioti (Smith et al., 2007), Liliensternus liliensterni (von Huene, 1934), and coelophysoids (Raath, 1977; Colbert, 1989; Rowe, 1989), but Chindesaurus byansmalli Long and Murry, 1995, Allosaurus fragilis (Madsen, 1976), and Ceratosaurus nasicornis (Madsen and Welles, 2000) lack such a groove.

The greater trochanter (=major trochanter: Cooper, 1981; =dorsolateral trochanter or ridge: Nesbitt, 2011; Griffin, 2018) is found on the lateral surface of the femur below the most distal level of the anteromedial tuber on the opposite side of the femoral head (Figs. 20.4, 42.8, 52.8). In lateral view, the greater trochanter is subelliptical in shape and forms a rounded ridge, unlike the tall, sharp ridge found in Liliensternus liliensterni (von Huene, 1934) and Tawa hallae (Nesbitt et al., 2009b) or the smooth area found in averostrans (Nesbitt, 2011; Nesbitt and Ezcurra, 2015).

The proximal end of the anterior trochanter (=lesser trochanter: Rauhut, 2003) is found medial to, but at the same proximodistal level as, the distal end of the greater trochanter. The anterior trochanter is a prominent subelliptical eminence in UCMP 37302 that tapers distally to join the femoral shaft, but expands proximally and is set apart from the top of the femur by a notched groove (Fig. 20.2, 20.3). A conspicuous trochanteric shelf is absent in the holotype, but the groove separating the top of the anterior trochanter from the femur opens medially, constructing a broadly sloping surface. A very low mound projects lateral to the distal extent of the anterior trochanter on the lateral surface of the femoral shaft below the greater trochanter, representing the posterolateral tuber found in many theropods, but only prominent in maniraptorans (Rauhut, 2003). This mound is found in UCMP 37302 (Fig. 20.2) and the referred right femur MNA V160/V161 (Fig. 61.4).

The anterior trochanter of UCMP 77270 is an elongate, raised bar that is pointed proximally and separated from the shaft of the femur by a sharp notch (Fig. 42.6). A long, rugose ridge extends distally from this proximal process and reaches down almost halfway down the femur. Such a ridge is also present on coelophysoid femora with trochanteric shelves (e.g., UCMP 129618/PEFO 21373; Padian, 1986; Rowe, 1989; Griffin, 2018). The proximal projection of the anterior trochanter is triangular in anterior view and is braced from below and on its medial side by a trochanteric shelf. The trochanteric shelf of UCMP 77270 is flat proximally but forms a concave surface once it extends up the front of the proximal projection of the anterior trochanter.

The fourth trochanter (=insertion of M. caudofemoralis, Nesbitt, 2011) of Dilophosaurus wetherilli is long and sigmoidal, extending down the back of the femoral shaft (Figs. 20.1, 20.4, 42.1, 42.3, 42.7, 52.1, 52.3, 52.4, 52.7, 52.8). The middle of the fourth trochanter of the holotype is 


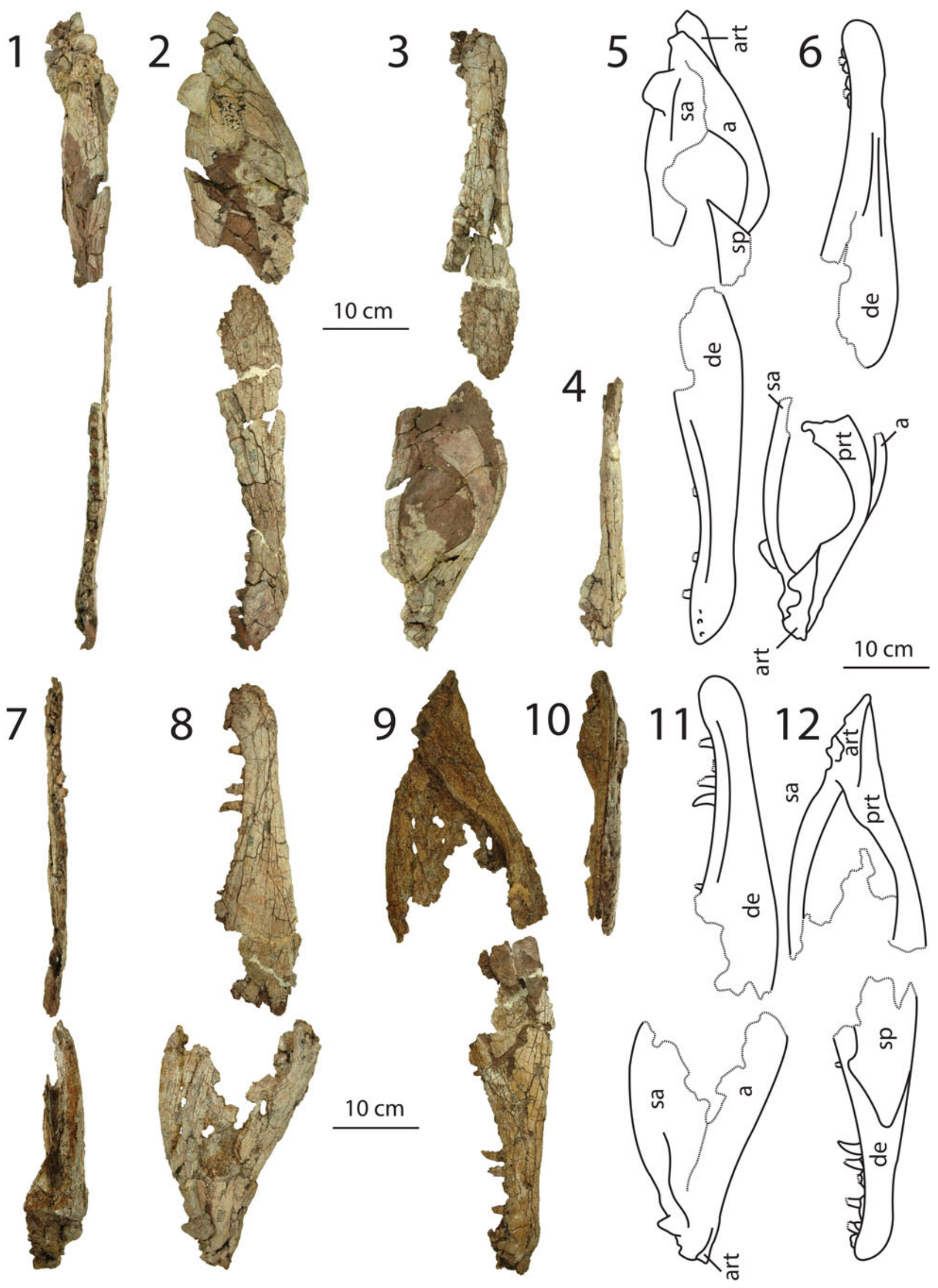

Figure 38. Dilophosaurus wetherilli referred (UCMP 77270): (1-6) left mandible and (7-12) right mandible in $(\mathbf{1}, \mathbf{7})$ dorsal, $(\mathbf{2}, \mathbf{5}, \mathbf{8}, \mathbf{1 1})$ lateral, (3, 6, 9, 12) medial, and $(4, \mathbf{1 0})$ ventral view. $\mathrm{a}=$ angular; art $=$ articular; $\mathrm{de}=$ dentary; $\mathrm{prt}=$ prearticular; $\mathrm{sa}=$ surangular; $\mathrm{sp}=$ splenial . 


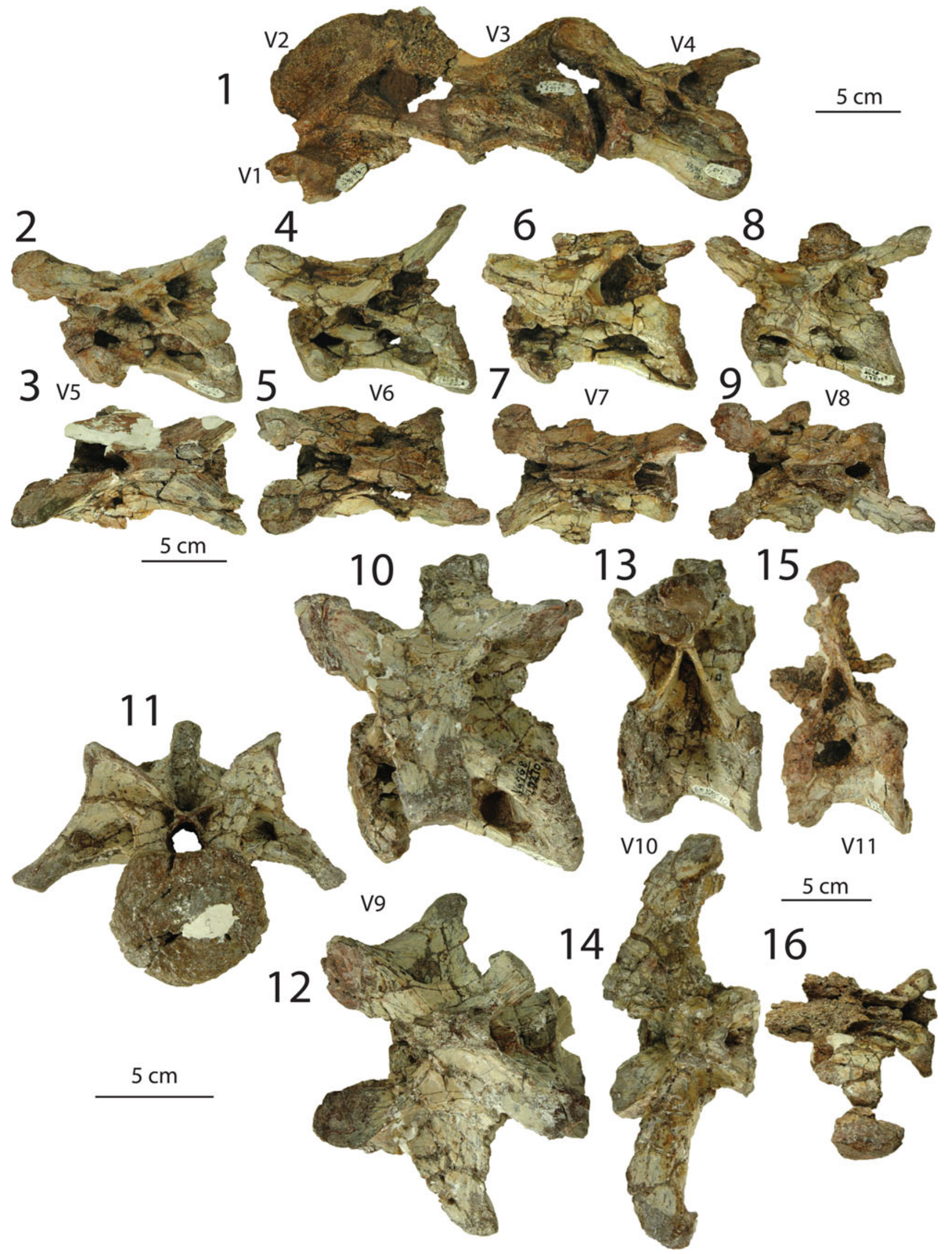

Figure 39. Dilophosaurus wetherilli referred specimen (UCMP 77270): (1-16) cervical vertebrae (first through $\left.11^{\text {th }} ; \mathrm{V} 1-\mathrm{V} 11\right)$ ) in $(\mathbf{1}, \mathbf{2}, \mathbf{4 ,}, \mathbf{6}, \mathbf{8}, \mathbf{1 0}, \mathbf{1 3}, \mathbf{1 5})$ left lateral, $(\mathbf{3}, \mathbf{5}, \mathbf{7}, \mathbf{9}, \mathbf{1 2}, \mathbf{1 4}, \mathbf{1 6})$ dorsal, and $(\mathbf{1 1})$ anterior view. Anterior is to the left. $\mathrm{v}=$ vertebra 


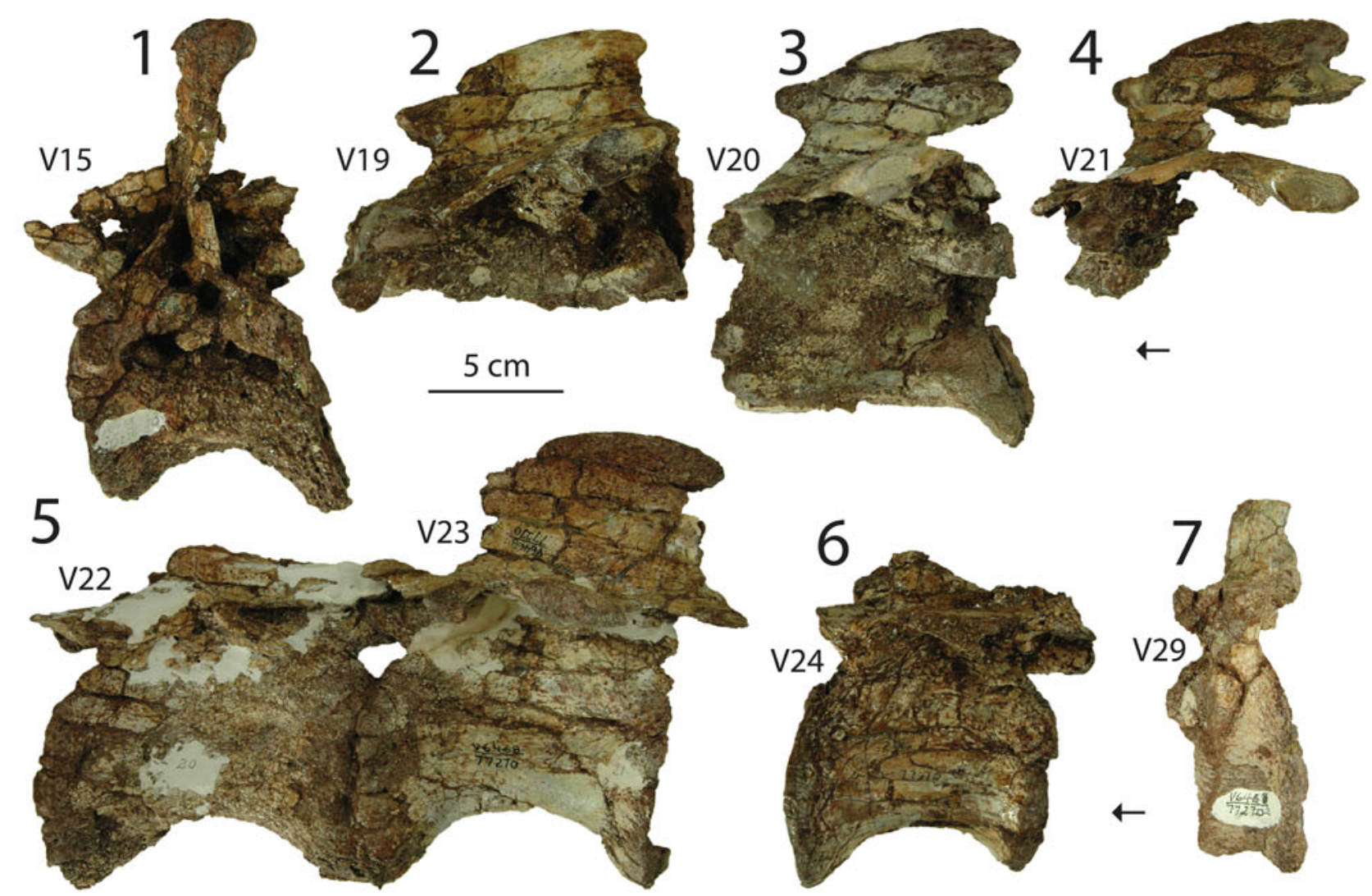

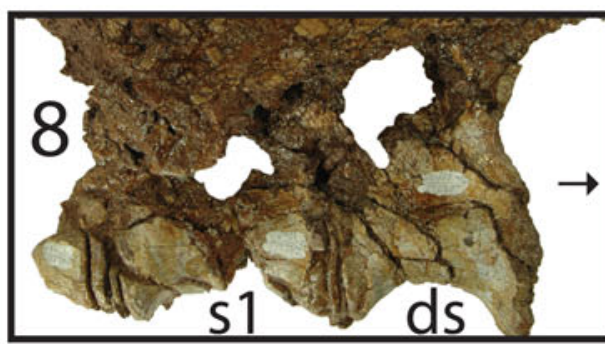

12 osc

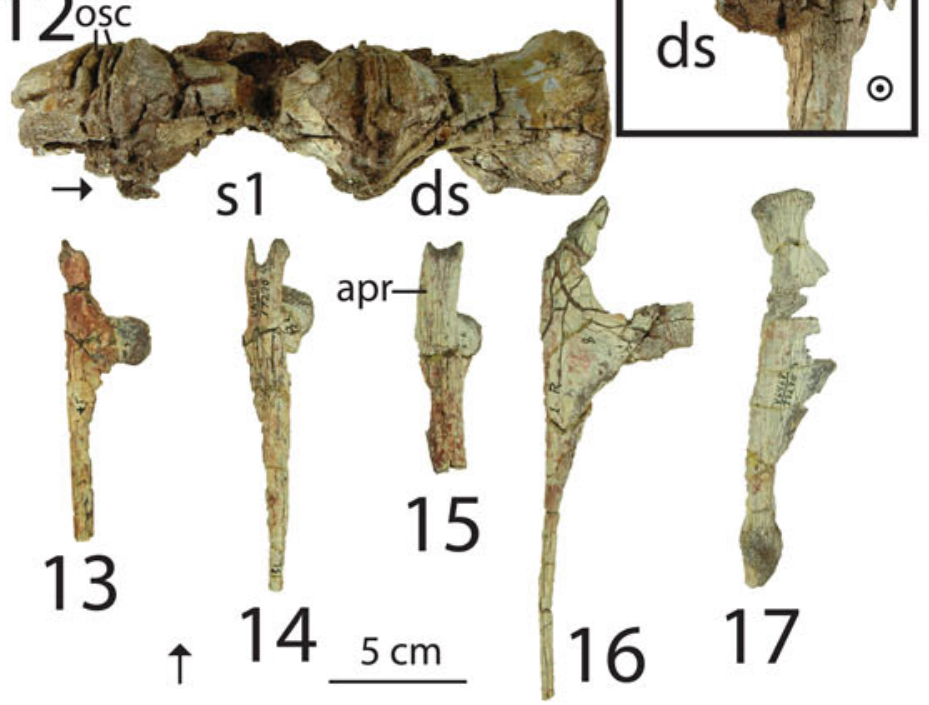

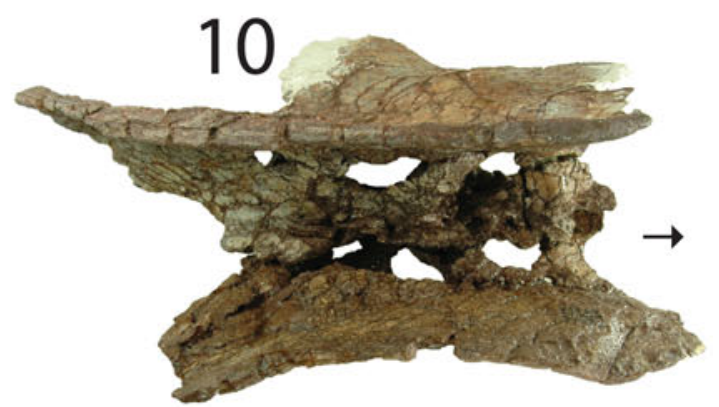

$5 \mathrm{~cm}$

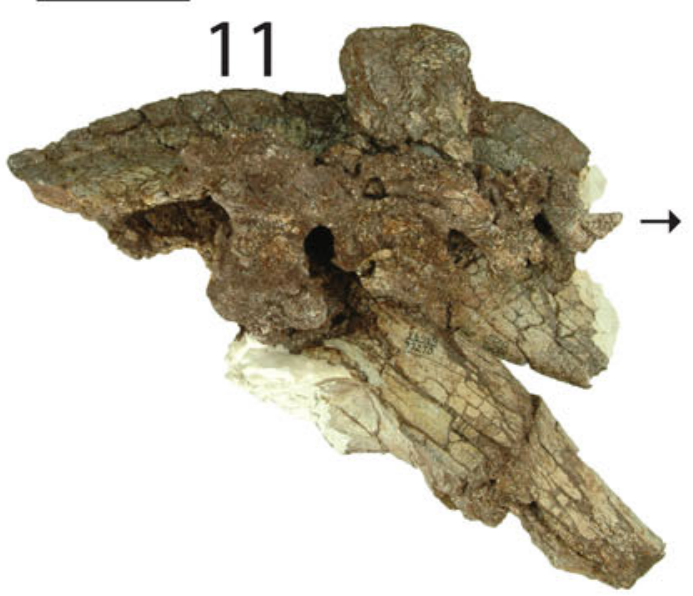

Figure 40. Dilophosaurus wetherilli referred specimen (UCMP 77270): (1-6) trunk vertebrae (vertebrae 15, 19 through 24; V15, V19-V24, V29), (7) anterior caudal vertebra, (8-12) dorsosacral vertebra and first sacral vertebra, (13) right fifth cervical rib (reversed), (14) sixth left cervical rib, (15) left seventh cervical rib, (16) left eight cervical rib, (17) left ninth cervical rib in (1-7, 13-17) left lateral, (8) right lateral, (9) posterior, (10) dorsal, (11) medial, and (12) ventral view. Arrows point in anterior direction. $\mathrm{apr}=$ anterior process; $\mathrm{ds}=$ dorsosacral vertebra; $\mathrm{osc}=\mathrm{ossified}$ cartilage; $\mathrm{s} 1=$ sacral vertebra; $\mathrm{v}=\mathrm{vertebra}$. 


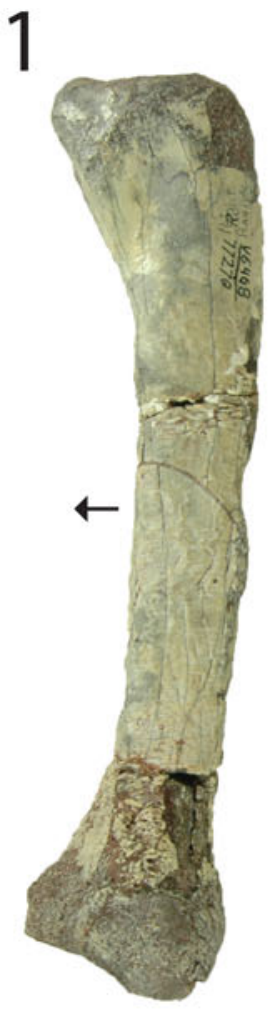

2

$\odot$

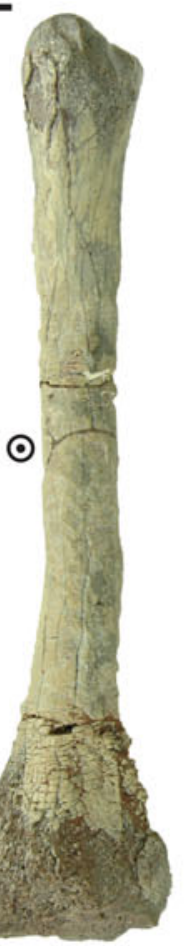

6

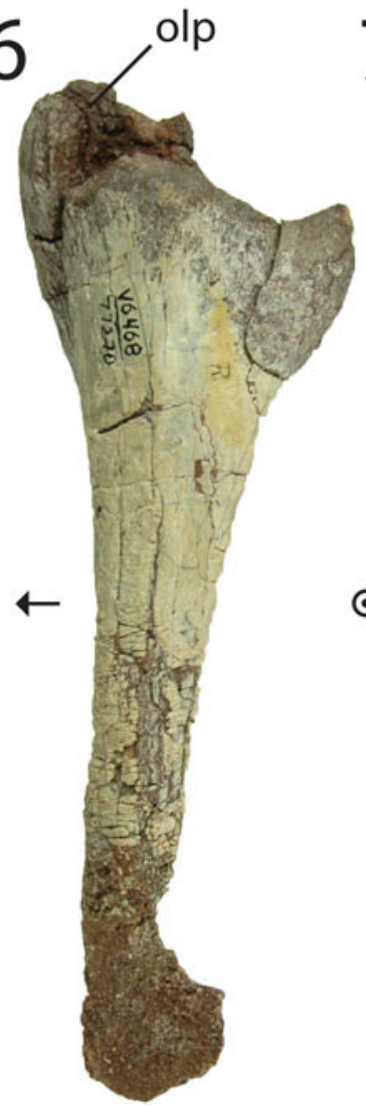

3

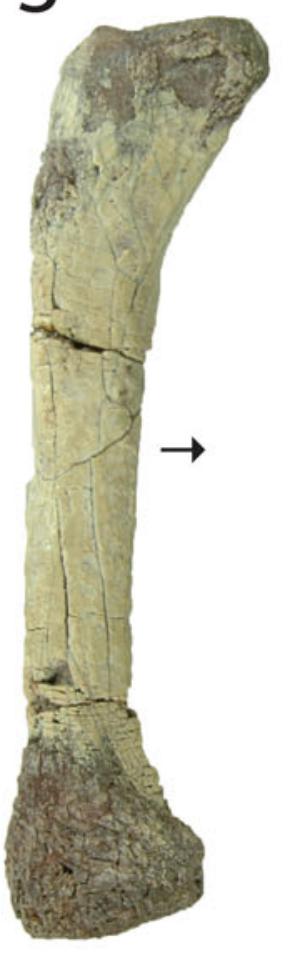

8

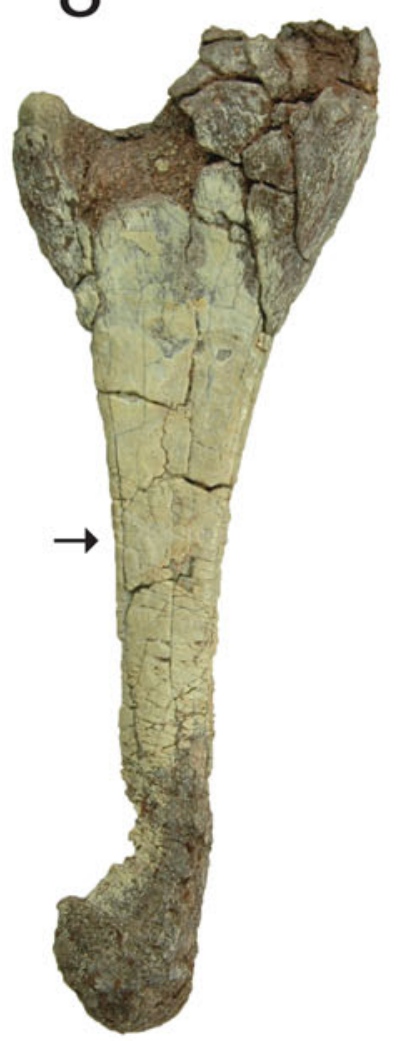

4

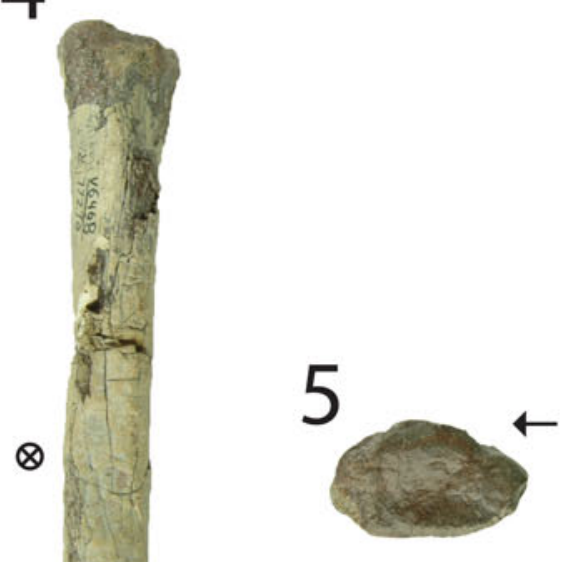

9

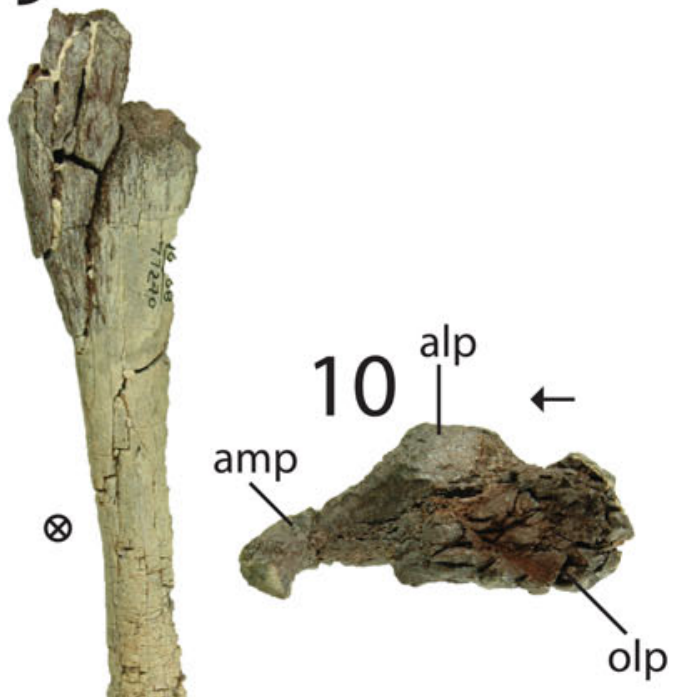

Figure 41. Dilophosaurus wetherilli referred specimen (UCMP 77270): (1-5) right radius and (6-10) right ulna in $(\mathbf{1 , 6})$ lateral, $(\mathbf{2}, \mathbf{7})$ anterior, $(\mathbf{3}, \mathbf{8})$ medial, $(\mathbf{4 , 9})$ posterior, and $(\mathbf{5}, \mathbf{1 0})$ proximal view. Arrows point in anterior direction. alp $=$ anterolateral process; amp $=$ anteromedial process; olp $=$ olecranon process. 


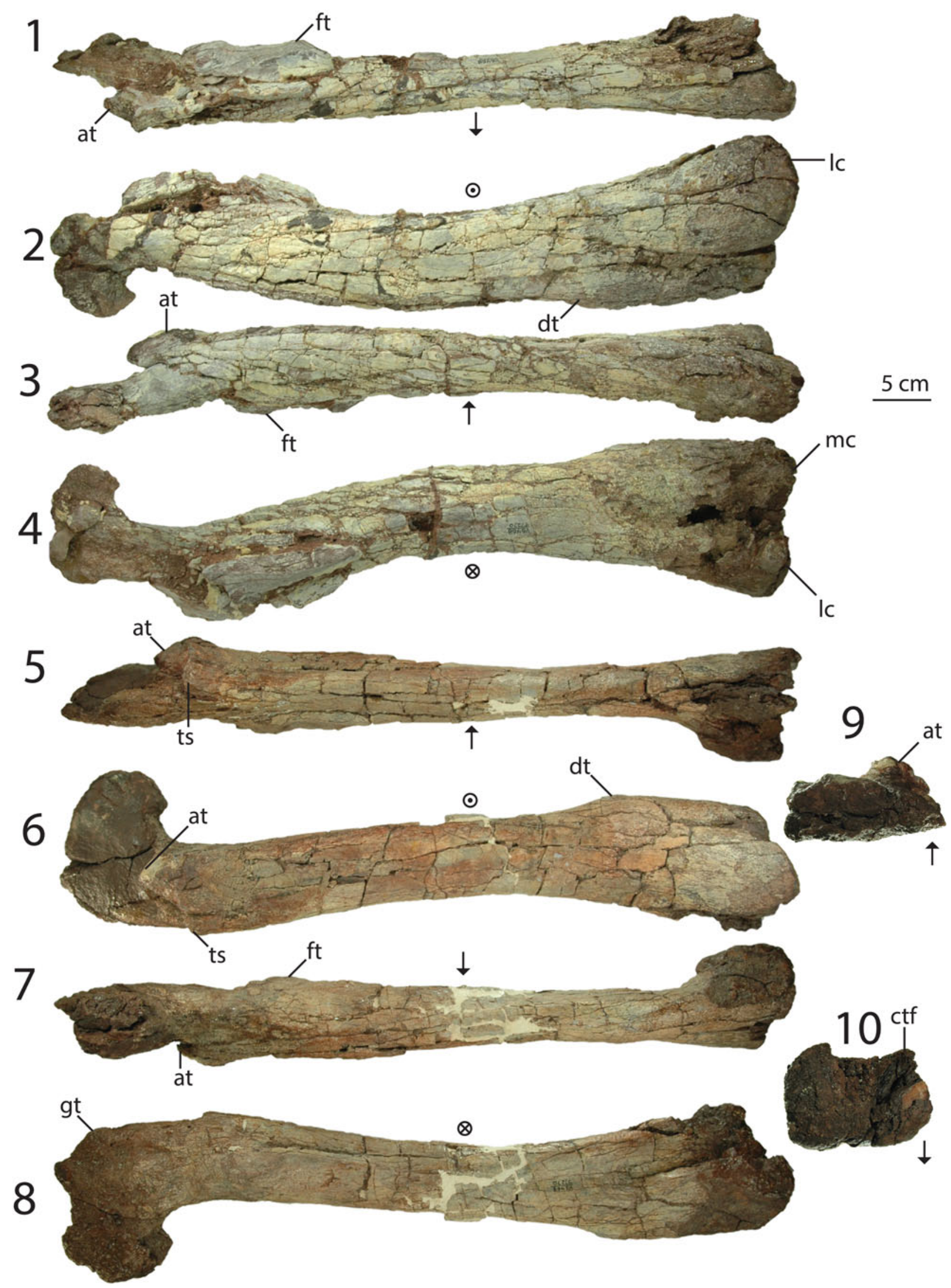

Figure 42. Dilophosaurus wetherilli referred specimen (UCMP 77270): (1-4) left femur and (5-10) right femur in $(\mathbf{1}, \mathbf{5})$ lateral, $(\mathbf{2}, \mathbf{6})$ anterior, $(\mathbf{3}, \mathbf{7})$ medial, $(\mathbf{4}, \mathbf{8})$ posterior, (9) proximal, and (10) distal view. Arrows point in anterior direction. at $=$ anterior trochanter; $\mathrm{ctf}=\mathrm{crista}$ tibiofibularis; $\mathrm{dt}=\mathrm{distal}$ tuberosity; $\mathrm{ft}=\mathrm{fourth}$ trochanter; $\mathrm{gt}=$ greater trochanter; $\mathrm{lc}=$ lateral condyle; $\mathrm{mc}=$ medial condyle; $\mathrm{ts}=$ trochanteric shelf. 

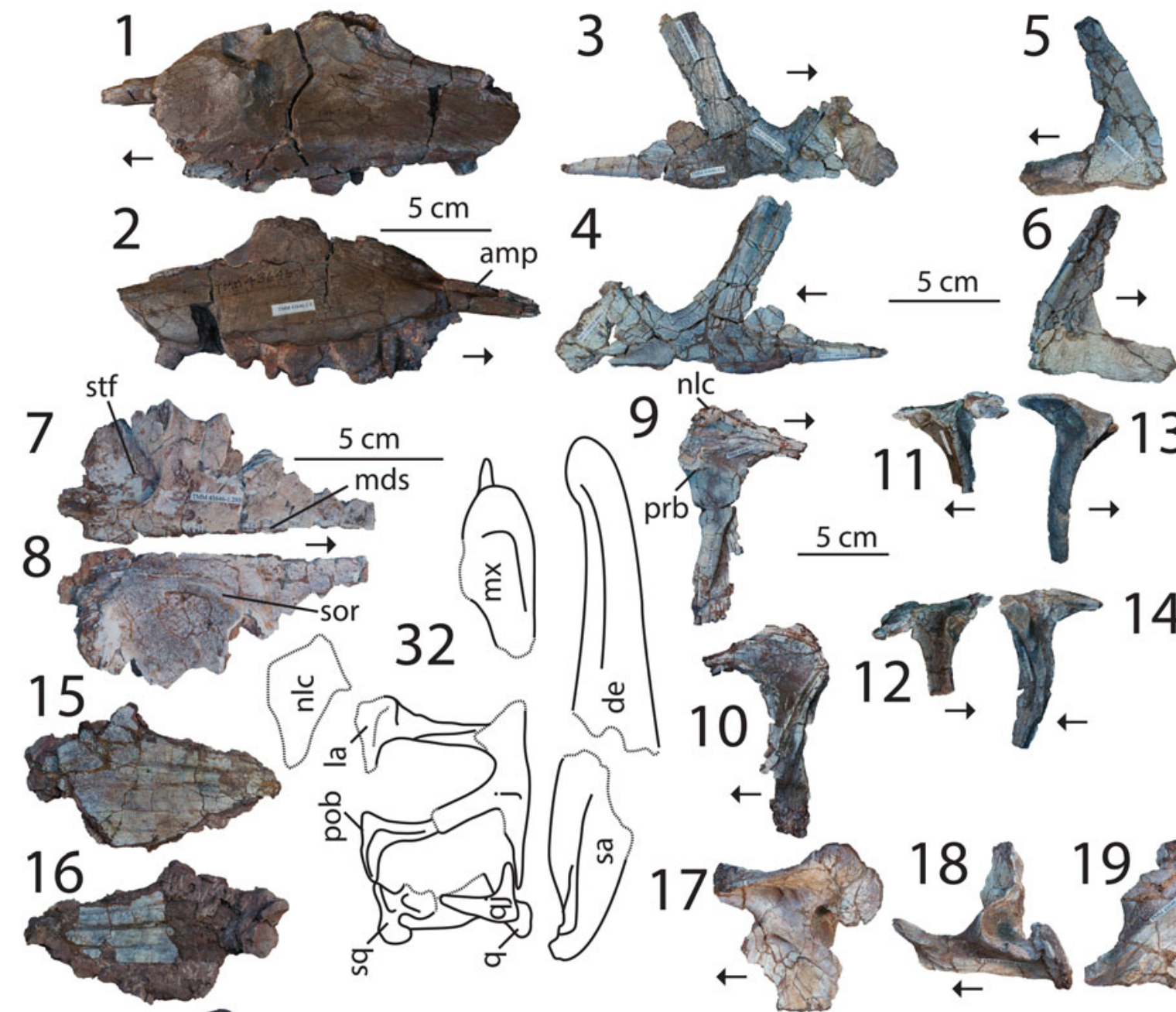

nlc
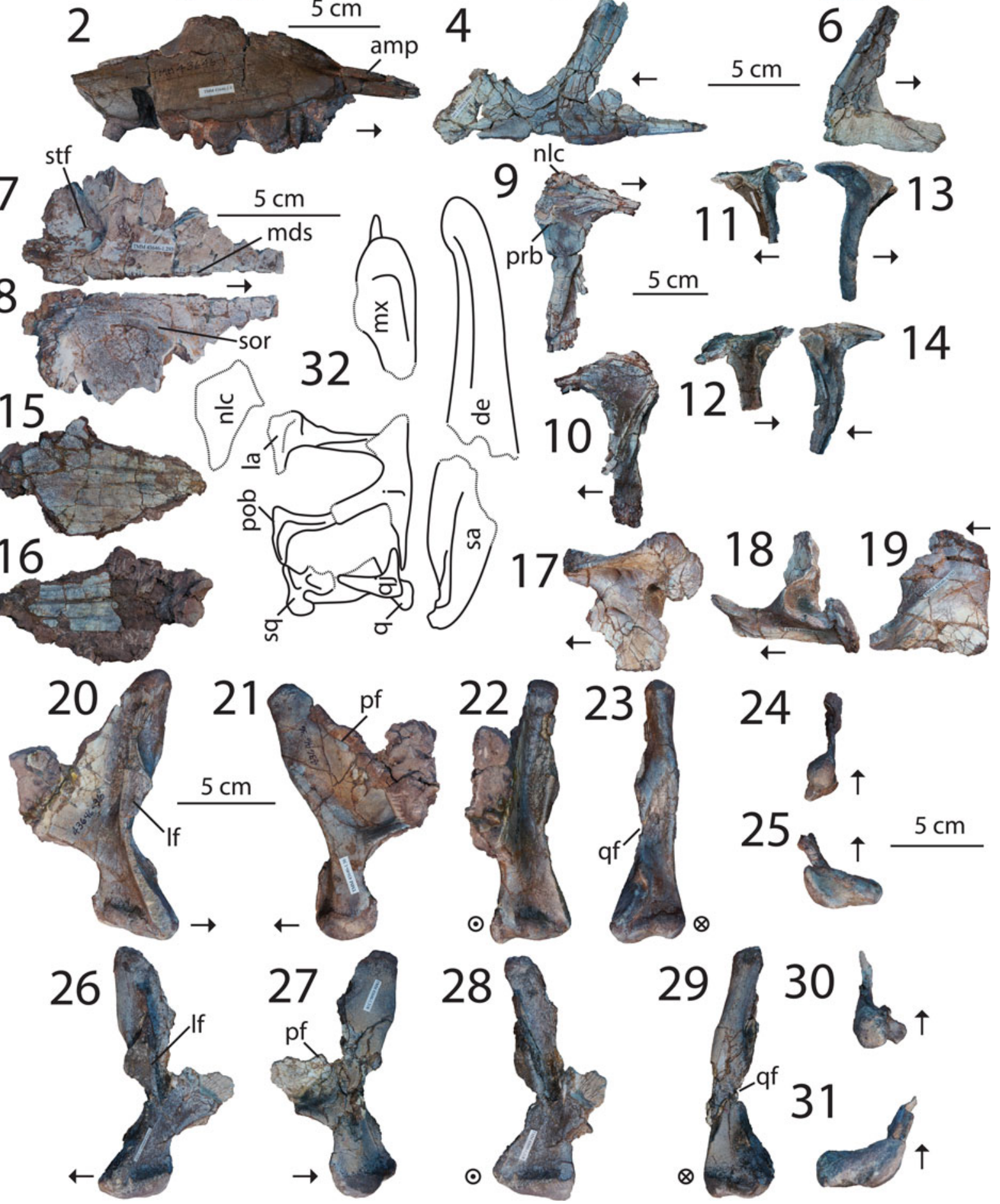

Figure 43. Dilophosaurus wetherilli referred specimen (TMM 43646-1): $(\mathbf{1 , 2})$ left maxilla, $(\mathbf{3}, \mathbf{4})$ right jugal, $(\mathbf{5}, \mathbf{6})$ left quadratojugal, $(\mathbf{7 , 8})$ left frontal, $(\mathbf{9 , 1 0})$ right lacrimal, $(\mathbf{1 1}, \mathbf{1 2})$ left postorbital, $(\mathbf{1 3}, \mathbf{1 4})$ right postorbital, $(\mathbf{1 5}, \mathbf{1 6})$ nasolacrimal crest, (17-19) left squamosal, $(\mathbf{2 0 - 2 5})$ left quadrate, (26-31) right quadrate, and (32) reconstructed skull elements; $(\mathbf{1}, \mathbf{3}, \mathbf{5}, 9, \mathbf{1 1}, \mathbf{1 3}, \mathbf{1 5}, \mathbf{1 7}, \mathbf{2 0}, \mathbf{2 6})$ lateral, $(\mathbf{2}, \mathbf{4}, \mathbf{6}, \mathbf{1 0}, \mathbf{1 2}, \mathbf{1 4}, \mathbf{1 6}, \mathbf{1 8}, \mathbf{2 1}, \mathbf{2 7}, 32)$ medial, $(\mathbf{7}, \mathbf{1 9}, \mathbf{2 4}, \mathbf{3 0})$ dorsal, $(\mathbf{8}, 18,25, \mathbf{3 1})$ ventral, $(\mathbf{2 2}$, $\mathbf{2 8}$ ) anterior, and $(\mathbf{2 3}, \mathbf{2 9})$ posterior view. Arrows point in anterior direction. $\mathrm{amp}=$ anteromedial process; de = dentary; $\mathrm{j}=\mathrm{jugal}$; la = lacrimal; lf $=$ lateral flange; $\mathrm{mds}$ $=$ midline suture $; \mathrm{mx}=$ maxilla; $\mathrm{nlc}=$ nasolacrimal crest $\mathrm{pf}=$ pterygoid flange $; \mathrm{pob}=$ postorbital $; \mathrm{prb}=$ preorbital boss; $\mathrm{q}=$ quadrate $; \mathrm{qf}=\mathrm{quadrate}$ foramen; $\mathrm{qj}=$ quadratojugal; $\mathrm{sa}=$ surangular; $\mathrm{sq}=$ squamosal; $\mathrm{sor}$ = supraorbital ridge; $\mathrm{stf}=$ supratemporal fossa. 


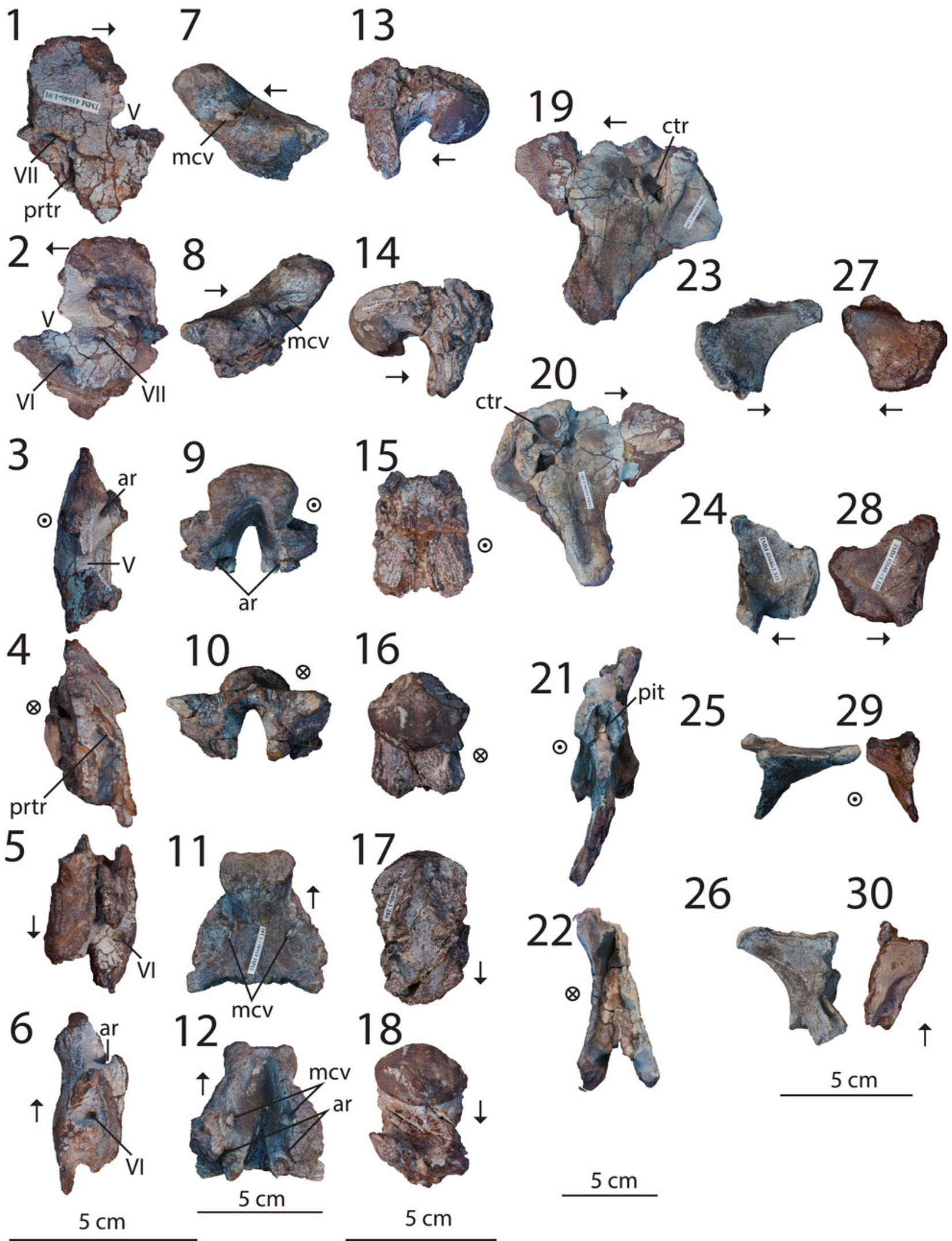

Figure 44. Dilophosaurus wetherilli referred specimen (TMM 43646-1): (1-6) right prootic, (7-12) supraoccipital, (13-18) basioccipital, (19-22) parabasisphenoid, (23-26) right laterosphenoid, and $(\mathbf{2 7 - 3 0})$ left laterosphenoid in $(\mathbf{1}, \mathbf{7}, \mathbf{8}, \mathbf{1 3}, \mathbf{1 4}, \mathbf{1 9}, \mathbf{2 0}, \mathbf{2 3}, \mathbf{2 7})$ lateral, $(\mathbf{2}, \mathbf{2 4}, \mathbf{2 8})$ medial, $(\mathbf{3}, \mathbf{9}, \mathbf{1 5}, \mathbf{2 1}, \mathbf{2 5}, \mathbf{2 9})$ anterior, $(\mathbf{4}, \mathbf{1 0}$, 16, 22) posterior, $(\mathbf{5 , 1 1}, \mathbf{1 7})$ dorsal, and $(\mathbf{6}, \mathbf{1 2}, \mathbf{1 8}, \mathbf{2 6}, \mathbf{3 0})$ ventral view. Arrows point in anterior direction. ar = auricular recess; ctr $=$ caudal tympanic recess; mcv = foramen for the middle cerebral vein; pit = pituitary fossa; prtr = prootic division of the rostral tympanic recess; $\mathrm{V}=$ opening for the trigeminal nerve; $\mathrm{VI}=$ foramen or canal for the abducens nerve; VII = foramen or canal for the facial nerve. 

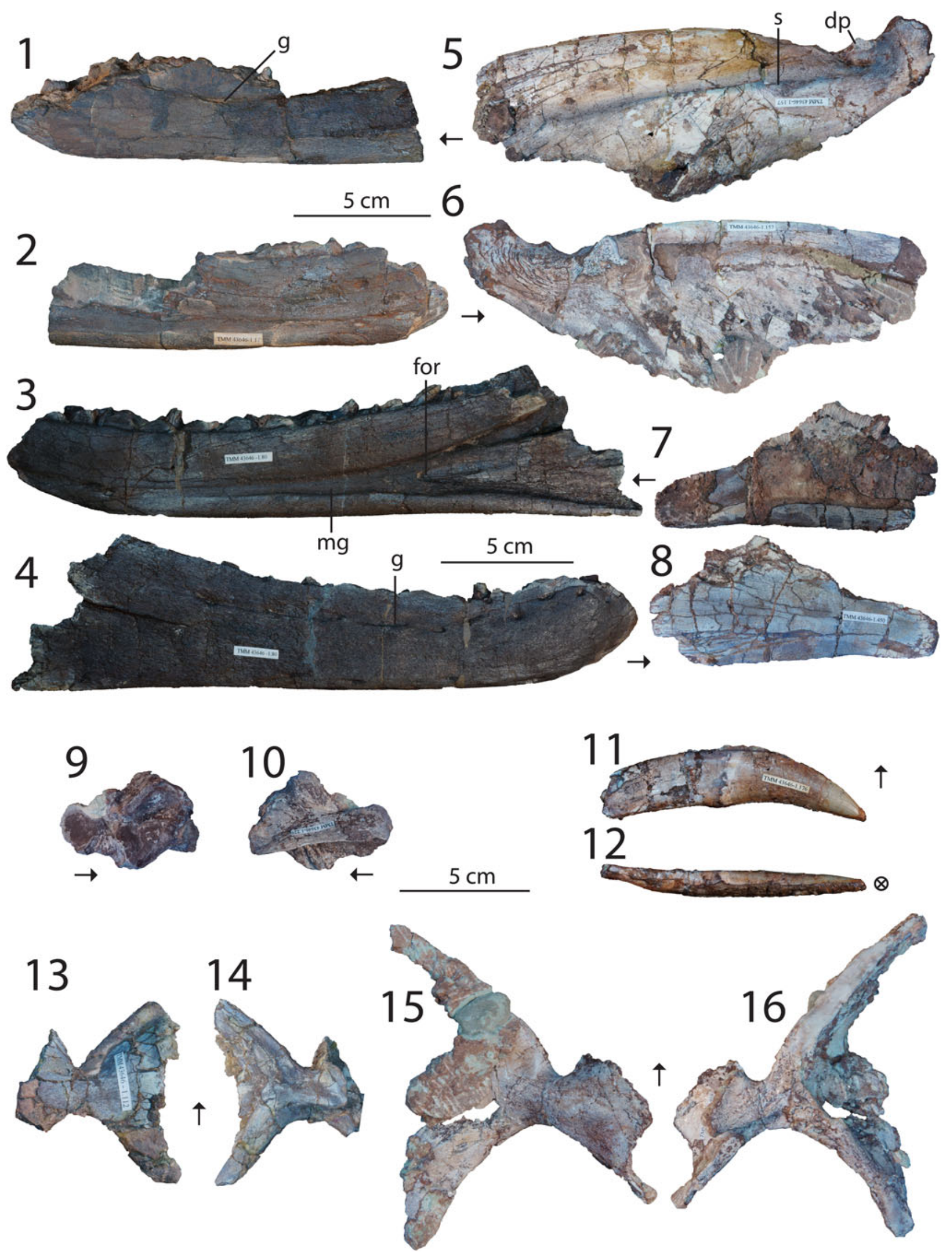

Figure 45. Dilophosaurus wetherilli referred specimen (TMM 43646-1): $(\mathbf{1}, \mathbf{2})$ left dentary, $(\mathbf{3}, \mathbf{4})$ right dentary, $(\mathbf{5}, \mathbf{6})$ left surangular, $(\mathbf{7}, \mathbf{8})$ right splenial, $(\mathbf{9}, \mathbf{1 0})$ right articular, $(\mathbf{1 1}, \mathbf{1 2})$ isolated tooth, $(\mathbf{1 3}, \mathbf{1 4})$ left pterygoid, and $(\mathbf{1 5}, \mathbf{1 6})$ right pterygoid in $(\mathbf{1}, \mathbf{4}, \mathbf{5}, \mathbf{7})$ lateral, $(\mathbf{2}, \mathbf{3}, \mathbf{6}, \mathbf{8}, \mathbf{1 1})$ medial, $(\mathbf{9}, \mathbf{1 3}, \mathbf{1 5})$ dorsal, $(\mathbf{1 0}, \mathbf{1 4}, \mathbf{1 6})$ ventral, (L) posterior, and (12) distal view. Arrows point in anterior direction. $\mathrm{dp}=$ dorsal process; for = foramen; $\mathrm{g}=\mathrm{groove} ; \mathrm{mg}=\mathrm{Meckelian}$ groove; $\mathrm{s}=\mathrm{shelf}$. 

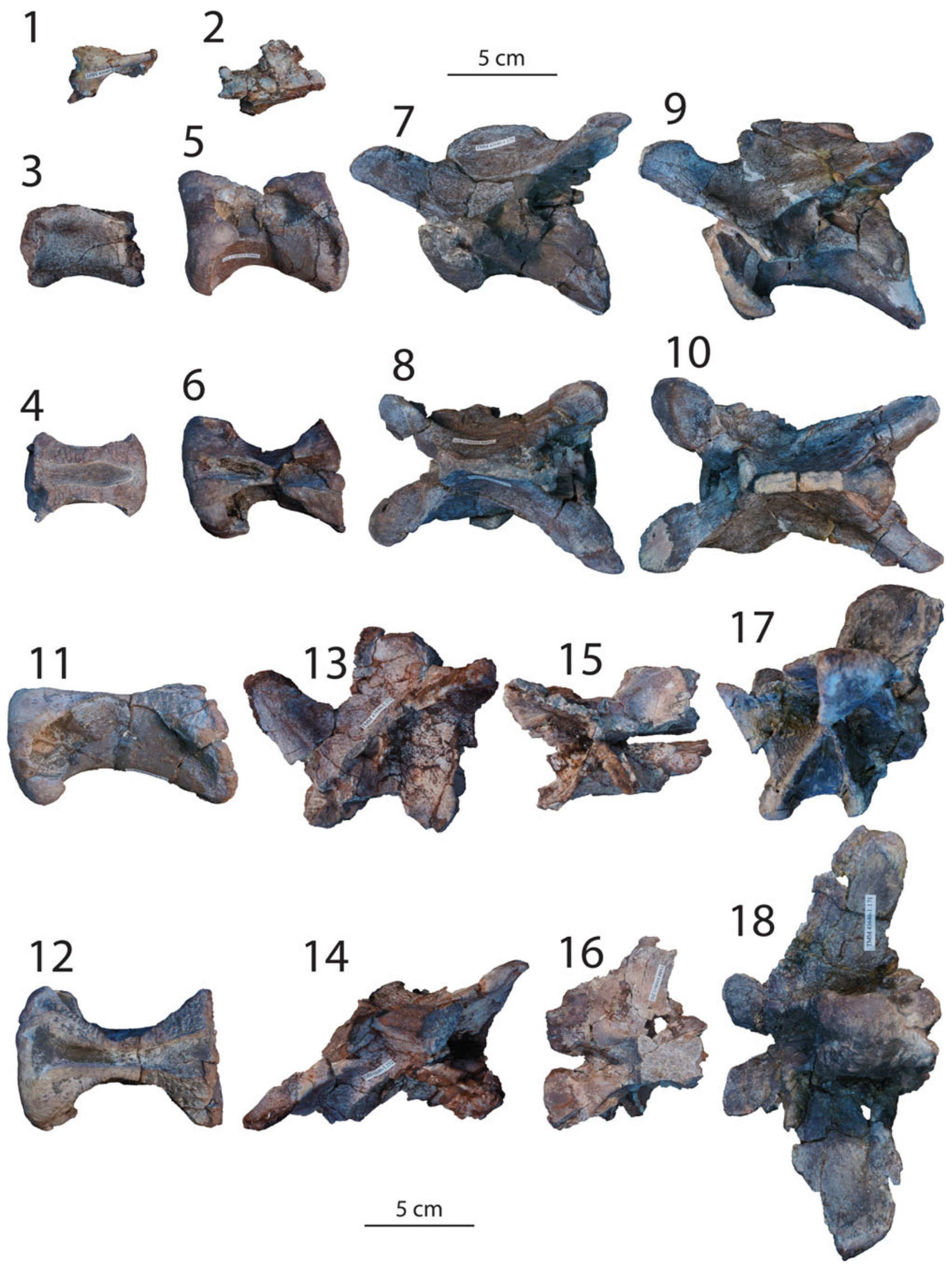

Figure 46. Dilophosaurus wetherilli referred specimen (TMM 43646-1): (1) right neurapophysis (reversed), (2) left cervical rib, $(\mathbf{3}, \mathbf{4})$ axis, and $(\mathbf{5}-\mathbf{1 8})$ seven cervical vertebrae in $(\mathbf{1}, \mathbf{2}, \mathbf{3}, \mathbf{5}, \mathbf{7}, \mathbf{9}, \mathbf{1 1}, \mathbf{1 3}, \mathbf{1 5}, \mathbf{1 7})$ left lateral and $(\mathbf{4}, \mathbf{6}, \mathbf{8}, \mathbf{1 0}, \mathbf{1 2}, \mathbf{1 4}, \mathbf{1 6}, \mathbf{1 8})$ dorsal view. Anterior is to the left. 


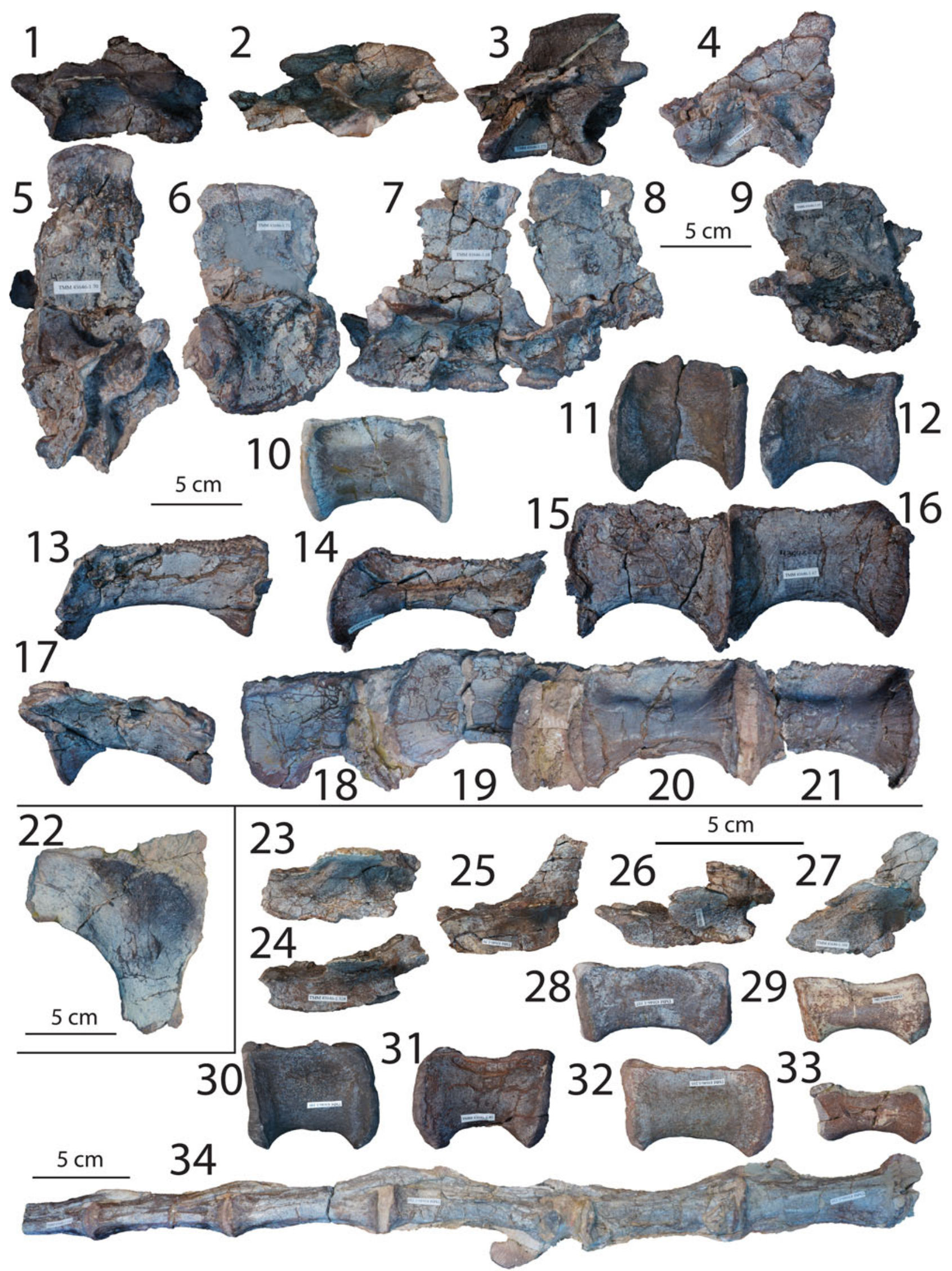

Figure 47. Dilophosaurus wetherilli referred specimen (TMM 43646-1): (1-21) trunk vertebrae, (22) caudosacral rib, and (23-34) caudal vertebrae in (1-21, 2333) left lateral, (34) right lateral, and (22) medial view. 

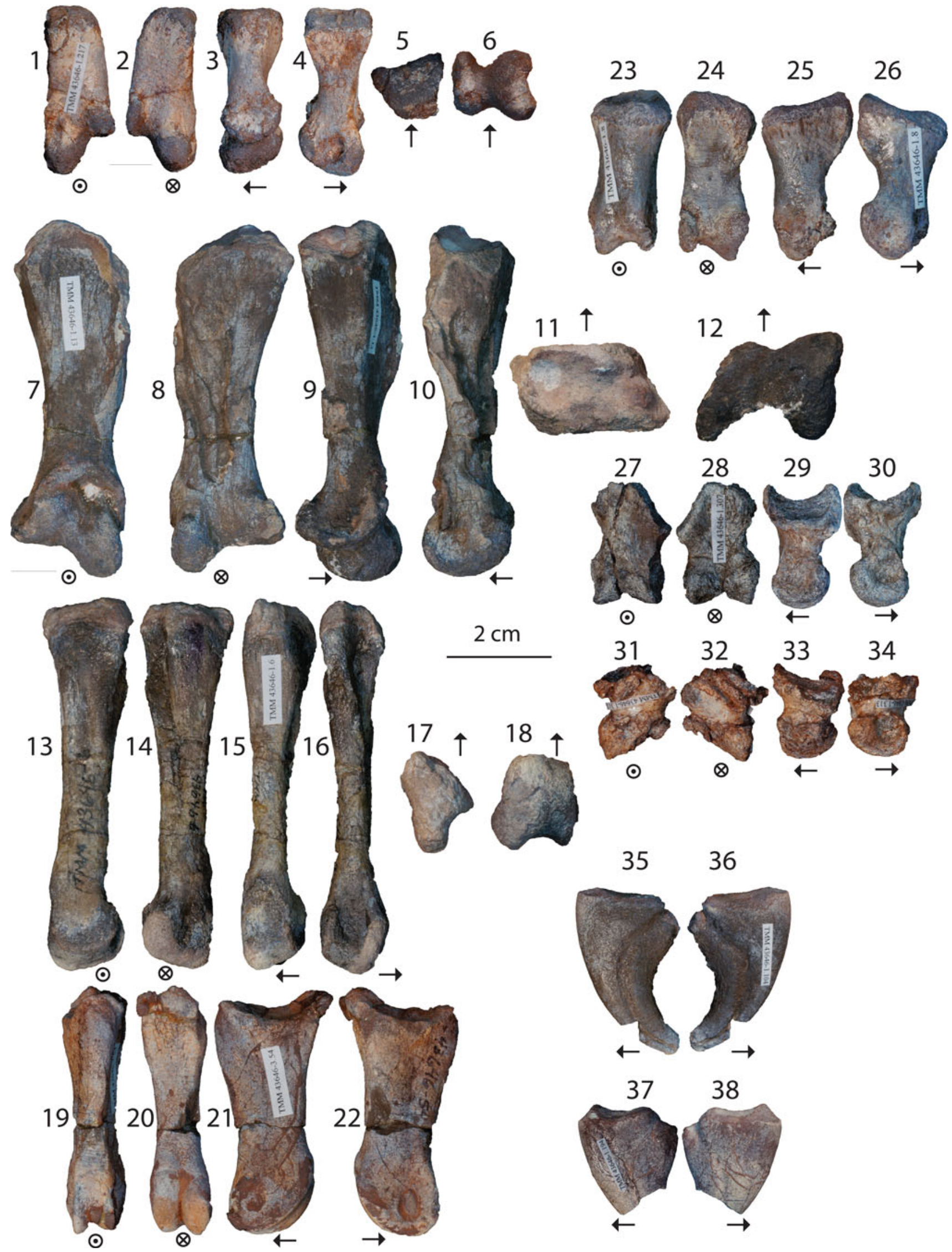

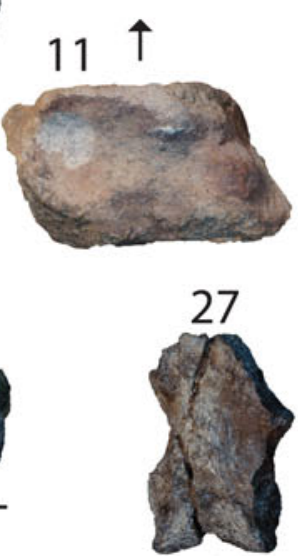

$\odot$
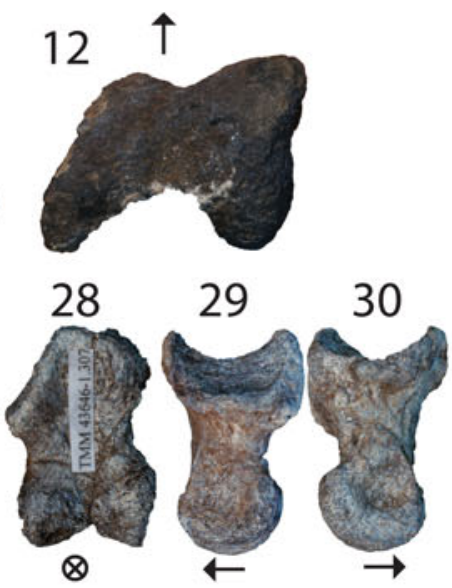

$2 \mathrm{~cm}$
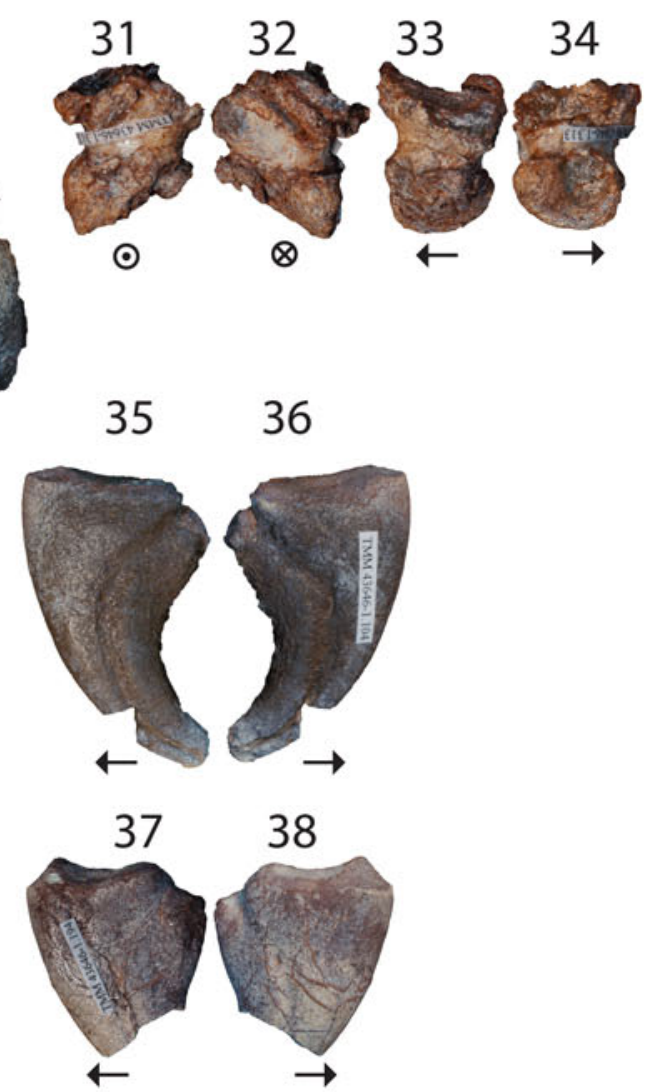

Figure 48. Dilophosaurus wetherilli referred specimen (TMM 43646-1): (1-6) right metacarpal I, (7-12) left metacarpal II, (13-18) right metacarpal III, and (1938) manual phalanges in $(1,7,13,19,23,27,31)$ dorsal, $(2,8,14,20,24,28,32)$ ventral, $(3,9,15,21,25,29,33,35,37)$ lateral, $(4,10,16,22,26,30,34,36,38)$ medial, proximal $(\mathbf{5}, \mathbf{1 1}, \mathbf{1 7})$, and $(\mathbf{6}, \mathbf{1 2}, \mathbf{1 8})$ distal view. Arrows point in dorsal direction. 


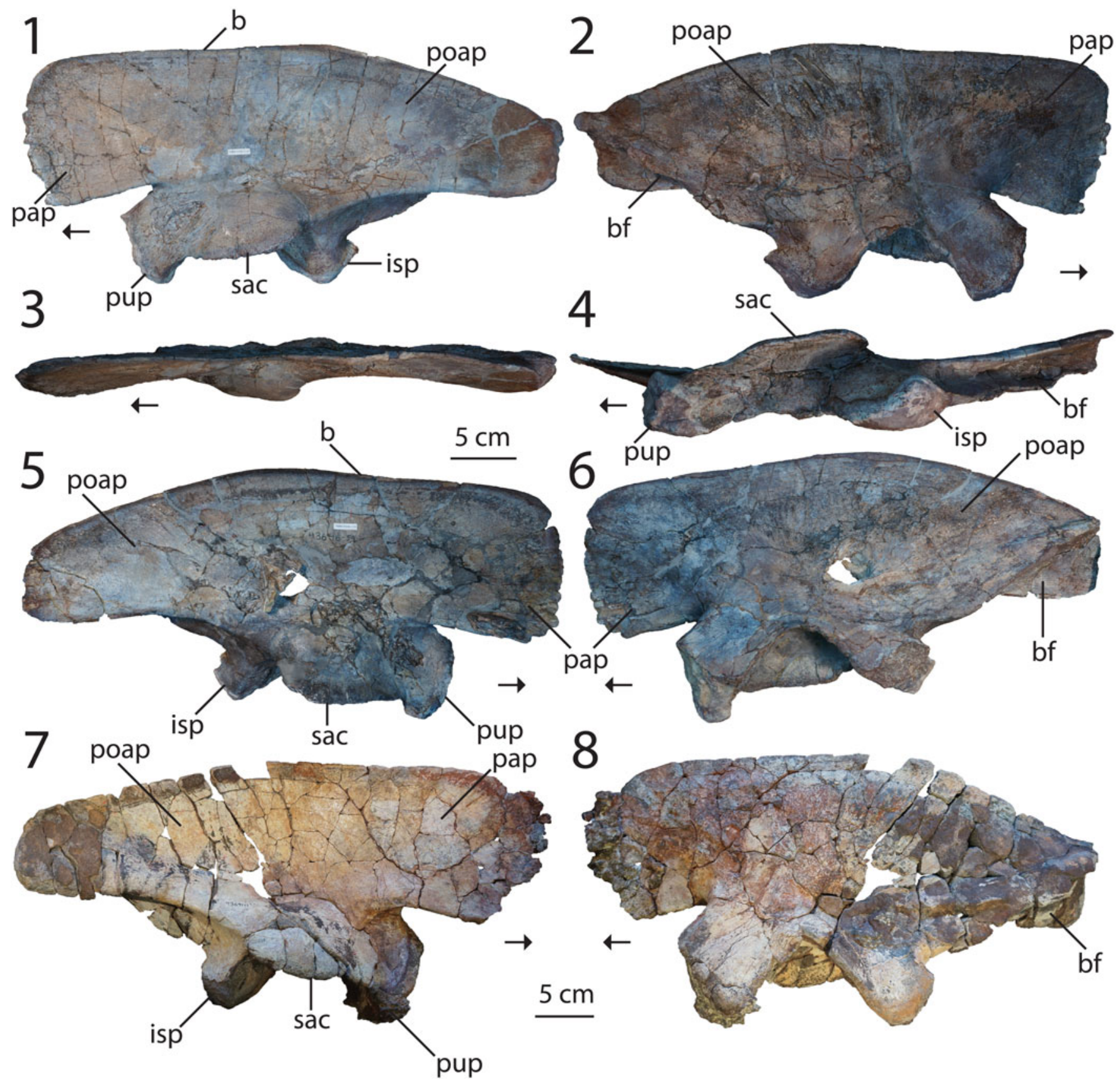

Figure 49. Dilophosaurus wetherilli referred specimen (TMM 43646-1): (1-4) left ilium and (5, 6) right ilium; Dilophosaurus wetherilli referred specimen (TMM 43691-1): $(\mathbf{7}, \mathbf{8})$ right ilium. $(\mathbf{1}, \mathbf{5}, \mathbf{7})$ Lateral, $(\mathbf{2}, \mathbf{6}, \mathbf{8})$ medial, $(\mathbf{3})$ dorsal, and $(\mathbf{4})$ ventral view. Arrows point in anterior direction. $\mathrm{b}=\mathrm{blade} ; \mathrm{bf}=\mathrm{brevis}$ fossa; isp $=$ ischial peduncle; pap = preacetabular process; poap = postacetabular process; pup = pubic peduncle; sac $=$ supraacetabular crest.

concave medially in posterior view, but the proximal and distal ends are concave laterally. The fourth trochanter is a tall ridge. Its middle region has parallel striations on the medial and lateral sides that are perpendicular to the long access of the femur. The most proximal extent of the fourth trochanter is around the same proximodistal level as the posterolateral tuber and the distal end of the anterior trochanter. A distal rugosity (=distal tuberosity; Brochu, 2003) lies near the center of the anterior surface of the ventral half of the femur (Figs. 20.2, 20.8, 42.2, 42.6, 52.4, 52.6), similar to that of Dromomeron romeri Irmis et al., 2007 and D. gregorii Nesbitt et al., 2009a (Nesbitt et al., 2009a; Nesbitt and Ezcurra, 2015) and Eodromaeus murphi (Martínez et al., 2011). This area is not raised in Dilophosaurus wetherilli, but it is covered in a rough patch of bone consistent with the texture of a region of muscle insertion, such as the M. femorotibialis externus (Hutchinson, 2001; Carrano and Hutchinson, 2002).

A shallow triangular depression below the distal rugosity separates the anterior margins of the distal medial and lateral condyles of the femur, which extends distally and divides them in distal view, as well. The entepicondyle and ectepicondyles of the femur are not pronounced or set apart from the sides of the distal condyles, although the lateral condyle is 

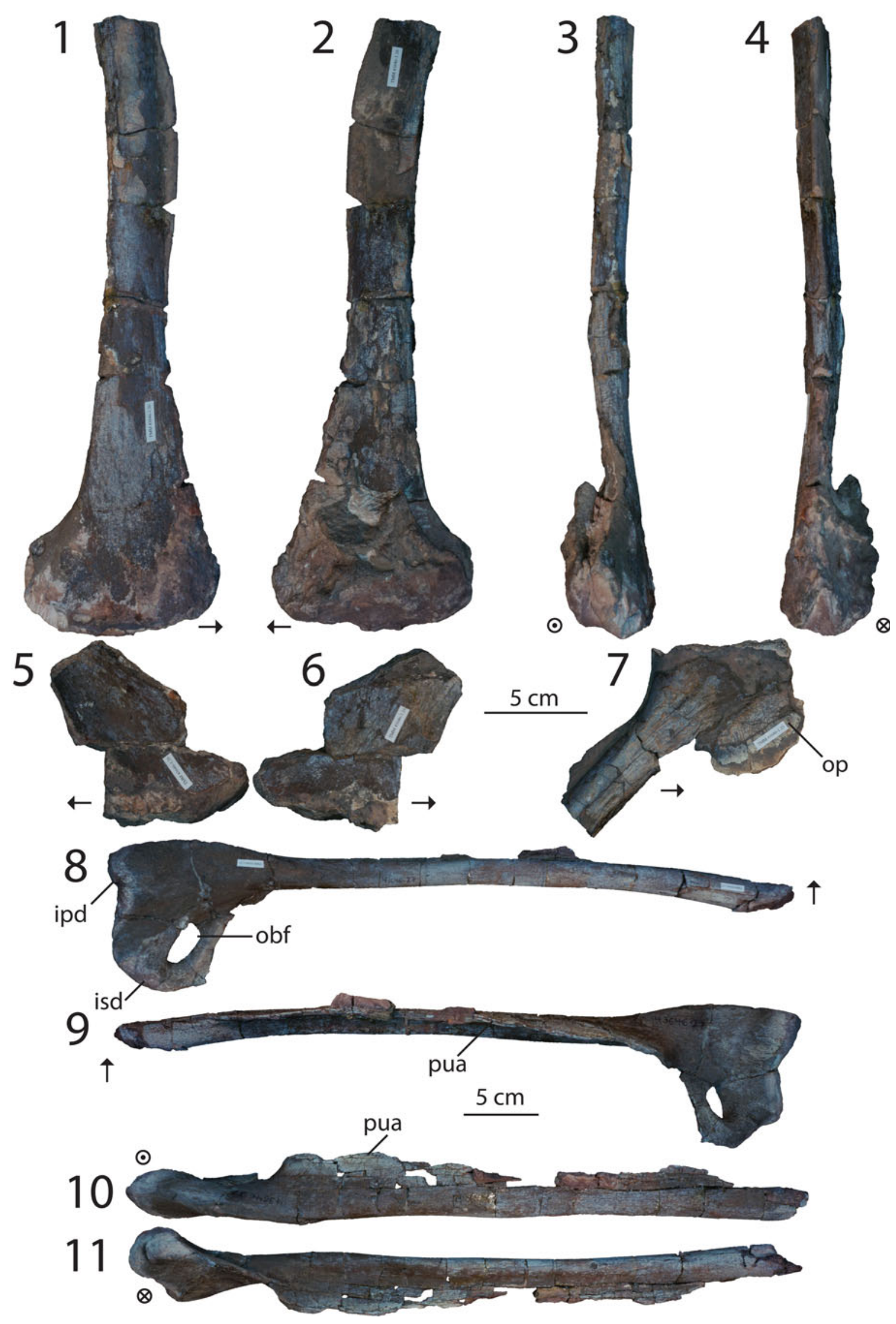

Figure 50. Dilophosaurus wetherilli referred specimen (TMM 43646-1): (1-4) distal end of right ischium, (5, 6) distal end of left ischium, (7) proximal end of right ischium, and $(\mathbf{8}-\mathbf{1 1})$ right pubis in $(\mathbf{1}, \mathbf{5}, \mathbf{7}, \mathbf{8})$ lateral, $(\mathbf{2}, \mathbf{6}, \mathbf{9})$ medial, $(\mathbf{3}, \mathbf{1 0})$ anterior, and $(\mathbf{4 , 1 1})$ posterior view. Arrows point in anterior direction. ipd = iliac pedicle; isd = ischial pedicle; obf = obturator foramen; op = obturator process; pua = pubic apron. 


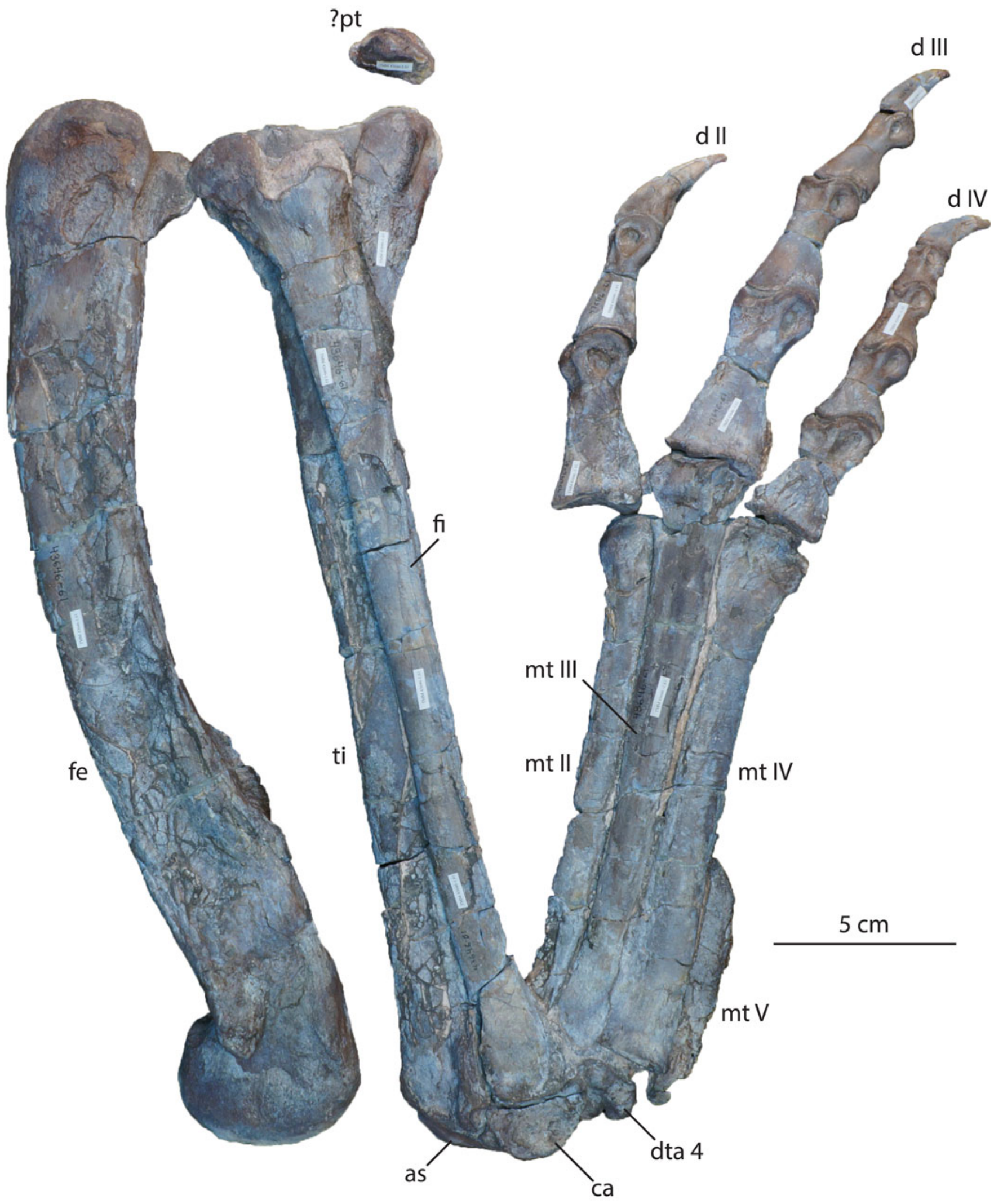

Figure 51. Dilophosaurus wetherilli referred specimen (TMM 43646-1): articulated right hindlimb, including femur, tibia, fibula, tarsus, pes, and possible patella, in anterolateral view. as = astragalus; $\mathrm{ca}=$ calcaneum; $\mathrm{d}=$ digit; $\mathrm{dta}=$ distal tarsal; $\mathrm{fe}=$ femur; $\mathrm{fi}=$ fibula; $\mathrm{mt}=$ metatarsal; $\mathrm{pt}=\mathrm{patella}$; $\mathrm{t}=\mathrm{tibia}$. 

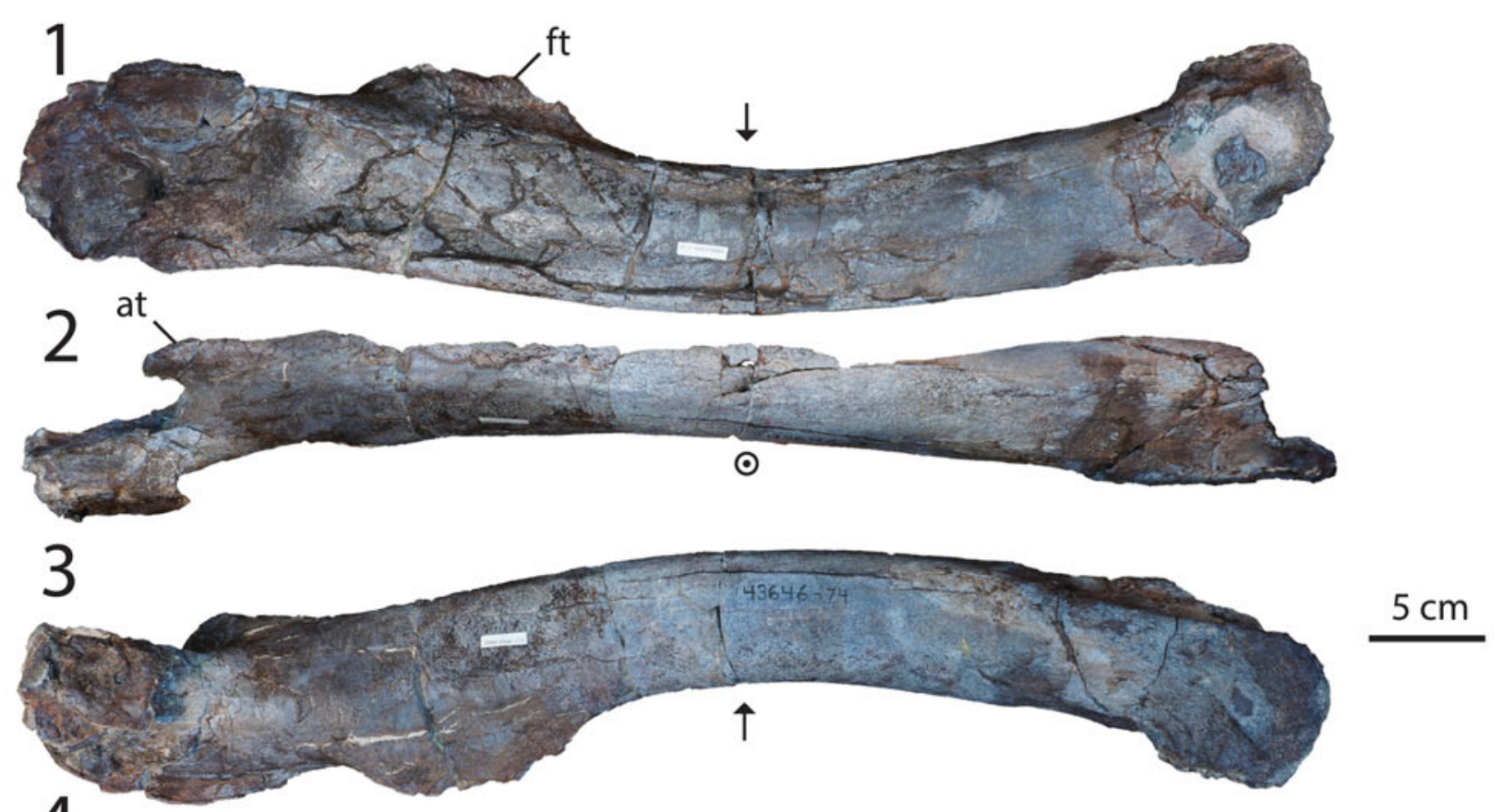

4
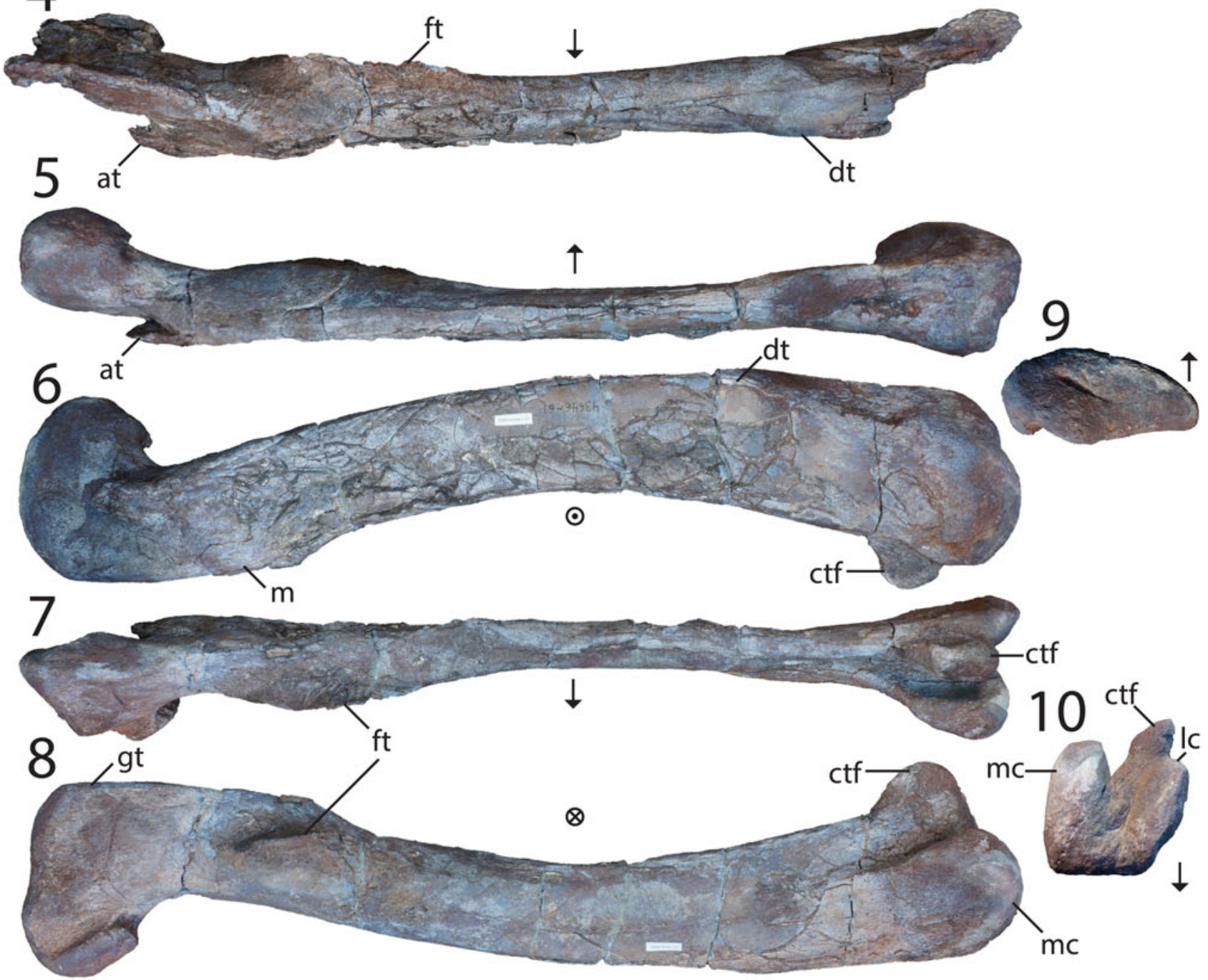

Figure 52. Dilophosaurus wetherilli referred specimen (TMM 43646-1): (1-4) left femur and (5-10) right femur in (1, 5) lateral, (2, 6) anterolateral, $(\mathbf{3 , 7})$ medial, (4) posterolateral, (8) posteromedial, (9) proximal, and (10) distal view. Arrows point in anterior direction. at $=$ anterior trochanter; $\mathrm{ctf}=\mathrm{crista}$ tibiofibularis; $\mathrm{dt}=\mathrm{distal}$ tuberosity; $\mathrm{ft}=$ fourth trochanter; $\mathrm{gt}=$ greater trochanter; $\mathrm{lc}=$ lateral condyle; $\mathrm{m}=$ mound; $\mathrm{mc}=$ medial condyle. 

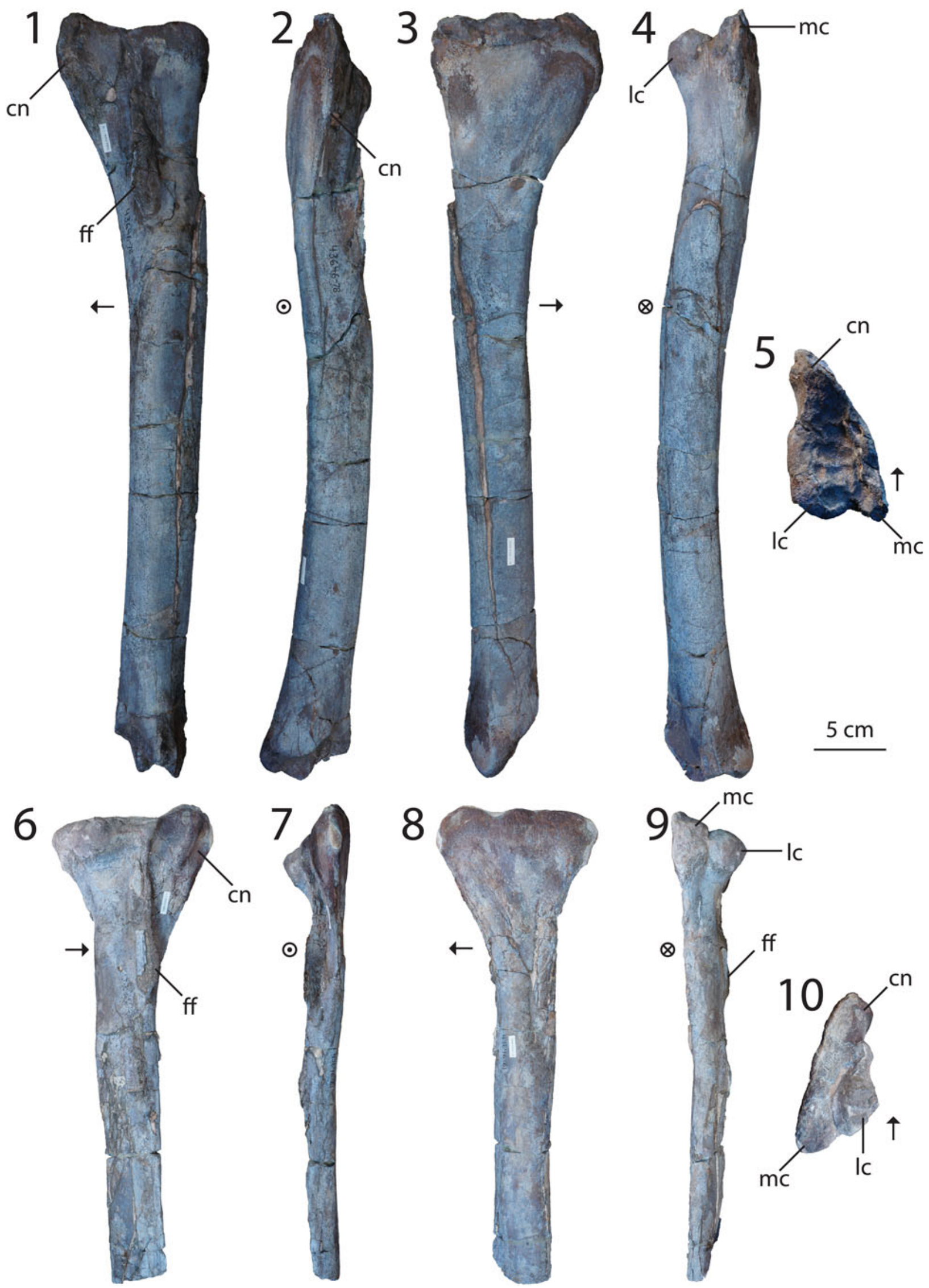

Figure 53. Dilophosaurus wetherilli referred specimen (TMM 43646-1): (1-5) left tibia and (6-10) proximal end of right tibia in $(\mathbf{1 , 6})$ lateral, $(\mathbf{2}, \mathbf{7})$ anterior, $(\mathbf{3}, \mathbf{8})$ medial, $(\mathbf{4 , 9})$ posterior, and $(\mathbf{5 , 1 0})$ proximal view. Arrows point in anterior direction. $\mathrm{cn}=$ cnemial crest; $\mathrm{ff}=$ fibular flange; $\mathrm{lc}=$ lateral condyle; $\mathrm{mc}=\mathrm{medial}$ condyle. 

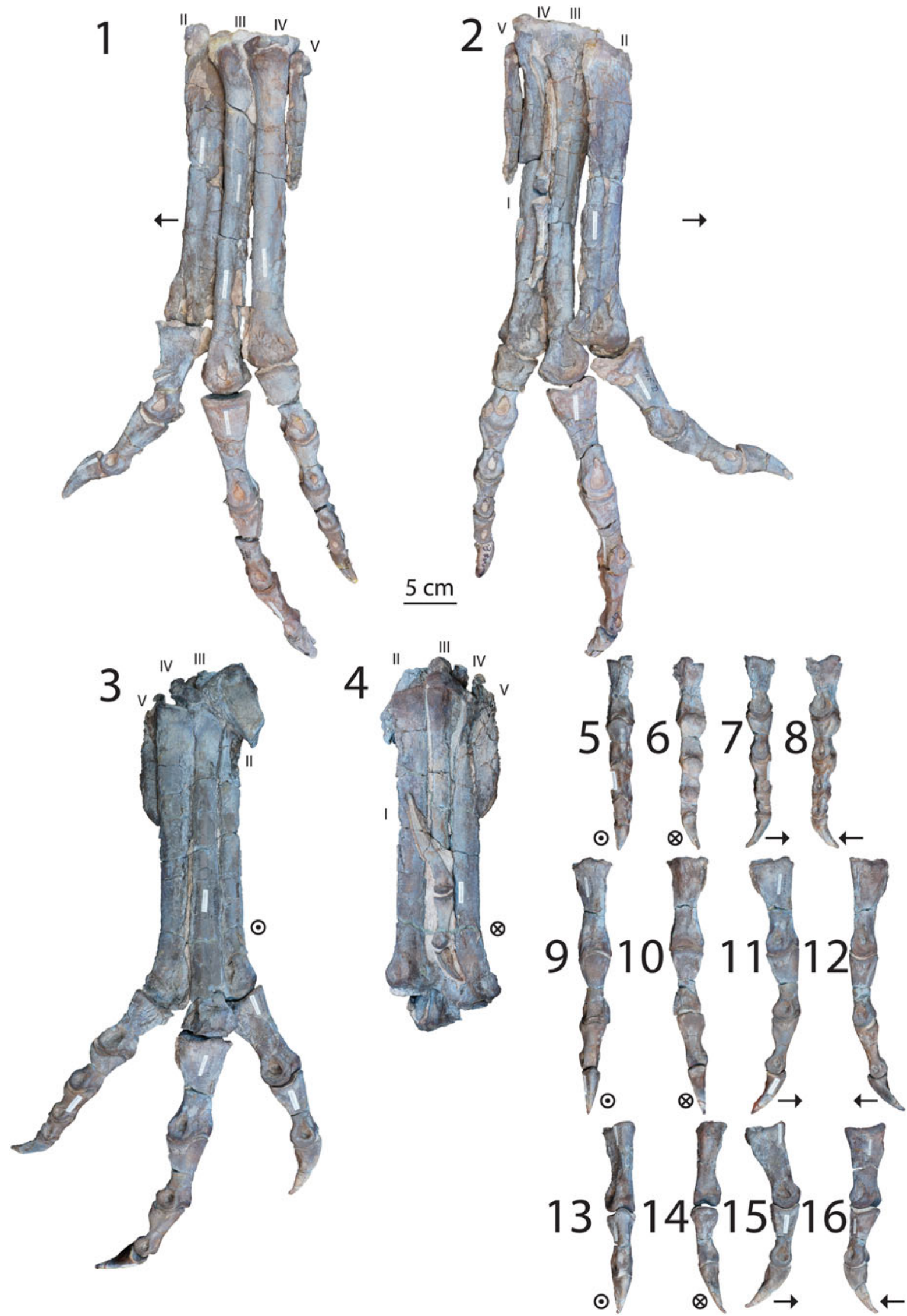

Figure 54. Dilophosaurus wetherilli referred specimen (TMM 43646-1): $(\mathbf{1}, \mathbf{2})$ articulated left pes, $(\mathbf{3}, \mathbf{4})$ articulated right pes, and articulated $(\mathbf{5 - 8})$ right pedal digit IV, (9-12) right pedal digit III, and (13-16) right pedal digit II in $(\mathbf{1 , 3})$ dorsolateral, $(\mathbf{2}, \mathbf{4})$ ventromedial, $(\mathbf{5 , 9}, \mathbf{1 3})$ dorsal, $(\mathbf{6}, \mathbf{1 0}, \mathbf{1 4})$ ventral, $(\mathbf{7}, \mathbf{1 1}, \mathbf{1 5})$ lateral, and $(\mathbf{8}$, 12, 16) medial view. Arrows point in dorsal direction. 


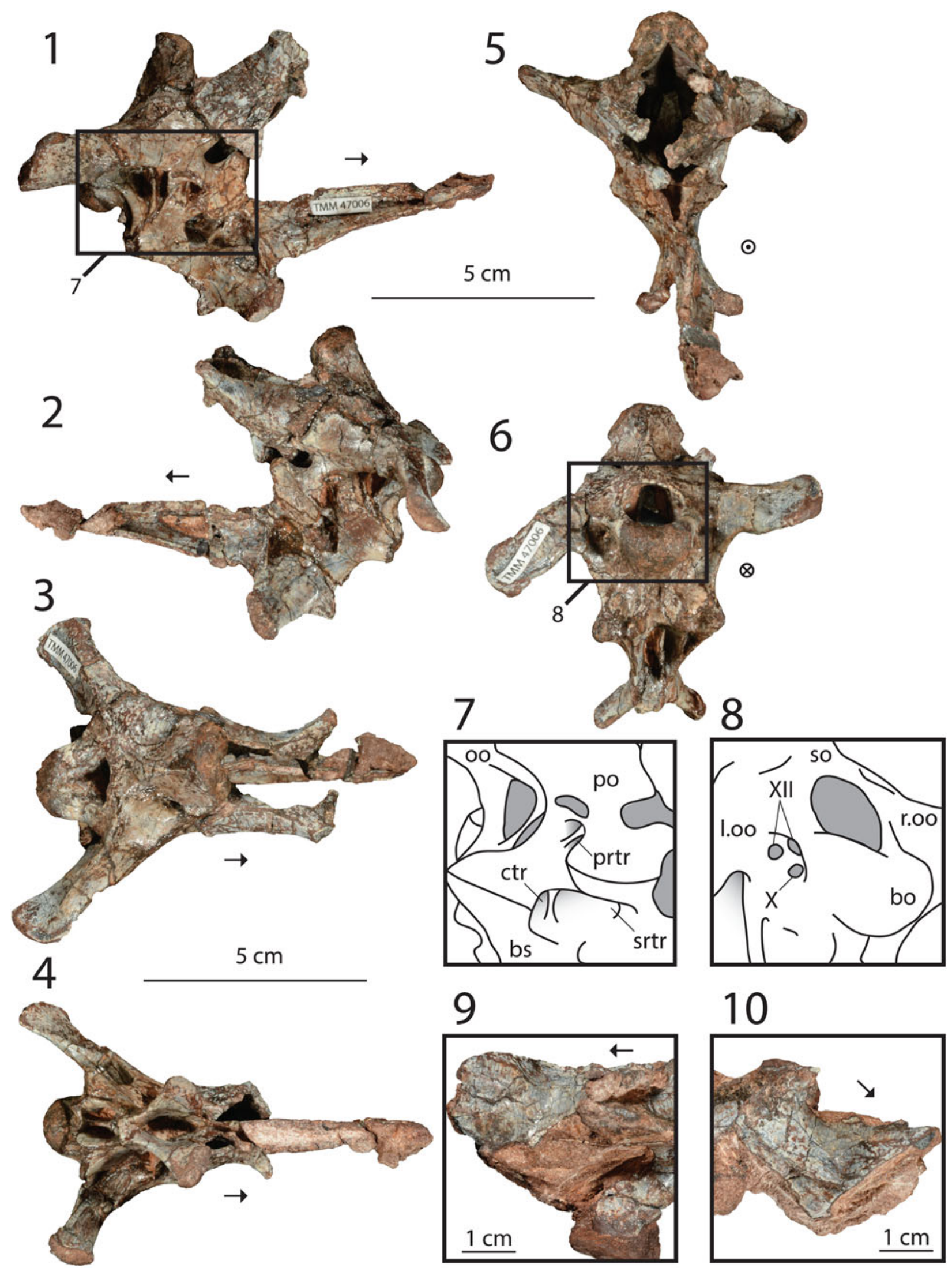

Figure 55. Dilophosaurus wetherilli referred specimen (TMM 47006-1): (1-8) articulated braincase and left parietal $(\mathbf{9}, \mathbf{1 0})$ in $(\mathbf{1})$ right lateral, $(\mathbf{2}, \mathbf{9})$ left lateral, $(\mathbf{3}$, 10) dorsal, (4) ventral, (5) anterior, (6) posterior, and $(\mathbf{7}, \mathbf{8})$ oblique views. Arrows point in anterior direction. bo = basioccipital; bs = basisphenoid; ctr = caudal tympanic recess; $1.00=$ left otooccipital; oo = otooccipital; $\mathrm{po}=$ postorbital; prtr $=$ prootic division of the rostral tympanic recess; r.oo $=$ right otooccipital; so $=$ supraoccipital; srtr = subotic division of the rostral tympanic recess; $\mathrm{X}=$ foramen or canal for the vagus nerve; $\mathrm{XII}=$ foramen or canal for the hypoglossal nerve. 
more expanded mediolaterally because the medial surface of the medial distal condyle is flat and curved anteroventrally, a condition that is typical in many Triassic and Jurassic theropods such as Coelophysis bauri (Colbert, 1989), Syntarsus kayentakatae (Rowe, 1989; Tykoski, 1998), and Allosaurus fragilis (Madsen, 1976). This flattened medial surface forms a right angle with the anteromedial margin of the femur in distal view. The distal end of the femur is divided into lateral and medial condyles as well as the crista tibiofibularis (Figs. 20.6, 20.9, 52.10). The lateral condyle and crista tibiofibularis are set apart posteriorly from the medial condyle by a deep groove; the medial condyle and combined lateral condyle and crista tibiofibularis project backward significantly from the shaft of the femur (Figs. 20.6, 20.9, $52.10,61.9)$. The medial condyle and crista tibiofibularis are subelliptical in distal outline. The distal outline of the lateral condyle is more rectangular and mediolaterally wider than that of the medial condyle. An oblique groove divides the crista tibiofibularis from the lateral condyle; it starts along the lateral surface of the crista tibiofibularis, extends down and around the lateral condyle, and terminates where it reaches the anterolateral corner of the medial condyle in distal view. This is unlike Zupaysaurus rougieri (Ezcurra and Novas, 2007), in which the two structures are separated by a broad concavity. The femur of Dilophosaurus wetherilli does not have an infrapopliteal ridge between the medial condyle and crista tibiofibularis in posterior view, unlike Ceratosaurus nasicornis (Madsen and Welles, 2000), coelophysoids (Raath, 1977; Colbert, 1989; Rowe, 1989), and the tetanuran Sinraptor dongi (Currie and Zhao, 1993).

?Patella.-A rounded, subtriangular bone was found in the knee region of the articulated right hindlimb of TMM 43646-1 (Fig. 51). It is flat on one side and has a subcircular, rounded tuber on the other side. If this is indeed a sesamoid homologous to the patella of birds, it may be the earliest patella preserved in the stem-avian lineage (Barnett and Lewis, 1958; Regnault et al., 2014). However, given the independent acquisition of a dorsal sesamoid in the knee (as opposed to the ventral sesamoid more associated with the cnemial crest of the tibia; Barnett and Lewis, 1958) within Tetrapoda and its absence in other nonavialan neotheropods (Haines, 1942; Regnault et al., 2014, 2016; Abdala et al., 2017; Samuels et al., 2017), this bone may be present homoplastically in Dilophosaurus wetherilli.

Tibia.-The tibia is subequal to the femur in length. The proximal surface is concave similar to that of most neotheropods (Nesbitt and Ezcurra, 2015; Figs. 21.5, 21.11, 33.7, 53.5, 53.10, $61.10,61.12)$. The proximal medial and lateral condyles of the tibia are distinguished from one another posteriorly by a $\mathrm{V}$-shaped groove. The proximal lateral and medial condyles are subequal in size and shape. The proximal surface of the tibia slopes gently downwards towards the cnemial crest. The medial side of the cnemial crest is confluent with the medial side of the proximal medial condyle, but the lateral condyle and cnemial crest are separated laterally by a broad concavity. The anterior margin of the cnemial crest is squared off in proximal view. The cnemial crest is subtriangular and short in lateral view (Figs. 21.1, 21.7, 33.3, 53.1, 53.6), and it recedes gently back onto the anterior surface of the tibial shaft below the proximal condyles.

A fibular flange lies on the lateral surface of the proximal end of the tibia below the distal termination of the cnemial crest (Figs. 21.1, 21.6, 33.3, 53.1, 53.6). This flange is long, tall, and straight, and articulates with a corresponding depression on the medial surface of the fibula. The fibular flange is separated from the proximal surface of the tibia in Dilophosaurus wetherilli and Tachiraptor admirabilis (Langer et al., 2014), unlike that of some other early theropods in which it extends nearly from the proximal articular surface of the tibia (von Huene, 1934; Raath, 1977; Colbert, 1989; Rowe, 1989; Carrano et al., 2005; Martínez et al., 2011; Nesbitt and Ezcurra, 2015). Herrerasaurids, Tawa hallae, and Chindesaurus bryansmalli lack the fibular flange all together (Novas, 1994; Long and Murry, 1995; Bittencourt and Kellner, 2009; Nesbitt et al., 2009b).

The shaft of the tibia is subcircular in cross section and tapers distally towards the articular surface with the astragalus. The distal end of the tibia is expanded mediolaterally in its articulation with the tarsus. The distal end of the tibia is subrectangular in Dilophosaurus wetherilli and most non-averostran dinosauromorphs (Fig. 33.8), but the distal outline of the tibia is subsquare in herrerasaurids (Novas, 1994; Bittencourt and Kellner, 2009) and subtriangular in averostrans (Madsen, 1976; Bonaparte, 1986; Madsen and Welles, 2000; Brochu, 2003). A longitudinal ridge is present on the lateral surface of the distal end of the tibia like that found in Camposaurus arizonensis Hunt et al., 1998 (Ezcurra and Brusatte, 2011) and Ceratosaurus nasicornis (Madsen and Welles, 2000). The medial side of the distal end of the tibia is delineated by a short ridge that is subparallel to the bottom edge of the anterolateral process. This ridge is found in the large coelophysoid UCMP 129618/ PEFO 21373 (Padian, 1986) and is next to the groove found on Camposaurus arizonensis (Ezcurra and Brusatte, 2011).

Both the anterolateral process (=astragalar overhang) and posterolateral process (=postfibular plate of Welles, 1984) are present in Dilophosaurus wetherilli and delineate the anterior triangular articular surface for the ascending process of the astragalus (Figs. 21.6, 21.12, 33.8). The anterolateral process forms an oblique, thin platform for the reception of the anterior process of the astragalus. The posterolateral process of the tibia extends distally and laterally, tapering to a point laterally in distal view similar to the posterolateral process of the tibiae of Syntarsus rhodesiensis (Raath, 1977), Cryolophosaurus ellioti (Smith et al., 2007), and early averostrans (Madsen, 1976; Madsen and Welles, 2000; Nesbitt and Ezcurra, 2015). A deep notch is present on the posteromedial corner of the distal end of the tibia that covers the raised process on the posteromedial corner of the astragalus. This notch is also deep in Zupaysaurus rougieri (Ezcurra and Novas, 2007), Cryolophosaurus ellioti (Smith et al., 2007), and early averostrans (Madsen, 1976; Madsen and Welles, 2000; Nesbitt and Ezcurra, 2015), it is shallow in Eodromaeus murphi (Martínez et al., 2011), Lepidus praecisio (Nesbitt and Ezcurra, 2015), and coelophysids (Colbert, 1989; Ezcurra and Brusatte, 2011), and it is absent in herrerasaurids (Novas, 1994; Bittencourt and Kellner, 2009), Tawa hallae (Nesbitt et al., 2009b), and Chindesaurus bryansmalli (Long and Murry, 1995).

Fibula.-The fibula is long and slender (Fig. 22). It articulates closely with the lateral side of the tibia and extends down to contact the calcaneum (Figs. 33.1, 33.2, 51). The fibula is concave medially in proximal view; the anterior end is round and 

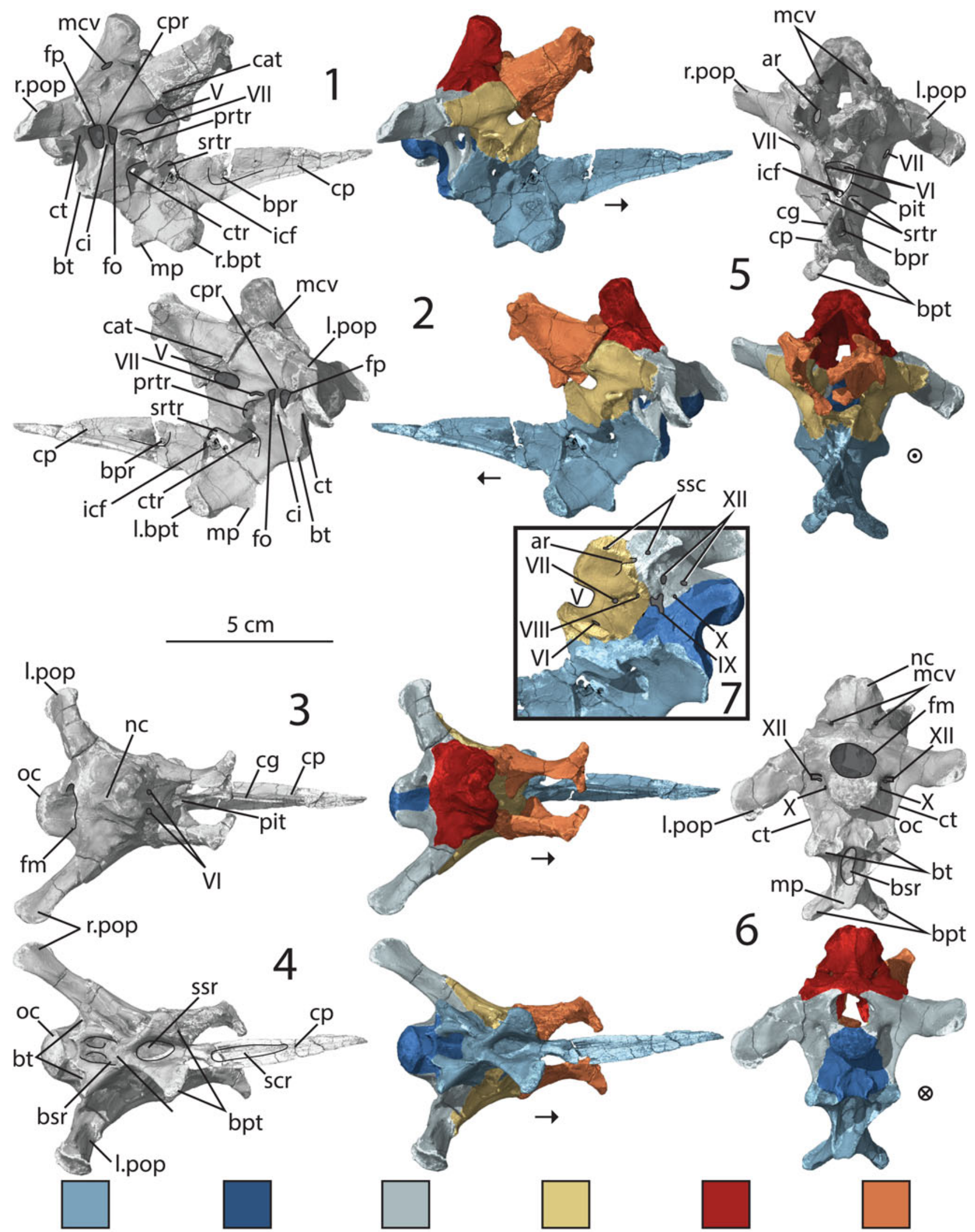

basisphenoid basioccipital otooccipital

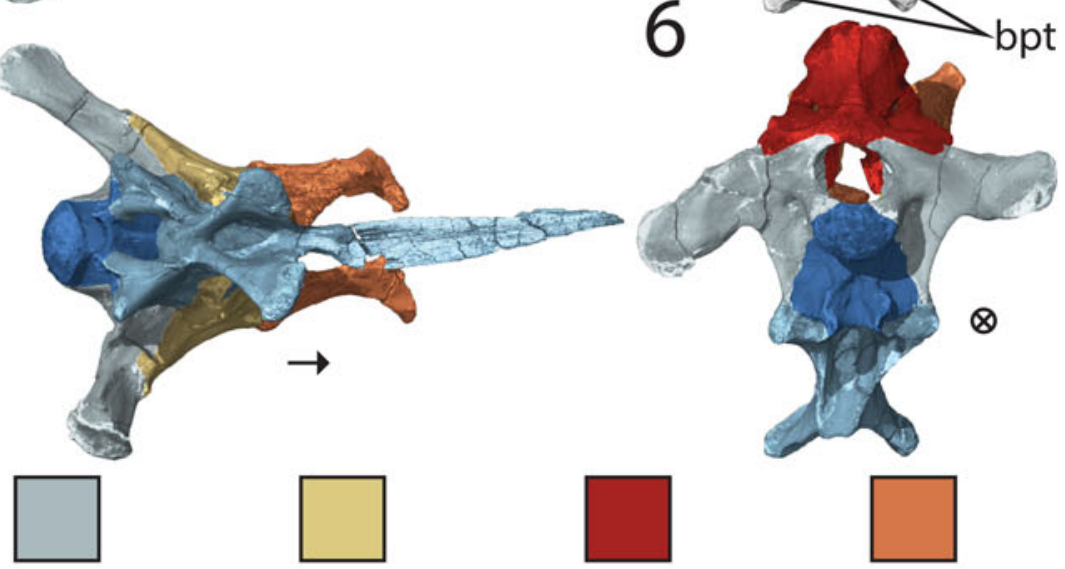

prootic supraoccipital laterosphenoid 
Figure 56. Dilophosaurus wetherilli referred specimen (TMM 47006-1): (1-6) articulated braincase (CT volume renderings with matrix digitally removed) in (1) right lateral, $(\mathbf{2}, \mathbf{7})$ left lateral, (3) dorsal, (4) ventral, $(\mathbf{5})$ anterior, and (6) posterior view. The left side of (7) has been removed to show the medial surface of right prootic and otooccipital. Arrows point in anterior direction. ar $=$ auricular recess; $b p r=$ basipterygoid recess; $b p t=$ basipterygoid process; $b s r=$ basisphenoid recess; $\mathrm{bt}=$ basal tuber; $\mathrm{cat}=$ crista antotica $; \mathrm{cg}=$ cultriform groove $; \mathrm{ci}=$ crista interfenestralis; $\mathrm{cp}=$ cultriform process of the basisphenoid; $\mathrm{cpr}=\mathrm{crista}$ prootica; $\mathrm{ct}=\mathrm{crista}$ tuberalis; $\mathrm{ctr}=$ caudal tympanic recess; $\mathrm{fm}=$ foramen magnum $; \mathrm{fo}=$ foramen ovale $; \mathrm{fp}=$ fenestra pseudorotunda icf $=$ internal carotid foramen; $1 . \mathrm{bpt}=$ left basipterygoid process; $1 . \mathrm{pop}=$ left paroccipital process of the opisthotic; $\mathrm{mcv}=$ foramen for the middle cerebral vein; $\mathrm{mp}=$ median process; $\mathrm{nc}=$ nuchal $\mathrm{crest} ; \mathrm{oc}=\mathrm{occipital}$ condyle; pit $=$ pituitary fossa; prtr = prootic divisin of the rostral tympanic recess; $r$.bpt $=$ right basipterygoid process; r.pop $=$ right paroccipital process of the opisthotic; $s c r=$ subcultriform recess; $s r t r=$ subotic division of the rostral tympanic recess; ssc $=$ semicircular canal; ssr $=$ subsellar recess; $V=$ opening for the trigeminal nerve; VI = foramen or canal for the abducens nerve; VII = foramen or canal for the facial nerve; VIII = foramen or canal for the vestibulocochlear nerve; IX = foramen or canal for the glossopharyngeal nerve; $\mathrm{X}=$ foramen or canal for the vagus nerve; XII = foramen or canal for the hypoglossal nerve.

thicker than the tapering posterior end (Fig. 22.3, 22.7, 22.10). The bone is anteroposteriorly widest along its proximal margin and the shaft tapers distally until the distal end expands. The proximal and distal ends of the fibula are symmetrical in lateral view. The anterior margin of the fibula is straight, but the posterior margin is more concave in lateral view. The anterior surface of the distal end has a short, straight ridge that terminates proximally where the shaft is thinnest (Figs. 22.4, 22.8, 22.12, 61.14, 61.16). A short, low S-shaped ridge extends down the anterolateral surface of the fibula and provides an insertion for the M. iliofibularis (Nesbitt, 2011; Fig. 22.1, 22.1, 22.8, 22.12). This tubercle is not as large as the curving flange found in ceratosaurians (Carrano and Sampson, 2008; Carrano et al., 2012).

A subtriangular rugosity abuts the proximal margin of the medial concavity that receives the fibular flange of the tibia (Fig. 22.2, 22.5, 22.9, 22.14). Some coelophysoids (Raath, 1977; Colbert, 1989; Rowe, 1989; Ezcurra, 2017; Griffin, 2018), Eodromaeus murphi (Martínez et al., 2011), and early averostrans (Madsen, 1976; Madsen and Welles, 2000) have a short ridge on the medial side of the proximal end, but Dilophosaurus wetherilli, Dracoraptor hanigani (Martill et al., 2016), and Liliensternus lilienterni do not (von Huene, 1934).

Astragalus.-The main body of the astragalus is mediolaterally wide where it articulates with the distal end of the tibia (Figs. 23.1, 23.7, 34.1, 34.5). The distal surface of the astragalus is smooth where it articulates with the distal tarsals, and it is concave in anterior view. The posterior margin is straight in proximal view and the anteromedial corner projects away from the rest of the bone. The anterior edge of the astragalus is concave in front of the ascending process (=dorsal process, Welles, $1984 ;=$ ascending flange, Nesbitt, 2011). A rounded tuberosity projects proximally from the posteromedial corner of the astragalus.

The most prominent feature on the astragalus is the ascending process, a pyramidal projection that emanates from the middle of the anterior half of the bone and slants laterally before terminating in a point (Figs. 23.1, 34.6). The ascending process is prominent; it is much taller relative to the rest of the astragalus than that of Coelophysis bauri (Colbert, 1989), Syntarsus kayentakatae (Rowe, 1989), and Segisaurus halli (Carrano et al., 2012), and it resembles the anterior process of Zupaysaurus rougieri (Ezcurra and Novas, 2007) and Cryolophosaurus ellioti (Smith et al., 2007). The proximal surface of the ascending process is thin and subrectangular where it articulates with the anterolateral process of the tibia (Figs. 23.3, 34.7), unlike that of early sauropodomorphs in which it is broad and elliptical (Cooper, 1981; Langer, 2003; Moser, 2004; Marsh and Rowe, 2018) and the plate-like ascending process of early averostrans (Madsen, 1976; Madsen and Welles, 2000).
Welles $(1954,1984)$ located what he determined to be a sutural contact around the base of the ascending process and the main body of the astragalus of the holotype specimen (Fig. 23.1, 23.7), and he used this to suggest that the astragalus actually comprises two centers of ossification. A large coelophysid from Petrified Forest National Park also preserves these two ossification centers (UCMP 129618/PEFO 21373), but in that specimen the astragalus and calcaneum are coossified (Padian, 1986).

Three ridges extend down the base of the ascending process of Dilophosaurus wetherilli in proximal view (Figs. 23.3, 23.9, 34.7). The first is the anterior ridge that forms the anteroproximal edge of the bone. A sharper posterolateral ridge extends from the top of the ascending process to the posterolateral corner of the astragalus, dividing the broad tibial facet from the smaller fibular facet. The shortest and steepest of the three ridges is the anterolateral ridge, which forms the anterior margin of the fibular facet. A subtriangular fossa is present between the anterior ridge and anterolateral ridge on the front of the astragalus. Two foramina are present in this fossa; one of the foramina (=extensor canal, Nesbitt, 2011) is large, circular, and is probably confluent with a circular hole on the posterior side the ascending process. Several smaller foramina are present on the anterodistal surface of the astragalus.

The tibial facet of the proximal surface of the astragalus is deepest right behind the ascending process; the entire basin receives the posterolateral process of the tibia. The fibular facet is complete when the calcaneum is articulated to the lateral surface of the astragalus. A shallow fossa occurs on the medial surface of the astragalus similar to that found on the astragali of Syntarsus rhodesiensis and Camposaurus arizonensis (Ezcurra and Brusatte, 2011).

Calcaneum.-The medial side of the calcaneum is slightly convex where it articulates with the lateral side of the astragalus. The top of the calcaneum is concave and makes up the lateral half of the fibular facet (Fig. 23.5, 23.11). The calcaneum is strongly convex distally in lateral view and slightly concave proximally. A shallow fossa is present behind a curved ridge on the lateral surface of the calcaneum. The astragalus and calcaneum are coossified in many neotheropods (Nesbitt and Ezcurra, 2015), but those of Dilophosaurus wetherilli, Cryolophosaurus ellioti, and Allosaurus fragilis are not (Madsen, 1976; Smith et al., 2007; Figs. 23, 34). Unlike that of Cryolophosaurus ellioti (Smith et al., 2007), the calcaneum lacks an articular facet for the lateral malleolus of the tibia. The proximal tarsals of coelophysoids are variably coossified to one another, even within a population of a single species (Raath, 1977; Colbert, 1989; Rowe, 1989; You et al., 2014; Ezcurra, 2017; Griffin, 2018). 


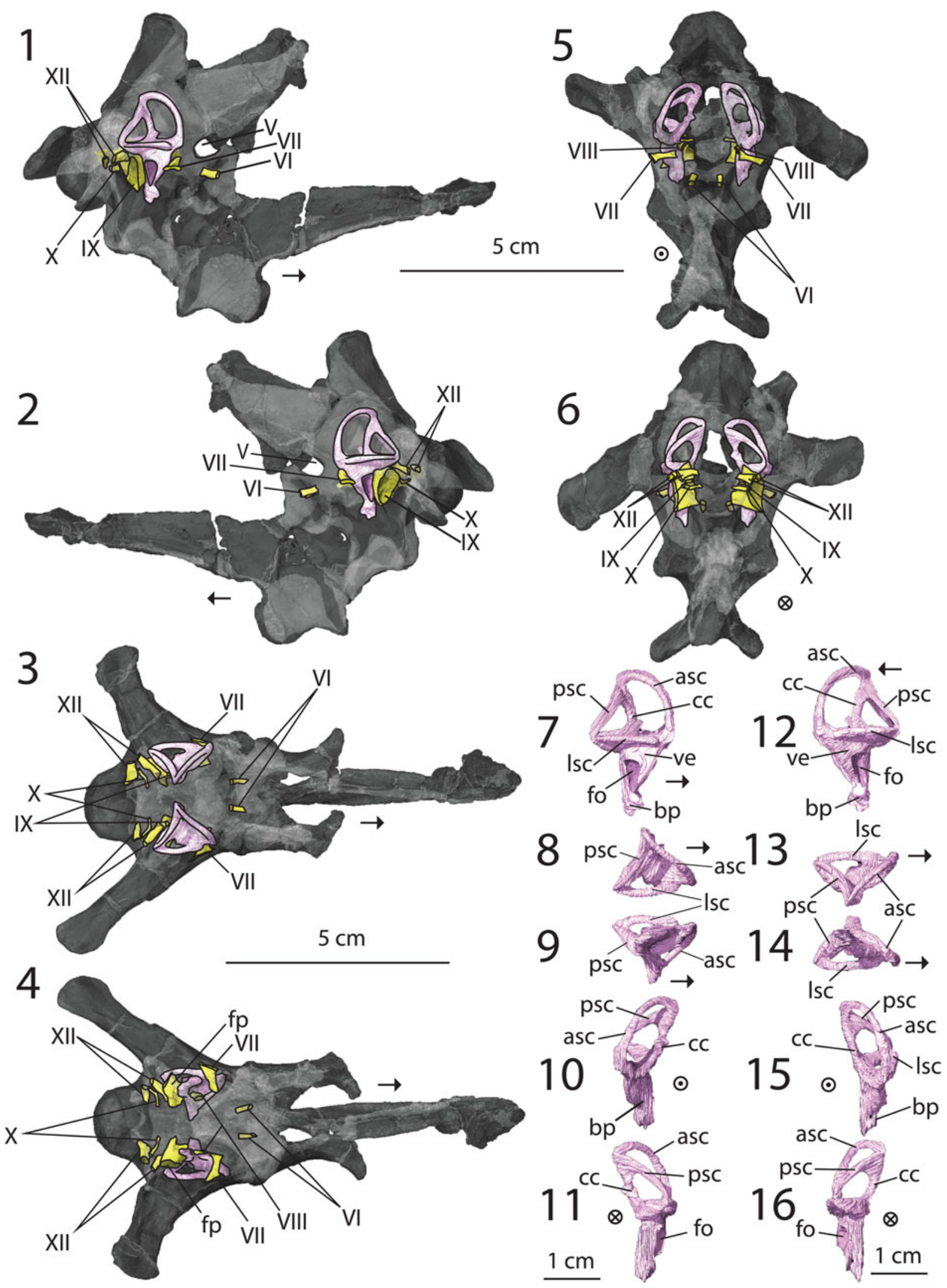

Figure 57. Dilophosaurus wetherilli referred specimen (TMM 47006-1): (1-6) articulated braincase showing segmented cranial nerve pathways and osseous labyrinths, (7-11) right osseous labyrinth, and (12-16) left osseous labyrinth in $(\mathbf{1}, \mathbf{7})$ right lateral, $(\mathbf{2}, \mathbf{1 2})$ left lateral, $(\mathbf{3}, \mathbf{8}, \mathbf{1 3})$ dorsal, $(\mathbf{4 , 9}, \mathbf{1 4})$ ventral, $(\mathbf{5 , 1 0} 15)$ anterior, and $(\mathbf{6}, 11,16)$ posterior view. Arrows point in anterior direction. asc $=$ anterior semicircular canal; $\mathrm{bp}=$ basilar papilla; $\mathrm{cc}=\mathrm{common}$ crus; fo $=$ foramen ovale; $\mathrm{fp}=$ fenestra pseudorotunda; $1 \mathrm{sc}=$ lateral semicircular canal; $\mathrm{psc}=$ posterior semicircular canal; ve = vestibule; $\mathrm{V}=$ opening for the trigeminal nerve; $\mathrm{VI}=$ foramen or canal for the abducens nerve; VII = foramen or canal for the facial nerve; VIII = foramen or canal for the vestibulocochlear nerve; IX = foramen or canal for the glossopharyngeal nerve; $\mathrm{X}=$ foramen or canal for the facial nerve; $\mathrm{XII}=$ foramen or canal for the hypoglossal nerve. 

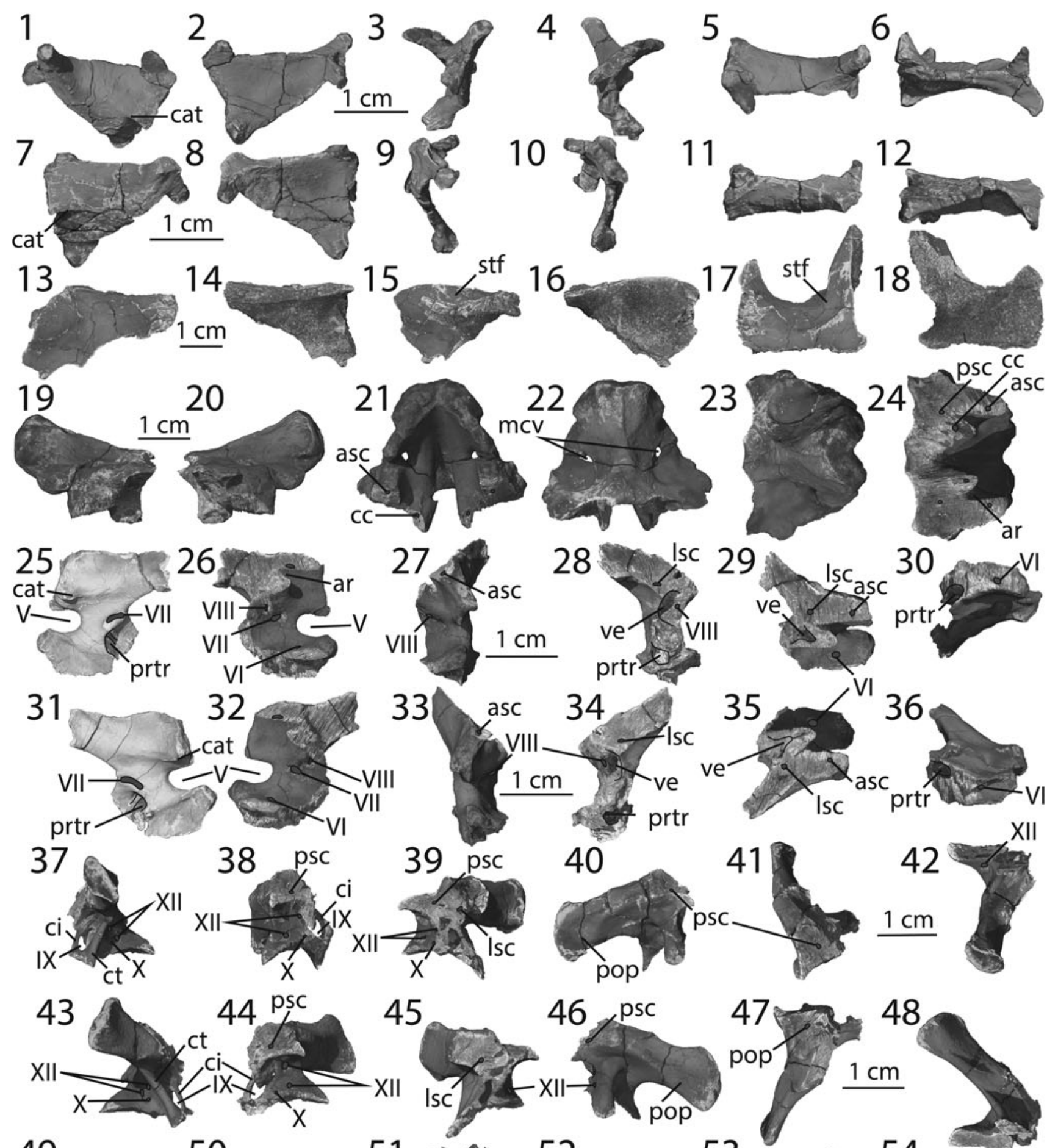

49

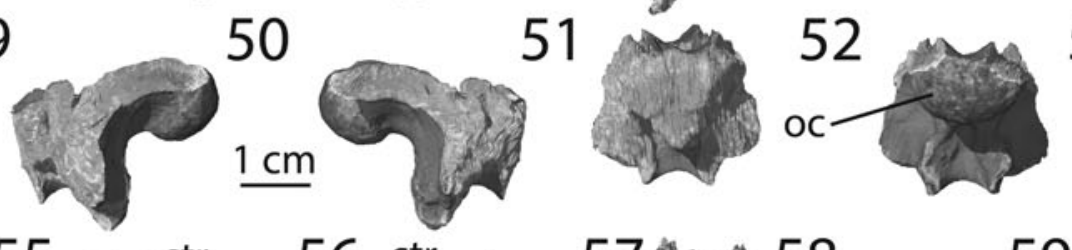

51
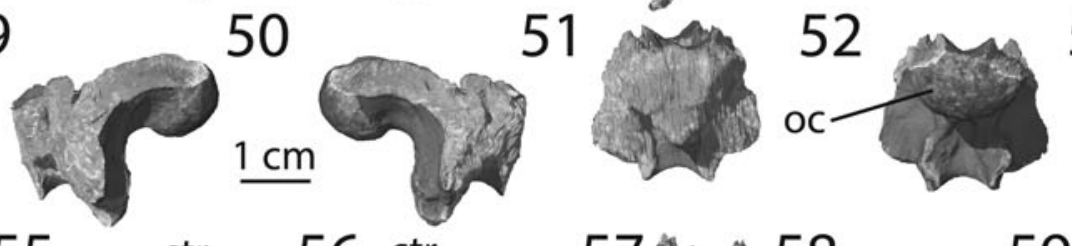

53

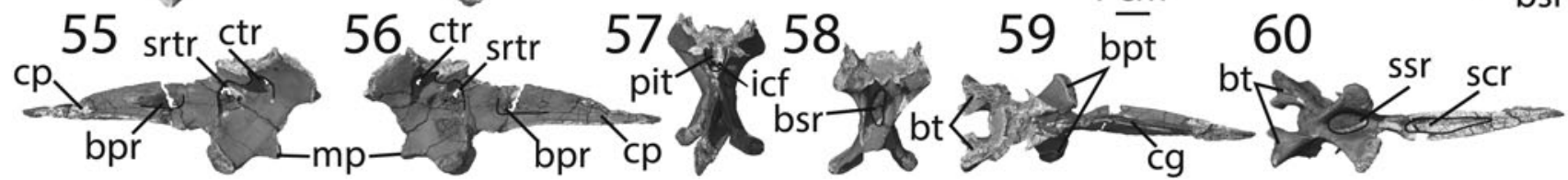

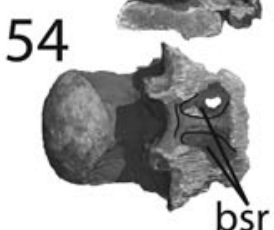


Figure 58. Dilophosaurus wetherilli referred specimen (TMM 47006-1): (1-6) left laterosphenoid, (7-12) right laterosphenoid, (13-18) left parietal, (19-24) supraoccipital, (25-30) left prootic, (31-36) right prootic, (37-42) left otooccipital, (43-48) right otooccipital, (49-54) basioccipital, and (55-60) parabasisphenoid (CT volume renderings with matrix digitally removed) in $(\mathbf{1}, \mathbf{1 3}, \mathbf{1 9}, \mathbf{2 5}, \mathbf{3 7}, \mathbf{4 9}, \mathbf{5 5})$ left lateral, $(\mathbf{7}, \mathbf{2 0}, \mathbf{3 1}, \mathbf{4 3}, \mathbf{5 0}, \mathbf{5 6})$ right lateral, $(\mathbf{2}, \mathbf{8}, \mathbf{1 4}, \mathbf{2 6}, \mathbf{3 2}, \mathbf{3 8}, \mathbf{4 4})$ medial, $(\mathbf{3}, 9$, $15,21,27,33,39,45,51,57)$ anterior, $(4,10,16,22,28,34,40,46,52,58)$ posterior, $(5,11,17,23,29,35,41,47,53,59)$ dorsal, and $(6,12,18,24,30,36,42,48,54$, 60 $)$ ventral view. ar = auricular recess; asc $=$ anterior semicircular canal; $b p r=$ basipterygoid recess; $b p t=$ basipterygoid; $b s r=b a s i s p h e n o i d$ recess; $b t=b a s a l$ tuber; cat $=$ crista antotica $\mathrm{cc}=$ common crus; $\mathrm{ci}=$ crista interfenestralis; $\mathrm{cg}=$ cultriform groove $; \mathrm{cp}=$ cultriform process of the basisphenoid; $\mathrm{ct}=\mathrm{crista}$ tuberalis; $\mathrm{ctr}=\mathrm{cau}-$ dal tympanic recess; icf $=$ internal carotid foramen; lsc = lateral semicircular canal; $\mathrm{mcv}=$ foramen for the middle cerebral vein; $\mathrm{mp}=$ median process; oc $=\mathrm{occipital}$ condyle; pit = pituitary fossa; pop = paroccipital process of the opisthotic; prtr = prootic division of the rostral tympanic recess; psc = posterior semicircular canal; scr $=$ subcultriform recess; str $=$ subotic division of the rostral tympanic recess; $\mathrm{ssr}=$ subsellar recess; $\mathrm{stf}=$ supratempral fossa; ve $=$ vestibule; $\mathrm{V}=$ opening for the trigeminal nerve; VI = foramen or canal for the abducens nerve; VII = foramen or canal for the facial nerve; VIII = foramen or canal for the vestibulocochlear nerve; $\mathrm{IX}=$ foramen or canal for the glossopharyngeal nerve; $\mathrm{X}=$ foramen or canal for the vagus nerve; $\mathrm{XII}=$ foramen or canal for the hypoglossal nerve.

Distal tarsals.-Distal tarsals 3 and 4 are present plesiomorphically in Dilophosaurus wetherilli (Figs. 24.45-24.50, 51). They articulate with the distal surfaces of the astragalus and calcaneum, but the positional relationship between the distal tarsals is hard to identify because they do not match the proximal shape of any of the metatarsals. Distal tarsal 4 is similar to that of coelophysoids in that it is flat, has a rounded medial process, subcylindrical posterior process, and a pointed lateral process (Padian, 1986; Rowe, 1989; Tykoski, 1998; You et al., 2014; Dal Sasso et al., 2018; Fig. 24.45-24.50). Distal tarsal 3 is rhomboidal and looks like that of sauropodomorphs and other early saurischians; one side is flat and the other side is gently rounded (Fig. 24.49, 24.50). In proximal view, one of the corners of distal tarsal 3 creates an acute angle, and two foramina pierce the edge of the bone next to that corner.

Metatarsus.-Digit III is the longest in the foot, followed by digit IV and then digit II (Figs. 24, 35, 51, 54). Only metatarsals II-V articulate proximally with the tarsus; metatarsal I is set much farther down on the ventromedial surface of metatarsal II. Metatarsal III is the longest element in the pes, and metatarsals II and IV are subequal in length. The proximal ends of metatarsals II-IV are twisted laterally relative to the distal ends. The longest metatarsal (metatarsal III) is longer than $50 \%$ of the tibia, unlike the relatively shorter third metatarsal of early averostrans such as Allosaurus fragilis (Madsen, 1976), Ceratosaurus nasicornis (Madsen and Welles, 2000), and Piatnitzkysaurus floresi (Bonaparte, 1986). The pedal digit formula is 2-3-4-5-0, confirmed in the articulated right pes of TMM 43646-1. Except for the unguals and penultimate phalanges on each digit, every pedal phalanx has a pair of short proximal ventrolateral and ventromedial flexor ridges.

Metatarsal I is very short and does not articulate with the tarsus (see TMM 43646-1; Fig. 54.2, 54.4). Its proximal end tapers to a point, and the shaft is flattened and concave medially (Figs. 24.2-24.5, 24.24-24.27, 35.4-35.7, 54.2, 54.4). There are two rugosities on the distal end of the shaft; one short rugosity is present on the distolateral surface of the bone just before the distal condyles and the other rugosity is longer, wider, and found on the ventromedial surface of the bone. The distal condyles are separate structures ventrally, but transition into a smooth, bulbous distal articular end that projects dorsally. The distal end of metatarsal I is rounded (Figs. 24.22, 24.44, 35.3). It has an asymmetrical and dorsally projecting articular surface, which has a large medial ligament fossa and smaller lateral fossa. A tuberosity is present on the ventrolateral margin of each ligament fossa. A large, ligament fossa is found on the side of the lateral distal condyle.
Metatarsal II is trapezoidal in proximal view (Figs. 24.1, 35.14). The longer base of this trapezoid occurs on the lateral side, and the trapezoid is dorsoventrally elongate. The lateral surface of the proximal half of metatarsal II is flat where it articulates with metatarsal III. The rest of the shaft is round in crosssection and it tapers distally (Figs. 24.6-24.9, 35.10-35.13, 54.1-54.4). The distal end of metatarsal II is mediolaterally and dorsoventrally expanded and points distomedially. The dorsal margin of the distal condyles is continuous, forming a smooth, rounded surface. The distal condyles are separate ventrally and the lateral condyle is wider than the medial condyle (Figs. 24.22, 24.44, 35.15). Metatarsal II is asymmetrical in distal view because the ligament fossa on the side of the lateral condyle is much larger and deeper than that on the side of the medial distal condyle.

Metatarsal III is mediolaterally compressed proximally, but becomes rounded in cross section distally. Its proximal end is subrectangular and not hourglass-shaped (Figs. 24.1, 24.23, 35.20) similar to the tetanurans Piatnitzkysaurus floresi (Bonaparte, 1986) and Allosaurus fragilis (Madsen, 1976). When in articulation with the other metatarsals, the proximal end of metatarsal III does not back the ventral surface of the second and fourth metatarsals like the relatively narrower metatarsus of coelophysoids (Rowe, 1989; Tykoski, 1998, 2005) and Ceratosaurus nasicornis (Madsen and Welles, 2000; Nesbitt and Ezcurra, 2015). The shaft tapers distally and is slightly bowed outwards medially (Figs. 24.10-24.13, 24.32-24.35, 35.16-35.19, 54.1-54.4). The dorsal surface of the distal end is smooth dorsoventrally and expanded medially. The medial distal condyle is taller than the lateral distal condyle in distal view (Figs. 24.22, 24.44, 35.21). These condyles are not separated dorsally, but they are divided ventrally by a smooth groove. The lateral and medial ligament fossae are subequal in size and shape.

The long axis of metatarsal IV is convex laterally (Figs. 24.14-24.24, 24.36-24.39, 54.1-54.4). The proximal end is broad and flattened where the bone articulates with metatarsals III and V (Fig. 24.1, 24.23). The shaft does not taper much distally and remains a fairly constant thickness down its length. The distal outline of metatarsal IV is subtriangular, but its dorsal edge is convex (Fig. 24.22, 24.44). The distal articular surface is much narrower mediolaterally than that of metatarsals II and III. The distal end is rounded dorsally. The collateral ligament fossae are very shallow, but are dorsoventrally and proximodistally wide. The medial ligament fossa is larger than the lateral fossa.

The proximal articular surface of metatarsal $\mathrm{V}$ is subtriangular and flat (Fig. 24.1). The medial surface of the metatarsal 


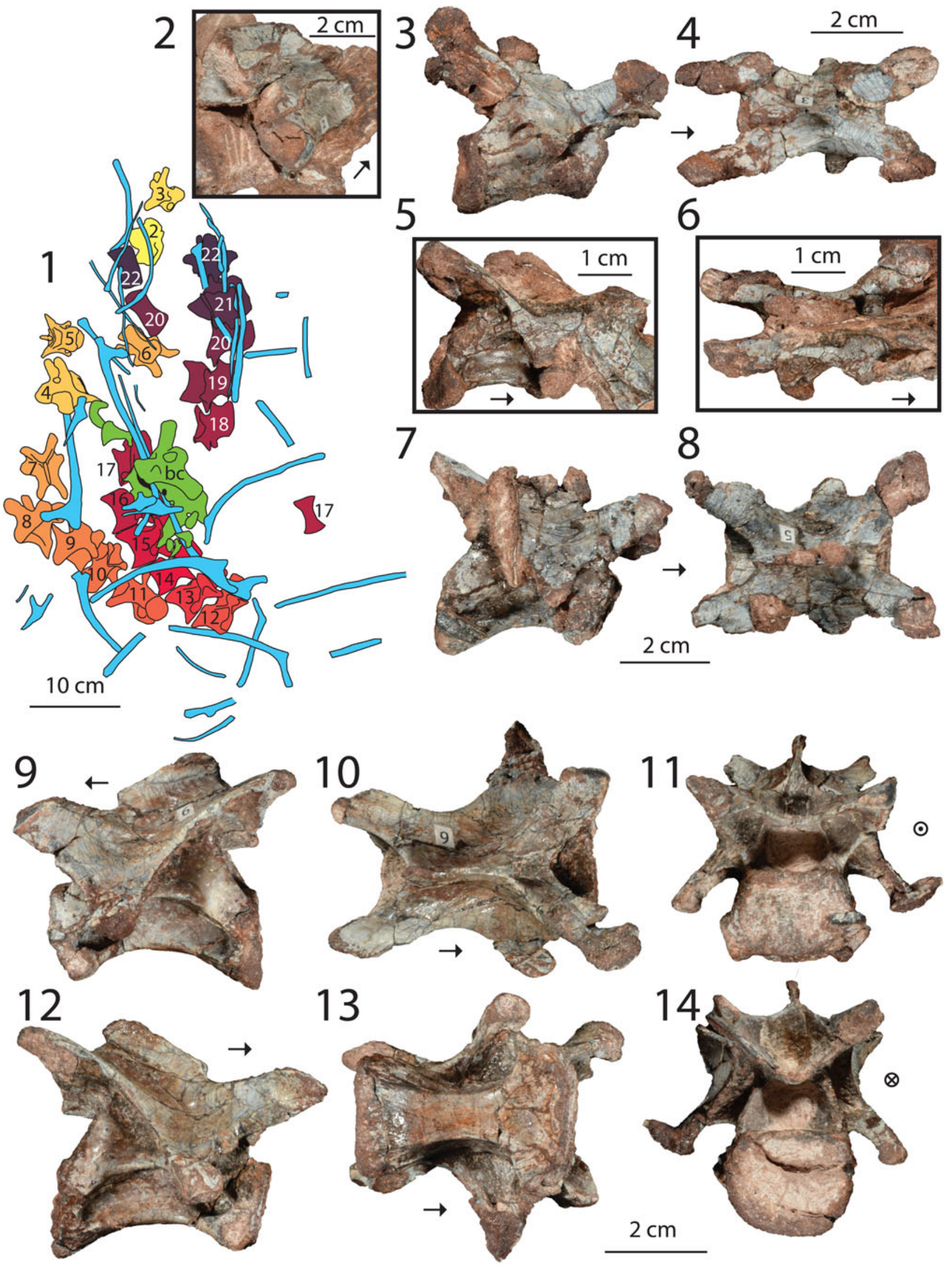

Figure 59. Dilophosaurus wetherilli referred specimen (TMM 47006-1): (1) map of presacral vertebrae as preserved, $(\mathbf{2})$ axis, $(\mathbf{3}, \mathbf{4})$ third cervical, $(\mathbf{5}, \mathbf{6})$ fourth cervical, $(\mathbf{7}, \mathbf{8})$ fifth cervical, and $(\mathbf{9 - 1 4})$ sixth cervical in $(\mathbf{2}, \mathbf{3}, \mathbf{5}, \mathbf{7}, \mathbf{1 2})$ right lateral, $(\mathbf{9})$ left lateral, $(\mathbf{4}, \mathbf{6 , 8}, \mathbf{1 0})$ dorsal, $(\mathbf{1 3})$ ventral, $(\mathbf{1 1})$ anterior, and $(\mathbf{1 4})$ posterior view. Arrows point in anterior direction. $b c=$ braincase. 

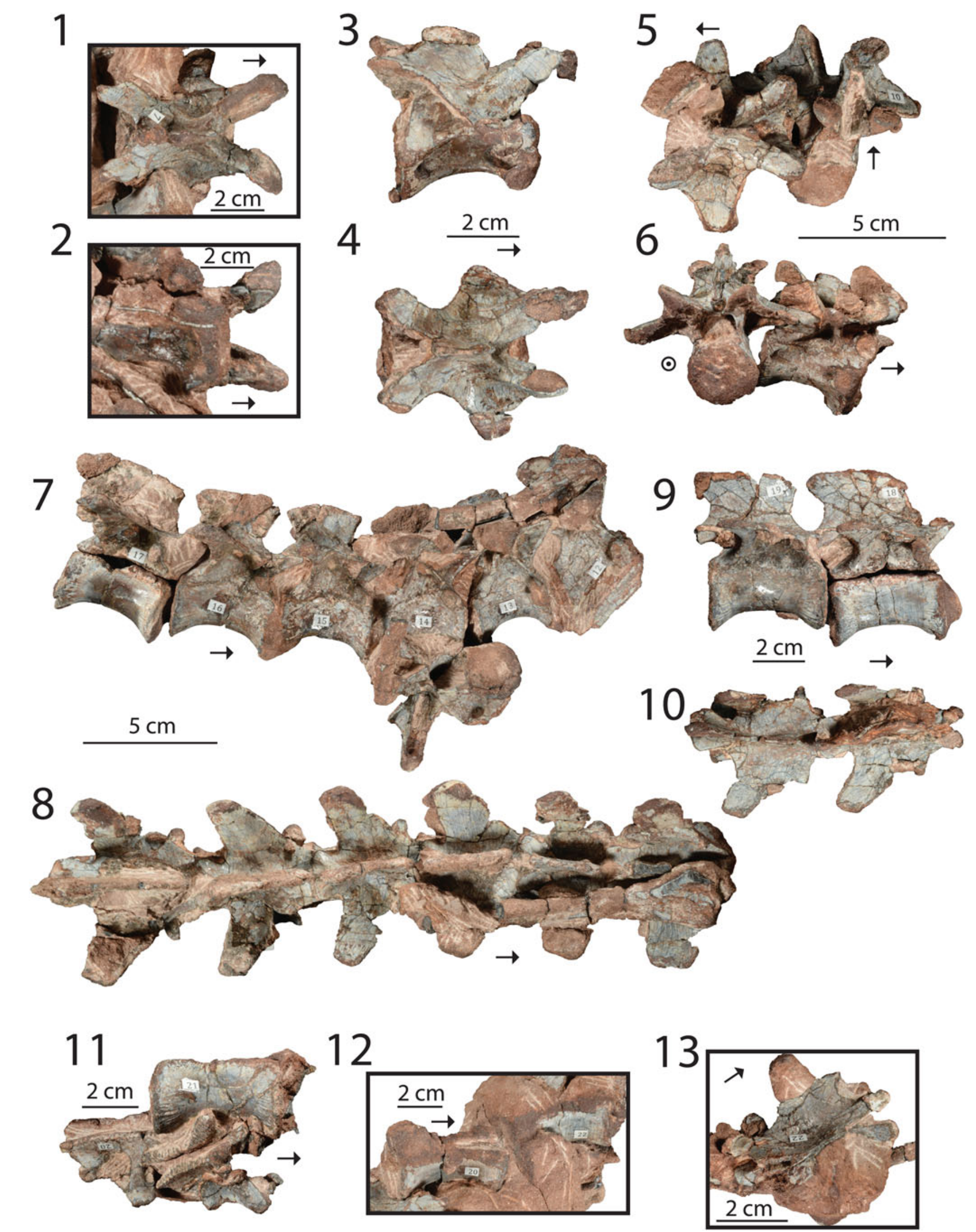

Figure 60. Dilophosaurus wetherilli referred specimen (TMM 47006-1): $(\mathbf{1 , 2})$ seventh cervical, $(\mathbf{3}, \mathbf{4})$ eight cervical, $(\mathbf{5}, \mathbf{6})$ ninth and tenth cervical, $(\mathbf{7 , 8}) 11$ th through 17 th vertebra, $(\mathbf{9 , 1 0})$ 18th and 19th vertebra, (11) 20th neural arch and 21st vertebra, (12) 20th and 22nd centra, and $(\mathbf{1 3}) 22 \mathrm{nd} \mathrm{neural} \mathrm{arch} \mathrm{in}(\mathbf{1 , 4 , 5 , 8 , 1 0 , 1 3 )}$ dorsal, $(\mathbf{2})$ ventral, $(\mathbf{3}, \mathbf{6}, \mathbf{7}, \mathbf{9}, \mathbf{1 1}, \mathbf{1 2})$ lateral, and $(\mathbf{6})$ posterior view. Arrows point in anterior direction. 


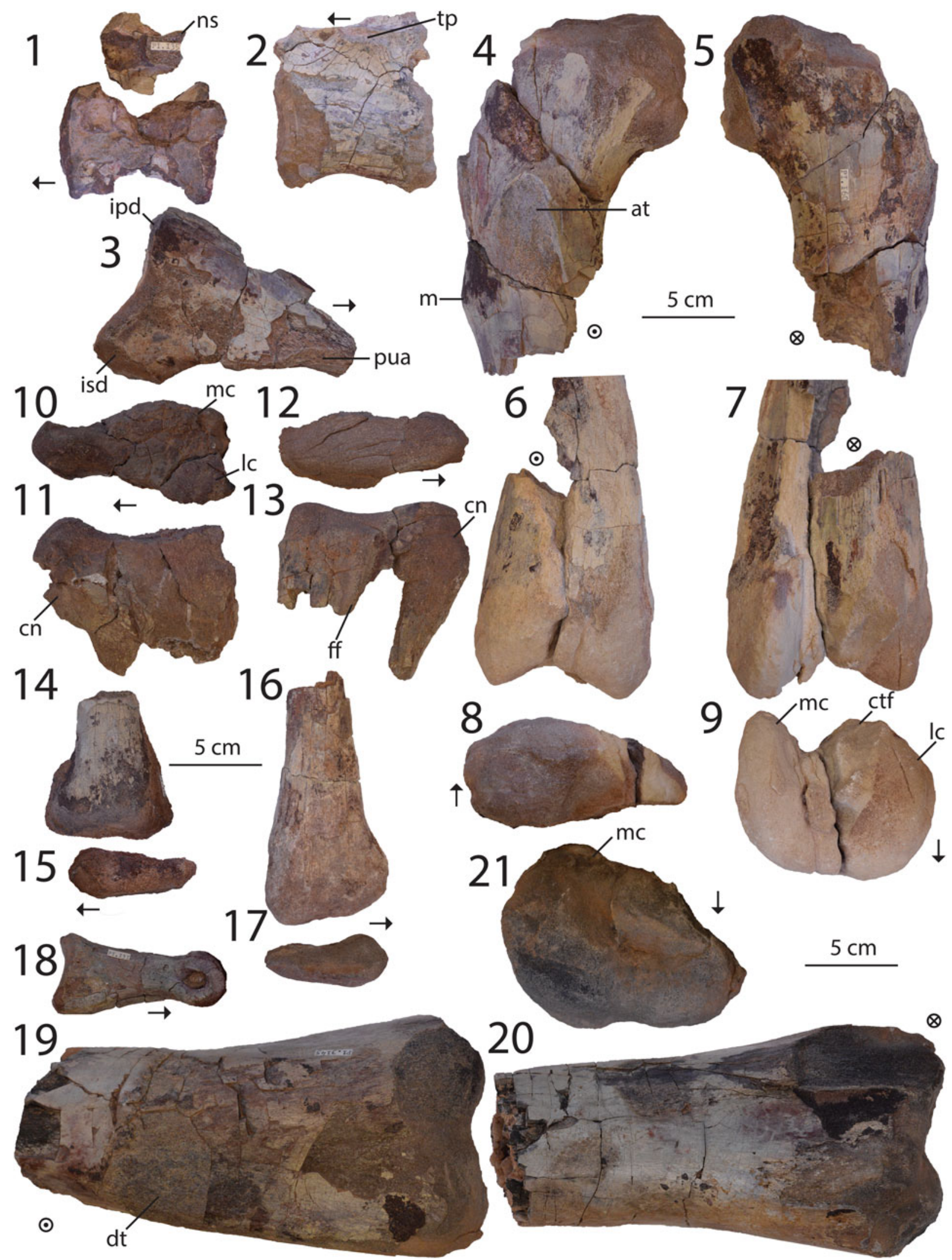

Figure 61. Specimens that are referred to cf. Dilophosaurus wetherilli including (1) cervical vertebra MNA V135, (2) anterior caudal vertebra MNA V177, (3) proximal end of left pubis MNA V154, (4-9) proximal and distal end of right femur MNA V160/V161,(10,11) proximal end of left tibia MNA V248, (12,13) proximal end of right tibia MNA V101, (14, 15) distal end of right fibula MNA V530, (16, 17) distal end of left fibula MNA V539, (18) pedal phalanx MNA V131, and $(\mathbf{1 9 - 2 1})$ distal end of right femur MNA V3145 in (1) dorsal, $(\mathbf{2}, \mathbf{1 1}, \mathbf{1 3}, \mathbf{1 4}, \mathbf{1 6}, \mathbf{1 8})$ lateral, $(\mathbf{3})$ medial, $(\mathbf{4}, \mathbf{6}, \mathbf{1 9})$ anterior, $(\mathbf{5}, \mathbf{7}, \mathbf{2 0})$ posterior, $(\mathbf{8}, \mathbf{1 0}, \mathbf{1 2})$ proximal, and $(\mathbf{9}, \mathbf{1 5}, \mathbf{1 7}, \mathbf{2 1})$ distal view. Arrows point in anterior direction. at $=$ anterior trochanger; $\mathrm{cn}=$ cnemial crest $; \mathrm{ctf}=$ crista tibiofibularis; $\mathrm{dt}=$ distal tuberosity; $\mathrm{ff}=\mathrm{fibular}$ flange $;$ ipd $=\mathrm{iliac}$ pedicle; isd = ischial pedicle; $\mathrm{lc}=$ lateral condyle; $\mathrm{m}=$ mound; $\mathrm{mc}=$ medial condyle; $\mathrm{ns}=$ neural spine; pua = pubic apron; $\mathrm{tp}=$ transverse process. 

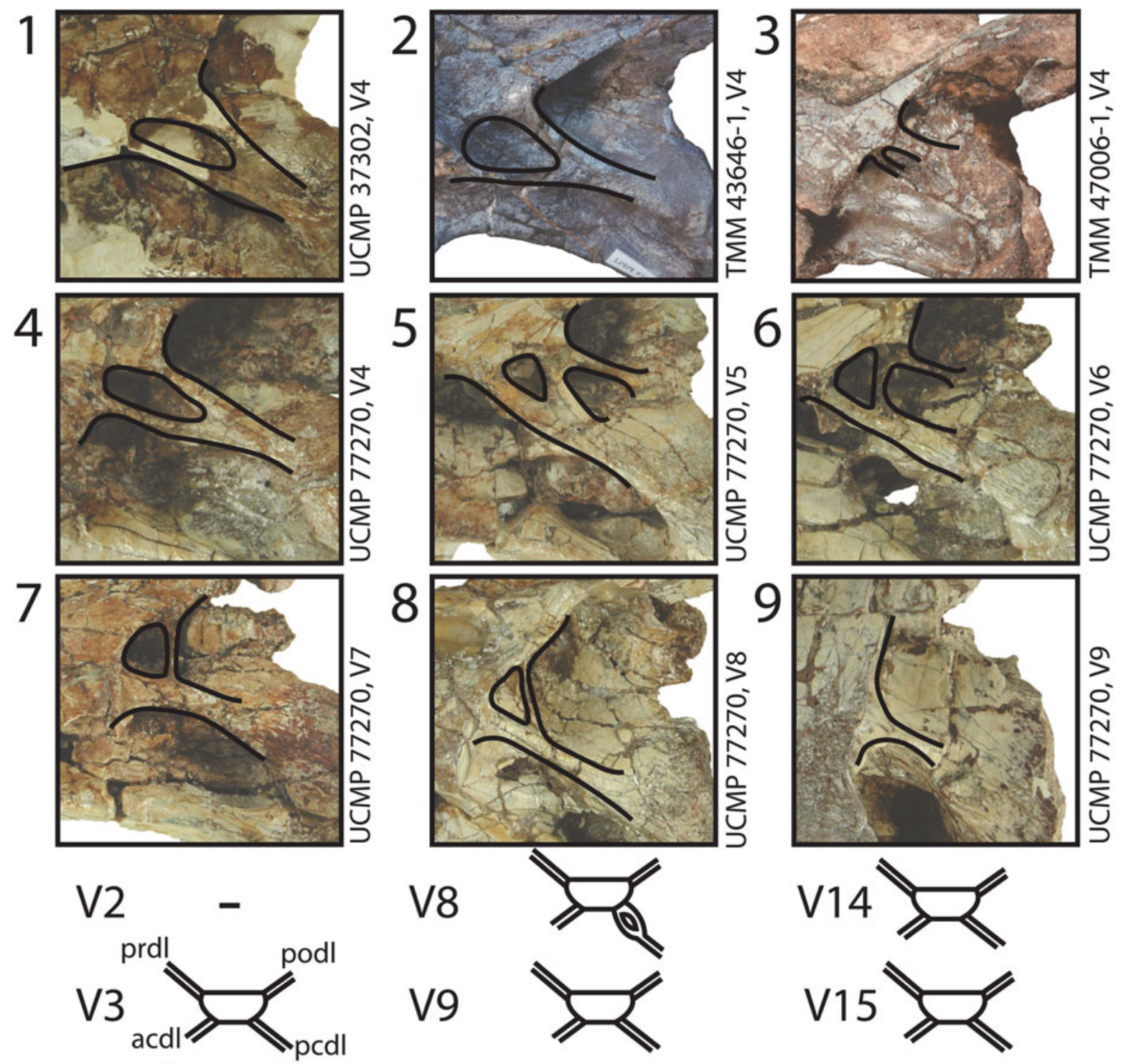

V9
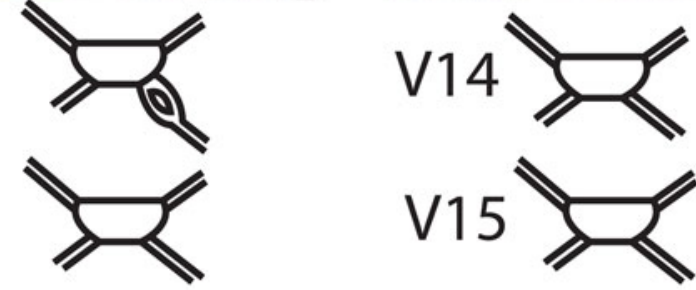

V4

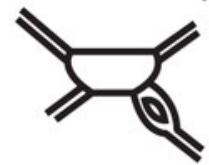

V10
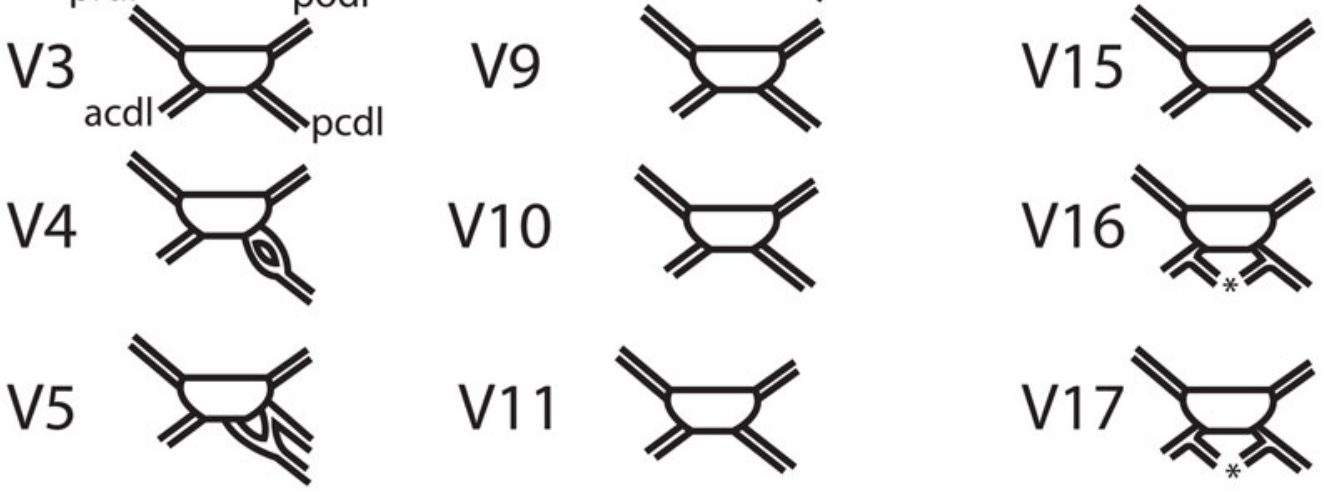

V6

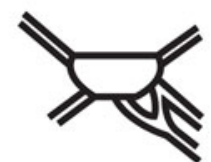

V12
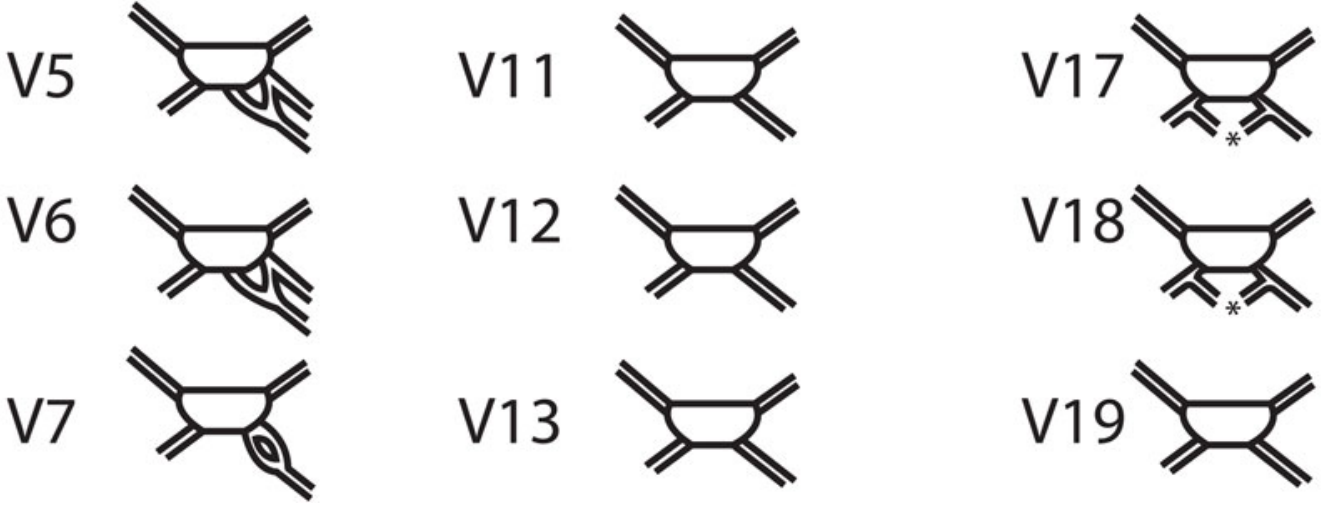

Figure 62. Serial changes in the posterior centrodiapophyseal lamina of the cervical vertebrae of Dilophosaurus wetherilli in left lateral view: (1) cervical vertebra four of UCMP 37302 (reversed), (2) cervical vertebra four of TMM 43646-1 (reversed), (3) cervical vertebra four of TMM 47006-1 (reversed), and (4-9) cervical vertebrae four through nine of UCMP 77270 ( $\mathbf{7}$ and $\mathbf{9}$ are reversed). Line drawings below depict the four basic vertebral laminae emanating from a trapezoidal diapophysis. The asterisk indicates accessory laminae described in the text. Anterior is to the left. $\mathrm{acdl}=$ anterior centrodiapophyseal lamina; pcdl $=$ posterior centrodiapophyseal lamina; podl = postzygadiapophyseal lamina; prdl = prezygadiapophyseal lamina; $\mathrm{v}=$ vertebra. 

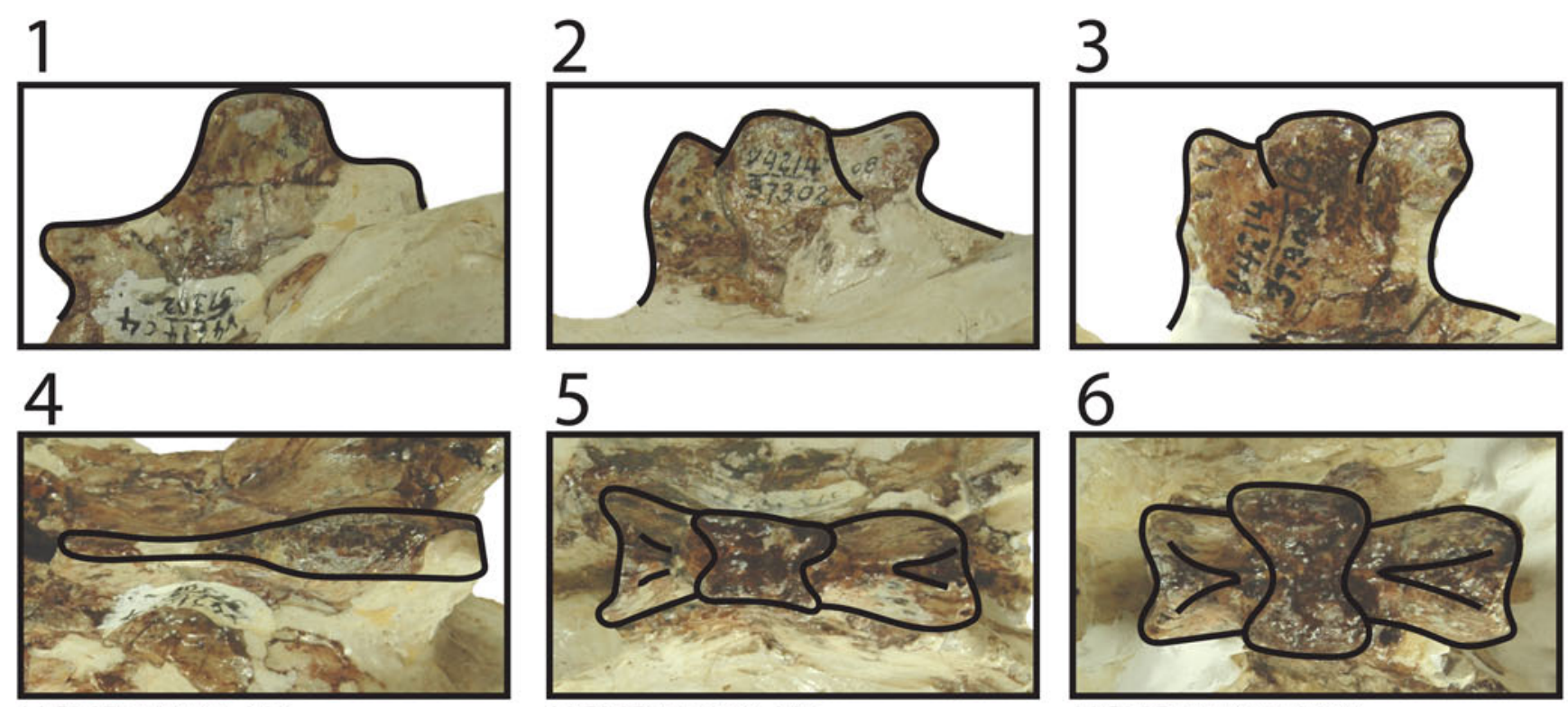

UCMP 37302, V4

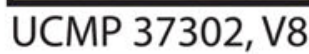

UCMP 37302, V10

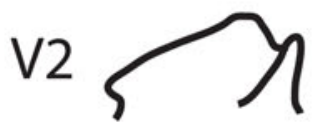

V3

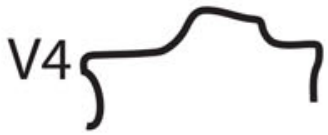
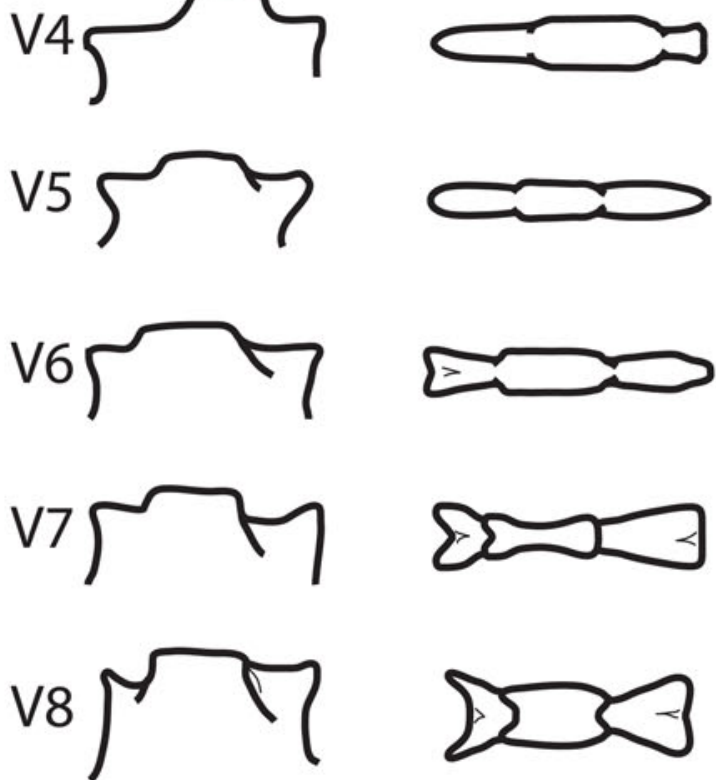

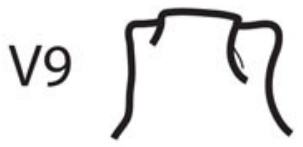
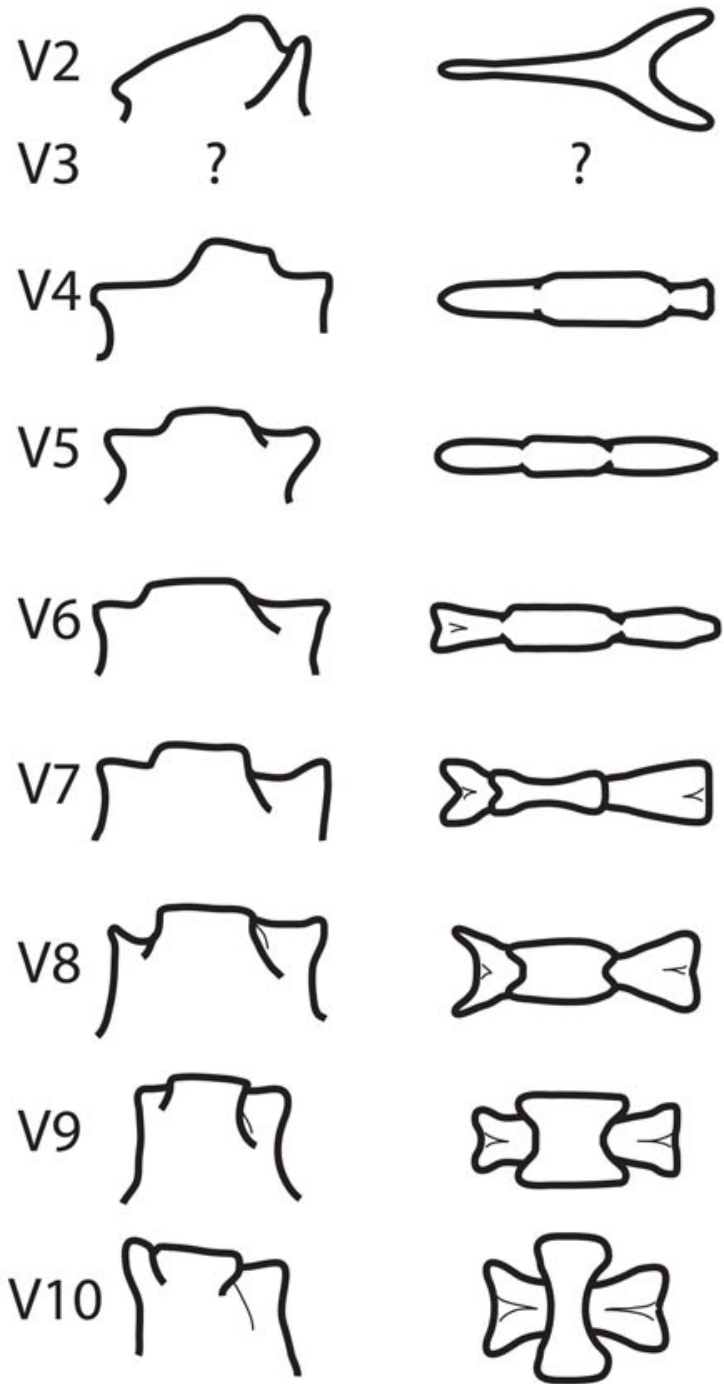

?
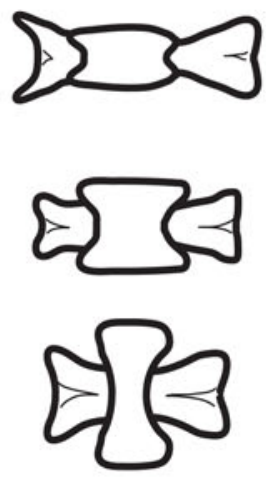
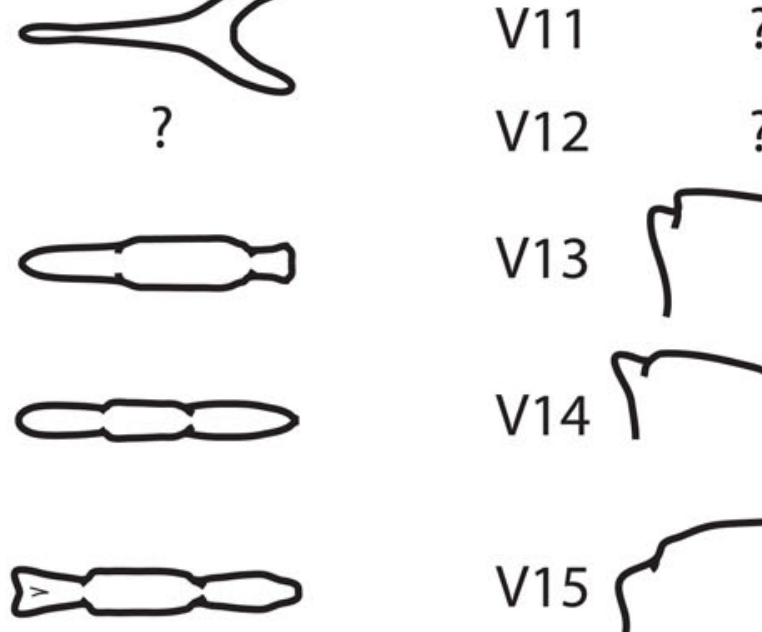

V12

?
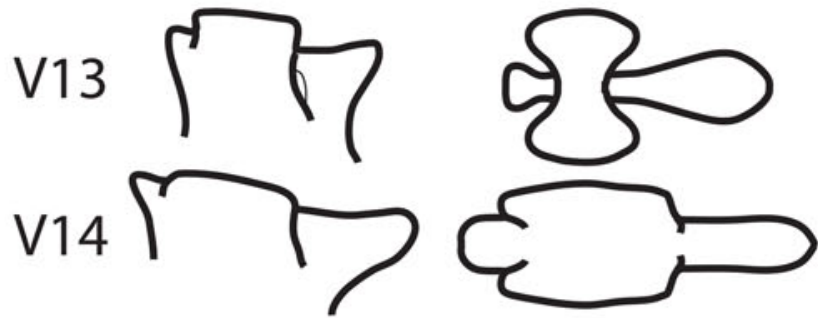

V15
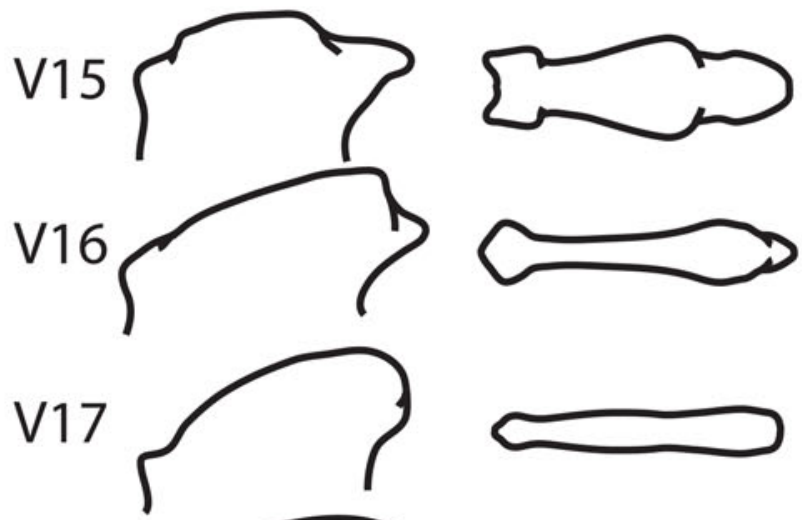

V18
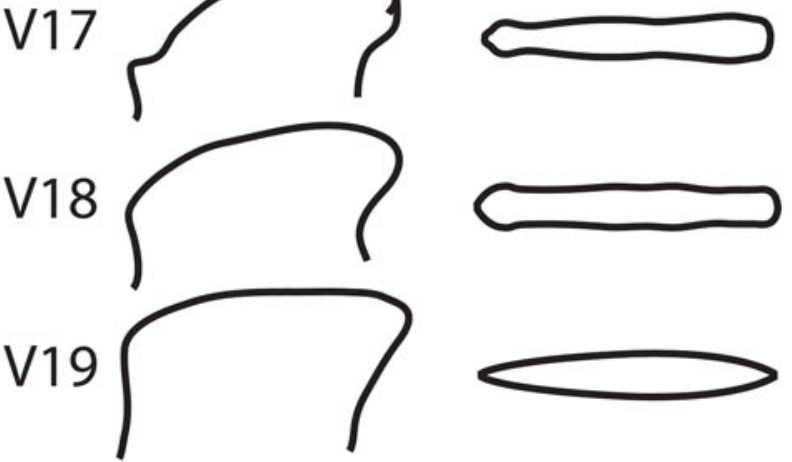

Figure 63. Serial changes in the neural spine of Dilophosaurus wetherilli in (1-3) left lateral view and (4-6) dorsal view: $(\mathbf{1}, \mathbf{4})$ cervical vertebra four of UCMP $37302,(\mathbf{2}, \mathbf{5})$ cervical vertebra eight of UCMP 37302 , and $(\mathbf{3 , 6})$ cervical vertebra ten of UCMP 37302. Line drawings below depict the neural spines in left lateral view (left) and dorsal view (right). Anterior is to the left for the line drawings. $v=$ vertebra 


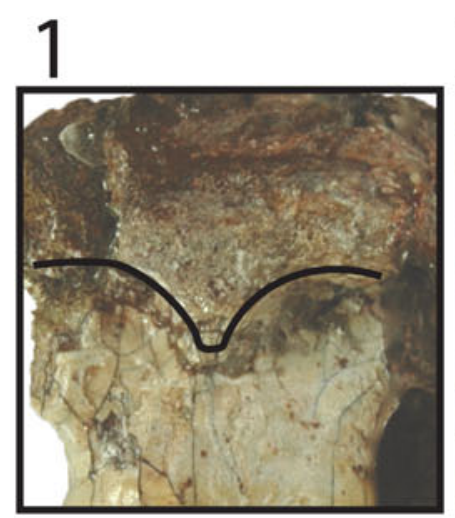

UCMP 77270, V4

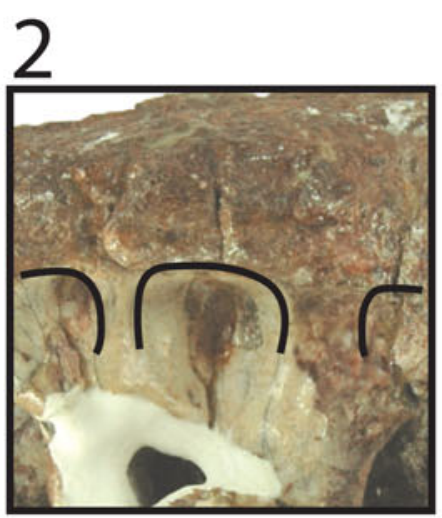

UCMP 77270, V5

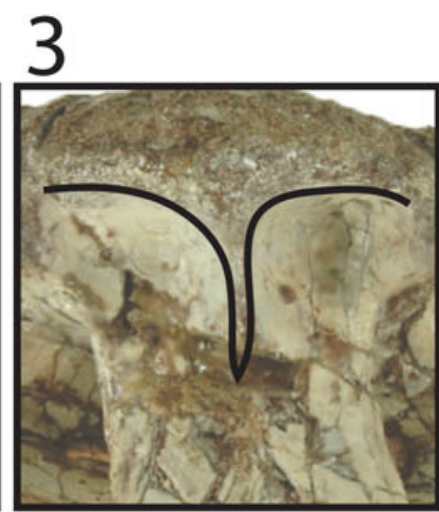

UCMP 77270, V9

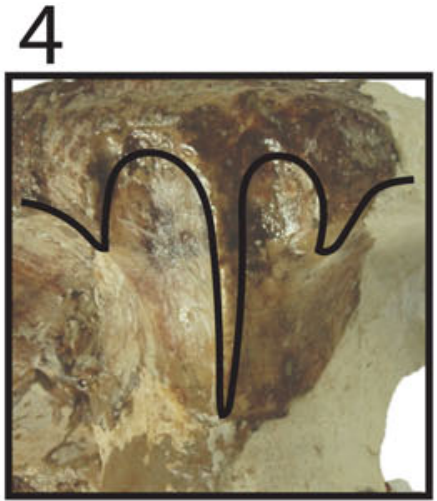

UCMP 37302, V12
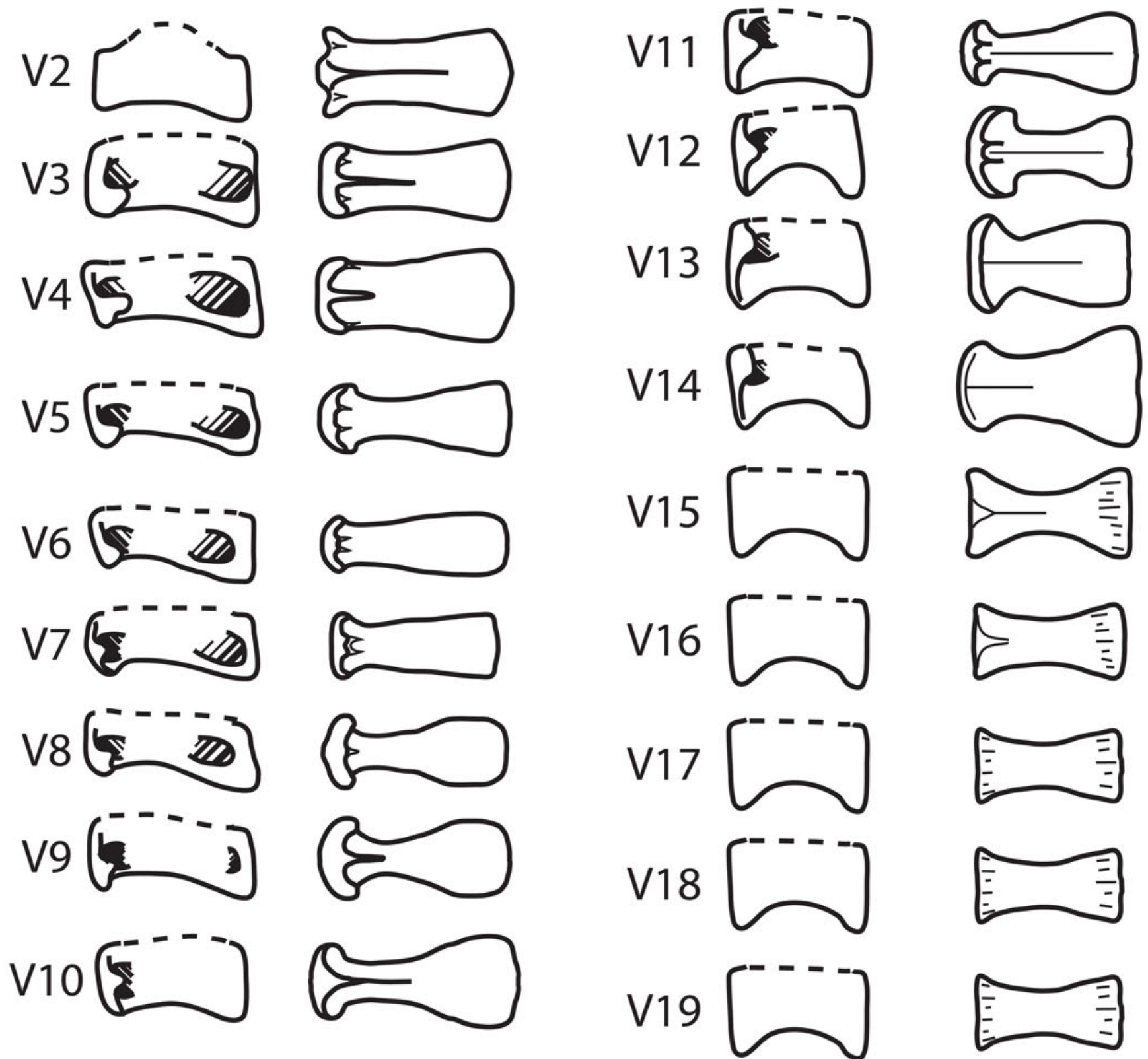

Figure 64. Serial changes in the centrum of Dilophosaurus wetherilli in ventral view: (1) cervical vertebra forur of UCMP 77270 , (2) cervical vertebra five of UCMP 77270, (3) cervical vertebra nine of UCMP 77270, and (4) cervical vertebra 12 of UCMP 37302 . Line drawings below depict the pleurocoels in left lateral view (left) and the ventral keel in ventral view (right). Anterior is to the left for the line drawings. $v=$ vertebra 
is concave where it articulates along its length with metatarsal IV. The bone tapers and curves down its length to a nonarticulating point (Figs. 24.18-24.21, 24.40-24.43, 35.22$35.25,54.1-54.4)$. There are two longitudinal ridges on the element; the first ridge begins at the top of the bone on its ventromedial surface and extends over to the dorsomedial surface, and the second ridge is present along the ventral edge of the bone.

Pedal phalanges.-Digit I: Phalanx I-1 is long and subcylindrical (Figs. 25.53-25.56, 54.2, 54.4). Its proximal end is concave and rounded, and it terminates at subsymmetrical distal condyles. The pedal ungual of digit I is slightly curved and is the smallest claw in the pes (Fig. 54.2, 54.4).

Digit II: Phalanx II-1 is the second-longest phalanx in the pes (Figs. 25.5-25.8, 25.57-25.60, 54.13-54.16). The proximal articular surface is smooth and concave. The dorsal margin is rounded in proximal view, but the ventral edge is concave and pointed at the ventral flexor ridges. The medial distal condyle is slightly taller than the lateral distal condyle, both have ligament fossae, and they are separated by a vertical groove along the distal surface. Shallow extensor pits are present on the dorsal surface behind the distal condyles. II-2 is divided proximally into lateral and medial articular surfaces by a vertical ridge that extends below the laterally-inclined, pointed extensor tubercle. The distal condyles are separate and symmetrical. The ungual on the second digit (II-3) is the longest in the foot (Figs. 25.13-25.16, 25.65-25.68, 54.13-54.16). The degree of curvature in the pedal claws is much less than that found in the manus. Proximally, II- 3 is triangular and is topped by a pointed extensor tubercle. The flexor tubercle is a roughened patch of bone in front of the proximoventral surface in all of the pedal unguals. Grooves extend down both sides of the distal end of the ungual.

Digit III: Phalanx III-1 is the longest element in the pes, apart from the metatarsals (Figs. 25.17-25.20, 25.69-25.72, 54.9-54.12). This bone is rounded and concave proximally. The lateral flexor ridge is more pronounced than the medial ridge. The distal end of the bone expands mediolaterally and is subequal in width to the proximal end. A shallow, elliptical extensor pit is on the dorsal surface at the far end of the bone. In distal view, the medial edge is straight, but the lateral edge is slanted ventromedially. The distal condyles are not divided by anything except a very slight ventral concavity. Deep, oblong ligament fossae are present on the side of both distal condyles. Phalanx III-2 is similar in shape to III-1, but it is dorsoventrally and proximodistally shorter. The third phalanx of digit III is subtriangular in proximal view and a groove divides the distal condyles along their entire surface. The ungual of digit III is divided proximally into two articular surfaces. Longitudinal medial and lateral grooves are more pronounced the ungual of digit III than the ungual of digit II (Figs. 25.29-25.32, 25.8125.84, 54.9-54.12).

Digit IV: The first phalanx of digit IV is subtriangular proximally and the dorsal apex leans laterally. The rest of this bone resembles phalanx III-2, but the distal condyles are more separated dorsally (Figs. 25.33-25.36, 25.85-25.88, 54.5-54.9). A circular extensor pit is present on the distal dorsal surface. The medial and lateral ligament fossae are wide and long. The second, third, and fourth phalanges of digit IV are similar in shape and get proportionally smaller down the digit. These phalanges have triangular proximal articular surfaces that are divided into medial and lateral halves. The shafts do not taper much, and the distal condyles are divided and have small dorsal extensor pits behind them. The fifth phalanx of digit IV is a gently curved ungual (Figs. 25.49-25.52, 25.101-25.104, 54.5-54.8).

\section{Phylogenetic results}

Quantitative results of the analyses are provided in Table 1. The holotype-only analysis recovered 12 most parsimonious trees (MPTs) of length 1191. When the paratype (UCMP 37303), TMM 43646-1, and TMM 47006-1 were iteratively paired in an analysis, the same 12 MPTs were found that are 2-5 steps longer. In the UCMP $37302+$ UCMP 77270 pair analysis, 18 MPTs were found with 1205 steps. In the all-specimen analysis, 83 MPTs were found of length 1206, one step longer than the UCMP 37302 + UCMP 77270 analysis.

All of the analyses resulted in the same topology of the nontheropod archosauromorphs included in the data matrix, so the following results will only focus on the theropod dinosaurs in the analyses. Dilophosaurus wetherilli is found as the sister taxon to Averostra when only the holotype specimen is scored in the matrix (Fig. 65.1). It falls within a grade of large nonaverostran neotheropods such as Cryolophosaurus ellioti and Zupaysaurus rougieri that, along with Coelophysoidea and Averostra, make up Neotheropoda. Liliensternus liliensterni and Lepidus praecisio are sister taxa and are either in a polytomy with Coelophysoidea or are basal coelophysoids in the strict consensus tree (Fig. 65). Syntarsus kayentakatae is the sister taxon to Coelophysidae, which is formed by Coelophysis bauri as the sister taxon to a clade including Syntarsus rhodesiensis and Camposaurus arizonensis. Eodromaeus murphi is found as the sister taxon to Neotheropoda, Chindesaurus bryansmalli, and Tawa hallae form a clade, and Herrerasauridae (comprising Herrerasaurus ischigualastensis

Table 1. Results of the six phylogenetic analyses conducted in this study, including the number of MPTs, length, CI, and RI. Absolute bootstrap and Bremer support values correspond to the node representing the Dilophosaurus wetherilli (Welles, 1954) lineage (shaded gray in Fig. 65).

\begin{tabular}{|c|c|c|c|c|c|c|}
\hline Analysis & MPTs & Length & $\mathrm{CI}$ & RI & Bootstrap (\%) & Bremer \\
\hline UCMP 37302 (holotype only) & 12 & 1191 & 0.368 & 0.696 & N/A & N/A \\
\hline UCMP 37302 + UCMP 77270 & 18 & 1205 & 0.363 & 0.690 & 84 & 3 \\
\hline UCMP 37302 + TMM 43646-1 & 12 & 1195 & 0.367 & 0.695 & 81 & 2 \\
\hline $\begin{array}{l}\text { UCMP } 37302 \text { + UCMP } 37303 \text { + UCMP } 77270 \text { + } \\
\text { TMM 43646-1 + TMM 47006-1 }\end{array}$ & 83 & 1206 & 0.363 & 0.690 & 72 & 2 \\
\hline
\end{tabular}



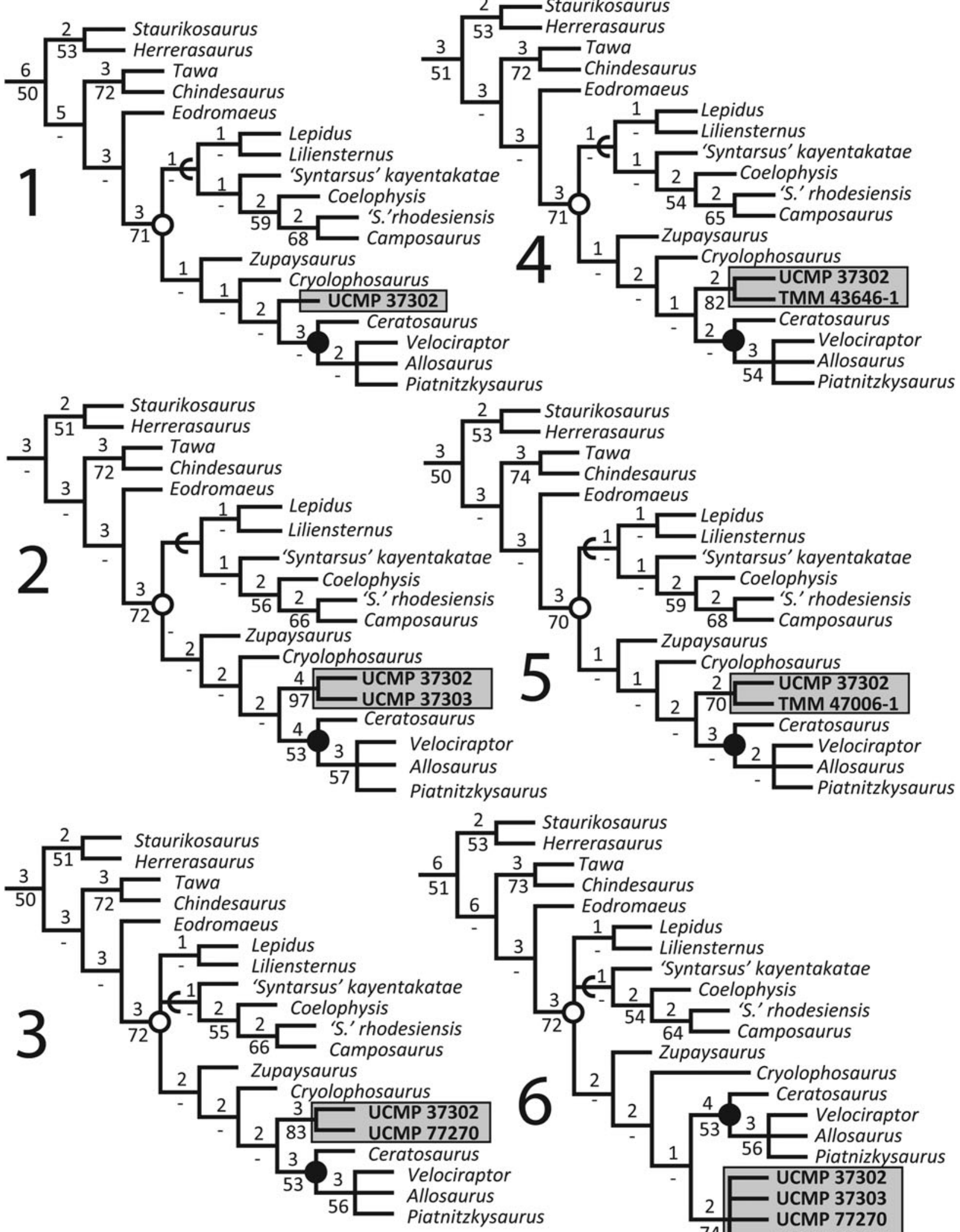

Staurikosaurus

Herrerasaurus

- Chindesaurus

Eodromaeus

3 - Liliensternus

'Syntarsus' kayentakatae

64 Camposaurus

$2 \longdiv { \text { Zupaysaurus } }$ Cryolophosaurus

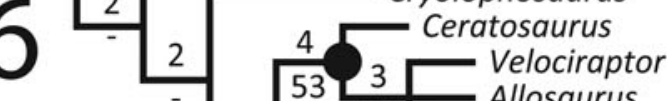

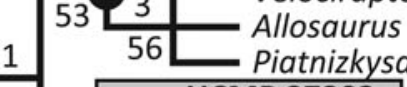

- UCMP 37302

2 UCMP 37303

74 UCMP 77270

TMM 43646-1

TMM 47006-1

Figure 65. Topological results of the phylogenetic analyses within Theropoda as strict consensus trees using (1) UCMP 37302, (2) UCMP 37302 and UCMP 37303, (3) UCMP 37302 and UCMP 77270, (4) UCMP 37302 and TMM 43646-1, (5) UCMP 37302 and TMM 47006-1, and (6) all UCMP and TMM specimens. Shaded boxes indicate specimen-level members of the Dilophosaurus lineage and node support is reported in Bremer values (above) and absolute bootstrap percentages (below). Open circles indicate Neotheropoda, closed circles indicate Averostra, and half-circles indicate Coelophysoidea. Dashes indicate bootstrap values less than 50\%. The number of MPTs, length, CI, and RI are reported in Table 1. 

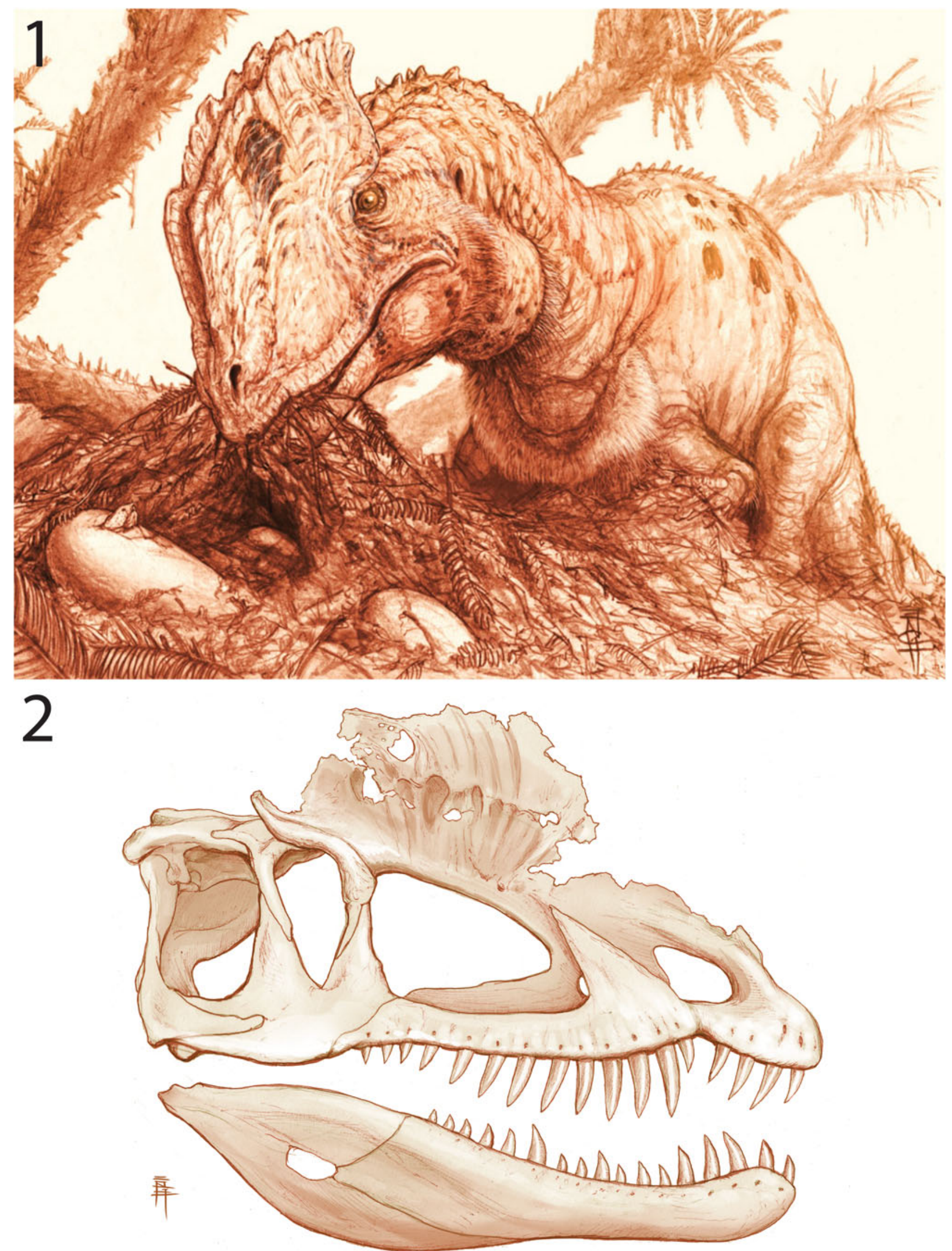

Figure 66. Reconstructions of Dilophosaurus wetherilli including (1) an adult Dilophosaurus wetherilli tending to its hatching clutch, and (2) cranial reconstruction of the skull and nasolacrimal crests from UCMP and TMM specimens in right lateral view. Art used with permission by Brian Engh (dontmesswithdinosaurs.com). 
and Staurikosaurus pricei) are the most basal group of theropods. The members of Tetanurae fall out in a polytomy in the strict consensus tree.

In the subsequent analyses (including the holotype specimen of Dilophosaurus wetherilli and one other referred specimen per analysis), all referred specimens from the UCMP and TMM are recovered as the sister taxon to the holotype specimen, UCMP 37302. This monophyletic 'Dilophosaurus wetherilli' is always found as the sister taxon to Averostra with Cryolophosaurus ellioti and Zupaysaurus rougieri assuming the same relationships with respect to one another and the rest of the theropod lineage (Fig. 65.2-65.5). Each of these analyses also recovers the same relationships as the holotype-only analysis of non-neotheropod theropods, including the relationships of members within. When all five UCMP and TMM specimens are included in the analysis, they form a monophyletic group (Fig. 65.6) as the sister taxon to Averostra.

The Bremer (Bremer, 1988, 1994) and absolute bootstrap (Felsenstein, 1985) support is low for stem-averostran theropods and coelophysoids in all of the analyses, but it varies from three to seven for Theropoda (Figs. 65.1-65.6). Bremer support for the 'Dilophosaurus' clade in the iterative analyses ranges from two to four (that for the holotype and paratype pair analysis is four). The node corresponding to the most recent common ancestor of all neotheropods has a bootstrap value of $71 \%$ when only the holotype is included, and this only varies up to $3 \%$ in the other analyses. The bootstrap support for the Tawa hallae + Chindesaurus bryansmalli clade varies between $72 \%$ and $74 \%$ in these analyses, and support for Theropoda varies from $<50 \%$ to $51 \%$. In the iterative pairs of Dilophosaurus specimens, bootstrap support varies between $70 \%$ and $97 \%$ (the holotype and paratype pair are $97 \%$ ), and when all of the specimens of Dilophosaurus wetherilli are included in the analysis, the monophyletic group they form has a bootstrap value of $74 \%$.

The holotype specimen of Dilophosaurus wetherilli has 29 autapomorphies found in all 12 MPTs of the holotype-only analysis and four additional apomorphies found in some of the MPTs of the iterative analyses (Supplemental Data). Ten of those 29 autapomorphies of the holotype specimen are found in all 83 MPTs of the all-specimen analysis, which also found 18 extra apomorphies of the 'Dilophosaurus' clade in some of the 83 MPTs of that analysis. A discussion of the characters unique to Dilophosaurus wetherilli is presented below.

\section{Discussion}

Revised diagnosis.-Welles (1954, 1970, 1984) described Dilophosaurus wetherilli before the general widespread use of phylogenetic methods in paleontology. Nevertheless, the unique combination of character states and autapomorphies found in our analyses support some of the features that were originally used to diagnose the taxon. Many features of Welles' diagnosis now appear to be plesiomorphic for Dilophosaurus within Neotheropoda. These include a large antorbital fenestra, a ventrally narrow orbit, a weak medial connection between the maxilla and premaxilla that are separated by a subnarial gap, a long posteromedial process of the premaxilla, four premaxillary teeth, a deep groove and low rugosae (interdental plates) in the maxilla that has 12 teeth, short cervical vertebrae that are planoconcave, deep 'chonoses' (pneumatic fossae) around the neural arch, hyposphene-hypantrum articulations between the eighth cervical and the sacrum, a humerus half of the length of the femur, a grasping hand with four digits, a tibia subequal in length to the femur, a foot with four functional toes, and digit I being reduced. Other features cited by Welles (1984) probably link Dilophosaurus to stem-averostrans. These include a preantorbital foramen in the maxilla within a maxillary recess, an expanded dorsal margin of the scapular blade, and a 'ceratosaurid' astragalus with a height:breadth ratio of 0.78 . Still other diagnostic features cited by Welles (1984) may reflect the differing levels of maturity at time of death in the specimens he studied. These include cervical ribs that are fused to the centra and the differential ossification of metatarsals I and V (Welles, 1984, claimed that metatarsal I is only ossified distally and metatarsal $\mathrm{V}$ is only ossified proximally).

We choose to diagnose Dilophosaurus wetherilli using the unambiguous autapomorphies and the unique combination of character states found when including only the holotype UCMP 37302 in our dataset. Yet, variations or other comments on the diagnosable features based on other specimens are also mentioned here.

Dilophosaurus wetherilli is the only theropod dinosaur to have these skeletal features: a pair of nasolacrimal crests on the skull (Fig. 66), and each one is formed by a ridge starting on the dorsolateral margin of the nasal process of the premaxilla (only preserved in the paratype UCMP 37303 and referred specimen UCMP 77270); dorsolateral expansions of the nasal and lacrimal; and a posteriorly projecting process of the lacrimal (cited as apomorphic by Welles, 1984 and Rauhut, 2003; Figs. 3.1-3.6, 26.6-26.10, 36, 43.9, 43.10); a pyramidal process on the lateral surface of the surangular in front of the articulation with the quadrate (Figs. 5.1, 5.2, 38, 45.5, 45.6); the mandibular fenestra is reduced in anteroposterior length (best preserved in UCMP 77270; Figs. 5.1-5.4, 38); the dorsal margin of the neural spines of the post-axial cervical vertebrae are stepped in lateral view, forming anterior and posterior 'shoulders' and a taller, central 'cap' that is cruciform in dorsal view, especially in more posterior cervical vertebrae (especially evident in UCMP 77270 and cited as apomorphic by Welles, 1984 and Rauhut, 2003; Figs. 7, 9, 39, 63); serial variation (bifurcation and recombination) exists in the posterior centrodiapophyseal laminae of the cervical vertebrae (especially evident in UCMP 77270; Figs. 7, 9, 39, 62); accessory laminae emanate from the anterior centrodiapophyseal laminae and posterior centrodiapophyseal laminae of the middle trunk vertebrae (also present in TMM 47006-1; Fig. 62); the first primordial sacral rib articulates with the preacetabular process of the ilium (Figs. 11, 40); an additional 'horizontal buttress' is present on the posteroventral portion of the coracoid next to the biceps tuber (also found in TMM 43646-1; Figs. 13.7, 13.8, 13.16, 13.18, 29.6-29.9, 29.14, 29.16, 29.17).

Additionally, Dilophosaurus wetherilli exhibits a unique combination of character states: the posterior end of the jugal terminates posterior to the infratemporal fenestra (shared with 
Velociraptor mongoliensis Osborn, 1924 in this matrix); the angle between the ascending process and longitudinal axis of jugal is $<75^{\circ}$ (shared with Eodromaeus murphi, Velociraptor mongoliensis, and coelophysids); ventral process of squamosal tapers ventrally (shared with coelophysids and Velociraptor mongoliensis); squamosal-quadratojugal contact absent (shared with coelophysids); angular extends farther posteriorly than surangular in the back of the jaw (shared with Zupaysaurus rougieri and Syntarsus kayentakatae); posterior pleurocoels on the lateral sides of the cervical centra (shared with coelophysoids and cited as apomorphic by Welles, 1984); rounded distal expansion of trunk neural spines in lateral view (shared with Herrerasaurus ischigualastensis); first primordial sacral rib circular in lateral view (reversal to plesiomorphic state for Dinosauria); prezygapophyses of distal caudal vertebrae not elongate (shared with Chindesaurus bryansmalli and Syntarsus rhodesiensis); gastralia form extensive basket (reversal to plesiomorphic state for Dinosauria); scapula blade height less than three times its distal width (shared with Eodromaeus murphi); postacetabular process strongly expanded laterally (shared with coelophysids); dorsolateral trochanter of femur is rounded ridge (shared with Herrerasaurus ischigualastensis, Chindesaurus bryansmalli, and some coelophysoids); mediolateral scar on the anterior surface of distal end of femur (shared with Eodromaeus murphi); deep fossa present on the medial surface of the proximal end of the fibula (shared with Herrerasaurus ischigualastensis and some coelophysoids); astragalus and calcaneum not coossified to one another (reversal among neotheropods, shared with Allosaurus fragilis, but also may be ontogenetically variable in nonaverostran theropods; Griffin, 2018; Griffin and Nesbitt, 2019); shallow fossa on the medial surface of the astragalus (shared with Camposaurus arizonensis, Ceratosaurus nasicornis, and Allosaurus fragilis).

Phylogenetic position of Dilophosaurus wetherilli.-Despite the low support values for the monophyly of clades of early theropods, the overall topology does not change during the iterative analyses. This means that we can have some confidence in the relationships of the theropods included in the analysis, but we have yet to find the characters to support important nodes such as Neotheropoda and Averostra. Unlike many previous analyses, Dilophosaurus wetherilli is not recovered in our study as an early member of Coelophysoidea within Ceratosauria (Rowe and Gauthier, 1990; Tykoski, 1998, 2005; Holtz, 2000; Tykoski and Rowe, 2004) or as a non-averostran coelophysoid (Ezcurra and Novas, 2007; Carrano et al., 2012; Xing, 2012). Instead, Dilophosaurus wetherilli is supported as a stem-averostran more derived in the direction of Averostra than Cryolophosaurus ellioti and Zupaysaurus rougieri. This result reflects previous hypotheses that recovered taxa that were assigned previously to the Coelophysoidea, such as Dilophosaurus wetherilli, as paraphyletic group more closely related to averostrans (Carrano et al., 2002; Rauhut, 2003; Smith et al., 2007; Langer et al., 2014; Zahner and Brinkmann, 2019). The characters that previously pulled Dilophosaurus wetherilli up the tree into Ceratosauria are found to be either homoplastic or redistributed across the tree, thanks to the discovery of new fossils and reinterpretations. Characters that previously pulled Dilophosaurus wetherilli up the tree into Ceratosauria, for example, having a robust, pyramidal ascending process of the astragalus (Tykoski, 2005) or two pleurocoels on the post-axial cervical vertebrae (Holtz, 2000), or down the tree at the base of Coelophysoidea, such as the presence of a subnarial gap (Ezcurra and Novas, 2007), have been re-distributed.

We find no support for a second theropod taxon in the material that Welles collected and those that were collected subsequently. Welles believed that UCMP 77270 might possibly represent a separate genus, although his views vacillated back and forth over many years (S. Welles, personal communication, 1980, 1986). In his publication (Welles, 1984, p. 89), he cited "differences in the skull proportions, vertebrae, and especially the femur" as justification for excluding this specimen from his description. Our results supporting the monophyly of Dilophosaurus (including UCMP 77270) suggest that only one taxon is represented. The differences that impressed Welles may result from different degree of maturity at time of death of the various specimens, something that Welles failed to consider, at least in print. The differences in the skull proportions may also be artifacts of incompleteness of the holotype and paratype skulls. Similarly, apparent differences in the vertebrae may be attributed to the fragmentary nature of the holotype vertebral column. UCMP 77270 is the only specimen referred to Dilophosaurus that has a trochanteric shelf on the anterior surface of the femur. However, the trochanteric shelf has been shown to be ontogenetically variable within populations of other early theropods such as Coelophysis bauri and Syntarsus rhodesiensis owing to the development of the insertion of the M. iliofemoralis externus (Griffin, 2018). Other authors have suggested that UCMP 77270 and TMM 43646-1 represent the oldest and youngest individuals of a second species of Dilophosarus (Hendrickx and Mateus, 2014) based on separated maxillary interdental plates instead of the coossified plates of Welles' hypodigm specimens. Those authors dismissed intraspecific and ontogenetic variation without much comment, but evidence accumulated to date indicates that many of the early theropods that have been collected and described since Welles' time reflect a wide range of skeletal maturity at time of death, it should also be noted that the structure of the interdental plates in the holotype specimen is obstructed and cannot be observed. Moreover, the interdental plates of UCMP 77270 are not separate throughout the length of the maxilla; they are coossified between the sixth and eighth alveolus. The uncertain stratigraphic position of UCMP 77270 was also advanced in support of a second species of Dilophosaurus by speculating that the time incorporated by the deposition of the Kayenta Formation could encapsulate a speciation event (Hendrickx and Mateus, 2014). However, after locating the holotype quarry, measuring a section of the Kayenta Formation through the site, and reviewing historic field notes, we are confident that UCMP 77270 could not be separated stratigraphically from the holotype by more than a few meters. All of the UCMP specimens are from the very narrow stratigraphic interval exposed in the floor of Moenkopi Wash below Moenave, Arizona. Our dates and correlations of measured sections indicate that the Dilophosaurus specimens found at Gold Spring are higher in the section and younger than those from Moenkopi Wash. In light of our present sample, one species, Dilophosaurus wetherilli, appears to have survived for a considerable amount of time captured in the Kayenta Formation. 
Finally, none of our analyses recovers a monophyletic 'Dilophosauridae' of crested theropod dinosaurs (i.e., Charig and Milner, 1990; Madsen and Welles, 2000; Yates, 2005; Hendrickx et al., 2015). While we only considered one other theropod with a crest (Cryolophosaurus ellioti) and not Sinosaurus triassicus or Shuangbaisaurus anlongbaoensis, it should be noted that the presence of a nasolacrimal crest does not determine relationships in our analyses. Dilophosaurus wetherilli, Sinosaurus triassicus, and Monolophosaurus jiangi exhibit similar crest construction, incorporating the premaxilla, nasal, and lacrimal, and the midline nasolacrimal crest of Cryolophosaurus ellioti is unlike that of any non-avian dinosaur. Instead of being unique to a small group of stem-averostran theropods, elaboration of the lacrimal and nasal may be plesiomorphically present in Ceratosauria and Tetanurae, and is common in early averostran theropods. As was pointed out previously (Brusatte et al., 2010), the presence of a clade of crested early theropods is more than likely the outcome of character construction and coding. Cranial ornamentation such as these crests likely served an important role in species identification (Hopson, 1975; Molnar, 1977; Padian and Horner, 2014; Xing et al., 2015; Gates et al., 2016) or perhaps in intersexual or intrasexual selection as they do in some extant dinosaurs (Jones and Hunter, 1993, 1999; Angst et al., 2019).

With the exception of Syntarsus kayentakatae (Rowe, 1989), all of the dinosaurs known from the Kayenta Formation are the earliest representatives of their respective clades in North America: Dilophosaurus wetherilli (non-coelophysoid Neotheropoda), Sarahsaurus aurifontanalis (Sauropodomorpha; Rowe et al., 2011; Marsh and Rowe, 2018), and Scutellosaurus lawleri and 'Scelidosaurus' Owen, 1861 (Ornithischia; Colbert, 1981; Padian, 1989). The Early Jurassic was a period of time in Earth history that reflects a biotic recovery from a mass extinction following the emplacement of CAMP volcanism and the opening of the northern Atlantic Ocean (Marzoli et al., 1999; Schaltegger et al., 2008; Blackburn et al., 2013), during which certain terrestrial lineages, such as sauropodomorph and theropod dinosaurs, were evolutionarily experimenting with an increase in body size around the globe (Sookias et al., 2012; Benson et al., 2014; Griffin, 2019; Griffin and Nesbitt, 2019). Sauropodomorphs accommodated an increase in overall body size to facilitate bulk herbivory by transitioning from bipedalism to quadrupedal locomotion (Bonnan, 2003; Yates and Kitching, 2003; Yates et al., 2009; Benson et al., 2014; McPhee et al., 2014) and by lightening the axial skeleton with extensive air sacs (correlated with more elaborate vertebral laminae and fossae; Wilson, 1999, 2012; O'Connor, 2006; Wedel, 2006; Yates et al., 2011; Butler et al., 2012). The increased body size of the earliest large-bodied theropod dinosaurs (correlated with cranial elaboration; Gates et al., 2016) was presumably an outcome of macropredation (Benson et al., 2014) because they most likely relied heavily on their forelimbs for prey acquisition or manipulation (Welles, 1984; Burch, 2014; Senter and Juengst, 2016). Their modified axial skeletons reduced weight and increased strength (Wilson, 1999; Wilson et al., 2011, 2016), and signal early stages in the acquisition of the avian respiratory system (Gauthier, 1986).

Many of the features that differentiate Dilophosaurus wetherilli from earlier theropod dinosaurs such as herrerasaurids and coelophysoids may be associated with an increase in body size and macropredation among theropod lineages through the end of the Triassic and the beginning of the Jurassic, including the tall nasolacrimal crests, the elaboration of the posterior centrodiapophyseal lamina and distal ends of the neural spines of the cervical vertebrae, the presence of accessory laminae supporting the neural arches of the middle trunk vertebrae, a dorsoventrally elongate orbit, more robust anterior trochanter, and dorsoventrally taller ascending process of the astragalus. While Dilophosaurus wetherilli could pluck fish and small prey in the fluvial systems depositing the Kayenta Formation (sensu Welles, 1984), the premaxilla-maxilla articulation is much more robust than originally hypothesized, and there is no doubt that the strong forelimbs were able to aid in the grasping and manipulation of large-bodied prey during predation or scavenging (Senter and Juengst, 2016; Senter and Sullivan, 2019). This capacity is supported by the presence of partially articulated specimens of the early sauropodomorph Sarahsaurus aurifontanalis containing large bite marks alongside shed teeth and a skeleton of Dilophosaurus wetherilli within the same quarry (Rowe et al., 2011; Marsh and Rowe, 2018).

\section{Conclusion}

A comprehensive redescription of all of the known specimens of Dilophosaurus wetherilli provides one of the most thorough anatomical studies currently available for early theropod dinosaurs and contributes to a more complete understanding of the intraspecific and ontogenetic variation of the earliest largebodied dinosaur in North America. Including only the holotype specimen of Dilophosaurus wetherilli in a phylogenetic analysis of archosaurs and early saurischians, we determine that Dilophosaurus is a stem-averostran theropod rather than a member of Ceratosauria or Coelophysoidea. Analyses in which the referred specimens of Dilophosaurus wetherilli were iteratively included with the holotype support not only the monophyly of the genus but also the existence of a single species of Dilophosaurus, which persisted for a considerable span of time throughout the deposition of the Silty Facies of the Kayenta Formation. Many of the distinct anatomical features of the skeleton of Dilophosaurus wetherilli may be associated with an increase in body size within the theropod lineage across the Triassic-Jurassic boundary, and the early expression of avian features including a respiratory system whose air sacs influenced vertebral architecture, and distinctive cranial ornaments that may have facilitated species recognition as in many living birds.

\section{Acknowledgments}

We especially acknowledge the people of the Navajo Nation and their role in welcoming and facilitating paleontological research on their land over the last 100 years. Thanks to J. Willie, Jr. (relative of J. Williams, the Navajo man who found the hypodigm of Dilophosaurus wetherilli in 1940) and the Navajo tour guides at the track site near Moenave, AZ, who helped us relocate the type quarry. We express our gratitude to A. Zaman, B. Nesemeier, and R. Carlton of the Navajo Nation Minerals Department for their advice, assistance, support in issuing permits, and 
reviewing manuscripts for this research. Fieldwork conducted by the University of Texas at Austin on the Navajo Nation was conducted under permits from the Navajo Nation Minerals Department (dated February 25, June 4, July 2, and October 15, 1997; May 11, 1999; March 27, 2000; July 8, 2015; and October $11,2016)$ in a collaboration with the Navajo EcoScouts program. Any persons wishing to conduct geologic investigations on the Navajo Nation must first apply for and receive a permit from, P.O. Box 1910, Window Rock, AZ 86515 and telephone number (928) 871-6587. This work was funded by National Science Foundation grants NSF CNS-0709135, EAR-0948842, EAR-1160721, EAR-1258878, EAR-1561622; by the Geology Foundation of the Jackson School of Geosciences at the University of Texas; and by the Doris O. and Samuel P. Welles Research Fund at the University of California Museum of Paleontology. Thanks to P. Holroyd and M. Goodwin (UCMP), D. Gillette and J. Gillette (MNA), and M. Brown and C. Sagebiel (TMM) for access to specimens, locality data, photographs, and historical archives in their respective collections. We especially thank K. Padian (UCMP) for not only inviting access to collections but for inviting unprecedented access to UCMP 77270 for description. P. Holroyd graciously photographed the right metatarsals of UCMP 37302 in ventral view. M. Brown transcribed and provided W. Langston, Jr.'s preparation notes. We thank O. Alcobar, W. Amaral, C. Bell, D. Dufaeu, E. Gordon, F. Jenkins, Jr., T. Macrini, J. Merck, C. Schaff, and R. Tykoski for all their hard work during multiple seasons excavating Dilophosaurus material from the Kayenta Formation. Thanks to M. Brown, C. Jass, B. Rainey, R. Tykoski, E. Yarmer, and $\mathrm{K}$. Bader for preparing the TMM specimens. Thanks to C. Bell, J. Clarke, D. Stockli, H.-D. Sues, and B. Parker for revising an earlier draft of the manuscript, and to M. Brown, C. Sagebiel, B. Breeden, R. Irmis, S. Nesbitt, M. Stocker, R. Tykoski, C. Kirk, P. Holroyd, M. Goodwin, A. Poust, and K. Padian, S. Welles, E. Colbert, W. Langston, Jr., J. Gauthier, A. Charig, and M. Raath for helpful conversations and advice. This manuscript was greatly enhanced by reviews from M. Ezcurra, M. Langer, and N. Smith. C. Marsh, W. Tintor, E. Lessner, and C. Lash helped relocate the holotype quarry of Dilophosaurus wetherilli. Thanks to B. Engh (http://dontmesswithdinosaurs. com/) for permission to use his artwork in this work, which was funded by A. Milner and the St. George Dinosaur Discovery Site at Johnson Farm. The conclusions presented here are those of the authors and do not represent the views of the United States Government.

\section{Accessibility of supplemental data}

Data available from the Dryad Digital Repository: https://doi. org/10.5061/dryad.r7sqv9s81.

\section{References}

Abdala, V., Vera, M.C., and Ponssa, M.L., 2017, On the presence of the patella in frogs: The Anatomical Record, v. 300, p. 1747-1755.

Allain, R., Tykoski, R., Aquesbi, N., Jallil, N.-E., Monbaron, M., Russell, D., and Taquet, P., 2007, An abelisaurid (Dinosauria: Theropoda) from the Early Jurassic of the High Atlas Mountains, Morocco, and the radiation of ceratosaurs: Journal of Vertebrate Paleontology, v. 27, p. $610-624$.
Alonso, P.D., Milner, A.C., Ketcham, R.A., Cookson, M.J., and Rowe, T.B., 2004, The avian nature of the brain and inner ear of Archaeopteryx: Nature, v. 430 , p. 666-669.

Angst, D., Barnoud, J., Cornette, R., and Chinsamy, A., 2019, Sex and ontogenetic variation in the crest of Numida meleagris: implications for crested vertebrates: The Anatomical Record, v, 303, p. 1018-1034.

Arcucci, A.B., and Coria, R.A., 2003, A new Triassic carnivorous dinosaur from Argentina: Ameghiniana, v. 40, p. 217-228.

Barnett, C.H., and Lewis, O.J., 1958, The evolution of some traction epiphyses in birds and mammals: Journal of Anatomy, v. 92, p. 593-601.

Bell, C.J., Head, J.J., and Mead, J.I., 2004, Synopsis of the herpetofauna from Porcupine Cave, in Barnosky, A.D., ed., Biodiversity Response to Climate Change in the Middle Pleistocene: The Porcupine Cave Fauna from Colorado: Berkeley, University of California Press, p. 117-126.

Bell, C.J., Gauthier, J.A., and Bever, G.S., 2010, Covert biases, circularity, and apomorphies: a critical look at the North American Quaternary Herpetofaunal Stability Hypothesis: Quaternary International, v. 217, p. 30-36.

Bell, C.J., Brown, M.A., Dawson, M.R., and Lundelius, Jr., E.L., 2013, Wann Langston, Jr.- a life among bones: Earth and Environmental Science Transactions of the Royal Socierty of Edinburgh, v. 103, p. 189-204.

Benson, R.B.J., 2010, A description of Megalosaurus bucklandii (Dinosauria: Theropoda) from the Bathonian of the UK and the relationships of Middle Jurassic theropods: Zoological Journal of the Linnean Society, v. 158, p. 882-935.

Benson, R.B.J., Campione, N.E., Carrano, M.T., Mannion, P.D., Sullivan, C., Upchurch, P., and Evans, D.C., 2014, Rates of dinosaur body mass evolution indicate 170 million years of sustained ecological innovation on the avian stem lineage: PLOS Biology, v. 12, p. e1001854.

Bever, G.S., Brusatte, S., Carr, T.D., Xu, X., Balanoff, A.M., and Norell M., 2013, The braincase anatomy of the Late Cretaceous dinosaur Alioramus (Theropoda, Tyrannosauroidea): Bulletin of the American Museum of Natural History, v. 376, p. 1-72.

Bittencourt, J.D.S., and Kellner, A.W.A., 2009, The anatomy and phylogenetic position of the Triassic dinosaur Staurikosaurus pricei Colbert, 1970: Zootaxa, v. 2079, p. 1-56.

Blackburn, T.J., Olsen, P.E., Bowring, S.A., McLean, N.M., Kent, D.V., Puffer, J., McHone, G., Rasbury, E.T., and Et-Touhami, M., 2013, Zircon U-Pb geochronology links the end-Triassic extinction with the Central Atlantic Magmatic Province: Science, v. 340, p. 941-945.

Bonaparte, J.F., 1979, Dinosaurs, a Jurassic assemblage from Pagagonia: Science, v. 205, p. 1377-1379.

Bonaparte, J.F., 1985, A horned Cretaceous carnosaur from Patagonia: National Geographic Research, v. 1, p. 149-151.

Bonaparte, J.F., 1986, Les dinosaures (carnosaures, allosauridés, sauropodes, cetiosauridés) du Jurassique moyen de Cerro Cóndor (Chubut, Argentine): Annales de Paléontologie (Vert.-Invert.), v. 72, p. 247-289.

Bonaparte, J.F., 1999, Evolución de las vértebras presacras en Sauropodomorpha: Ameghiniana, v. 36, p. 115-187.

Bonaparte, J.F., Novas, F.E., and Coria, R.A., 1990, Carnotaurus sastrei Bonaparte, the horned, lightly-built carnosaur from the Middle Cretaceous of Patagonia: Natural History Museum of Los Angeles County Contributions in Science, v. 416, p. 1-42.

Bonnan, M.F., 2003, The evolution of manus shape in sauropod dinosaurs: implications for functional morphology, forelimb orientation, and phylogeny: Journal of Vertebrate Paleontology, v. 23, p. 595-613.

Bradley, A., Burch, S.H., Turner, A.H., Smith, N.D., Irmis, R.B. and Nesbitt, S.J., 2020, Sternal elements of early dinosaurs fill a critical gap in the evolution of the sternum in Avemetatarsalia (Reptilia: Archosauria): Journal of Vertebrate Paleontology, v. 39, p. e1700992.

Bremer, K., 1988, The limits of amino acid sequence data in angiosperm phylogenetic reconstruction: Evolution, v. 42, p. 795-803.

Bremer, K., 1994, Branch support and tree stability: Cladistics, v. 10, p. 295-304.

Bristowe, A., and Raath, M.A., 2004, A juvenile coelophysoid skull from the Early Jurassic of Zimbabwe, and the synonymy of Coelophysis and Syntarsus: Palaeontologia Africana, v. 40, p. 31-41.

Brochu, C.A., 2003, Osteology of Tyrannosaurus rex: insights from a nearly complete skeleton and high-resolution computed tomographic analysis of the skull: Journal of Vertebrate Paleontology, v. 22 (Memoir 7), p. 1-138.

Brusatte, S.L., Benson, R.B.J., Currie, P.J., and Zhao, X., 2010, The skull of Monolophosaurus jiangi (Dinosauria: Theropod) and its implications for early theropod phylogeny and evolution: Zoological Journal of the Linnean Society, v. 158, p. 573-607.

Brusatte, S.L., Carr, T.D., and Norell, M.A., 2012, The osteology of Alioramus, a gracile and long-snouted tyrannosaurid (Dinosauria: Theropoda) from the Late Cretaceous of Mongolia: Bulletin of the American Museum of Natural History, v. 366, p. 1-197.

Buckley, L.G., McCrea, R.T., and Lockley, M.G., 2015, Birding by foot: a critical look at the synapomorphy-and phonetic-based approaches to 
trackmaker identification of enigmatic tridactyl Mesozoic traces: Ichnos, v. 22, p. $192-207$.

Burch, S.H., 2014, Complete forelimb myology of the basal theropod dinosaur Tawa hallae based on a novel robust muscle reconstruction method: Journal of Anatomy, v. 225, p. 271-297.

Butler, R.J., Barrett, P.M., and Gower, D.J., 2012, Reassessment of the evidence for postcranial skeletal pneumaticity in Triassic archosaurs, and the early evolution of the avian respiratory system: PLOS One, v. 7, p. e34094.

Camp, C.L., 1936, A new type of small bipedal dinosaur from the Navajo Sandstone of Arizona: Univesity of California Publications, Bulletin of the Department of Geological Sciences, v. 24, p. 39-534.

Carpenter, K.A., 1997, A giant coelophysoid (Ceratosauria) theropod from the Upper Triassic of New Mexico: Neues Jahrbuch für Geologie und Paläontologie-Abhandlungen, v. 205, p. 189-208.

Carr, T.D., 1999, Craniofacial ontogeny in Tyrannosauridae (Dinosauria, Coelurosauria): Journal of Vertebrate Paleontology, v. 19, p. 497-520.

Carrano, M.T., and Hutchinson, J.R., 2002, Pelvic and hindlimb musculature of Tyrannosaurus rex (Dinosauria: Theropoda): Journal of Morphology, v. 253 , p. $207-228$

Carrano, M.T., and Sampson, S.D., 2008, The phylogeny of Ceratosauria (Dinosauria: Theropoda): Journal of Systematic Paleontology, v. 6, p. 183-236.

Carrano, M.T., Sampson, S.D., and Forster, C.A., 2002, The osteology of Masiakasaurus knopfleri, a small abelisauroid (Dinosauria: Theropoda) from the Late Cretaceous of Madagascar: Journal of Vertebrate Paleontology, v. 22, p. $510-534$.

Carrano, M.T., Hutchinson, J.R., and Sampson, S.D., 2005, New information on Segisaurus halli, a small theropod dinosaur from the Early Jurassic of Arizona: Journal of Vertebrate Paleontology, v. 25, p. 835-849.

Carrano, M.T., Benson, R.B.J., and Sampson, S.D., 2012, The phylogeny of Tetanurae (Dinosauria: Theropoda): Journal of Systematic Paleontology, v. 10 , p. $211-300$.

Chapelle, K.E.J., and Choiniere, J.N., 2018, A revised cranial description of Massospondylus carinatus Owen (Dinosauria: Sauropodomorpha) based on computed tomographic scans and a review of cranial characters for basal Sauropodomorpha: PeerJ, v. 6, p. e4224.

Charig, A.J., and Milner, A.C., 1990, The systematic position of Baryonyx walkeri, in the light of Gauthier's reclassification of the Theropoda, in Carpenter, K., and Currie, P.J., eds., Dinosaur Systematics: Approaches and Persepctives: Cambridge, Cambridge University Press, p. 127-145.

Charig, A.J., Attridge, J., and Cromtpon, A.W., 1965, On the origin of the sauropods and the classification of the Saurischia: Proceedings of the Linnean Society of London, v. 176, p. 197-221.

Clark, J.M., Norell, M.A., and Rowe, T.B., 2002, Cranial anatomy of Citipati osmolskae (Theropoda, Oviraptorosauria), and a reinterpretation of the holotype of Oviraptor philoceratops: American Museum Novitates, v. 3364, p. $1-24$.

Coddington, J., and Scharff, N., 1994, Problems with zero-length branches: Cladistics, v. 10 , p. $415-423$.

Colbert, E.H., 1981, A primitive ornithischian dinosaur from the Kayenta Formation of Arizona: Museum of Northern Arizona Bulletin, v. 53, p. 1-61.

Colbert, E.H., 1989, The Triassic dinosaur Coelophysis: Museum of Northern Arizona Bulletin, v. 57, p. 1-160.

Cooley, M.E., Harshbarger, J.W., Akers, J.P., Hardt, W.F., and Nicks, O., 1969, Regional hydrogeology of the Navajo and Hopi indian reservations, Arizona, New Mexico, and Utah: United States Geological Survey Professional Paper, v. 521-A, p. 1-61.

Cooper, M.R., 1981, The prosauropod dinosaur Massospondylus carinatus Owen from Zimbabwe: its biology, mode of life and phylogenetic significance: Occasional Papers of the National Museums and Monuments of Rhodesia, Series B, Natural Sciences, v. 6, p. 689-840.

Cope, E.D., 1869, Synopsis of the extinct Batrachia, Reptilia and Aves of North America: Transactions of the American Philosophical Society, v. 14, p. 1-252.

Cope, E.D., 1887, The dinosaurian genus Coelurus: American Naturalist, v. 21, p. 367-369

Coria, R.A., and Currie, P.J., 2016, A new megaraptoran dinosaur (Dinosauria, Theropoda, Megaraptoridae) from the Late Cretaceous of Patagonia: PLOS One, v. 11, p. e0157973.

Crompton, A.W., and Charig, A.J., 1962, A new ornithischian from the Upper Triassic of South Africa: Nature, v. 196, p. 1074-1077.

Currie, P.J., and Zhao, X.-J., 1993, A new carnosaur (Dinosauria, Theropoda) from the Jurassic of Xinjiang, People's Republic of China: Canadian Journal of Earth Sciences, v. 30, p. 2037-2081.

Dal Sasso, C., Maganuco, S., Buffetaut, E., and Mendez, M.A., 2005, New information on the skull of the enignamtic theropod Spinosaurus, with remarks on its size and affinities: Journal of Vertebrate Paleontology, v. 25, p. 888-896.

Dal Sasso, C., Maganuco, S., and Cau, A., 2018, the oldest ceratosaurian (Dinosauria: Theropoda), from the Lower Jurassic of Italy, sheds light on the evolution of the three-fingered hand of birds: PeerJ, v. 6, p. e5976.
DeBlieux, D.D., Madsen, S.K., Kirkland, J.H., Inkenbrandt, P., and Santucci, V.L., 2012, Significant Mesozoic vertebrate fossil localities discovered during the continuing paleontological resource inventory and monitoring at Arches National Park: Journal of Vertebrate Paleontology, Program and Abstracts, 2012, p. 85

Depéret, C., 1896, Note sur les Dinosauriens Sauropodes et Théropodes du Crétacé supérieur de Madagascar: Bulletin de la Societe Geologique de France, v. 21, p. 176-194.

de Queiroz, K., and Gauthier, J.A., 1992, Phylogenetic taxonomy: Annual Review of Ecology and Systematics, v. 23, p. 449-480.

Ezcurra, M.D., 2006, The cranial anatomy of the coelophysoid theropod Zupaysaurus rougieri from the Upper Triassic of Argentina: Historical Biology, v. 19 , p. $185-202$.

Ezcurra, M.D., 2017, A new early coelophysoid neotheropod from the Late Triassic of northwestern Argentina: Ameghiniana, v. 54, p. 506-538.

Ezcurra, M.D., and Brusatte, S.L., 2011, Taxonomic and phylogenetic reassessment of the early theropod dinosaur Camposaurus arizonensis from the Late Triassic of North America: Palaeontology, v. 54, p. 763-772.

Ezcurra, M.D., and Cuny, G., 2007, The coelophysoid Lophostropheus airlensis, gen. nov.: a review of the systematics of "Liliensternus" airlensis from the Triassic-Jurassic outcrops of Normandy (France): Journal of Vertebrate Paleontology, v. 27, p. 73-86.

Ezcurra, M.D., and Novas, F.E., 2007, Phylogenetic relationships of the Triassic theropod Zupaysaurus rougieri from NW Argentina: Historical Biology, v. 19 , p. $35-72$.

Felsenstein, J., 1985, Confidence limits on phylogenies: an approach using the bootstrap: Evolution, v. 39, p. 783-791.

Forster, C.A., 1999, Gondwanan dinosaur evolution and biogeographic analysis: Journal of African Earth Sciences, v. 28, p. 169-185.

Gates, T.A., Organ, C., and Zanno, L.E., 2016, Bony cranial ornamentation linked to rapid evolution of gigantic theropod dinosaurs: Nature Comunications, v. 7, p. 12931.

Gauthier, J.A., 1986, Saurischian monophyly and the origin of birds: Memoirs of the California Academy of Sciences, v. 8, p. 1-55.

Gay, R., 2001, New specimens of Dilophosaurus wetherilli (Dinosauria: Theropoda) from the Early Jurassic Kayenta Formation of northern Arizona: Mesa Southwest Museum Bulletin, v. 8, p. 19-23.

Gay, R., 2005, Sexual dimorphism in the Early Jurassic theropod dinosaur Dilophosaurus and a comparison with other related forms, in Paul, G.S. ed., The Carnivorous Dinosaurs: Bloomington, Indiana University Press, p. 277-283.

Goloboff, P., Farris, J., and Nixon, K., 2008, TNT: a free program for phylogenetic analysis: Cladistics, v. 24, p. 774-786.

Gower, D.J., and Weber, E., 1998, The braincase of Euparkeria, and the evolutionary relationships of birds and crocodilians: Biological Reviews, v. 73, p. 367-411.

Gill, F.B., Slikas, B., and Sheldon, F.H., 2005, Phylogeny of titmice (Paridae) II. Species relationships based on sequences of the mitochondrial cyctochrome-B gene: Auk, v. 122, p. 121-143.

Griffin, C.T., 2018, Developmental patterns and variation among early theropods: Journal of Anatomy, v. 232, p. 604-640.

Griffin, C.T., 2019, Large neotheropods from the Upper Triassic of North America and the early evolution of large theropod body sizes: Journal of Paleontology, v. 93, p. 1010-1030.

Griffin, C.T., and Nesbitt, S.J., 2019, Does maximum body size of theropods increase across the Triassic-Jurassic boundary? Integrating ontogeny, phylogeny, and body size: The Anatomical Record, v. 303, p. 1158-1169.

Hammer, W.R., and Hickerson, W.J., 1994, A crested theropod dinosaur from Antarctica: Science, v. 264, p. 828-830.

Haines, R.W., 1942, The tetrapod knee joint: Journal of Anatomy, v. 76, p. 270-301.

Harris, J.D., 2006, The axial skeleton of the dinosaur Suuwassea emilieae (Sauropoda: Flagellicaudata) from the Upper Jurassic Morrison Formation of Montana, USA: Palaeontology, v. 49, p. 1091-1121.

Haubold, H., 1986, Archosaur footprints at the terrestrial Triassic-Jurassic transition, in Padian K., ed., The Beginning of the Age of Dinosaurs: Faunal Change Across the Triassic-Jurassic Boundary: Cambridge, Cambridge University Press, p. 189-201.

Hendrickx, C., and Mateus, O., 2014, Torvosaurus gurneyi n. sp., the largest terrestrial predator from Europe, and a proposed terminology of the maxilla anatomy in nonavian theropods. PLOS One, v. 9, p. 388905.

Hendrickx, C., Hartman, S.A., and Mateus, O., 2015, An overview of non-avian theropod discoveries and classification: PalArch's Journal of Vertebrate Paleontology, v. 12, p. 1-73.

Hitchcock, E., 1845, An attempt to name, classify, and describe, the animals that made the fossil footmarks of New England: Annual Meeting of the Association of American Geologists and Naturalists, New Haven Connecticut, Abstracts and Proceedings, v. 6, p. 23-25.

Holtz, T.R., 1994, The phylogenetic position of the Tyrannosauridae: implications for theropod systematics: Journal of Vertebrate Paleontology, v. 68, p. $1100-1117$. 
Holtz, T.R., 2000, A new phylogeny of the carnivorous dinosaurs: Gaia, v. 15, p. 5-61.

Hopson, J.A., 1975, The evaluation of cranial display structures in hadrosaurian dinosaurs: Paleobiology, v. 1, p. 21-43.

Hopson, J.A., 1979, Paleoneurology, in Gans, C., Northcutt, R.G., and Ulinski, P., eds., Biology of the Reptilia, Vol. 9, Neurology A: London, Academic Press, p. 39-146.

Hu, D., Clarke, J.A., Elaison, C.M., Qiu, R., Li, Q., Shawkey, M.D., Zhao, C., D'Alba, L., Jiang, J., and Xu, X., 2018, A bony-crested Jurassic dinosaur with evidence of iridescent plumage highlights complexity in early paravian evolution: Nature Communications, v. 9, p. 217.

Hu, S., 1993, A new Theropoda (Dilophosaurus sinensis sp. nov.) from Yunnan, China: Vertebrata PalAsiatica, v. 31, p. 65-69.

Hunt, A.P., Lucas, S.G., Heckert, A.B., Sullivan, R.M., and Lockley, M.G., 1998, Late Triassic dinosaurs from the western United States: Geobios, v. 31, p. 511-531.

Hutchinson, J.R., 2001, The evolution of pelvic osteology and soft tissues on the line to extant birds (Neornithes): Zoological Journal of the Linnean Society, v. 131, p. $123-168$.

Hutchinson, J.R., and Gatesy, S.M., 2000, Adductors, abductors, and the evolution of archosaur locomotion: Paleobiology, v. 26, p. 734-751.

Irmis, R.B., Nesbitt, S.J., Padian, K., Smith, N.D., Turner, A.H., Woody, D., and Downs, A., 2007, A Late Triassic dinosauromorph assemblage from New Mexico and the rise of dinosaurs: Science, v. 317, p. 358-361.

Ivie, M.A., Slipinksi, S.A., and Wegrzynowicz, P., 2001, Generic homonyms in the Colydiinae (Coleoptera: Zopheridae): Insecta Mundi, v. 15, p. 63-64.

Janensch, W., 1929, Die Wirbelsäule der Gattung Dicraeosaurus: Palaeontographica, v. 2 (supplement 7), p. 37-133.

Jones, I.L., and Hunter, F.M., 1993, Mutual sexual selection in a monogamous seabird: Nature, v 362, p. 238-239.

Jones, I.L., and Hunter, F.M., 1999, Experimental evidence for mutual interand intrasexual selection favouring a crested auklet ornament: Animal Behavior, v. 57, p. 521-528.

Kermack, D.M., 1982, A new tritylodontid from the Kayenta Formatoin of Arizona: Zoological Journal of the Linnean Society, v. 76, p. 1-17.

Knoll, F., Witmer, L.M., Ortega, F., Ridgely, R.C., and Schwarz-Wings, D., 2012, The braincase of the basal sauropod dinosaur Spinophorosaurus and 3D reconstructions of the cranial endocast and inner ear: PLOS One, v. 7, p. e30060.

Lamanna, M.B., Sues, H.-D., Schachner, E.R., and Lyson, T.R., 2014, A new large-bodied oviraptorosaurian theropod dinosaur from the latest Cretaceous of western North America: PLOS One, v. 9, p. e92022.

Langer, M.C., 2003, The pelvic and hind limb anatomy of the stemsauropodomorph Saturnalia tupiniquim (Late Triassic, Brazil) : PaleoBios, v. 23 , p. $1-30$.

Langer M.C., Abdala, F., Richter, M., and Benton, M., 1999, A sauropodomorph dinosaur from the Upper Triassic (Carnian) of southern Brazil: Comptes Rendus de l'Académie des Sciences, v. 329, p. 511-517.

Langer, M.C., França, M.A., and Gabriel, S., 2007, The pectoral girdle and forelimb anatomy of the stem-sauropodomorph Saturnalia tupiniquim (Upper Triassic, Brazil): Special Papers in Palaeontology, v. 77 , p. 113-137.

Langer, M.C., Rincón, A.D., Ramezani, J., Solórzano, A., and Rauhut, O.W.M., 2014, New dinosaur (Theropoda, stem-Averostra) from the earliest Jurassic of the La Quinta Formation, Venezuelan Andes: Royal Society Open Science, v. 1, p. 140184.

Langer, M.C., Ezcurra, M.D., Rauhut, O.W.M., Benton, M.K., Knoll, F., McPhee, B.W., Novas, F.E., Pol, D., and Brusatte, S.L., 2017, Untangling the dinosaur family tree: Nature, v. 551, p. E1-E3.

Lautenschlager, S., and Butler, R.J., 2016, Neural and endocranial anatomy of Triassic phytosaurian reptiles and convergence with fossil and modern crocodylians: PeerJ, v. 4, p. e2251.

Lautenschlager, S., Rayfield, E.J., Altangerel, P., Zanno, L.E., and Witmer, L.M., 2012, The endocranial anatomy of Therizinosauria and its implications for sensory and cognitive function: PLOS One, v. 7, p. e52289.

Lessner, E.J., and Stocker, M.R., 2017, Archosauriform endochranial morphology and osteological evidence for semiaquatic sensory adaptations in phytosaurs: Journal of Anatomy, v. 231, p. 655-664.

Lewis, G.E., Irwin, J.W., and Wilson, R.F., 1961, Age of the Glen Canyon Group (Triassic and Jurassic) on the Colorado Plateau: Geological Society of America Bulletin, v. 72, p. 1437-1440.

Linnaeus, C., 1758, Systema naturae per regna tria naturae, secundum classes, ordines, genera, species, cum characteribus, differentiis, synonymis, locis. Editio decima, reformata [10th revised edition], vol. 1: Holmiae, Laurentii Salvii, $824 \mathrm{p}$.

Lockley, M.G., Gierlinski, G.D., and Lucas, S.G., 2011, Kayentapus revisited: notes on the type material and the importance of this theropod footprint ichnogenus: New Mexico Museum of History and Science Bulletin, v. 53, p. $330-336$
Long, R.A., and Murry, P.A., 1995, Late Triassic (Carnian and Norian) tetrapods from the Southwestern United States: New Mexico Museum of Natural History and Science Bulletin, v. 4, p. 1-254.

Lucas, S.G., Klein, H., Lockley, M.G., Spielmann, J.A., Gierlinski, G.D., Hunt, A.P., and Tanner, L.H., 2006a, Triassic-Jurassic stratigraphic distribution of the theropod footprint ichnogenus Eubrontes: New Mexico Museum of Natural History and Science Bulletin, v. 37, p. 86-93.

Lucas, S.G., Lockley, M.G., Hunt, A.P., and Tanner, L.H., 2006b, Biostratigraphic significance of tetrapod footprints from the Triassic-Jurassic Wingate Sandstone on the Colorado Plateau: New Mexico Museum of Natural History and Science Bulletin, v. 37, p. 109-117.

Lull, R.S., 1904, Fossil footprints of the Jura-Trias of North America: Memoirs of the Boston Society of Natural History, v. 5, p. 461-557.

Maddison, W.P., and Maddison, D.R., 2015, Mesquite: a modular system for evolutionary analysis: Version 3.04, http://mesquiteproject.org.

Madsen, Jr., J.H., 1976, Allosaurus fragilis: a revised osteology: Utah Geological and Mining Survey Bulletin, v. 109, p. 1-163.

Madsen, Jr., J.H., and Welles, S.P., 2000, Ceratosaurus (Dinosauria, Theropoda): a revised osteology: Utah Geological Survey Miscellaneous Publication, v. 00-2, p. 1-80.

Madsen, S.K., Kirkland, J.I., DeBlieux, D.D., Santucci, V.L., Inkenbrandt, P., and Tweet, J.S., 2012, Paleontological resources inventory and monitoring, Arches National Park, Utah: Utah Geological Survey Contract Deliverable. Cooperative Agreement \#H230097080, p. 1-119.

Malafaia, E., Ortega, F., Escaso, F., and Silva, B., 2014, New evidence of Ceratosaurus (Dinosauria: Theropoda) from the Late Jurassic of the Lusitanian Basin, Portugal: Historical Biology, v. 27, 938-946.

Marsh, A.D., and Rowe, T.B., 2018, Anatomy and systematics of the sauropodomorph Sarahsaurus aurifontanalis from the Early Jurassic Kayenta Formation: PLOS One, v. 13, p. e0204007.

Marsh, A.D., Rowe, T., Simonetti, A., Stockli, D., and Stockli, L., 2014, The age of the Kayenta Formation of northeastern Arizona: overcoming the challenges of dating fossil bone: Journal of Vertebrate Paleontology, SVP Program and Abstracts Book 2014, p. 178.

Marsh, A.D., Parker, W.G., Langer, M.C., and Nesbitt, S.J., 2019, Redescription of the holotype specimen of Chindesaurus bryansmalli Long and Murry, 1995 (Dinosauria: Theropoda) from Petrified Forest National Park, Arizona: Journal of Vertebrate Paleontology, v. 39, p. e1645682

Marsh, O.C., 1877, Notice of new dinosaurian reptiles from the Jurassic formation: American Journal of Science, Series 3, v. 14, p. 514-516.

Marsh, O.C., 1881, Principal characters of American Jurassic dinosaurs, part V: American Journal of Science, Series 3, v. 21, p. 417-423.

Marsh, O.C., 1884, Principle characters of American Jurassic dinosaurs, part VIII: the order Theropoda: American Journal of Science, Series 3, v. 27, p. 329-340.

Martill, D.M., Vidovic, S.U., Howells, C., and Nudds, J.R., 2016, The oldest Jurassic dinosaur: a basal neotheropod from the Hettangian of Great Britain: PLOS One, v. 11, p. e0145713.

Martínez, R.N., Sereno, P.C., Alcobar, O.A., Colombi, C.E., Renne, P.R., Montañez, I.P., and Currie, B.S., 2011, A basal dinosaur from the dawn of the dinosaur era in southwestern Pangaea: Science, v. 331, p. 206-210.

Marzoli, A., Renne, P.R., Piccirillo, E.M., Ernesto, M., Bellieni, G., and De Min, A., 1999, Extensive 200-million-year-old continental flood basalts of the Central Atlantic Magmatic Province: Science, v. 284, p. 616-618.

McPhee, B.W., Yates, A.M., Choiniere, J.H., and Abdala, F., 2014, The complete anatomy and phylogenetic relationships of Antetonitrus ingenipes (Sauropodiformes, Dinosauria): implications for the origins of Sauropoda: Zoological Journal of the Linnean Society, v. 171, p. 151-205.

Milner, A.R.C., Lockley, M.G., and Kirkland, J.I., 2006, Large collection of well-preserved theropod dinosaur swim traces from the Lower Jurassic Moenave Formation, St. George, Utah: New Mexico Museum of Natural History and Science Bulletin, v. 37, p. 315-328.

Milner, A.R.C., Harris, J.D., Lockley, M.G., Kirkland, J.I., and Matthews, N.A., 2009, Bird-like anatomy, posture, and behavior revealed by an Early Jurassic theropod dinosaur resting trace: PLOS One, v. 4, p. e4591.

Molnar, R.E., 1977, Analogies in the evolution of combat and display structures in ornithopods and ungulates: Evolutionary Theory, v. 3, p. $165-190$.

Moser, M., 2004, Plateosaurus engelhardti Meyer, 1837 (Dinosauria: Sauropodomorpha) aus dem Feuerletten (Mittelkeuper; Obertrias) von Bayern: Zitteliana Reihe B, Abhandlungen der Bayerischen Staatssammlung für Paläontologie und Geologie, v. 24, p. 1-186.

Nesbitt, S.J., 2011, The early evolution of archosaurs: relationships and the origin of major clades: Bulletin of the American Museum of Natural History, v. 352 , p. $1-292$.

Nesbitt, S.J., and Ezcurra, M.D., 2015, The early fossil record of dinosaurs in North America: a new neotheropod from the base of the Upper Triassic Dockum Group of Texas: Acta Palaeontologica Polonica, v. 60, p. 513-526. 
Nesbitt, S.J., and Stocker, M.R., 2008, The vertebrate assemblage of the Late Triassic Canjilon Quarry (Northern New Mexico, USA) and the importance of apomorphy-based assemblage comparisons: Journal of Vertebrate Paleontology, p. 28, p. 1063-1072.

Nesbitt, S.J., Irmis, R.B., Parker, W.G., Smith, N.D., Turner, A.H., and Rowe, T., 2009a, Hindlimb osteology and distribution of basal dinosauromorphs from the Late Triassic of North America: Journal of Vertebrate Paleontology, v. 29 , p. $498-516$.

Nesbitt, S.J., Smith, N.D., Irmis, R.B., Turner, A.H., Downs, A., and Norell, M.A., 2009b, A complete skeleton of a Late Triassic saurischian and the early evolution of dinosaurs: Science, v. 326, p. 1530-1533.

Nopsca, B.F., 1928, The genera of reptiles: Palaeobiologica, v. 1, p. 163-188.

Novas, F.E., 1994, New information on the systematics and postcranial skeleton of Herrerasaurus ischigualastensis (Theropoda: Herrerasauridae) from the Ischigualasto Formation (Upper Triassic) of Argentina: Journal of Vertebrate Paleontology, v. 13, p. 400-432.

Novas, F.E., Suárez, S.L., Angolín, F.L., Ezcurra, M.N., Chimento, N.S.R., de la Cruz, R., Isasi, M.P., Vargas, A.O., and Rubilar-Rogers, D., 2015, An enigmatic plant-eating theropod from the Late Jurassic period of Chile: Nature, v. 522, p. 331-334.

O'Connor, P.M., 2006, Postcranial pneumaticity: an evaluation of soft-tissue influences on the postcranial skeleton and the reconstruction of pulmonary anatomy in archosaurs: Journal of Morphology, v. 267, p. 1199-1226.

Olsen, P.E., Smith, J.B., and McDonald, N.G., 1997, Type material of the type species of the classic theropod footprint genera Eubrontes, Anchisauripus, and Grallator (Early Jurassic, Hartford and Deerfield basins, Connecticu and Massachusetts, U.S.A.): Journal of Vertebrate Paleontology, v. 18, p. 586-601.

Osborn, H.F., 1899, A skeleton of Diplodocus: Memoirs of the American Museum of Natural History, v. 1, p. 191-214.

Osborn, H.F., 1905, Tyrannosaurus and other Cretaceous carnivorous dinosaurs: American Musuem of Natural History Bulletin, v. 21, p. 259-265.

Osborn, H.F., 1924, Three new Theropoda, Protoceratops zone, central Mongolia: American Museum Novitates, v. 144, p. 1-12.

Osborn, H.F., and Mook, C.C., 1921, Camarasaurus, Amphocoelias, and other sauropods of Cope: Memoirs of the American Museum of Natural History, v. 3 , p. $247-387$.

Ostrom, J.H., 1978, The osteology of Compsognathus longipes Wagner: Zitteliana, v. 4, p. 730118.

Owen, R., 1842, Report on British fossil reptiles, part II: Report of the British Association of the Advancement of Science, v. 11, p. 60-104.

Owen, R., 1854, Descriptive catalogue of the fossil organic remains of Reptilia and Pisces contained in the Museum of the Royal College of Surgeons of England: London, Taylor and Francis, $184 \mathrm{p}$.

Owen, R., 1861, A monograph of a fossil dinosaur (Scelidosaurus harrisonii, Owen) of the Lower Lias, part I: Monographs on the British Fossil Reptilia from the Oolitic Formations, v. 1, p. 1-14.

Padian, K., 1986, On the type material of Coelophysis Cope (Saurischia: Theropoda) and a new specimen from the Petrified Forest of Arizona (Late Triassic: Chinle Formation), in Padian, K., ed., The Beginning of the Age of Dinosaurs: Faunal Change Across the Triassic-Jurassic Boundary: Cambridge, Cambridge University Press, p. 203-221.

Padian, K., 1989, Presence of the dinosaur Scelidosaurus indicates Jurassic age for the Kayenta Formation (Glen Canyon Group, northern Arizona): Geology, v. 17, p. 438-441.

Padian, K., and Horner, J.R., 2014, The species recognition hypothesis explains exaggerated structures in non-avialan dinosaurs better than sexual selection does: Comptes Rendus Paleovol, v. 13, p. 97-107.

Parker, W.G., 2008, Description of new material of the aetosaur Desmatosuchus spurensis (Archosauria: Suchia) from the Chinle Formation of Arizona and a revision of the genus Desmatosuchus: PaleoBios, v. 28, p. 1-40.

Parker, W.G., 2018, Redescription of Calyptosuchus (Stagonolepis) wellesi (Archosauria: Pseudosuchia: Aetosauria) from the Late Triassic of the southwestern United States with a discussion of genera in vertebrate paleontology: PeerJ, v. 6, p. e4291.

Paul, G.S., 1988, Predatory Dinosaurs of the World: A Complete Illustrated Guide: New York, Simon and Schuster, $464 \mathrm{p}$

Paul, G.S., 2002, Dinosaurs of the Air: The Evolution and Loss of Flight in Dinosaurs and Birds: Baltimore, Johns Hopkins University Press, 472 p.

Paulina-Carabajal, A., and Currie, P.J., 2017, The braincase of the theropod dinosaur Murusraptor: osteology, neuroanatomy and comments on the paleobiological implications of certain endocranial features: Ameghiniana, v. 54, p. 617-640.

Paulina-Carabajal, A., Ezcurra, M.D., and Novas, F.E., 2019, new information on the braincase and endocranial morphology of the Late Triassic neotheropod Zupaysaurus rougieri using computed tomography data: Journal of Vertebrate Paleontology, v. 39, p. e1630421.
Pearsons IV, W.S., and Currie, P.J., 2011, Dinosaur speed demon: the caudal musculature of Carnotaurus sastrei and implications for the evolution of South American abelisaurids: PLOS One, v. 6, p. e25763.

Piechowski, R., Niedźwiedzki, G., and Tałanda, M., 2019, Unexpected bird-like features and high intraspecific variation in the braincase of the Triassic relative of dinosaurs: Historical Biology, v. 31, p. 1065-1081.

Prieto-Márquez, A., and Norell, M.A., 2011, Redescription of a nearly complete skull of Plateosaurus (Dinosauria: Sauropodomorpha) from the Late Triassic of Trissingen (Germany): American Museum Novitates, v. 2327, p. 1-59.

Raath, M.A., 1969, A new coelurosaurian dinosaur from the Forest Sandstone of Rhodesia: Arnoldia Rhodesia, v. 4, p. 1-25.

Raath, M.A., 1977, The anatomy of the Triassic theropod Syntarsus rhodesiensis (Saurischia: Podekesauridae) and a consideration of its biology [Ph.D. dissertation]: Grahamstown, South Africa, Rhodes University, 233 p.

Rauhut, O.W.M., 2003, The interrelationships and evolution of basal theropod dinosaurs: Special Papers in Palaeontology, v. 69, p. 1-213.

Regnault, S., Pitsillides, A.A., and Hutchinson, J.R., 2014, Structure, ontogeny and evolution of the patellar tendon in emus (Dromaius novaehollandiae) and other palaeognath birds: PeerJ, v. 2, p. e711.

Regnault, S., Jones, M.E.H., Pitsillides, A.A., and Hutchinson, J.R., 2016, Anatomy, morphology and evolution of the patella in squamate lizards and tuatara (Sphenodon punctatus): Journal of Anatomy, v. 228, p. 864-876.

Reig, O.A., 1963, La presencia de dinosaurios saurisquios en los "Estrados de Ischigualasto" (Mesotriásico Superior) de las provincias de San Juan y La Rioja (República Argentina): Ameghiniana, v. 3, p. 3-20.

Rogers, S.W., 1999, Exploring dinosaur neuropaleobiology: computed tomography scanning and analysis of an Allosaurus fragilis endocast: Neuron, v. 21 , p. $162-173$.

Rowe, T.B., 1989, A new species of the theropod dinosaur Syntarsus from the Early Jurassic Kayenta Formation of Arizona: Journal of Vertebrate Paleontology, v. 9, p. 12-136.

Rowe, T.B., and Gauthier, J., 1990, Ceratosauria, in Weishampel, D.B., Dodson, P., and Osmólska, H., eds., The Dinosauria (1st edition): Berkeley, University of California Press, p. 151-168.

Rowe, T.B., Sues, H.-D., and Reisz, R.R., 2011, Dispersal and diversity in the earliest North American sauropodomorph dinosaurs, with a description of a new taxon: Proceedings of the Royal Society of London B: Biological Sciences, v. 278, p. 1044-1053.

Sampson, S.D., and Witmer, L.M., 2007, Craniofacial anatomy of Majungasaurus crenatissimus (Theropoda: Abelisauridae) from the Late Cretaceous of Madagascar: Journal of Vertebrate Paleontology, v. 27 (Memoir 8), p. 32-102.

Samuels, M.S., Regnault, S., and Hutchinson, J.R., 2017, Evolution of the patellar sesamoid bone in mammals: PeerJ, v. 5, p. e3103.

Sanders, K., and Smith, D.K., 2005, The endocranium of the theropod dinosaur Ceratosaurus studied with computed tomography: Acta Paleontologica Polonica, p. 50, p. 601-616.

Schachner, E.R., Manning, P.L., and Dodson, P., 2011, Pelvic and hindlimb myology of the basal archosaur Poposaurus gracilis (Archosauria: Poposauroidea): Journal of Morphology, v. 272, p. 1464-1491.

Schaltegger, U., Guex, J., Bartolini, A., Schoene, B., and Ovtcharova, M., 2008, Precise U-Pb age constraints for end-Triassic mass extinction, its correlation to volcanism and Hettangian post-extinction recovery: Earth and Planetary Science Letters, v. 267, p. 266-275.

Sciscio, L., Bordy, E.M., Abrahams, M., Knoll, F., and McPhee, B.W., 2017 The first megatheropod tracks from the Lower Jurassic upper Elliot Formation, Karoo Basin, Lesotho: PLOS One, v. 12, p. e0185941.

Seeley, H.G., 1888, On the classification of the fossil animals commonly named Dinosauria: Proceedings of the Royal Society of London, v. 43, p. 165-171.

Senter, P., and Juengst, S.L., 2016, Record-breaking pain: the largest number and variety of forelimb bone maladies in a theropod dinosaur: PLOS One, v. 11, p. e0149140.

Senter, P., and Sullivan, C.R., 2019, Forelimbs of the theropod dinosaur Dilophosaurus wetherilli: range of motion, influence of paleopathology and soft tissues, and description of a distal carpal bone: Palaeontologia Electronica, v. 22, p. 1-9.

Sereno, P.C., 1991, Lesothosaurus, "Fabrosaurids," and the early evolution of Ornithischia: Journal of Vertebrate Paleontology, v. 11, p. 168-197.

Sereno, P.C., 2005, The logical basis of phylogenetic taxonomy: Systematic Biology, v. 54, p. 595-619.

Sereno, P.C., 2012, Taxonomy, morphology, masticatory function and phylogeny of heterodontosaurid dinosaurs: ZooKeys, v. 226, p. 1-225.

Sereno, P.C., and Novas, F.E., 1994, The skull and neck of the basal theropod Herrerasaurus ischigualastensis: Journal of Vertebrate Paleontology, v. 13, p. 451-476.

Sereno, P.C., Forster, C.A., Rogers, R.R., and Moneta, A.M., 1993, Primitive dinosaur skeleton from Argentina and the early evolution of the Dinosauria: Nature, v. 361, p. 64-66. 
Sereno, P.C., Martínez, R.N., and Alcobar, O.A., 2012, Osteology of Eoraptor lunensis (Dinosauria, Sauropodomorpha): Journal of Vertebrate Paleontology, v. 32 (Memoir 12), p. 83-179.

Smith, N.D., Makovicky, P.J., Hammer, W.R., and Currie, P.J., 2007, Osteology of Cryolophosaurus ellioti (Dinosauria: Theropoda) from the Early Jurassic of Antarctica and implications for early theropod evolution: Zoological Journal of the Linnean Society, v. 151, p. 377-421.

Sobral, G., Sookias, R.B., Bhullar, B.-A.S., Smith, R., Butler, R.J., and Müller, J., 2016, New information on the braincase and inner ear of Euparkeria capensis Broom: implications for diapsid and archosaur evolution: Royal Society Open Science, v. 3, p. 160072.

Sookias, R.B., Butler, R.J., and Benson, R.B.J., 2012, Rise of dinosaurs reveals major body-size transitions are driven by passive processes of trait evolution: Proceedings of the Royal Society of London B: Biological Sciences, v. 279 , p. $2180-2187$.

Steel, R., 1970, Die Dinosaurier: Wittenberg, Germany, Ziemsen, 95 p.

Stromer, E., 1915, Ergebnisse der Forschungsreisen Professor E. Stromer in den Wüsten Ägyptens, II, Wirbeltier-Reste der Baharije-Stufe (unterested Cenoman), 3, Das Original des Theropoden Spinosaurus aegyptiacus nov. gen., nov. spec.: Abhandlungen der Königlich Bayerischen Akademie der Wissenschaften, Mathematische-Physikalische Klasse, v. 28, p. 1-32.

Stuessy, T.F., 2009, Plant Taxonomy: The Systematic Evaluation of Comparative Data: New York, Columbia University Press, 568 p.

Sues, H.-D., Clark, J.M., and Jenkins, Jr., F.A., 1994, A review of the Early Jurassic tetrapods from the Glen Canyon Group of the American Southwest, in Fraser, N.C., and Sues, H.-D., eds., In the Shadow of the Dinosaurs: Early Mesozoic Tetrapods: Cambridge, Cambridge University Press, p. 284-294.

Sues, H.-D., Reisz, R.R., Hinic, S., and Raath, M.A., 2004, On the skull of Massospondylus carinatus Owen, 1854 (Dinosauria: Sauropodomorpha) from the Eliot and Clarens formations (Lower Jurassic) of South Africa: Annals of the Carnegie Museum, v. 73, p. 239-257.

Sues, H.-D., Nesbitt, S.J., Berman, D.S., and Henrici, A.C., 2011, A latesurviving basal theropod dinosaur from the latest Triassic of North America: Proceedings of the Royal Society of London B: Biological Sciences, v. 278, p. 3459-3464.

Swinton, W.E., 1955, Megalosaurus, the Oxford dinosaur: Advances in Science, v. 12, p. 130-134.

Tykoski, R.S., 1998, The osteology of Syntarsus kayentakatae and its implications for ceratosaurid phylogeny [M.Sc. thesis]: Austin, The University of Texas at Austin, $217 \mathrm{p}$.

Tykoski, R.S., 2005, Anatomy, ontogeny, and phylogeny of coelophysoid theropods [Ph.D. dissertation]: Austin, The University of Texas at Austin, 553 p.

Tykoski, R.S., and Rowe, T.B., 2004, Ceratosauria, in Weishampel, B., Dodson, P., and Osmólska, H., eds., The Dinosauria (2nd edition): Berkeley, University of California Press, p. 47-70.

Tykoski, R.S., Forster, C.A., Rowe, T.B., Sampson, S.D., and Munyikwa, D., 2002, A furcula in the coelophysoid theropod Syntarsus: Journal of Vertebrate Paleontology, v. 22, p. 728-733.

von Huene, F.D., 1932, Die Reptil-Ordnung Saurischia, ihre Entwicklung und Gaschichte: Monographien zur Geologie und Paläontologie, v. 1, p. 1-361.

von Huene, F.D., 1934, Ein neuer Coelurosaurier in der thüringischen Trias: Paläontologische Zeitschrift, v. 16, p. 145-170.

von Meyer, H., 1837, Briefliche Mitteilung an Professor Bronn über Plateosaurus engelhardti: Neues Jahrbuch für Mineralogie, Geognosie, Geologogie und Petrefaktenkunde, 1837, p. 314-316.

von Meyer, H., 1861, Vogel-Federn und Palpipes priscus von Solenhofen: Neues Jahrbuch für Mineralogie, Geognosie, Geologie und Petrefaktenkunde, 1861, p. 561.

Walsh, S.A., Barrett, P.M., Milner, A.C., Manley, G., and Witmer, L.M., 2009, Inner ear anatomy is a proxy for deducing auditory capability and behavior in reptiles and birds: Proceedings of the Royal Society of London B: Biological Sciences, v. 276, p. 1355-1360.

Wang, G.-F., You, H.-L., Pan, S.-G., and Wang, T., 2017, A new crested theropod dinosaur from the Early Jurassic of Yunnan Province, China: Vertebrata PalAsiatica, v. 55, p. 177-186.

Wedel, M.J., 2006, Origin of postcranial pneumaticity in dinosaurs: Integrative Zoology, v. 2, p. 80-85.

Welles, S.P., 1954, New Jurassic dinosaur from the Kayenta Formation of Arizona: Geological Society of America Bulletin, v. 65, p. 591-598.
Welles, S.P., 1970, Dilophosaurus (Reptilia, Saurischia), a new name for a dinosaur: Journal of Paleontology, v. 44, p. 989.

Welles, S.P., 1971, Dinosaur footprints from the Kayenta Formation of northern Arizona: Plateau, v. 44, p. 27-38.

Welles, S.P., 1984, Dilophosaurus wetherilli (Dinosauria, Theropoda): osteology and comparisons: Palaeontographica Abteilung A, v. 185, p. 84-180.

Wilson, J.A., 1999, A nomenclature for vertebral laminae in sauropods and other saurischian dinosaurs: Journal of Vertebrate Paleontology, v. 19, p. 639-653.

Wilson, J.A., 2012, New vertebral laminae and patterns of serial variation in vertebral laminae of sauropod dinosaurs: Contributions from the University of Michigan Museum of Paleontology, v. 32, p. 91-110.

Wilson, J.A., D'Emic, M.D., Ikejiri, T., Moacdieh, E.M., and Whitlock, J.A., 2011, A nomenclature for vertebral fossae in sauropods and other saurischian dinosaurs: PLOS One, v. 6, p. e17114.

Wilson, J.P., Woodruff, D.C., Gardner, J.D., Flora, H.M., Horner, J.R., and Organ, C.L., 2016, Vertebral adaptations to large body size in theropod dinosaurs: PLOS One, p. 11, v. e0158962.

Witmer, L.M., 1997, Craniofacial air sinus systems, in Currie, P.J., and Padian, K., eds., Encyclopedia of Dinosaurs: New York, Academic Press, p. 151-159.

Xing, L.-D., 2012, Sinosaurus from southwestern China [M.Sc. thesis]: Edmonton, The University of Alberta, $267 \mathrm{p}$.

Xing, L.-D., Paulina-Carabajal, A., Currie, P.J., Xu, X., Zhang, J., Wang, T., Burns, M.E., and Dong, Z., 2014, Braincase anatomy of the basal theropod Sinosaurus from the Early Jurassic of China: Acta Geologica Sinica, v. 88, p. $1653-1664$.

Xing, L.-D., Wang, Y., Snively, E., Zhang, J., Dong, Z., Burns, M.E., and Currie, P.J., 2015. Model-based identification of mechanical characteristics of Sinosaurus (Theropoda) crests: Acta Geologica Sinica, v. 89, p. 1-11.

Xing, L.-D., Lockley, M.G., Klein, H., Zhang, J., and Persons, IV, W.S., 2016, A new ornithischian-dominated and theropod footprint assemblage from the Lower Jurassic Lufeng Formation of Yunnan Province, China: New Mexico Museum of Natural History and Science Bulletin, v. 74, p. 331-338.

Xing, L.-D., Lockley, M.G., Zhang, J.-P., Klein, H., Kümmell, S.B., Persons, IV, W.S., and Kuang, H.-W., 2017, Theropod tracks from the Lower Jurassic of Gulin area, Sichuan Province, China: Palaeoworld, v. 26, p. $115-123$.

Xu, X., Clark, J.M., Mo, J., Choiniere, J., Forster, C.A., Erickson, G.M., Hone, D.W.E., Sullivan, C., Eberth, D.A., Nesbitt, S.J., Zhao, Q., Hernandez, R., Jia, C.-K., Han, F.-L., and Guo, Y., 2009, A Jurassic ceratosaur from China helps clarify avian digital homologies: Nature, v. 459, p. 940-944.

Yates, A.M., 2005, A new theropod dinosaur from the Early Jurassic of South Africa and its implications for the early evolution of theropods: Palaeontologia Africana, v. 41, p. 105-122.

Yates, A.M., and Kitching, J.W., 2003, The earliest known sauropod dinosaur and the first steps towards sauropod locomotion: Proceedings of the Royal Society of London B: Biological Sciences, v. 270, p. 1753-1758.

Yates, A.M., Bonnan, M.F., Neveling, J., Chinsamy, A., and Blackbeard, M.G., 2009, A new transitional sauropodomorph dinosaur from the Early Jurassic of South Africa and the evolution of sauropod feeding and quadrupedalism: Proceedings of the Royal Society of London B: Biological Sciences, v. 277, p. 787-794.

Yates, A.M., Wedel, M.J., and Bonnan, M.F., 2011, The early evolution of postcranial skeletal pneumaticity in sauropodomorph dinosaurs: Acta Paleontologica Polonica, v. 57, p. 85-100.

You, H.-L., Azuma, Y., Wang, T., Wang, Y.-M., and Dong, Z.-M., 2014, The first well-preserved coelophysoid theropod dinosaur from Asia: Zootaxa, v. 3873 , p. $233-249$.

Young, C.-C., 1940, Preliminary notes on the Lufeng vertebrate fossils: Acta Geologica Sinica, v. 20, p. 235-240.

Zahner, M., and Brinkmann, W., 2019, A Triassic averostran-line theropod from Switzerland and the early evolution of dinosaurs: Nature Ecology and Evolution, v. 3, p. 1146-1152.

Zhao, X.-J., and Currie, P.J., 1993, A large crested theropod from the Jurassic of Xinjiang, People's Republic of China: Canadian Journal of Earth Sciences, v. 30, p. $2027-2036$.

Accepted: 12 March 2020 